\title{
Lanthanide-Catalyzed Tandem Addition of Amines to Cyanoalkenes: Synthesis of Cyclic Amidines
}

\author{
Jinsong Hou ${ }^{a, b}$, Gaosheng Yang ${ }^{a, *}$ and Zhuo Chai ${ }^{a}$
}

${ }^{a}$ MOE Key Laboratory of Functional Molecular Solids, Anhui Laboratory of Molecule-Based Materials, Institute of Organic Chemistry, College of Chemistry and Materials Science, Anhui Normal University, 189 South Jiuhua Road, Wuhu, Anhui 241002, China

${ }^{b}$ College of Material and Chemical Engineering, Chuzhou University, Chuzhou, Anhui 239000, China

\section{Supporting Information}

\section{Content}

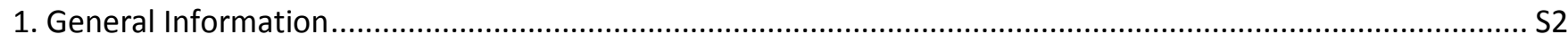

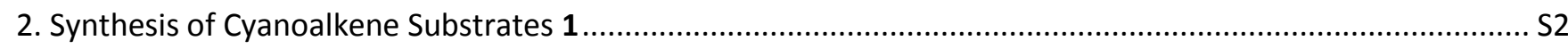

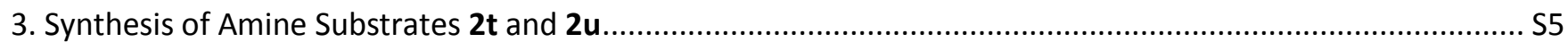

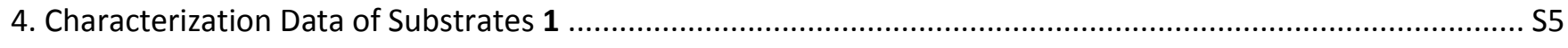

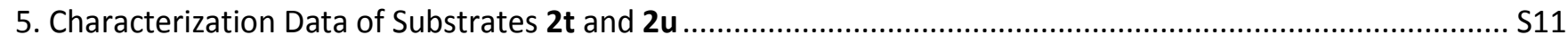

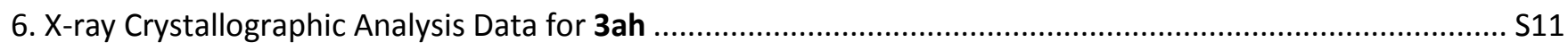

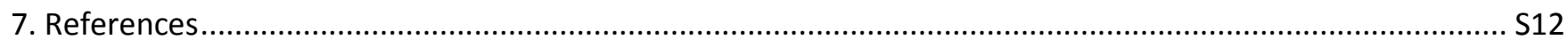

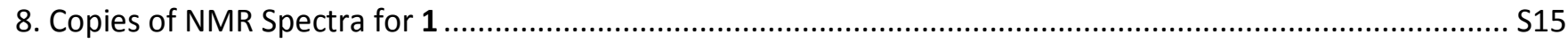

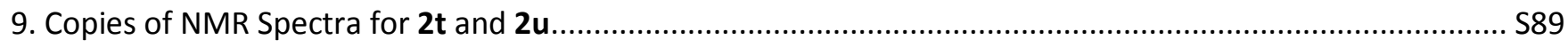

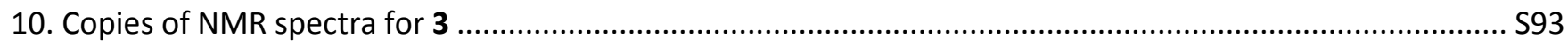

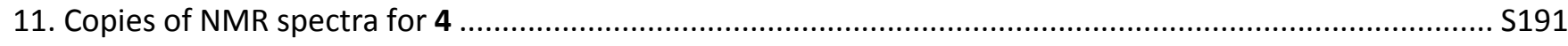

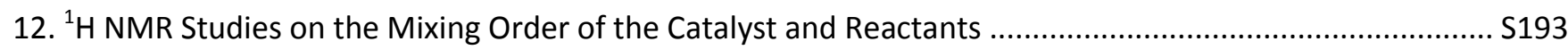

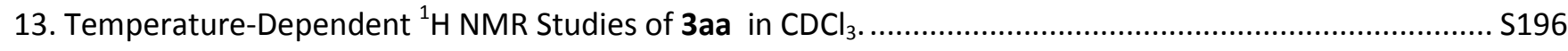




\section{General Information}

All reactions with air- or moisture-sensitive materials were performed in an oven- $\left(120^{\circ} \mathrm{C}\right)$ or flamedried glassware under an inert atmosphere of argon, employing standard Schlenk and glovebox techniques. All solvents were distilled over either finely divided $\mathrm{LiAlH}_{4}$ or sodium benzophenone ketyl under argon prior to use unless otherwise noted. $\mathrm{CDCl}_{3}$ was dried over activated $4 \AA$ molecular sieves. $\mathrm{C}_{6} \mathrm{D}_{6}$ was distilled from sodium/benzophenone ketyl. Flash column chromatography was performed using silica gel (200-400 mesh). For thin-layer chromatography (TLC), silica gel plates (HSGF 254) were used and compounds were visualized by irradiation with UV light. ${ }^{1} \mathrm{H} N M R(500 \mathrm{MHz})$ and ${ }^{13} \mathrm{C}$ NMR $(125 \mathrm{MHz})$ spectra were recorded in $\mathrm{CDCl}_{3}$ or $\mathrm{C}_{6} \mathrm{D}_{6}$. All chemical shifts $(\delta)$ are given in ppm relative to TMS $(\delta=0 \mathrm{ppm})$ as internal standard. Data are reported as follows: chemical shift, multiplicity, coupling constants and integration. High resolution mass spectral (HRMS) data were obtained with an ionization mode of ESI and a TOF analyzer.

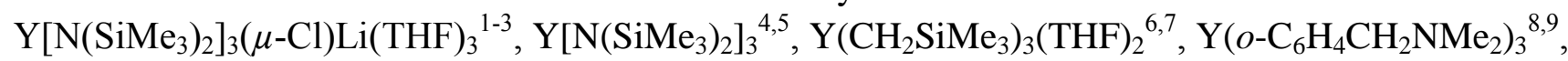
and $\mathrm{RE}\left(\mathrm{CH}_{2} \mathrm{C}_{6} \mathrm{H}_{4} \mathrm{NMe}_{2}-o\right)_{3}{ }^{9,10}$ ( $\left.\mathrm{RE}=\mathrm{Y}, \mathrm{Sc}, \mathrm{Lu}, \mathrm{Sm}, \mathrm{La}\right)$ were prepared by literature methods.

The cyanoalkene substrates pent-4-enenitrile (1zf) and hex-5-enenitrile (1zh) were commercially available.

The amine substrates $N$-methyl-1-phenylmethanamine (2a), $N$-methyl-1-(p-tolyl)methanamine (2b), 1-(4-chlorophenyl)- $N$-methylmethanamine (2c), 1-(4-methoxyphenyl)- $N$-methylmethanamine (2d), dibenzylamine (2e), 1,2,3,4-tetrahydroisoquinoline (2f), diethylamine (2g), pyrrolidine (2h), piperidine (2i), 4-methylpiperidine (2j), 4-chloropiperidine (2k), 4-methoxypiperidine (2l), N,N-dimethylpiperidin4-amine (2m), morpholine (2n), thiomorpholine (2o), 1-methylpiperazine (2p), N-benzyl-1phenylethan-1-amine (2q), $N$-methylaniline (2r) and phenylmethanamine (2s) were commercially available.

\section{Synthesis of Cyanoalkene Substrates 1}

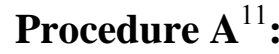

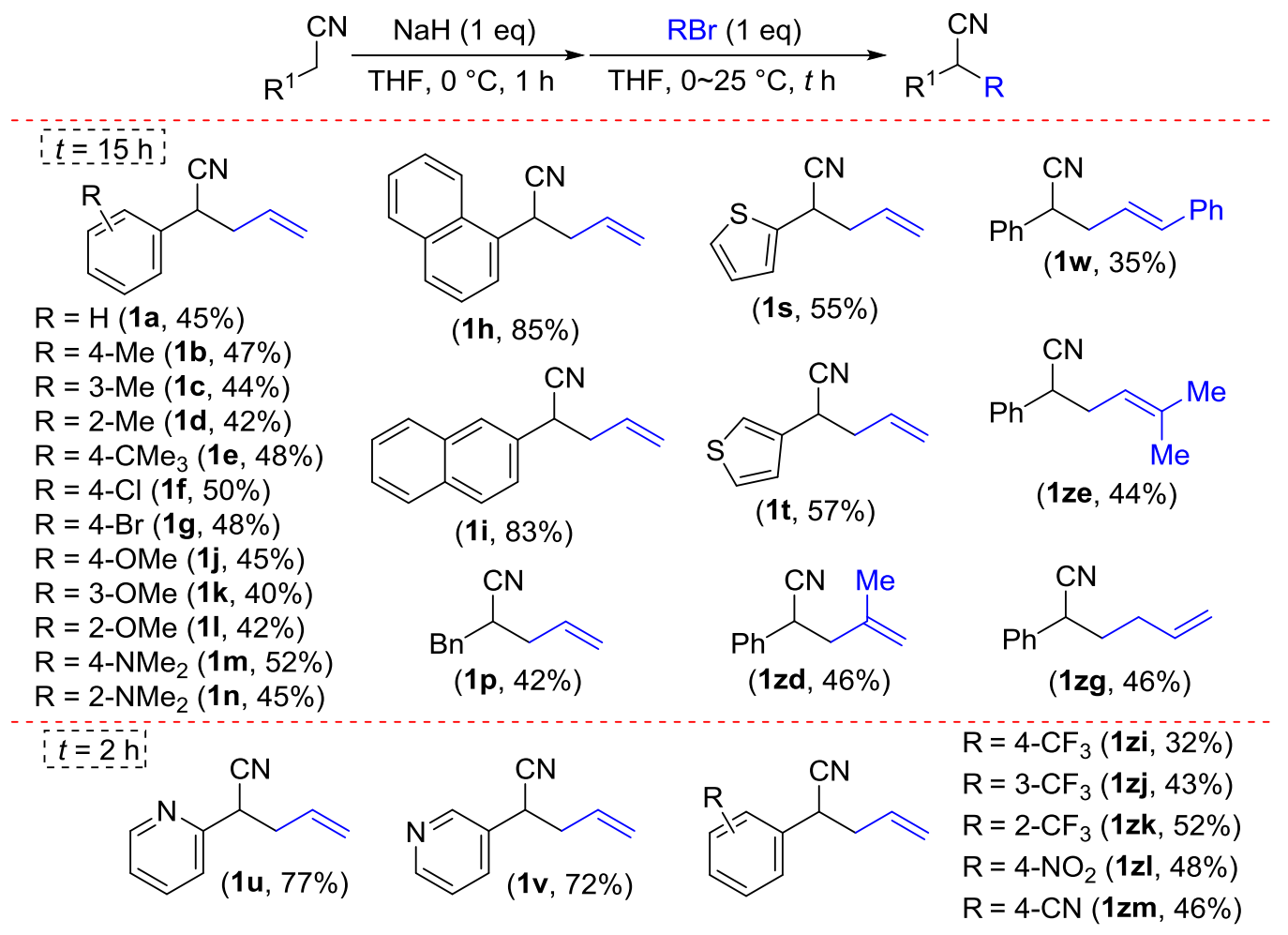


Under an atmosphere of argon, a solution of nitrile $(30.00 \mathrm{mmol})$ in dry THF $(10 \mathrm{~mL})$ was added slowly to a suspension of $\mathrm{NaH}(1.20 \mathrm{~g}, 30.00 \mathrm{mmol}, 60 \%$ dispersion in oil $)$ in dry $\mathrm{THF}(30 \mathrm{~mL})$ at $0{ }^{\circ} \mathrm{C}$. The resulting mixture was stirred for one hour before a solution of alkyl bromide (30.00 mmol) in dry THF $(20 \mathrm{~mL})$ was added dropwise at $0{ }^{\circ} \mathrm{C}$ and the stirring was continued for another one hour. Then the reaction mixture was warmed to room temperature and stirred for 15 hours (or 2 hours). The resulting solution was poured into cold water $(100 \mathrm{~mL})$ and extracted with diethyl ether $(3 \times 60 \mathrm{~mL})$. The combined organic extracts were washed with brine $(2 \times 50 \mathrm{~mL})$, dried over anhydrous $\mathrm{Na}_{2} \mathrm{SO}_{4}$, and filtered. The filtrate was concentrated in vacuo and the residue was purified by column chromatography (silica gel, with the mixture of hexane/ethyl acetate as eluent) to afford $1 \mathbf{a} \sim 1 \mathbf{1 n}, \mathbf{1 p}, \mathbf{1 s}, \mathbf{1 t}, \mathbf{1 u} \sim \mathbf{1 w}, \mathbf{1 z d}$, 1ze, 1zg and 1zi $\mathbf{1 z m}$. The product $\mathbf{1 w}$ was then further purified by recrystallization from petroleum ether/ethyl acetate.

\section{Procedure $\mathbf{B}^{12}$ :}

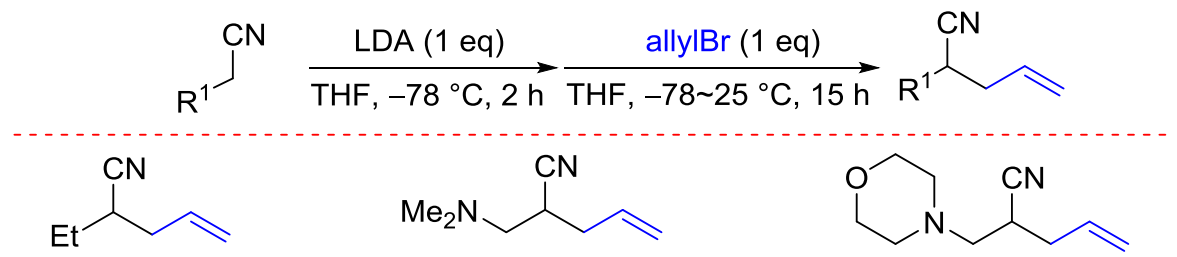

(10, 38\%)

(1q, $40 \%$ )

(1r, 45\%)

To a solution of nitrile $(30.00 \mathrm{mmol})$ in dry THF $(60 \mathrm{~mL})$ was added slowly LDA $(19 \mathrm{~mL}, 30.00 \mathrm{mmol}$, 1.6 $\mathrm{M}$ in THF) at $-78{ }^{\circ} \mathrm{C}$ under an atmosphere of argon. The resulting mixture was stirred for 2 hours before a solution of allyl bromide $(30.00 \mathrm{mmol})$ in dry THF $(20 \mathrm{~mL})$ was added dropwise at $-78{ }^{\circ} \mathrm{C}$. After the reaction mixture was allowed to warm to room temperature to stir for 15 hours, the resulting solution was poured into cold water $(100 \mathrm{~mL})$ and extracted with diethyl ether $(3 \times 60 \mathrm{~mL})$. The combined organic extracts were washed with brine $(2 \times 50 \mathrm{~mL})$, dried over anhydrous $\mathrm{Na}_{2} \mathrm{SO}_{4}$, filtered. The filtrate was concentrated in vacuo and the residue was purified by vacuum distillation to afford 1o, $\mathbf{1 q}$ and $\mathbf{1 r}$.

Procedure $\mathbf{C}^{11,13}$ :

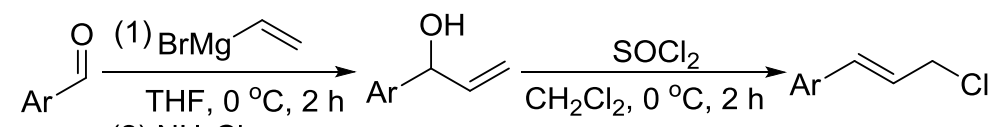
(2) $\mathrm{NH}_{4} \mathrm{Cl}$

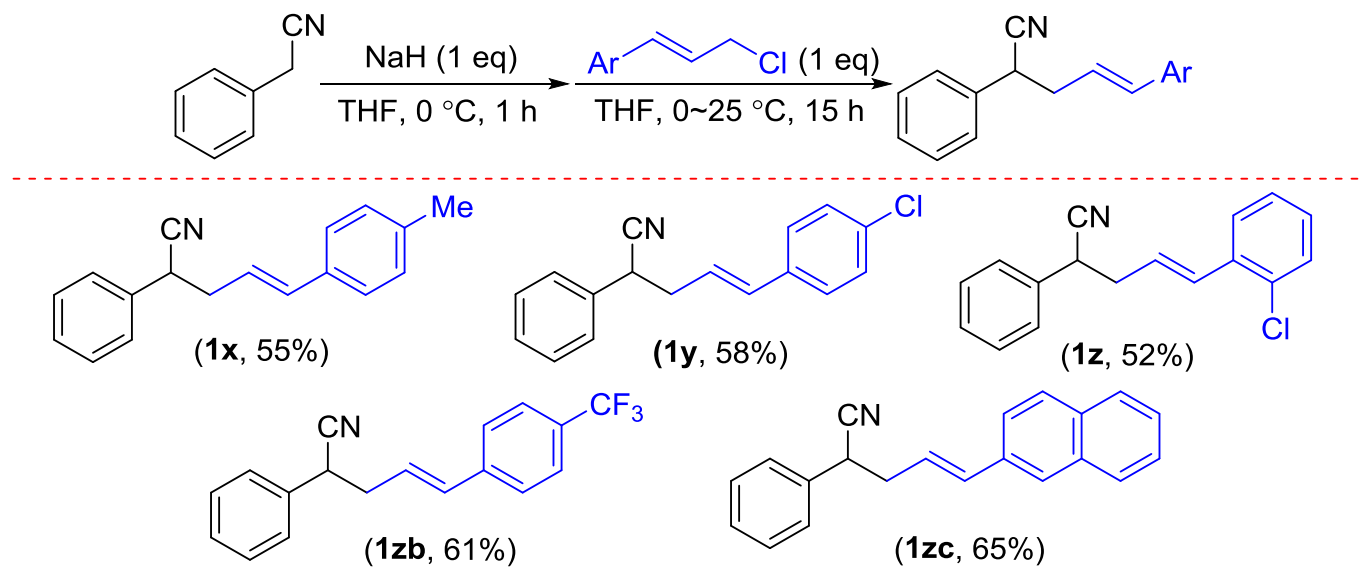

To a $200 \mathrm{~mL}$ Schlenk flask equipped with a stir bar was added vinylmagnesium bromide (1.0 $\mathrm{M}$ in THF, $30.0 \mathrm{~mL}, 30.0 \mathrm{mmol})$ and $\mathrm{THF}(20 \mathrm{~mL})$ at $0{ }^{\circ} \mathrm{C}$. Then a solution of the aromatic aldehyde in $\mathrm{THF}$ 
(25.0 mmol, $30 \mathrm{~mL}$ ) was added dropwise via a constant pressure dropping funnel and the resulting solution was stirred at $0{ }^{\circ} \mathrm{C}$ for two hours. The reaction was then quenched with sat. $\mathrm{NH}_{4} \mathrm{Cl}$ (aq.), and extracted into diethyl ether $(3 \times 60 \mathrm{~mL})$. The combined organic phases were washed with brine, dried over $\mathrm{Na}_{2} \mathrm{SO}_{4}$, filtered, and concentrated in vacuo. The residue was purified by chromatography on silica gel to afford 1-arylprop-2-en-1-ols.

To a $200 \mathrm{~mL}$ Schlenk flask equipped with a stir bar was added the above obtained 1-arylprop-2-en-1ol $(10.0 \mathrm{mmol})$ and THF $(20 \mathrm{~mL})$ under an atmosphere of argon at $0{ }^{\circ} \mathrm{C}$, followed by the dropwise addition of thionyl chloride (100 mmol, 10 equiv) via a constant pressure dropping funnel. The resulting solution was allowed to stir for $2 \mathrm{~h}$ before being quenched by pouring into ice cold brine $(20 \mathrm{~mL})$, and extracted with ice-cold $\mathrm{CH}_{2} \mathrm{Cl}_{2}(3 \times 50 \mathrm{~mL})$. The combined organic phases were concentrated under reduced pressure to afford cinnamyl chloride which was used without further purification.

Under an atmosphere of argon, a solution of 2-phenylacetonitrile (0.984 g, $8.4 \mathrm{mmol})$ in dry THF (5 $\mathrm{mL})$ was added slowly to a suspension of $\mathrm{NaH}(0.337 \mathrm{~g}, 8.4 \mathrm{mmol}, 60 \%$ dispersion in oil $)$ in dry THF $(15 \mathrm{~mL})$ at $0{ }^{\circ} \mathrm{C}$. The resulting mixture was stirred for $1 \mathrm{~h}$ and a solution of cinnamyl chloride $(8.4$ mmol) in dry THF $(20 \mathrm{~mL})$ was added dropwise at $0{ }^{\circ} \mathrm{C}$. The reaction was stirred at $0{ }^{\circ} \mathrm{C}$ for one hour and then was stirred at room temperature for 15 hours. The resulting solution was poured into cold water $(100 \mathrm{~mL})$ and extracted with ether $(3 \times 60 \mathrm{~mL})$. The combined organic extracts were washed with brine $(2 \times 50 \mathrm{~mL})$, dried over anhydrous $\mathrm{Na}_{2} \mathrm{SO}_{4}$, filtered and the filtrate was concentrated in vacuo. The residue was purified by column chromatography (silica gel, with $n$-hexane/ethyl acetate as eluent) and was further recrystallized from petroleum ether/ethyl acetate to afford $\mathbf{1 x ~ 1 z , ~ 1 z b , ~ 1 z c . ~}$

\section{Procedure $\mathbf{D}^{11,13}$ :}

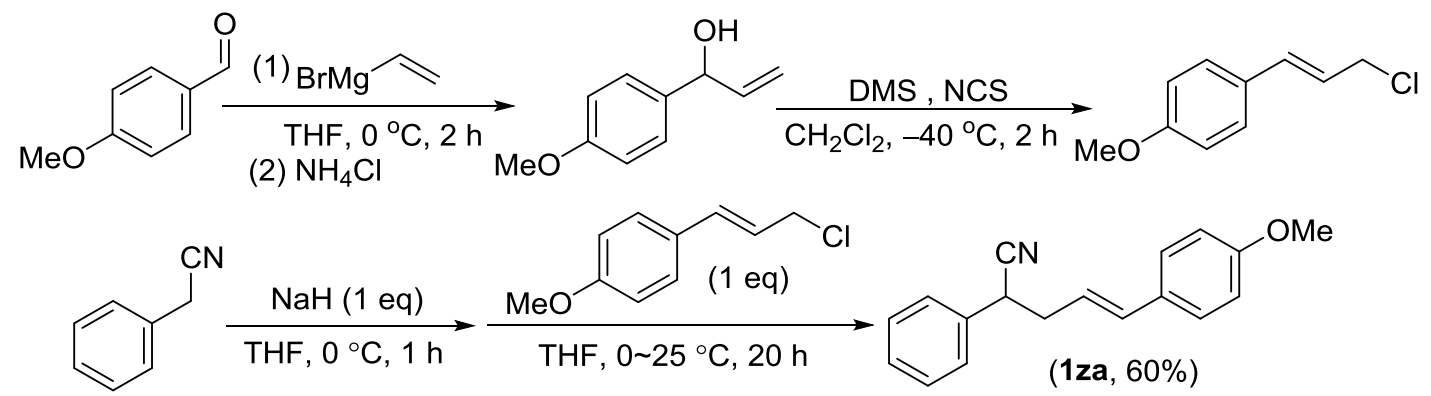

To a $200 \mathrm{~mL}$ Schlenk flask equipped with a stir bar was added (1.0 M in THF, $30.0 \mathrm{~mL}, 30.0 \mathrm{mmol})$ and THF $(20 \mathrm{~mL})$ at $0{ }^{\circ} \mathrm{C}$, followed by the dropwise addition of a solution of $p$-anisaldehyde $(4.35 \mathrm{~g}$, $25.0 \mathrm{mmol})$ in THF $(30 \mathrm{~mL})$ via a constant pressure dropping funnel. The reaction mixture was allowed to stir at $0{ }^{\circ} \mathrm{C}$ for 2 hours, then quenched with sat. $\mathrm{NH}_{4} \mathrm{Cl}$ (aq.), and extracted into diethyl ether $(3 \times 60$ $\mathrm{mL})$. The combined organic phases were washed with brine, dried over $\mathrm{Na}_{2} \mathrm{SO}_{4}$, filtered, and then concentrated in vacuo. The residue was purified by chromatography on silica gel to afford 1-(4methoxyphenyl)prop-2-en-1-ol.

To a $200 \mathrm{~mL}$ Schlenk flask equipped with a stir bar was added $N$-chlorosuccinimide (1.736 g, 13.0 mmol ) and $\mathrm{CH}_{2} \mathrm{Cl}_{2}(50 \mathrm{~mL})$ under an atmosphere of argon. The solution was then cooled to $-40{ }^{\circ} \mathrm{C}$ and dimethylsulfide (DMS, $1.2 \mathrm{~mL}, 16.0 \mathrm{mmol}$ ) was added dropwise vis syringe. The reaction was allowed to stir for one hour, at which point 1-(4-methoxyphenyl)prop-2-en-1-ol (1.64 g, $10.0 \mathrm{mmol}$ ) in $\mathrm{CH}_{2} \mathrm{Cl}_{2}$ $(50 \mathrm{~mL})$ was added dropwise via a constant pressure dropping funnel. The resulting solution was then warmed to $0^{\circ} \mathrm{C}$ and allowed to stir for $1 \mathrm{~h}$. The reaction was then diluted with brine (100 mL), extracted with $\mathrm{CH}_{2} \mathrm{Cl}_{2}(3 \times 100 \mathrm{~mL})$. The combined organic phases were concentrated under reduced pressure to 
afford $1.54 \mathrm{~g}$ (84\% yield) of a white solid that was used without further purification.

Under an atmosphere of argon, a solution of 2-phenylacetonitrile (0.984 g, $8.4 \mathrm{mmol})$ in dry THF (5 $\mathrm{mL})$ was added slowly to a suspension of $\mathrm{NaH}(0.337 \mathrm{~g}, 8.4 \mathrm{mmol}, 60 \%$ dispersion in oil $)$ in dry THF $(15 \mathrm{~mL})$ at $0{ }^{\circ} \mathrm{C}$. The resulting mixture was stirred for $1 \mathrm{~h}$ and a solution of $(E)$-1-(3-chloroprop-1-en-1yl)-4-methoxybenzene $(8.4 \mathrm{mmol})$ in dry THF $(20 \mathrm{~mL})$ was added dropwise and the resulting solution was stirred for another hour at $0{ }^{\circ} \mathrm{C}$. Then the reaction mixture was warmed to room temperature and stirred for 20 hours, poured into cold water $(100 \mathrm{~mL})$ and extracted with ether $(3 \times 60 \mathrm{~mL})$. The combined organic extracts were washed with brine $(2 \times 50 \mathrm{~mL})$, dried over anhydrous $\mathrm{Na}_{2} \mathrm{SO}_{4}$, and the filtrate was concentrated in vacuo and the residue was purified by column chromatography (silica gel, with $n$-hexane/ethyl acetate as eluent) to afford (E)-5-(4-methoxyphenyl)-2-phenylpent-4-enenitrile (1za, $1.32 \mathrm{~g}, 60 \%)$.

\section{Synthesis of Amine Substrates $2 t$ and $2 u^{14}$}

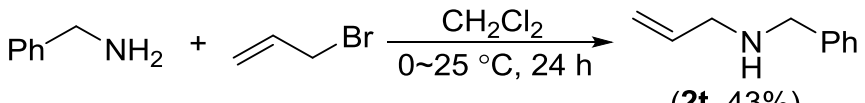

$(2 t, 43 \%)$

A solution of allyl bromide in dichloromethane $(20 \mathrm{mmol}, 2.4 \mathrm{~g}$ in $30 \mathrm{~mL})$ was slowly added to phenylmethanamine $(50 \mathrm{mmol}, 5.4 \mathrm{~g})$ at $0{ }^{\circ} \mathrm{C}$. Then, the solution was warmed to room temperature and stirred for 24 hours. The reaction was quenched with water $(50 \mathrm{~mL})$ and the resulting mixture was extracted with $\mathrm{CH}_{2} \mathrm{Cl}_{2}(50 \mathrm{~mL} \times 2)$. The combined organic layers were dried over $\mathrm{Na}_{2} \mathrm{SO}_{4}$, filtered, and concentrated. The residue was purified by column chromatography (silica gel, with hexane/ethyl acetate as eluent) to afford $\mathbf{2 t}(1.27 \mathrm{~g}, 43 \%$ yield).

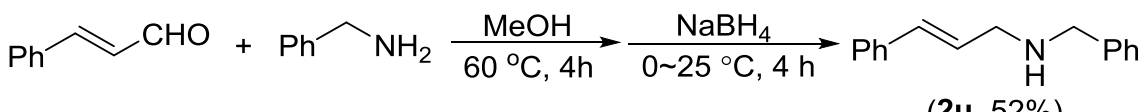

A mixture of cinnamaldehyde $(2.6 \mathrm{~g}, 20 \mathrm{mmol})$ and phenylmethanamine $(2.1 \mathrm{~g}, 20.0 \mathrm{mmol})$ in $\mathrm{MeOH}$ $(20 \mathrm{~mL})$ was heated at $60{ }^{\circ} \mathrm{C}$ for $4 \mathrm{~h}$ and was then cooled to $0{ }^{\circ} \mathrm{C} . \mathrm{NaBH}_{4}(2.3 \mathrm{~g}, 60 \mathrm{mmol})$ was added to the reaction mixture, and the solution was stirred at room temperature for $4 \mathrm{~h}$. After removal of the solvent, the solid residue obtained was dissolved in ethyl acetate $(50 \mathrm{~mL})$ and water $(50 \mathrm{~mL})$. The organic layer was orderly washed with saturated $\mathrm{NaHCO}_{3}(50 \mathrm{~mL})$, water $(50 \mathrm{~mL})$, and saturated $\mathrm{NaCl}$ $(50 \mathrm{~mL})$, and then dried over anhydrous $\mathrm{Na}_{2} \mathrm{SO}_{4}$, and concentrated. Column chromatography (silica gel, with $n$-hexane/ethyl acetate as eluent) of the residue afforded $2 \mathbf{u}(2.32 \mathrm{~g}, 52 \%)$.

\section{Characterization Data of Substrates 1}

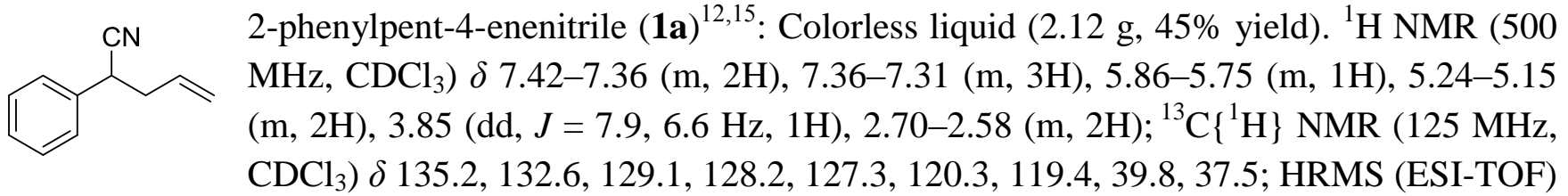
calcd for $\mathrm{C}_{11} \mathrm{H}_{12} \mathrm{~N}\left([\mathrm{M}+\mathrm{H}]^{+}\right)$158.0964, found 158.0967.

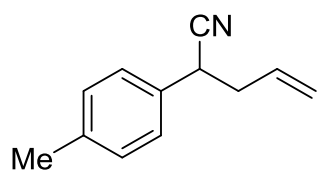

2-(p-tolyl)pent-4-enenitrile (1b $)^{15}$ : Colorless liquid $\left(2.41 \mathrm{~g}, 47 \%\right.$ yield). ${ }^{1} \mathrm{H}$ NMR $\left(500 \mathrm{MHz}, \mathrm{CDCl}_{3}\right) \delta 7.24-7.14(\mathrm{~m}, 4 \mathrm{H}), 5.87-5.74(\mathrm{~m}, 1 \mathrm{H}), 5.23-5.14(\mathrm{~m}, 2 \mathrm{H})$, $3.81(\mathrm{dd}, J=8.0,6.5 \mathrm{~Hz}, 1 \mathrm{H}), 2.68-2.55(\mathrm{~m}, 2 \mathrm{H}), 2.35(\mathrm{~s}, 3 \mathrm{H}) ;{ }^{13} \mathrm{C}\left\{{ }^{1} \mathrm{H}\right\} \mathrm{NMR}(125$ $\left.\mathrm{MHz}, \mathrm{CDCl}_{3}\right) \delta 138.0,132.7,132.2,129.7,127.2,120.5,119.2,39.9,37.1,21.0$; 
HRMS (ESI-TOF) calcd for $\mathrm{C}_{12} \mathrm{H}_{14} \mathrm{~N}\left([\mathrm{M}+\mathrm{H}]^{+}\right)$172.1121, found 172.1125.

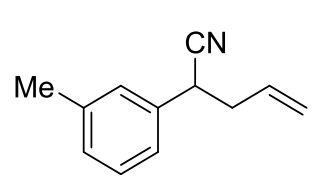

2-( $m$-tolyl)pent-4-enenitrile (1c) ${ }^{15}$ : Colorless liquid (2.26 g, 44\% yield). ${ }^{1} \mathrm{H}$ NMR $\left(500 \mathrm{MHz}, \mathrm{CDCl}_{3}\right) \delta 7.30-7.24(\mathrm{~m}, 1 \mathrm{H}), 7.18-7.09(\mathrm{~m}, 3 \mathrm{H}), 5.80$ (ddt, $J=17.2,10.2$, $7.0 \mathrm{~Hz}, 1 \mathrm{H}), 5.24-5.15(\mathrm{~m}, 2 \mathrm{H}), 3.81(\mathrm{dd}, J=8.2,6.4 \mathrm{~Hz}, 1 \mathrm{H}), 2.70-2.56(\mathrm{~m}, 2 \mathrm{H})$, $2.37(\mathrm{~s}, 3 \mathrm{H}) ;{ }^{13} \mathrm{C}\left\{{ }^{1} \mathrm{H}\right\}$ NMR $\left(125 \mathrm{MHz}, \mathrm{CDCl}_{3}\right) \delta 138.9,135.1,132.7,128.93$, $128.90,128.0,124.4,120.4,119.2,39.9,37.5,21.4$; HRMS (ESI-TOF) calcd for $\mathrm{C}_{12} \mathrm{H}_{14} \mathrm{~N}\left([\mathrm{M}+\mathrm{H}]^{+}\right)$ 172.1121, found 172.1117 .

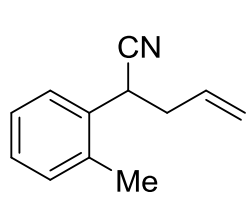

2-(o-tolyl)pent-4-enenitrile (1d) ${ }^{15}$ : Colorless liquid (2.16 g, 42\% yield). ${ }^{1} \mathrm{H}$ NMR (500 $\left.\mathrm{MHz}, \mathrm{CDCl}_{3}\right) \delta 7.44(\mathrm{dd}, J=7.2,1.8 \mathrm{~Hz}, 1 \mathrm{H}), 7.30-7.16(\mathrm{~m}, 3 \mathrm{H}), 5.85(\mathrm{ddt}, J=17.1$, $10.2,7.0 \mathrm{~Hz}, 1 \mathrm{H}), 5.27-5.17(\mathrm{~m}, 2 \mathrm{H}), 4.01(\mathrm{dd}, J=8.8,5.9 \mathrm{~Hz}, 1 \mathrm{H}), 2.68-2.52(\mathrm{~m}, 2 \mathrm{H})$, $2.36(\mathrm{~s}, 3 \mathrm{H}) ;{ }^{13} \mathrm{C}\left\{{ }^{1} \mathrm{H}\right\}$ NMR $\left(125 \mathrm{MHz}, \mathrm{CDCl}_{3}\right) \delta 134.9,133.6,132.8,131.0,128.2$, 127.5, 126.8, 120.6, 119.3, 38.5, 34.3, 19.2; HRMS (ESI-TOF) calcd for $\mathrm{C}_{12} \mathrm{H}_{14} \mathrm{~N}\left([\mathrm{M}+\mathrm{H}]^{+}\right) 172.1121$, found 172.1129 .

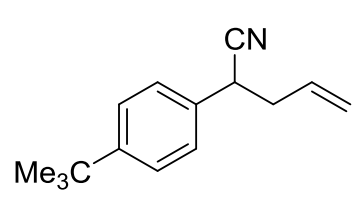

2-(4-(tert-butyl)phenyl)pent-4-enenitrile (1e): Colorless liquid (3.07 g, 48\% yield). ${ }^{1} \mathrm{H}$ NMR $\left(500 \mathrm{MHz}, \mathrm{CDCl}_{3}\right) \delta 7.40(\mathrm{~d}, J=8.4 \mathrm{~Hz}, 2 \mathrm{H}), 7.26(\mathrm{~d}, J=8.3$ $\mathrm{Hz}, 2 \mathrm{H}$ ), 5.82 (ddt, $J=17.1,10.2,7.0 \mathrm{~Hz}, 1 \mathrm{H}), 5.26-5.15(\mathrm{~m}, 2 \mathrm{H}), 3.82(\mathrm{dd}, J=$ 8.2, $6.4 \mathrm{~Hz}, 1 \mathrm{H}), 2.70-2.57(\mathrm{~m}, 2 \mathrm{H}), 1.32(\mathrm{~s}, 9 \mathrm{H}) ;{ }^{13} \mathrm{C}\left\{{ }^{1} \mathrm{H}\right\}$ NMR $(125 \mathrm{MHz}$, $\left.\mathrm{CDCl}_{3}\right) \delta 151.2,132.8,132.2,127.0,126.0,120.5,119.2,39.8,37.1,34.6,31.3$; HRMS (ESI-TOF) calcd for $\mathrm{C}_{15} \mathrm{H}_{20} \mathrm{~N}\left([\mathrm{M}+\mathrm{H}]^{+}\right)$214.1590, found 214.1588.

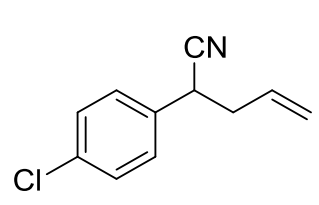

2-(4-chlorophenyl)pent-4-enenitrile (1f $)^{15}$ : Colorless liquid (2.88 g, $50 \%$ yield). ${ }^{1} \mathrm{H}$ NMR $\left(500 \mathrm{MHz}, \mathrm{CDCl}_{3}\right) \delta 7.42-7.33(\mathrm{~m}, 2 \mathrm{H}), 7.29-7.24(\mathrm{~m}, 2 \mathrm{H}), 5.77$ (ddt, $J=$ $17.2,10.3,7.0 \mathrm{~Hz}, 1 \mathrm{H}), 5.24-5.11(\mathrm{~m}, 2 \mathrm{H}), 3.87-3.80(\mathrm{~m}, 1 \mathrm{H}), 2.68-2.54(\mathrm{~m}, 2 \mathrm{H})$; ${ }^{13} \mathrm{C}\left\{{ }^{1} \mathrm{H}\right\}$ NMR $\left(125 \mathrm{MHz}, \mathrm{CDCl}_{3}\right) \delta 134.2,133.6,132.1,129.3,128.7,119.8,119.8$, 39.7, 36.9; HRMS (ESI-TOF) calcd for $\mathrm{C}_{11} \mathrm{H}_{11} \mathrm{CIN}\left([\mathrm{M}+\mathrm{H}]^{+}\right)$192.0575, found 192.0571.

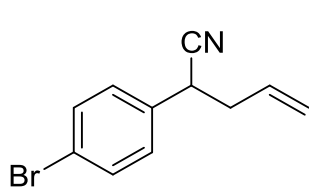

2-(4-bromophenyl)pent-4-enenitrile (19) ${ }^{[16]}$ : Colorless liquid (3.40 g, $48 \%$ yield).

${ }^{1} \mathrm{H}$ NMR $\left(500 \mathrm{MHz}, \mathrm{CDCl}_{3}\right) \delta 7.57-7.49(\mathrm{~m}, 2 \mathrm{H}), 7.25-7.16(\mathrm{~m}, 2 \mathrm{H}), 5.76$ (ddt, $J$ $=17.2,10.3,7.0 \mathrm{~Hz}, 1 \mathrm{H}), 5.25-5.12(\mathrm{~m}, 2 \mathrm{H}), 3.87-3.78(\mathrm{~m}, 1 \mathrm{H}), 2.69-2.54(\mathrm{~m}$, $2 \mathrm{H}) ;{ }^{13} \mathrm{C}\left\{{ }^{1} \mathrm{H}\right\}$ NMR $\left(125 \mathrm{MHz}, \mathrm{CDCl}_{3}\right) \delta 134.2,132.2,132.1,129.0,122.3,119.81$, 119.77, 39.6, 37.0; HRMS (ESI-TOF) calcd for $\mathrm{C}_{11} \mathrm{H}_{11} \mathrm{BrN}\left([\mathrm{M}+\mathrm{H}]^{+}\right)$236.0069, found 236.0070.

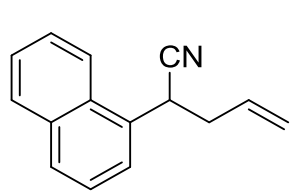

2-(naphthalen-1-yl)pent-4-enenitrile (1h $)^{17}$ : Light yellow liquid $(5.29 \mathrm{~g}, 85 \%$ yield). ${ }^{1} \mathrm{H}$ NMR $\left(500 \mathrm{MHz}, \mathrm{CDCl}_{3}\right) \delta 7.95-7.89(\mathrm{~m}, 2 \mathrm{H}), 7.85(\mathrm{~d}, J=8.3 \mathrm{~Hz}, 1 \mathrm{H}), 7.69(\mathrm{~d}$, $J=7.1 \mathrm{~Hz}, 1 \mathrm{H}), 7.63-7.47$ (m, 3H), 5.91 (ddt, $J=17.1,10.2,7.0 \mathrm{~Hz}, 1 \mathrm{H}), 5.29-5.19$ $(\mathrm{m}, 2 \mathrm{H}), 4.62(\mathrm{dd}, J=8.7,5.4 \mathrm{~Hz}, 1 \mathrm{H}), 2.86-2.70(\mathrm{~m}, 2 \mathrm{H}) ;{ }^{13} \mathrm{C}\left\{{ }^{1} \mathrm{H}\right\} \mathrm{NMR}(125 \mathrm{MHz}$, $\left.\mathrm{CDCl}_{3}\right) \delta 134.0,132.8,130.8,129.9,129.4,129.1,127.0,126.1,125.7,125.4,122.0,120.6,119.4,38.7$, 34.5; HRMS (ESI-TOF) calcd for $\mathrm{C}_{15} \mathrm{H}_{14} \mathrm{~N}\left([\mathrm{M}+\mathrm{H}]^{+}\right)$208.1121, found 208.1124.

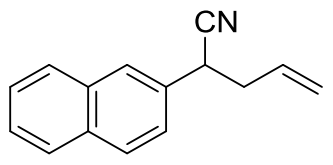

2-(naphthalen-2-yl)pent-4-enenitrile (1ii) ${ }^{18}$ : White solid (5.16 g, 83\% yield). Mp: $43-44^{\circ} \mathrm{C} ;{ }^{1} \mathrm{H}$ NMR $\left(500 \mathrm{MHz}, \mathrm{CDCl}_{3}\right) \delta 7.93-7.79(\mathrm{~m}, 4 \mathrm{H}), 7.59-7.48(\mathrm{~m}, 2 \mathrm{H})$, $7.41(\mathrm{dd}, J=8.5,1.9 \mathrm{~Hz}, 1 \mathrm{H}), 5.83(\mathrm{ddt}, J=17.1,10.2,7.0 \mathrm{~Hz}, 1 \mathrm{H}), 5.28-5.14(\mathrm{~m}$, $2 \mathrm{H}), 4.07-3.99(\mathrm{~m}, 1 \mathrm{H}), 2.80-2.66(\mathrm{~m}, 2 \mathrm{H}) ;{ }^{13} \mathrm{C}\left\{{ }^{1} \mathrm{H}\right\}$ NMR (125 MHz, $\left.\mathrm{CDCl}_{3}\right) \delta$ 
$133.3,132.8,132.6,132.5,129.1,127.9,127.8,126.8,126.6,126.5,124.8,120.3,119.5,39.8,37.7$; HRMS (ESI-TOF) calcd for $\mathrm{C}_{15} \mathrm{H}_{14} \mathrm{~N}\left([\mathrm{M}+\mathrm{H}]^{+}\right)$208.1121, found 208.1123.

CN 2-(4-methoxyphenyl)pent-4-enenitrile $(\mathbf{1 j})^{15}$ : Colorless liquid $(2.53 \mathrm{~g}, 45 \%$ yield).

${ }^{1} \mathrm{H}$ NMR $\left(500 \mathrm{MHz}, \mathrm{CDCl}_{3}\right) \delta 7.28-7.22(\mathrm{~m}, 2 \mathrm{H}), 6.94-6.87(\mathrm{~m}, 2 \mathrm{H}), 5.86-5.72$ $(\mathrm{m}, 1 \mathrm{H}), 5.22-5.15(\mathrm{~m}, 2 \mathrm{H}), 3.84-3.77(\mathrm{~m}, 4 \mathrm{H}), 2.68-2.54(\mathrm{~m}, 2 \mathrm{H}) ;{ }^{13} \mathrm{C}\left\{{ }^{1} \mathrm{H}\right\}$ NMR $\left(125 \mathrm{MHz}, \mathrm{CDCl}_{3}\right) \delta 159.4,132.7,128.5,127.2,120.6,119.3,114.4,55.3$, 39.9, 36.7; HRMS (ESI-TOF) calcd for $\mathrm{C}_{12} \mathrm{H}_{14} \mathrm{NO}\left([\mathrm{M}+\mathrm{H}]^{+}\right)$188.1070, found 188.1065.

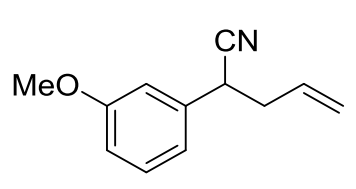

2-(3-methoxyphenyl)pent-4-enenitrile (1k): Colorless liquid (2.25 g, $40 \%$ yield). ${ }^{1} \mathrm{H}$ NMR $\left(500 \mathrm{MHz}, \mathrm{CDCl}_{3}\right) \delta$ 7.32-7.27 (m, 1H), 6.95-6.84 (m, 3H), 5.87-5.74 $(\mathrm{m}, 1 \mathrm{H}), 5.25-5.15(\mathrm{~m}, 2 \mathrm{H}), 3.87-3.78(\mathrm{~m}, 4 \mathrm{H}), 2.70-2.58(\mathrm{~m}, 2 \mathrm{H}) ;{ }^{13} \mathrm{C}\left\{{ }^{1} \mathrm{H}\right\}$ NMR $\left(125 \mathrm{MHz}, \mathrm{CDCl}_{3}\right) \delta 160.0,136.6,132.6,130.1,120.2,119.6,119.4,113.5$, 113.2, 55.3, 39.8, 37.5; HRMS (ESI-TOF) calcd for $\mathrm{C}_{12} \mathrm{H}_{14} \mathrm{NO}\left([\mathrm{M}+\mathrm{H}]^{+}\right)$188.1070, found 188.1075.

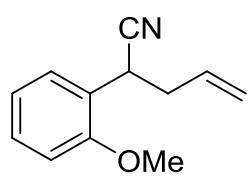

2-(2-methoxyphenyl)pent-4-enenitrile $(\mathbf{1 1})^{19}$ : Colorless liquid $(2.36 \mathrm{~g}, 42 \%$ yield $) .{ }^{1} \mathrm{H}$ $\operatorname{NMR}\left(500 \mathrm{MHz}, \mathrm{CDCl}_{3}\right) \delta 7.40(\mathrm{dd}, J=7.6,1.7 \mathrm{~Hz}, 1 \mathrm{H}), 7.30(\mathrm{ddd}, J=8.2,7.6,1.7$ $\mathrm{Hz}, 1 \mathrm{H}), 6.98(\mathrm{td}, J=7.5,1.1 \mathrm{~Hz}, 1 \mathrm{H}), 6.90(\mathrm{~d}, J=8.3 \mathrm{~Hz}, 1 \mathrm{H}), 5.83(\mathrm{ddt}, J=17.2$, $10.2,7.0 \mathrm{~Hz}, 1 \mathrm{H}), 5.24-5.13(\mathrm{~m}, 2 \mathrm{H}), 4.26(\mathrm{dd}, J=8.0,6.2 \mathrm{~Hz}, 1 \mathrm{H}), 3.86(\mathrm{~s}, 3 \mathrm{H})$, 2.65-2.55 (m, 2H); ${ }^{13} \mathrm{C}\left\{{ }^{1} \mathrm{H}\right\}$ NMR (125 MHz, $\left.\mathrm{CDCl}_{3}\right) \delta 156.0,133.2,129.4,128.5,123.5,120.9,120.7$, 118.8, 110.8, 55.5, 37.6, 31.7; HRMS (ESI-TOF) calcd for $\mathrm{C}_{12} \mathrm{H}_{14} \mathrm{NO}\left([\mathrm{M}+\mathrm{H}]^{+}\right) 188.1070$, found 188.1072 .

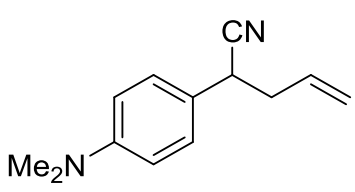

2-(4-(dimethylamino)phenyl)pent-4-enenitrile (1m): Light yellow liquid (3.12 g, $52 \%$ yield). ${ }^{1} \mathrm{H}$ NMR $\left(500 \mathrm{MHz} \mathrm{CDCl}_{3}\right) \delta$ 7.21-7.14 (m, 2H), 6.74-6.66 (m, $2 \mathrm{H}), 5.81$ (ddt, $J=17.1,10.2,7.0 \mathrm{~Hz}, 1 \mathrm{H}), 5.23-5.13(\mathrm{~m}, 2 \mathrm{H}), 3.76(\mathrm{dd}, J=8.2$, $6.4 \mathrm{~Hz}, 1 \mathrm{H}), 2.96(\mathrm{~s}, 6 \mathrm{H}), 2.66-2.53(\mathrm{~m}, 2 \mathrm{H}) ;{ }^{13} \mathrm{C}\left\{{ }^{1} \mathrm{H}\right\} \mathrm{NMR}\left(125 \mathrm{MHz}, \mathrm{CDCl}_{3}\right)$ $\delta$ 150.2, 133.1, 128.0, 122.5, 120.9, 119.0, 112.6, 40.5, 40.0, 36.6; HRMS (ESI-TOF) calcd for $\mathrm{C}_{13} \mathrm{H}_{17} \mathrm{~N}_{2}\left([\mathrm{M}+\mathrm{H}]^{+}\right)$201.1386, found 201.1390.

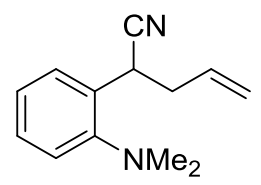

2-(2-(dimethylamino)phenyl)pent-4-enenitrile (1n): Colorless liquid (2.70 g, 45\% yield). ${ }^{1} \mathrm{H}$ NMR $\left(500 \mathrm{MHz}, \mathrm{CDCl}_{3}\right) \delta 7.44(\mathrm{dd}, J=7.7,1.6 \mathrm{~Hz}, 1 \mathrm{H}), 7.30$ (ddd, $J=8.1$, $7.3,1.6 \mathrm{~Hz}, 1 \mathrm{H}), 7.28-7.20(\mathrm{~m}, 1 \mathrm{H}), 7.18-7.12(\mathrm{~m}, 1 \mathrm{H}), 5.83(\mathrm{ddt}, J=17.1,10.2,7.0$ $\mathrm{Hz}, 1 \mathrm{H}), 5.22-5.10(\mathrm{~m}, 2 \mathrm{H}), 4.56(\mathrm{dd}, J=8.8,6.1 \mathrm{~Hz}, 1 \mathrm{H}), 2.71-2.60(\mathrm{~m}, 7 \mathrm{H}), 2.59-$ $2.50(\mathrm{~m}, 1 \mathrm{H}) ;{ }^{13} \mathrm{C}\left\{{ }^{1} \mathrm{H}\right\} \mathrm{NMR}\left(125 \mathrm{MHz}, \mathrm{CDCl}_{3}\right) \delta 152.2,133.4,131.7,129.1,128.3,125.0,121.6,121.4$, 118.6, 45.8, 39.1, 31.3; HRMS (ESI-TOF) calcd for $\mathrm{C}_{13} \mathrm{H}_{17} \mathrm{~N}_{2}\left([\mathrm{M}+\mathrm{H}]^{+}\right)$201.1386, found 201.1393.

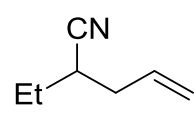

2-ethylpent-4-enenitrile (1o $)^{20}$ : Colorless liquid (1.24 g, 38\% yield). ${ }^{1} \mathrm{H}$ NMR $(500 \mathrm{MHz}$, $\left.\mathrm{CDCl}_{3}\right) \delta 5.81(\mathrm{ddt}, \mathrm{J}=17.2,10.2,7.0 \mathrm{~Hz}, 1 \mathrm{H}), 5.24-5.14(\mathrm{~m}, 2 \mathrm{H}), 2.58-2.49(\mathrm{~m}, 1 \mathrm{H})$, 2.41-2.29 (m, 2H), 1.70-1.57 (m, 2H), $1.08(\mathrm{t}, J=7.4 \mathrm{~Hz}, 3 \mathrm{H}) ;{ }^{13} \mathrm{C}\left\{{ }^{1} \mathrm{H}\right\} \mathrm{NMR}(125 \mathrm{MHz}$, $\left.\mathrm{CDCl}_{3}\right) \delta 133.2,121.6,118.7,35.9,33.1,25.0,11.5$; HRMS (ESI-TOF) calcd for $\mathrm{C}_{7} \mathrm{H}_{11} \mathrm{NNa}\left([\mathrm{M}+\mathrm{Na}]^{+}\right)$ 132.0784, found 132.0781 .

2-benzylpent-4-enenitrile $(1 \mathbf{p})^{21}$ : Colorless liquid $(2.16 \mathrm{~g}, 42 \%$ yield $) .{ }^{1} \mathrm{H}$ NMR $(500 \mathrm{MHz}$,
$\left.\mathrm{CDCl}_{3}\right) \delta 7.37-7.32(\mathrm{~m}, 2 \mathrm{H}), 7.30-7.22(\mathrm{~m}, 3 \mathrm{H}), 5.93-5.79(\mathrm{~m}, 1 \mathrm{H}), 5.25-5.18(\mathrm{~m}, 2 \mathrm{H})$,
$2.97-2.80(\mathrm{~m}, 3 \mathrm{H}), 2.44-2.30(\mathrm{~m}, 2 \mathrm{H}) ;{ }^{13} \mathrm{C}\left\{{ }^{1} \mathrm{H}\right\} \mathrm{NMR}\left(125 \mathrm{MHz}, \mathrm{CDCl}_{3}\right) \delta 136.8,132.9$, 
129.0, 128.8, 127.3, 121.2, 119.3, 37.7, 35.7, 33.6; HRMS (ESI-TOF) calcd for $\mathrm{C}_{12} \mathrm{H}_{14} \mathrm{~N}\left([\mathrm{M}+\mathrm{H}]^{+}\right)$ 172.1121, found 172.1129 .

CN 2-((dimethylamino)methyl)pent-4-enenitrile (1q): Colorless liquid (1.66 g, $40 \%$ yield). ${ }^{1} \mathrm{H}$ NMR $\left(500 \mathrm{MHz}, \mathrm{CDCl}_{3}\right) \delta 5.84(\mathrm{ddt}, J=17.1,10.2,7.0 \mathrm{~Hz}, 1 \mathrm{H}), 5.25-5.15(\mathrm{~m}, 2 \mathrm{H}), 2.79-$ $\mathrm{NMe}_{2} \quad 2.70(\mathrm{~m}, 1 \mathrm{H}), 2.58(\mathrm{dd}, J=12.4,8.4 \mathrm{~Hz}, 1 \mathrm{H}), 2.46-2.31(\mathrm{~m}, 3 \mathrm{H}), 2.28(\mathrm{~s}, 6 \mathrm{H}) ;{ }^{13} \mathrm{C}\left\{{ }^{1} \mathrm{H}\right\}$
$\mathrm{NMR}(125 \mathrm{MHz}, \mathrm{CDCl})$ $\mathrm{C}_{8} \mathrm{H}_{15} \mathrm{~N}_{2}\left([\mathrm{M}+\mathrm{H}]^{+}\right)$139.1230, found 139.1232 .

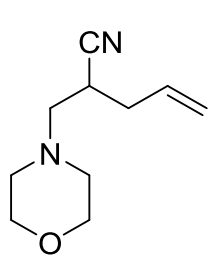

2-(morpholinomethyl)pent-4-enenitrile (1r): Colorless liquid $\left(2.43 \mathrm{~g}, 45 \%\right.$ yield). ${ }^{1} \mathrm{H}$ NMR $\left(500 \mathrm{MHz}, \mathrm{CDCl}_{3}\right) \delta 5.82$ (ddt, $\left.J=17.2,10.2,7.0 \mathrm{~Hz}, 1 \mathrm{H}\right), 5.25-5.15(\mathrm{~m}, 2 \mathrm{H})$, 3.74-3.66 (m, 4H), 2.82-2.73 (m, 1H), 2.66-2.59 (m, 1H), 2.54-2.44 (m, 5H), 2.44-2.31 $(\mathrm{m}, 2 \mathrm{H}) ;{ }^{13} \mathrm{C}\left\{{ }^{1} \mathrm{H}\right\}$ NMR $\left(125 \mathrm{MHz}, \mathrm{CDCl}_{3}\right) \delta 132.8,121.1,119.1,66.8,59.2,53.7,34.1$, 29.9; HRMS (ESI-TOF) calcd for $\mathrm{C}_{10} \mathrm{H}_{17} \mathrm{~N}_{2} \mathrm{O}\left([\mathrm{M}+\mathrm{H}]^{+}\right)$181.1335, found 181.1338.

2-(thiophen-2-yl)pent-4-enenitrile $(\mathbf{1 s})^{22}$ : Light yellow liquid $\left(2.69 \mathrm{~g}, 55 \%\right.$ yield). ${ }^{1} \mathrm{H}$

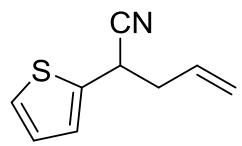

NMR $\left(500 \mathrm{MHz}, \mathrm{CDCl}_{3}\right) \delta 7.28(\mathrm{dd}, J=5.2,1.1 \mathrm{~Hz}, 1 \mathrm{H}), 7.08(\mathrm{~d}, J=3.5 \mathrm{~Hz}, 1 \mathrm{H}), 6.98$ $(\mathrm{dd}, J=5.1,3.6 \mathrm{~Hz}, 1 \mathrm{H}), 5.84(\mathrm{ddt}, J=17.1,10.2,7.0 \mathrm{~Hz}, 1 \mathrm{H}), 5.29-5.18(\mathrm{~m}, 2 \mathrm{H}), 4.14$ $(\mathrm{dd}, J=7.1,7.1 \mathrm{~Hz}, 1 \mathrm{H}), 2.72(\mathrm{dd}, J=7.1,7.1 \mathrm{~Hz}, 2 \mathrm{H}) ;{ }^{13} \mathrm{C}\left\{{ }^{1} \mathrm{H}\right\} \mathrm{NMR}(125 \mathrm{MHz}$, $\left.\mathrm{CDCl}_{3}\right) \delta 137.2,132.1,127.1,126.3,125.6,119.9,119.4,39.8,32.7$; HRMS (ESI-TOF) calcd for $\mathrm{C}_{9} \mathrm{H}_{10} \mathrm{NS}\left([\mathrm{M}+\mathrm{H}]^{+}\right)$164.0528, found 164.0529 .

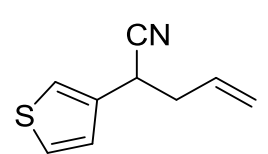

2-(thiophen-3-yl)pent-4-enenitrile (1t): Light yellow liquid $\left(2.79 \mathrm{~g}, 57 \%\right.$ yield). ${ }^{1} \mathrm{H}$ NMR $\left(500 \mathrm{MHz}, \mathrm{CDCl}_{3}\right) \delta 7.36(\mathrm{dd}, J=5.0,3.0 \mathrm{~Hz}, 1 \mathrm{H}), 7.26(\mathrm{~d}, J=1.7 \mathrm{~Hz}, 1 \mathrm{H})$, $7.05(\mathrm{dd}, J=5.0,1.2 \mathrm{~Hz}, 1 \mathrm{H}), 5.81$ (ddt, $J=17.2,10.3,7.0 \mathrm{~Hz}, 1 \mathrm{H}), 5.25-5.17(\mathrm{~m}$, 2H), $3.97(\mathrm{dd}, J=7.1,7.1 \mathrm{~Hz}, 1 \mathrm{H}), 2.65(\mathrm{dd}, J=7.0,7.0 \mathrm{~Hz}, 2 \mathrm{H}) ;{ }^{13} \mathrm{C}\left\{{ }^{1} \mathrm{H}\right\} \mathrm{NMR}\left(125 \mathrm{MHz}, \mathrm{CDCl}_{3}\right) \delta$ 135.2, 132.5, 127.2, 126.2, 122.7, 120.1, 119.5, 38.7, 32.8; HRMS (ESI-TOF) calcd for $\mathrm{C}_{9} \mathrm{H}_{10} \mathrm{NS}([\mathrm{M}+$ $\mathrm{H}]^{+}$) 164.0528 , found 164.0531 .

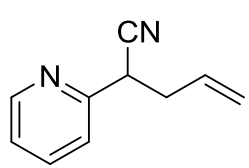

2-(pyridin-2-yl)pent-4-enenitrile (1u): Brown liquid (3.65 g, 77\% yield). ${ }^{1} \mathrm{H}$ NMR (500 $\left.\mathrm{MHz}, \mathrm{CDCl}_{3}\right) \delta 8.60(\mathrm{~d}, J=4.7 \mathrm{~Hz}, 1 \mathrm{H}), 7.73(\mathrm{ddd}, J=7.7,7.7,1.8 \mathrm{~Hz}, 1 \mathrm{H}), 7.43(\mathrm{~d}, J$ $=7.8 \mathrm{~Hz}, 1 \mathrm{H}), 7.26(\mathrm{dd}, J=6.2,6.2 \mathrm{~Hz} 1 \mathrm{H}), 5.82(\mathrm{ddt}, J=17.2,10.2,7.1 \mathrm{~Hz}, 1 \mathrm{H})$, 5.25-5.11 (m, 2H), $4.05(\mathrm{dd}, J=8.3,5.9 \mathrm{~Hz}, 1 \mathrm{H}), 2.87-2.66(\mathrm{~m}, 2 \mathrm{H}) ;{ }^{13} \mathrm{C}\left\{{ }^{1} \mathrm{H}\right\} \mathrm{NMR}\left(125 \mathrm{MHz}, \mathrm{CDCl}_{3}\right)$ $\delta$ 154.5, 149.9, 137.3, 132.5, 123.0, 121.9, 119.6, 119.5, 39.8, 38.0; HRMS (ESI-TOF) calcd for $\mathrm{C}_{10} \mathrm{H}_{11} \mathrm{~N}_{2}\left([\mathrm{M}+\mathrm{H}]^{+}\right)$159.0917, found 159.0911 .

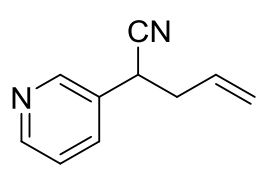

2-(pyridin-3-yl)pent-4-enenitrile (1v): Yellow liquid (3.42 g, 72\% yield). ${ }^{1} \mathrm{H}$ NMR $\left(500 \mathrm{MHz}, \mathrm{CDCl}_{3}\right) \delta 8.64-8.53(\mathrm{~m}, 2 \mathrm{H}), 7.75-7.66(\mathrm{~m}, 1 \mathrm{H}), 7.33(\mathrm{dd}, J=7.9,4.8 \mathrm{~Hz}$, $1 \mathrm{H}), 5.86-5.70(\mathrm{~m}, 1 \mathrm{H}), 5.25-5.12(\mathrm{~m}, 2 \mathrm{H}), 3.95-3.86(\mathrm{~m}, 1 \mathrm{H}), 2.73-2.57(\mathrm{~m}, 2 \mathrm{H})$; ${ }^{13} \mathrm{C}\left\{{ }^{1} \mathrm{H}\right\}$ NMR $\left(125 \mathrm{MHz}, \mathrm{CDCl}_{3}\right) \delta 149.7,148.8,134.9,131.7,131.0,123.8,120.3,119.3,39.5,35.1$; HRMS (ESI-TOF) calcd for $\mathrm{C}_{10} \mathrm{H}_{11} \mathrm{~N}_{2}\left([\mathrm{M}+\mathrm{H}]^{+}\right)$159.0917, found 159.0915.

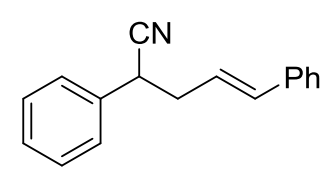

(E)-2,5-diphenylpent-4-enenitrile $(\mathbf{1 w})^{18}$ : White solid (2.45 g, 35\% yield). Mp: $43-$ $44^{\circ} \mathrm{C} ;{ }^{1} \mathrm{H}$ NMR $\left(500 \mathrm{MHz}, \mathrm{CDCl}_{3}\right) \delta 7.44-7.20(\mathrm{~m}, 10 \mathrm{H}), 6.51(\mathrm{~d}, J=15.8 \mathrm{~Hz}, 1 \mathrm{H})$, $6.23-6.12(\mathrm{~m}, 1 \mathrm{H}), 3.92(\mathrm{dd}, J=7.8,6.5 \mathrm{~Hz}, 1 \mathrm{H}), 2.88-2.73(\mathrm{~m}, 2 \mathrm{H}) ;{ }^{13} \mathrm{C}\left\{{ }^{1} \mathrm{H}\right\}$ NMR $\left(125 \mathrm{MHz}, \mathrm{CDCl}_{3}\right) \delta 136.6,135.2,134.4,129.1,128.6,128.2,127.8,127.4,126.4,123.8,120.3$, 39.2, 38.0; HRMS (ESI-TOF) calcd for $\mathrm{C}_{17} \mathrm{H}_{16} \mathrm{~N}\left([\mathrm{M}+\mathrm{H}]^{+}\right)$234.1277, found 234.1271. 


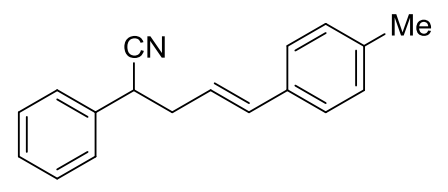

(E)-2-phenyl-5-(p-tolyl)pent-4-enenitrile (1x): White solid (1.21 g, 55\% yield). Mp: $52-53{ }^{\circ} \mathrm{C} ;{ }^{1} \mathrm{H}$ NMR $\left(500 \mathrm{MHz}, \mathrm{CDCl}_{3}\right) \delta 7.43-7.30(\mathrm{~m}, 5 \mathrm{H})$, $7.25-7.20(\mathrm{~m}, 2 \mathrm{H}), 7.11(\mathrm{~d}, J=7.4 \mathrm{~Hz}, 2 \mathrm{H}), 6.46(\mathrm{~d}, J=15.7 \mathrm{~Hz}, 1 \mathrm{H})$, 6.17-6.06 (m, 1H), $3.91(\mathrm{dd}, J=7.9,6.5 \mathrm{~Hz}, 1 \mathrm{H}), 2.85-2.72(\mathrm{~m}, 2 \mathrm{H}), 2.33$ (s, 3H); ${ }^{13} \mathrm{C}\left\{{ }^{1} \mathrm{H}\right\}$ NMR $\left(125 \mathrm{MHz}, \mathrm{CDCl}_{3}\right) \delta$ 137.6, 135.2, 134.2, 133.8, 129.3, 129.1, 128.2, 127.4, 126.3, 122.8, 120.4, 39.3, 38.1, 21.2; HRMS (ESI-TOF) calcd for $\mathrm{C}_{18} \mathrm{H}_{18} \mathrm{~N}\left([\mathrm{M}+\mathrm{H}]^{+}\right) 248.1434$, found 248.1424 .

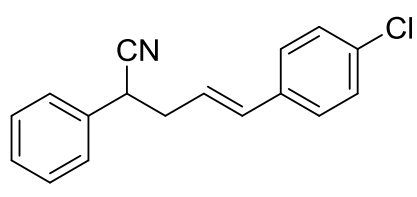

(E)-5-(4-chlorophenyl)-2-phenylpent-4-enenitrile (1y): White solid (1.43 g, $58 \%$ yield). Mp: $81-82{ }^{\circ} \mathrm{C} ;{ }^{1} \mathrm{H}$ NMR $\left(500 \mathrm{MHz}, \mathrm{CDCl}_{3}\right) \delta 7.39-7.24(\mathrm{~m}, 5 \mathrm{H})$, 7.23-7.14 (m, 4H), $6.37(\mathrm{~d}, J=15.8 \mathrm{~Hz}, 1 \mathrm{H}), 6.12-6.00(\mathrm{~m}, 1 \mathrm{H}), 3.85(\mathrm{t}, J=$ $7.1 \mathrm{~Hz}, 1 \mathrm{H}), 2.77-2.65(\mathrm{~m}, 2 \mathrm{H}) ;{ }^{13} \mathrm{C}\left\{{ }^{1} \mathrm{H}\right\}$ NMR $\left(125 \mathrm{MHz}, \mathrm{CDCl}_{3}\right) \delta 135.0$, 135.0, 133.3, 133.1, 129.1, 128.7, 128.2, 127.5, 127.3, 124.4, 120.2, 39.1, 37.8; HRMS (ESI-TOF) calcd for $\mathrm{C}_{17} \mathrm{H}_{15} \mathrm{ClN}\left([\mathrm{M}+\mathrm{H}]^{+}\right)$268.0888, found 268.0894 .

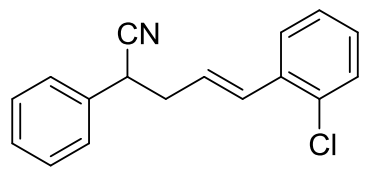

(E)-5-(2-chlorophenyl)-2-phenylpent-4-enenitrile (1z): White solid (1.28 g, 52\% yield). Mp: $63-64^{\circ} \mathrm{C} ;{ }^{1} \mathrm{H}$ NMR $\left(500 \mathrm{MHz}, \mathrm{CDCl}_{3}\right) \delta 7.51-7.30(\mathrm{~m}, 7 \mathrm{H}), 7.24-$ $7.13(\mathrm{~m}, 2 \mathrm{H}), 6.88(\mathrm{~d}, J=15.7 \mathrm{~Hz}, 1 \mathrm{H}), 6.21-6.10(\mathrm{~m}, 1 \mathrm{H}), 3.95(\mathrm{t}, J=7.2 \mathrm{~Hz}$, $1 \mathrm{H}), 2.92-2.76(\mathrm{~m}, 2 \mathrm{H}) ;{ }^{13} \mathrm{C}\left\{{ }^{1} \mathrm{H}\right\}$ NMR $\left(125 \mathrm{MHz}, \mathrm{CDCl}_{3}\right) \delta 135.0,134.8$, 132.9, 130.7, 129.7, 129.2, 128.8, 128.3, 127.4, 127.0, 126.9, 126.8, 120.2, 39.2, 37.8; HRMS (ESITOF) calcd for $\mathrm{C}_{17} \mathrm{H}_{15} \mathrm{ClN}\left([\mathrm{M}+\mathrm{H}]^{+}\right)$268.0888, found 268.0897.

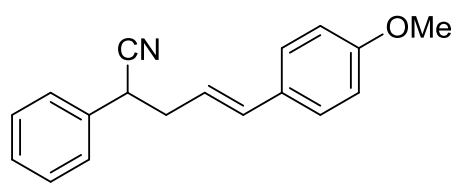

(E)-5-(4-methoxyphenyl)-2-phenylpent-4-enenitrile (1za) $)^{17,18}$ : White solid (1.32 g, 60\% yield). Mp: $83-84^{\circ} \mathrm{C} ;{ }^{1} \mathrm{H}$ NMR $\left(500 \mathrm{MHz}, \mathrm{CDCl}_{3}\right) \delta 7.47-$ $7.31(\mathrm{~m}, 5 \mathrm{H}), 7.27(\mathrm{~d}, J=8.6 \mathrm{~Hz}, 2 \mathrm{H}), 6.84(\mathrm{~d}, J=8.3 \mathrm{~Hz}, 2 \mathrm{H}), 6.44(\mathrm{~d}, J$ $=15.7 \mathrm{~Hz}, 1 \mathrm{H}), 6.08-5.97(\mathrm{~m}, 1 \mathrm{H}), 3.90(\mathrm{t}, J=7.1 \mathrm{~Hz}, 1 \mathrm{H}), 3.80(\mathrm{~s}, 3 \mathrm{H})$, 2.83-2.69 (m, 2H); ${ }^{13} \mathrm{C}\left\{{ }^{1} \mathrm{H}\right\}$ NMR $\left(125 \mathrm{MHz}, \mathrm{CDCl}_{3}\right) \delta 159.3,135.3,133.7,129.4,129.1,128.2,127.54$, 127.39, 121.6, 120.5, 114.0, 55.3, 39.3, 38.2; HRMS (ESI-TOF) calcd for $\mathrm{C}_{18} \mathrm{H}_{18} \mathrm{NO}\left([\mathrm{M}+\mathrm{H}]^{+}\right)$ 264.1383, found 264.1389.

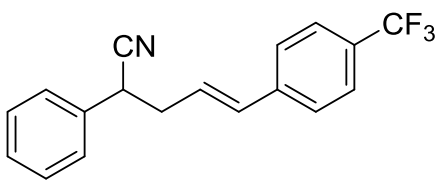

(E)-2-phenyl-5-(4-(trifluoromethyl)phenyl)pent-4-enenitrile (1zb): White solid (1.65 g, 61\% yield). Mp: $81-82^{\circ} \mathrm{C} ;{ }^{1} \mathrm{H}$ NMR $\left(500 \mathrm{MHz}, \mathrm{CDCl}_{3}\right) \delta 7.55$ (d, $J=8.1 \mathrm{~Hz}, 2 \mathrm{H}), 7.47-7.30(\mathrm{~m}, 7 \mathrm{H}), 6.53(\mathrm{~d}, J=15.8 \mathrm{~Hz}, 1 \mathrm{H}), 6.32-6.21$ $(\mathrm{m}, 1 \mathrm{H}), 3.96(\mathrm{t}, J=7.1 \mathrm{~Hz}, 1 \mathrm{H}), 2.89-2.75(\mathrm{~m}, 2 \mathrm{H}) ;{ }^{13} \mathrm{C}\left\{{ }^{1} \mathrm{H}\right\}$ NMR $(125$ $\left.\mathrm{MHz}, \mathrm{CDCl}_{3}\right) \delta 140.0,134.9,133.2,129.6\left(\mathrm{q},{ }^{2} J_{\mathrm{FC}}=32.5 \mathrm{~Hz}\right), 129.2,128.4,127.3,126.6,126.5,125.6$ $\left(\mathrm{q},{ }^{3} J_{\mathrm{FC}}=3.7 \mathrm{~Hz}\right), 124.1\left(\mathrm{q},{ }^{1} J_{\mathrm{FC}}=271.4 \mathrm{~Hz}\right), 120.1,39.1,37.8 ;{ }^{19} \mathrm{~F}\left\{{ }^{1} \mathrm{H}\right\} \mathrm{NMR}\left(470 \mathrm{MHz}, \mathrm{CDCl}_{3}\right) \delta-$ 62.5; HRMS (ESI-TOF) calcd for $\mathrm{C}_{18} \mathrm{H}_{14} \mathrm{~F}_{3} \mathrm{NNa}\left([\mathrm{M}+\mathrm{Na}]^{+}\right)$324.0971, found 324.0969.

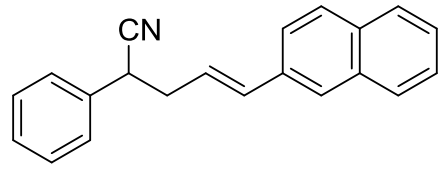

(E)-5-(naphthalen-2-yl)-2-phenylpent-4-enenitrile (1zc): White solid (1.68 g, $65 \%$ yield). $\mathrm{Mp}: 109-110^{\circ} \mathrm{C} ;{ }^{1} \mathrm{H}$ NMR $\left(500 \mathrm{MHz}, \mathrm{CDCl}_{3}\right) \delta 7.87-7.73$ $(\mathrm{m}, 3 \mathrm{H}), 7.70(\mathrm{~s}, 1 \mathrm{H}), 7.56(\mathrm{~d}, J=8.5 \mathrm{~Hz}, 1 \mathrm{H}), 7.51-7.29(\mathrm{~m}, 7 \mathrm{H}), 6.67(\mathrm{~d}$, $J=15.7 \mathrm{~Hz}, 1 \mathrm{H}), 6.35-6.24(\mathrm{~m}, 1 \mathrm{H}), 3.96(\mathrm{t}, J=7.1 \mathrm{~Hz}, 1 \mathrm{H}), 2.93-2.75(\mathrm{~m}$, $2 \mathrm{H}) ;{ }^{13} \mathrm{C}\left\{{ }^{1} \mathrm{H}\right\} \mathrm{NMR}\left(125 \mathrm{MHz}, \mathrm{CDCl}_{3}\right) \delta 135.2,134.5,134.1,133.6,133.1,129.2,128.3,128.0,127.7$, $127.4,126.4,126.0,124.2,123.5,120.4,39.4,38.0$; HRMS (ESI-TOF) calcd for $\mathrm{C}_{21} \mathrm{H}_{18} \mathrm{~N}\left([\mathrm{M}+\mathrm{H}]^{+}\right)$ 284.1434, found 284.1425 . 


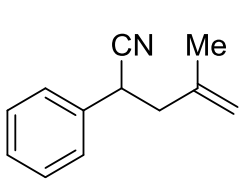

4-methyl-2-phenylpent-4-enenitrile (1zd $)^{23}$ : Colorless liquid (2.36 g, 46\% yield). ${ }^{1} \mathrm{H}$ NMR $\left(500 \mathrm{MHz}, \mathrm{CDCl}_{3}\right) \delta 7.43-7.28(\mathrm{~m}, 5 \mathrm{H}), 4.94(\mathrm{~s}, 1 \mathrm{H}), 4.86(\mathrm{~s}, 1 \mathrm{H}), 3.93(\mathrm{dd}, J=$ 9.6, 6.1 Hz, 1H), $2.64(\mathrm{dd}, J=14.3,9.6 \mathrm{~Hz}, 1 \mathrm{H}), 2.51(\mathrm{dd}, J=14.2,6.0 \mathrm{~Hz}, 1 \mathrm{H}), 1.78$ $(\mathrm{s}, 3 \mathrm{H}) ;{ }^{13} \mathrm{C}\left\{{ }^{1} \mathrm{H}\right\}$ NMR $\left(125 \mathrm{MHz}, \mathrm{CDCl}_{3}\right) \delta 140.2,135.7,129.1,128.1,127.3,120.5$, 114.7, 44.0, 36.3, 22.2; HRMS (ESI-TOF) calcd for $\mathrm{C}_{12} \mathrm{H}_{14} \mathrm{~N}\left([\mathrm{M}+\mathrm{H}]^{+}\right)$172.1121, found 172.1115.

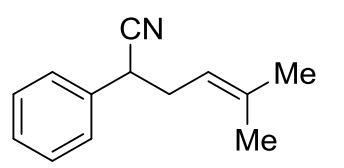

5-methyl-2-phenylhex-4-enenitrile (1ze) ${ }^{12}$ : Colorless liquid (2.45 g, $44 \%$ yield). ${ }^{1} \mathrm{H}$ NMR $\left(500 \mathrm{MHz}, \mathrm{CDCl}_{3}\right) \delta 7.42-7.29(\mathrm{~m}, 5 \mathrm{H}), 5.22-5.14(\mathrm{~m}, 1 \mathrm{H}), 3.77$ (dd, $J$ $=8.1,6.4 \mathrm{~Hz}, 1 \mathrm{H}), 2.68-2.48(\mathrm{~m}, 2 \mathrm{H}), 1.72(\mathrm{~s}, 3 \mathrm{H}), 1.54(\mathrm{~s}, 3 \mathrm{H}) ;{ }^{13} \mathrm{C}\left\{{ }^{1} \mathrm{H}\right\} \mathrm{NMR}$ $\left(125 \mathrm{MHz}, \mathrm{CDCl}_{3}\right) \delta 136.7,135.7,129.0,128.0,127.4,120.8,118.5,37.9,34.5,25.7,17.8$; HRMS (ESI-TOF) calcd for $\mathrm{C}_{13} \mathrm{H}_{16} \mathrm{~N}\left([\mathrm{M}+\mathrm{H}]^{+}\right)$186.1277, found 186.1271.

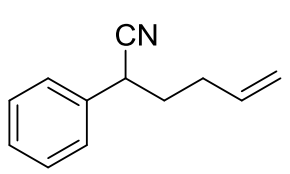

2-phenylhex-5-enenitrile (1zg) ${ }^{12}$ : Colorless liquid (2.36 g, 46\% yield). ${ }^{1} \mathrm{H}$ NMR (500 $\left.\mathrm{MHz}, \mathrm{CDCl}_{3}\right) \delta 7.45-7.30(\mathrm{~m}, 5 \mathrm{H}), 5.78(\mathrm{ddt}, J=16.9,10.2,6.7 \mathrm{~Hz}, 1 \mathrm{H}), 5.16-5.04$ $(\mathrm{m}, 2 \mathrm{H}), 3.80(\mathrm{dd}, J=8.7,6.3 \mathrm{~Hz}, 1 \mathrm{H}), 2.31-2.18(\mathrm{~m}, 2 \mathrm{H}), 2.12-2.00(\mathrm{~m}, 1 \mathrm{H}), 1.99$ $1.89(\mathrm{~m}, 1 \mathrm{H}) ;{ }^{13} \mathrm{C}\left\{{ }^{1} \mathrm{H}\right\}$ NMR $\left(125 \mathrm{MHz}, \mathrm{CDCl}_{3}\right) \delta 136.1,135.7,129.1,128.1,127.3$, 120.7, 116.7, 36.5, 34.9, 30.9; HRMS (ESI-TOF) calcd for $\mathrm{C}_{12} \mathrm{H}_{14} \mathrm{~N}\left([\mathrm{M}+\mathrm{H}]^{+}\right)$172.1121, found 172.1117.

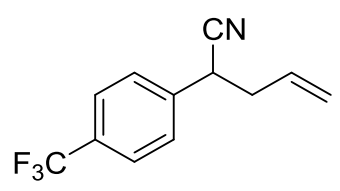

2-(4-(trifluoromethyl)phenyl)pent-4-enenitrile (1zi): Colorless liquid (2.16 g, 32\% yield). ${ }^{1} \mathrm{H}$ NMR $\left(500 \mathrm{MHz}, \mathrm{CDCl}_{3}\right) \delta 7.65(\mathrm{~d}, J=8.1 \mathrm{~Hz}, 2 \mathrm{H}), 7.47(\mathrm{~d}, J=8.1 \mathrm{~Hz}$, 2H), 5.78 (ddt, $J=17.2,10.3,7.0 \mathrm{~Hz}, 1 \mathrm{H}), 5.27-5.12$ (m, 2H), 3.94 (dd, $J=7.1$, $7.1 \mathrm{~Hz}, 1 \mathrm{H}), 2.70-2.60(\mathrm{~m}, 2 \mathrm{H}) ;{ }^{13} \mathrm{C}\left\{{ }^{1} \mathrm{H}\right\}$ NMR $\left(125 \mathrm{MHz}, \mathrm{CDCl}_{3}\right) \delta 139.1,131.8$, $130.6\left(\mathrm{q},{ }^{2} J_{\mathrm{FC}}=32.9 \mathrm{~Hz}\right), 127.9,126.1\left(\mathrm{q},{ }^{3} J_{\mathrm{FC}}=3.6 \mathrm{~Hz}\right), 123.8\left(\mathrm{q},{ }^{1} J_{\mathrm{FC}}=272.3 \mathrm{~Hz}\right), 120.0,119.5,39.6$, $37.3 ;{ }^{19} \mathrm{~F}\left\{{ }^{1} \mathrm{H}\right\}$ NMR $\left(470 \mathrm{MHz}, \mathrm{CDCl}_{3}\right) \delta-62.7$; HRMS (ESI-TOF) calcd for $\mathrm{C}_{12} \mathrm{H}_{10} \mathrm{~F}_{3} \mathrm{NNa}\left([\mathrm{M}+\mathrm{Na}]^{+}\right)$ 248.0658 , found 248.0663 .

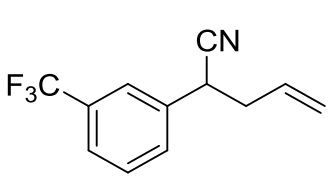

2-(3-(trifluoromethyl)phenyl)pent-4-enenitrile (1zj): Colorless liquid (2.91 g, 43\% yield). ${ }^{1} \mathrm{H}$ NMR $\left(500 \mathrm{MHz}, \mathrm{CDCl}_{3}\right) \delta 7.65-7.49(\mathrm{~m}, 4 \mathrm{H}), 5.79$ (ddt, $J=17.2,10.2$, $7.0 \mathrm{~Hz}, 1 \mathrm{H}), 5.27-5.12(\mathrm{~m}, 2 \mathrm{H}), 3.99-3.88(\mathrm{~m}, 1 \mathrm{H}), 2.74-2.58(\mathrm{~m}, 2 \mathrm{H}) ;{ }^{13} \mathrm{C}\left\{{ }^{1} \mathrm{H}\right\}$ $\operatorname{NMR}\left(125 \mathrm{MHz}, \mathrm{CDCl}_{3}\right) \delta 136.2,131.8,131.4\left(\mathrm{q},{ }^{2} J_{\mathrm{FC}}=32.6 \mathrm{~Hz}\right), 130.8,129.6$, $125.1\left(\mathrm{q},{ }^{3} J_{\mathrm{FC}}=3.6 \mathrm{~Hz}\right), 124.2\left(\mathrm{q},{ }^{3} J_{\mathrm{FC}}=3.7 \mathrm{~Hz}\right), 123.7\left(\mathrm{q},{ }^{1} J_{\mathrm{FC}}=271.0 \mathrm{~Hz}\right), 119.9,119.5,39.6,37.2$; ${ }^{19} \mathrm{~F}\left\{{ }^{1} \mathrm{H}\right\}$ NMR (470 MHz, CDCl $) \delta-62.7$; HRMS (ESI-TOF) calcd for $\mathrm{C}_{12} \mathrm{H}_{10} \mathrm{~F}_{3} \mathrm{NNa}\left([\mathrm{M}+\mathrm{Na}]^{+}\right)$ 248.0658 , found 248.0664 .

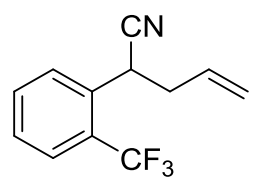

2-(2-(trifluoromethyl)phenyl)pent-4-enenitrile (1zk): Colorless liquid (3.51 g, 52\% yield). ${ }^{1} \mathrm{H}$ NMR $\left(500 \mathrm{MHz}, \mathrm{CDCl}_{3}\right) \delta 7.70(\mathrm{t}, J=6.9 \mathrm{~Hz}, 2 \mathrm{H}), 7.63(\mathrm{t}, J=7.6 \mathrm{~Hz}, 1 \mathrm{H})$, $7.46(\mathrm{t}, J=7.7 \mathrm{~Hz}, 1 \mathrm{H}), 5.93-5.79(\mathrm{~m}, 1 \mathrm{H}), 5.29-5.18(\mathrm{~m}, 2 \mathrm{H}), 4.23(\mathrm{dd}, J=8.6,5.8$ $\mathrm{Hz}, 1 \mathrm{H}), 2.70-2.55(\mathrm{~m}, 2 \mathrm{H}) ;{ }^{13} \mathrm{C}\left\{{ }^{1} \mathrm{H}\right\}$ NMR $\left(125 \mathrm{MHz}, \mathrm{CDCl}_{3}\right) \delta 134.2,132.7,132.1$, $129.7,128.4,127.7\left(\mathrm{q},{ }^{2} J_{\mathrm{FC}}=30.4 \mathrm{~Hz}\right), 126.5\left(\mathrm{q},{ }^{3} J_{\mathrm{FC}}=5.6 \mathrm{~Hz}\right), 123.9\left(\mathrm{q},{ }^{1} J_{\mathrm{FC}}=273.8 \mathrm{~Hz}\right), 120.0$, 119.9, 40.0, 34.0; ${ }^{19} \mathrm{~F}\left\{{ }^{1} \mathrm{H}\right\}$ NMR (470 MHz, $\left.\mathrm{CDCl}_{3}\right) \delta-58.9$; HRMS (ESI-TOF) calcd for $\mathrm{C}_{12} \mathrm{H}_{10} \mathrm{~F}_{3} \mathrm{NNa}$ $\left([\mathrm{M}+\mathrm{Na}]^{+}\right) 248.0658$, found 248.0653 .

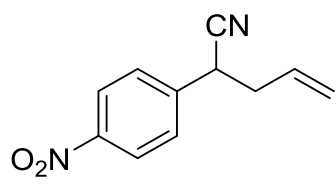

2-(4-nitrophenyl)pent-4-enenitrile (1zl): Colorless liquid (2.91 g, $48 \%$ yield). ${ }^{1} \mathrm{H}$ NMR $\left(500 \mathrm{MHz}, \mathrm{CDCl}_{3}\right) \delta 8.31-8.16(\mathrm{~m}, 2 \mathrm{H}), 7.59-7.46(\mathrm{~m}, 2 \mathrm{H}), 5.83-5.68(\mathrm{~m}$, $1 \mathrm{H}), 5.25-5.09(\mathrm{~m}, 2 \mathrm{H}), 4.02(\mathrm{dd}, J=7.0,7.0 \mathrm{~Hz}, 1 \mathrm{H}), 2.72-2.61(\mathrm{~m}, 2 \mathrm{H}) ;{ }^{13} \mathrm{C}\left\{{ }^{1} \mathrm{H}\right\}$ 
NMR $\left(125 \mathrm{MHz}, \mathrm{CDCl}_{3}\right) \delta 147.6,142.1,131.4,128.5,124.2,120.3,119.0,39.3,37.1$; HRMS (ESITOF) calcd for $\mathrm{C}_{11} \mathrm{H}_{10} \mathrm{~N}_{2} \mathrm{O}_{2} \mathrm{Na}\left([\mathrm{M}+\mathrm{Na}]^{+}\right)$225.0634, found 225.0629.

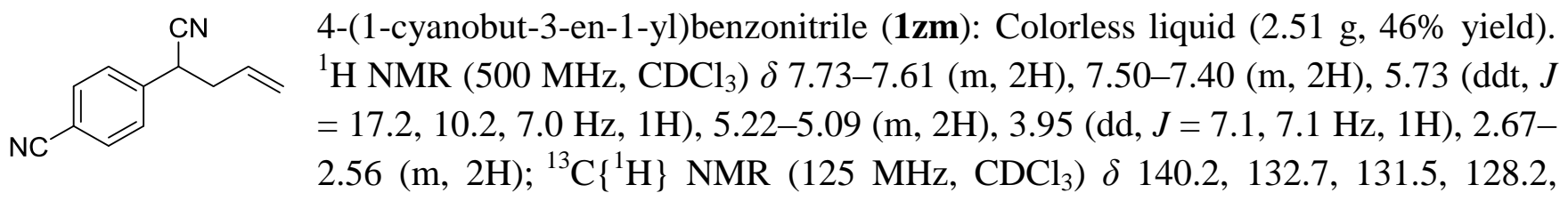
120.1, 119.1, 118.0, 112.2, 39.2, 37.2; HRMS (ESI-TOF) calcd for $\mathrm{C}_{12} \mathrm{H}_{11} \mathrm{~N}_{2}\left([\mathrm{M}+\mathrm{H}]^{+}\right)$183.0917, found 183.0925 .

\section{Characterization Data of Substrates $2 t$ and $2 u$}

${ }_{\mathrm{Ph}} \widehat{N}_{\mathrm{N}} \sim \mathbf{N}$-Benzylprop-2-en-1-amine (2t) ${ }^{14 \mathrm{a}}$ : Colorless liquid (1.27 g, 43\% yield). ${ }^{1} \mathrm{H}$ NMR $\left(500 \mathrm{MHz}, \mathrm{CDCl}_{3}\right) \delta 7.32(\mathrm{dd}, J=8.5,4.5 \mathrm{~Hz}, 4 \mathrm{H}), 7.26(\mathrm{ddd}, J=10.1,4.9,4.0 \mathrm{~Hz}$, $1 \mathrm{H}), 5.94$ (ddt, $J=17.1,10.3,6.0 \mathrm{~Hz}, 1 \mathrm{H}), 5.21(\mathrm{dq}, J=17.1,1.7 \mathrm{~Hz}, 1 \mathrm{H}), 5.15-5.08(\mathrm{~m}, 1 \mathrm{H}), 3.80$ (s, $2 \mathrm{H}), 3.29(\mathrm{dt}, J=6.0,1.5 \mathrm{~Hz}, 2 \mathrm{H}) ;{ }^{13} \mathrm{C}\left\{{ }^{1} \mathrm{H}\right\} \mathrm{NMR}\left(125 \mathrm{MHz}, \mathrm{CDCl}_{3}\right) \delta 140.3,136.8,128.4,128.2$, 127.0, 116.0, 53.3, 51.8; HRMS (ESI-TOF) calcd for $\mathrm{C}_{10} \mathrm{H}_{14} \mathrm{~N}\left([\mathrm{M}+\mathrm{H}]^{+}\right)$148.1121, found 148.1125.

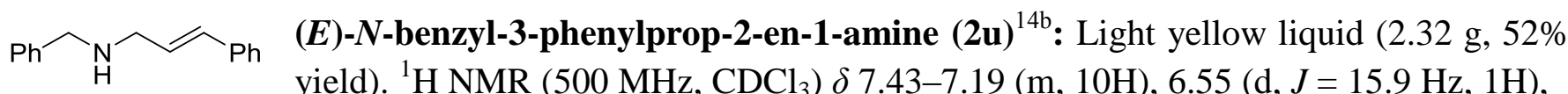
$6.33(\mathrm{dt}, J=15.9,6.3 \mathrm{~Hz}, 1 \mathrm{H}), 3.85(\mathrm{~s}, 2 \mathrm{H}), 3.45(\mathrm{dd}, J=6.3,1.4 \mathrm{~Hz}, 2 \mathrm{H}) ;{ }^{13} \mathrm{C}\left\{{ }^{1} \mathrm{H}\right\} \mathrm{NMR}(125 \mathrm{MHz}$, $\left.\mathrm{CDCl}_{3}\right) \delta 140.2,137.1,131.5,128.6,128.5,128.4,128.2,127.4,127.0,126.3,53.3,51.2$; HRMS (ESITOF) calcd for $\mathrm{C}_{16} \mathrm{H}_{18} \mathrm{~N}\left([\mathrm{M}+\mathrm{H}]^{+}\right) 224.1434$, found 224.1440 .

\section{X-ray Crystallographic Analysis Data for 3ah}

The single crystals of 3ah suitable for X-ray crystallographic analysis were obtained by recrystallization from a mixed solvent of dichloromethane and petroleum ether. The single crystal X-ray diffraction data for the compound was collected on a diffractometer with graphite monchromated Mo $\mathrm{K}_{\alpha}$ radiation $(\lambda=$ $0.71073 \AA$ ). Saint program and SADABS program carried out the data integration. The structure was solved by a direct method and refined on $\mathrm{F}^{2}$ using SHELXTL suite of program. All non-hydrogen atoms were anisotropically refined by full-matrix least squares methods. All hydrogen atoms were geometrically generated and isotropically refined using a riding model. The details of the X-ray data collection, structure solution and structure refinements are given in Table S1.

Figure S1 X-ray structure (30\% probability ellipsoids) of 3ah.

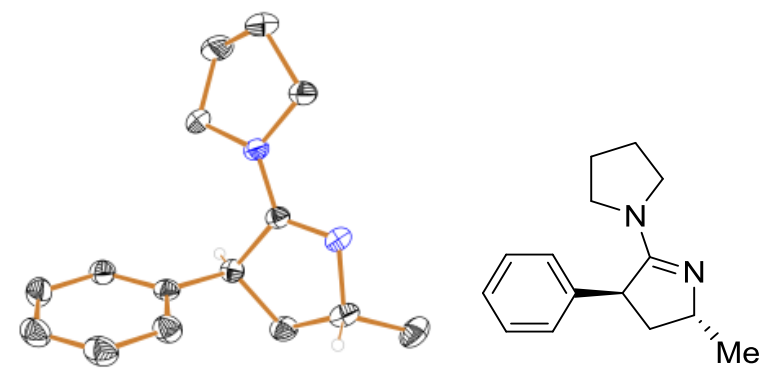

Compound 3ah 
Table S1 Crystallographic data and structural refinement details for compound 3ah.

\begin{tabular}{ll}
\hline Compound & 3ah \\
\hline Formula & $\mathrm{C}_{15} \mathrm{H}_{20} \mathrm{~N}_{2}$ \\
Formula weight & 228.33 \\
Temperature/K & 300 \\
Crystal system & Monoclinic \\
Space group & $\mathrm{P} 21 / \mathrm{n}$ \\
$a, \AA$ & $5.9791(7)$ \\
$b, \AA$ & $8.0781(9)$ \\
$c, \AA$ & $26.645(3)$ \\
$\alpha$, deg & $/$ \\
$\beta$, deg & $94.441(4)$ \\
$\gamma$, deg & $/$ \\
$V / \AA$ & $1283.1(3)$ \\
$Z$ & 1 \\
$\mu$, mm & -1 \\
$\theta$ range for data collection, deg & 0.070 \\
Reflections collected & 3.0 to 27.5 \\
Unique reflections $/ \mathrm{R}_{\text {int }}$ & 31536 \\
Goodness-of-fit on $\mathrm{F}^{2}$ & $2935 / \mathrm{R}(\mathrm{int})=0.137$ \\
$\mathrm{R}, \mathrm{R}_{\mathrm{w}}[\mathrm{I}>2 \sigma(\mathrm{I})]$ & 1.02 \\
Residual $\rho /\left(\mathrm{e} \cdot \AA^{-3}\right)$ & $0.0571,0.1620$ \\
$\mathrm{CCDC}$ & $0.26,-0.19$ \\
\hline
\end{tabular}

\section{References}

1. Sheng, E.; Wang, S.; Yang, G.; Zhou, S.; Cheng, L.; Zhang, K.; Huang, Z. A New Heteroatom Coordination Promoted Homolysis of the $\mathrm{Yb}-\mathrm{N}$ Bond. Synthesis and Structural Characterization of a New Class of Ytterbium(II) and Ytterbium(III) Complexes with Amido and Indenyl Ligands and Catalytic Activities of Ytterbium(II) Complexes. Organometallics 2003, 22, 684-692.

2. Zhou, S.-L.; Wang, S.-W.; Yang, G.-S.; Liu, X.-Y.; Sheng, E.-H.; Zhang, K.-H.; Cheng, L.; Huang, Z.-X. Synthesis, Structure, and Catalytic Activity of Tetracoordinate Lanthanide Amides $\left[\left(\mathrm{Me}_{3} \mathrm{Si}\right)_{2} \mathrm{~N}\right]_{3} \operatorname{Ln}(\mu-\mathrm{Cl}) \mathrm{Li}(\mathrm{THF})_{3}(\mathrm{Ln}=\mathrm{Nd}, \mathrm{Sm}, \mathrm{Eu})$. Polyhedron 2003, 22, 1019-1024.

3. Xie, M.-H.; Liu, X.-Y.; Wang, S.-W.; Liu, L.; Wu, Y.-Y.; Yang, G.-S.; Zhou, S.-L.; Sheng, E.-H.; Huang, Z.-X. Synthesis, Structure and Catalytic Activity Comparison of Tris- and Tetracoordinated Lanthanide Amides. Chin. J. Chem. 2010, 22, 678-682.

4. Bradley, D. C.; Ghotra, J. S.; Alan Hart, F. Low Co-ordination Numbers in Lanthanide and Actinide Compounds. Part 1. The Preparation and Characterization of Tris\{ bis(trimethylsilyl)amido)lanthanides. J. Chem. Soc. Dalton Trans. 1973, 1021-1023.

5. Ling, J.; Peng, H.; Shen, Z. Q. Deprotonation Reaction of $\alpha$-amino Acid $N$-carboxyanhydride at 4CH Position by Yttrium Tris[bis(trimethylsilyl)amide]. J. Polym. Sci. Part A: Polym. Chem. 2012, 50, 3743-3749.

6. Arndt, S.; Voth, P.; Spaniol, T. P.; Okuda, J. Dimeric Hydride Complexes of Rare-Earth Metals Containing a Linked Amido-Cyclopentadienyl Ligand: Synthesis, Characterization, and Monomer- 
Dimer Equilibrium. Organometallics 2000, 19, 4690-4700.

7. Xie, H.; Wu, C.; Cui, D.; Wang, Y. Ligand-Free Scandium Alkyl and Alkoxide Complexes for Immortal Ring-Opening Polymerization of Lactide. J. Organomet. Chem. 2018, 875, 5-10.

8. Wayda, A. L.; Atwood, J. L.; Hunter, W. E. Homoleptic Organolanthanoid Hydrocarbyls. The Synthesis and X-ray Crystal Structure of Trls[o-((dimethylamlno)methyl)phenyl]lutetium. Organometallics 1984, 3, 939-941.

9. Harder, S. Syntheses and Structures of Homoleptic Lanthanide Complexes with Chelating $o$ Dimethylaminobenzyl Ligands: Key Precursors in Lanthanide Chemistry. Organometallics 2005, 24, 373-379.

10. (a) Manzer, L. E. Paramagnetic Organometallic Compounds of the Early Transition Metals Stabilized by Chelating Benzyl and Phenyl Ligands. J. Am. Chem. Soc. 1978, 100, 8068-8073; (b) Zhang, W. X.; Nishiura, M.; Mashiko, T.; Hou, Z. M., Half-Sandwich $o-N, N$-Dimethylaminobenzyl Complexes over the Full Size Range of Group 3 and Lanthanide Metals. Synthesis, Structural Characterization, and Catalysis of Phosphine P-H Bond Addition to Carbodiimides. Chem.-Eur. J. 2008, 14, 2167-2179.

11. Theodorou, A.; Kokotos, C. G. Green Organocatalytic Synthesis of Indolines and Pyrrolidines from Alkenes. Adv. Synth. Catal. 2017, 359, 1577-1581.

12. Horn, A.; Dussault, P. H. Synthesis of $\alpha$-Cyano and $\alpha$-Sulfonyl Cyclic Ethers via Intramolecular Reactions of Peroxides with Sulfone- and Nitrile-Stabilized Carbanions. J. Org. Chem. 2019, 84, 14611-14626.

13. Le, H.; Kyne, R. E.; Brozek, L. A.; Morken, J. P. Catalytic Enantioselective Allyl-Allyl CrossCoupling with a Borylated Allylboronate. Org. Lett. 2013, 15, 1432-1435.

14. (a) Harvey, D. F.; Sigano, D. M. Synthesis of Cyclopropylpyrrolidines via Reaction of $N-A$ llyl- $N-$ propargylamides with a Molybdenum Carbene Complex. Effect of Substituents and Reaction Conditions. J. Org. Chem. 1996, 61, 2268-2272; (b) Valant, C.; Maillet, E.; Bourguignon, J.; Bucher, B.; Utard, V.; Galzi, J.; Hibert, M. Allosteric Functional Switch of Neurokinin A-Mediated Signaling at the Neurokinin NK2 Receptor: Structural Exploration. J. Med. Chem. 2009, 52, 5999-6011.

15. Wang, M.-X.; Zhao, S.-M. Synthesis of enantiomerically enriched $(S)-(+)$-2-aryl-4-pentenoic acids and $(R)-(-)$-2-aryl-4-pentenamides via microbial hydrolysis of nitriles, a chemoenzymatic approach to stereoisomers of $\alpha, \gamma$-disubstituted $\gamma$-butyrolactones. Tetrahedron: Asymmetry 2002, 13, 1695-1702.

16. Yang, H.; Carter, R. G. Organocatalyzed, enantioselective synthesis of bicyclo-[2.2.2]-octanes containing benzylic, all-carbon quaternary centers. Tetrahedron 2010, 66, 4854-4859.

17. Wang, D.; Zhu, N.; Chen, P.; Lin, Z.; Liu, G. Enantioselective Decarboxylative Cyanation Employing Cooperative Photoredox Catalysis and Copper Catalysis. J. Am. Chem. Soc. 2017, 139, $15632-15635$.

18. Maji, T.; Tunge, J. A. Catalytic $\alpha$-monoallylation of aryl acetonitriles. Org. Lett. 2014, 16, 5072-5075.

19. Mouhsine, B.; Karim, A.; Dumont, C.; Suisse, I.; Sauthier, M. Efficient and Clean Nickel Catalyzed $\alpha$-Allylation Reaction of Nitriles. Adv. Synth. Catal. 2021, 363, 1457-1462.

20. Roman, M.; Cannizzo, C.; Pinault, T.; Isare, B.; Andrioletti, B.; van der Schoot, P.; Bouteiller, L. Supramolecular Balance: Using Cooperativity to Amplify Weak Interactions. J. Am. Chem. Soc. 2010, 132, 16818-16824. 
21. Gao, M.; Wang, D.-X.; Zheng, Q.-Y.; Huang, Z.-T.; Wang, M.-X. Remarkable Electronic and Steric Effects in the Nitrile Biotransformations for the Preparation of Enantiopure Functionalized Carboxylic Acids and Amides: Implication for an Unsaturated Carbon-Carbon Bond Binding Domain of the Amidase. J. Org. Chem. 2007, 72, 6060-6066.

22. Zhang, J.; Wang, X.; Liu, Y.; Wang, X.; He, W. Palladium-catalyzed highly selective intramolecular bromoamination of alkenes: Efficient synthesis of substituted pyrrolidines. Appl. Organomet. Chem. 2017, 31, e3631.

23. Liu, Z.; Hartwig, J. F. Mild, Rhodium-Catalyzed Intramolecular Hydroamination of Unactivated Terminal and Internal Alkenes with Primary and Secondary Amines. J. Am. Chem. Soc. 2008, 130, $1570-1571$.

24. Nguyen, T. O.; Man, B. Y. W.; Hodgson, R.; Messerle, B. A., Intramolecular Hydroamination of Aminoalkenes using Rhodium(I) and Iridium(I) Complexes with $N, N$ - and $P, N$-Donor Ligands. Aust. J. Chem. 2011, 64, 741-746.

25. Sanjaya, S.; Chiba, S. PhI(OAc) $)_{2}$-mediated iminobromination for synthesis of bromomethyl cyclic imines starting from alkenyl carbonitriles and Grignard reagents. Tetrahedron 2011, 67, 590-596. 


\section{Copies of NMR Spectra for 1}

\section{Compound 1a}
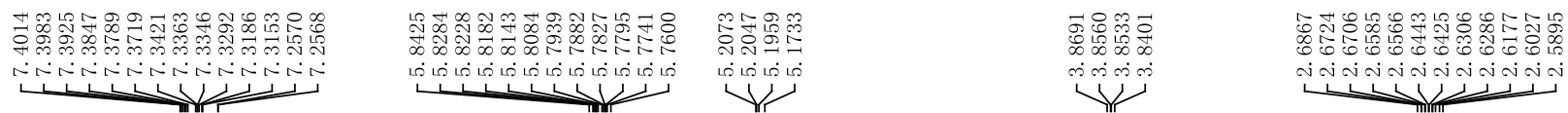

${ }^{1} \mathrm{H}$ NMR

$500 \mathrm{MHz}, \mathrm{CDCl}_{3}$
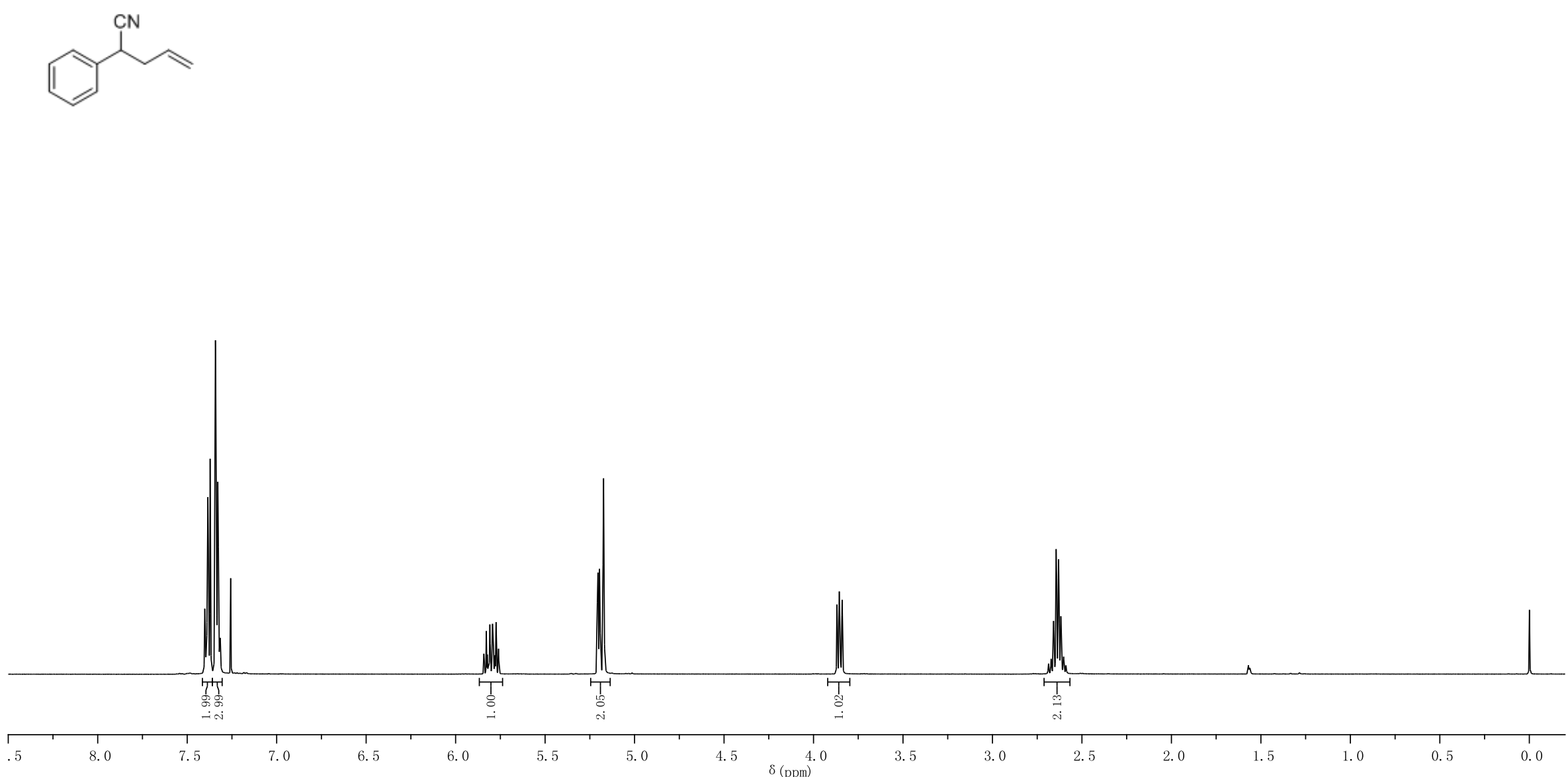


\section{Compound 1a}

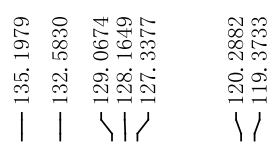

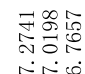

${ }^{13} \mathrm{C}\left\{{ }^{1} \mathrm{H}\right\}$ NMR

$125 \mathrm{MHz}, \mathrm{CDCl}_{3}$
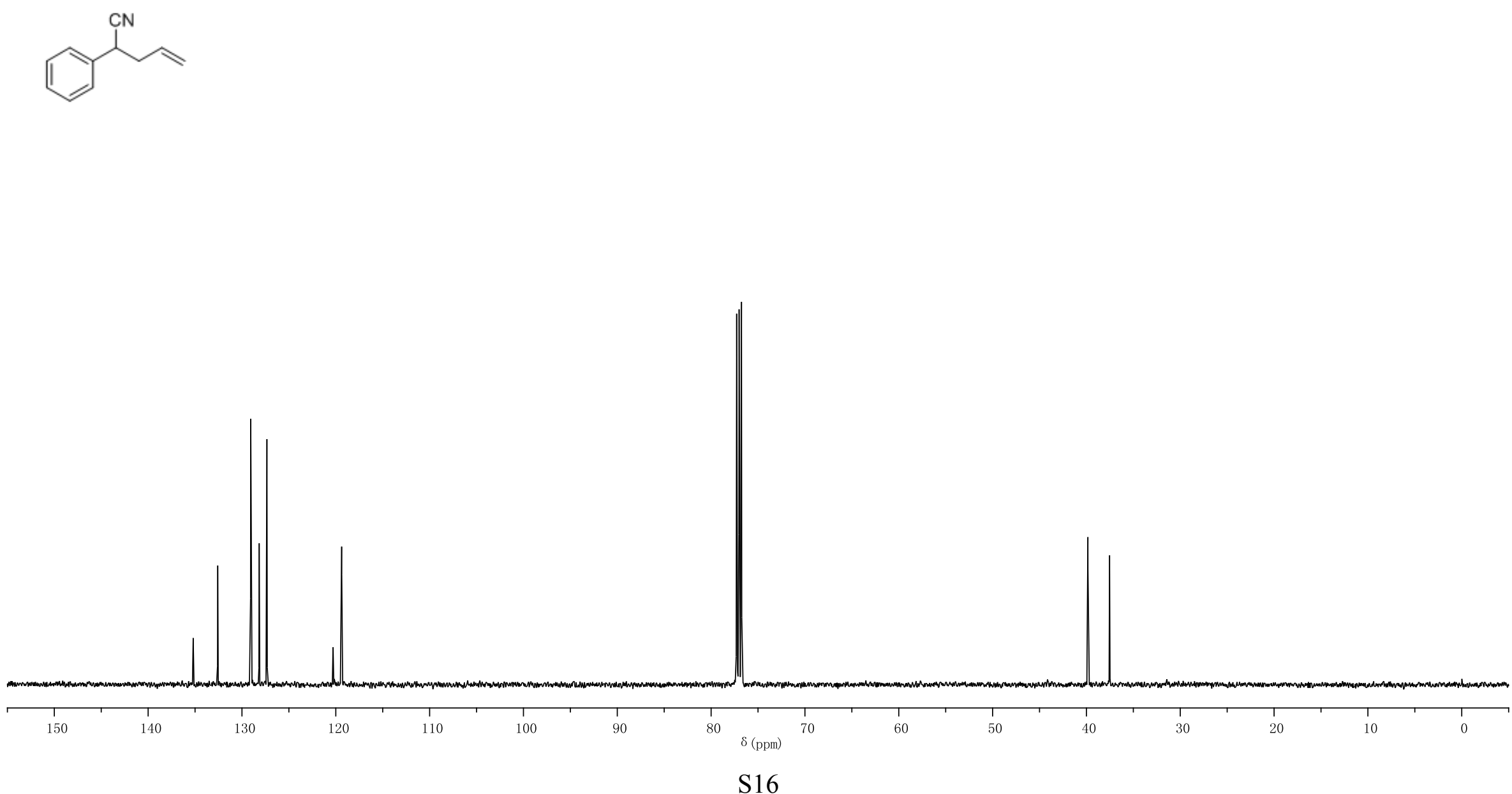


\section{Compound 1b}

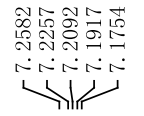

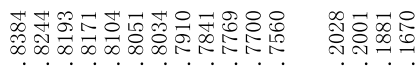
ம่

${ }^{1} \mathrm{H}$ NMR

$500 \mathrm{MHz}, \mathrm{CDCl}_{3}$
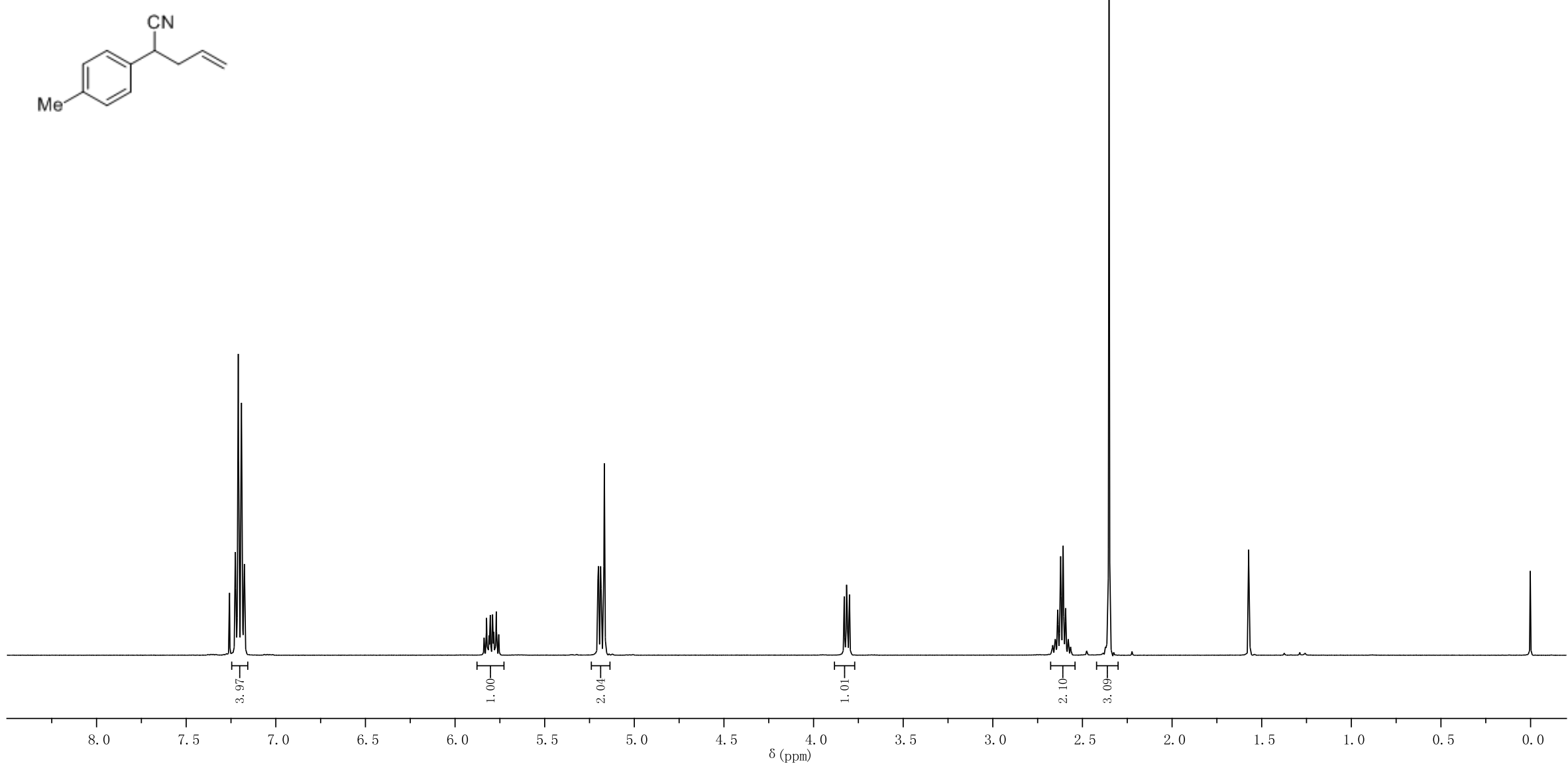


\section{Compound 1b}

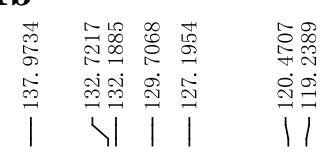
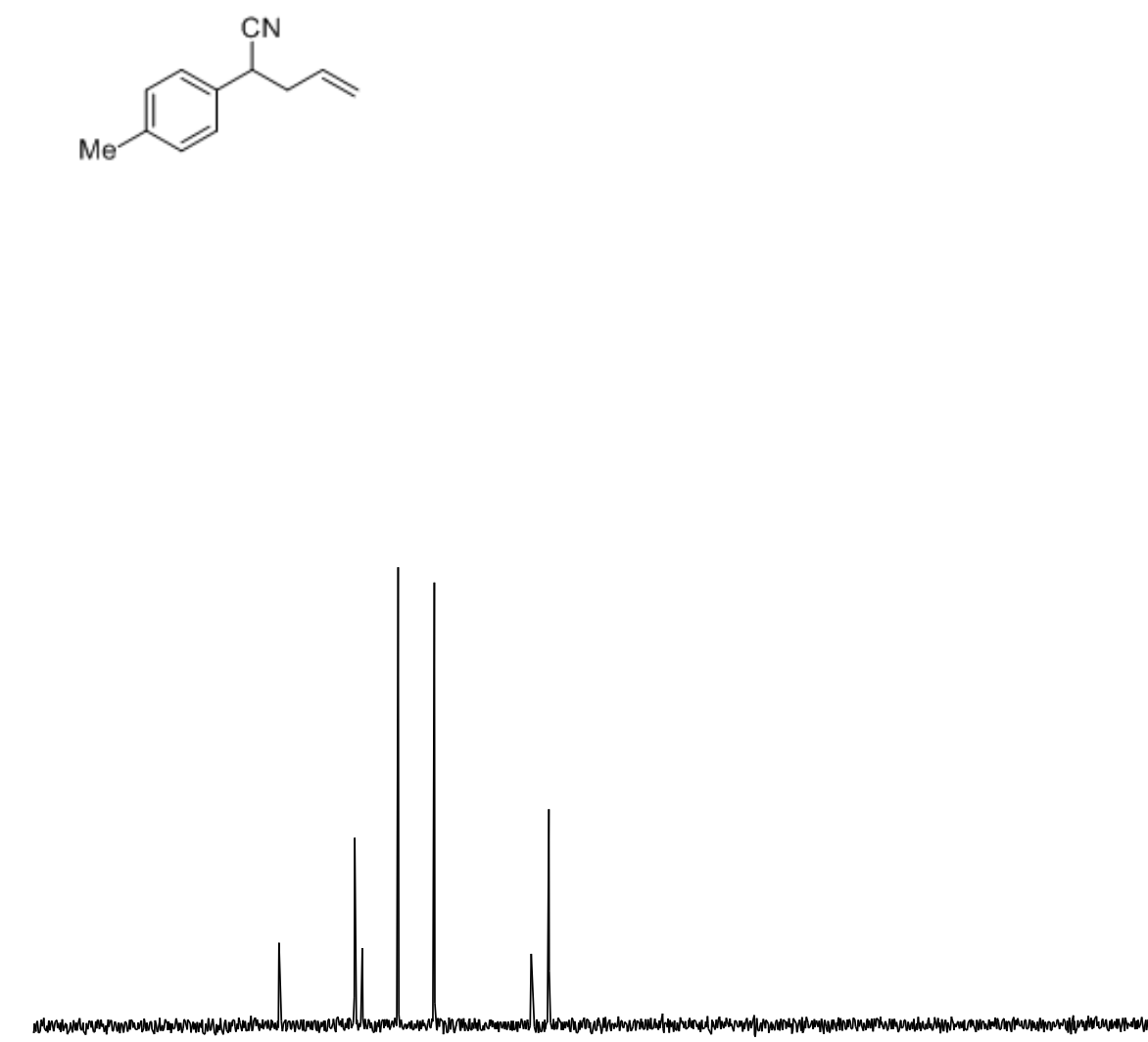

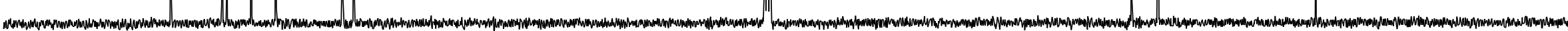

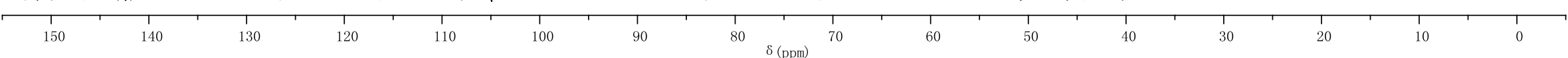




\section{Compound 1c}

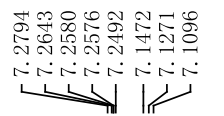

${ }^{1} \mathrm{H}$ NMR

$500 \mathrm{MHz}, \mathrm{CDCl}_{3}$<smiles>C=CCC(C#N)c1cccc(C)c1</smiles>

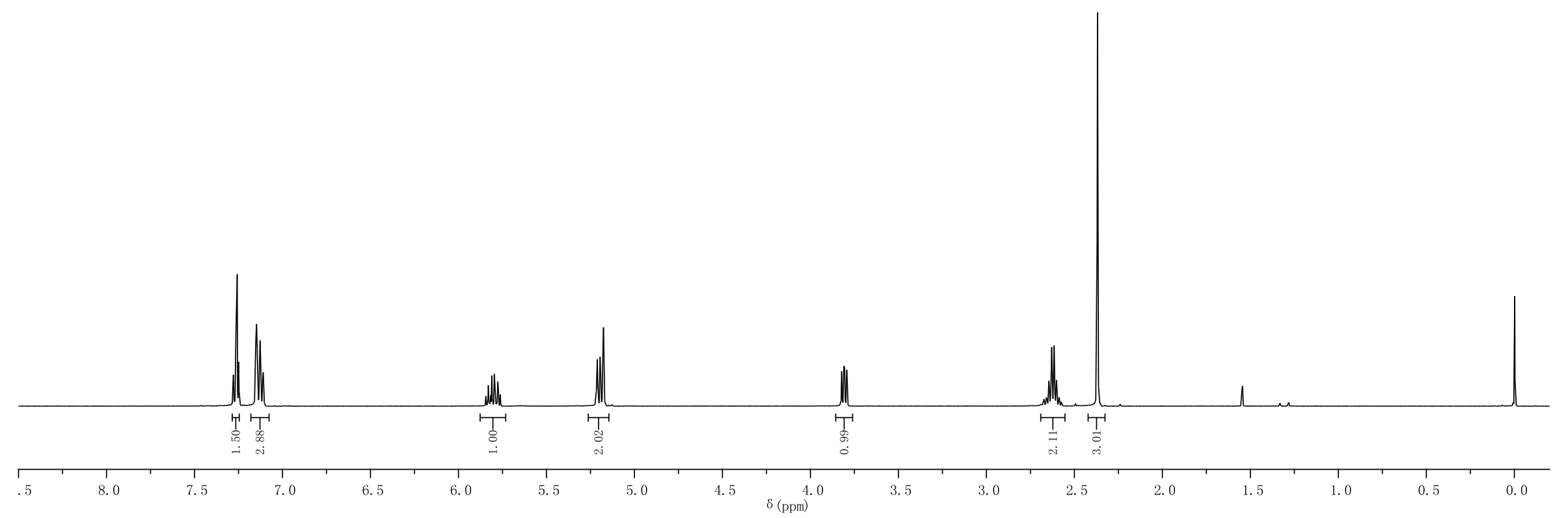




\section{Compound 1c}

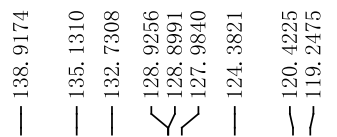

${ }^{13} \mathrm{C}\left\{{ }^{1} \mathrm{H}\right\}$ NMR

$125 \mathrm{MHz}, \mathrm{CDCl}_{3}$
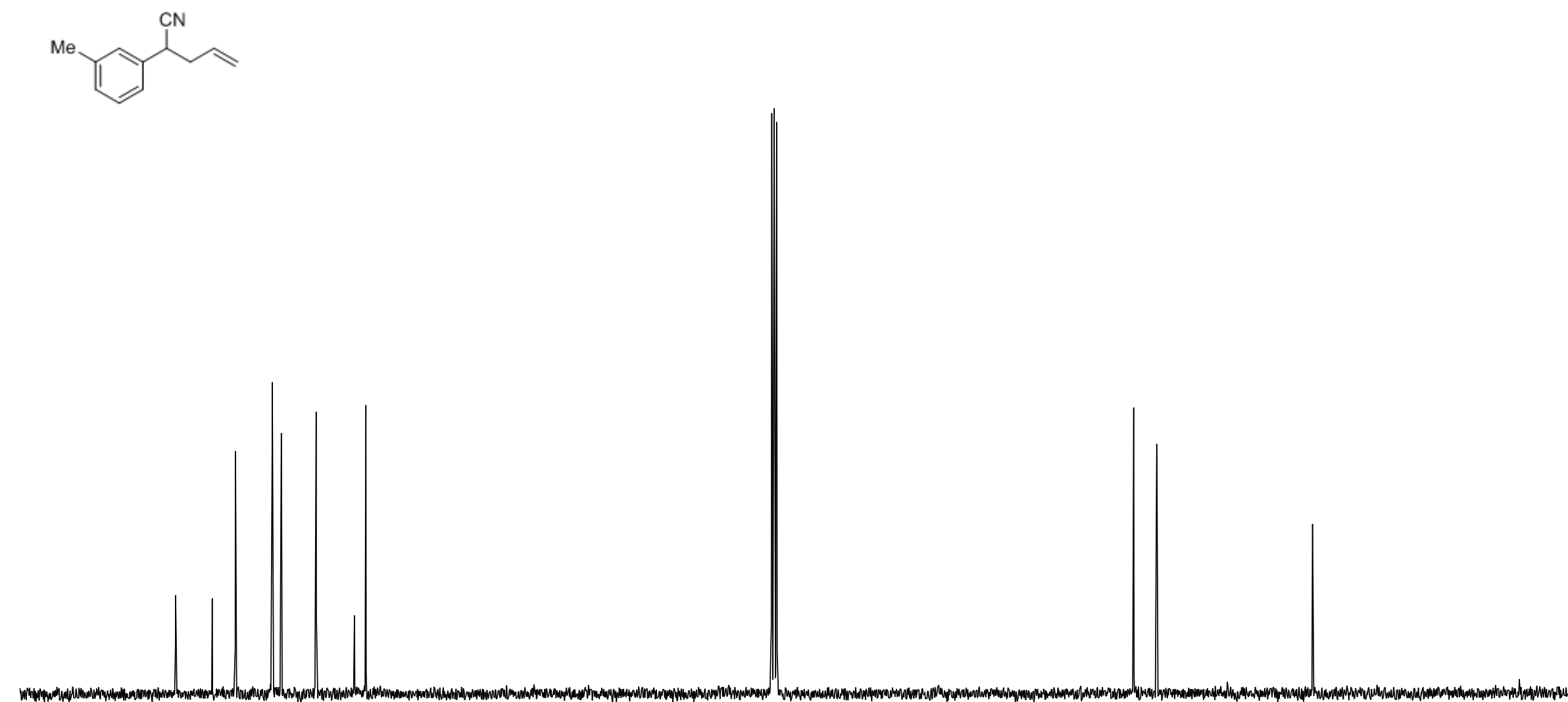


\section{Compound 1d}

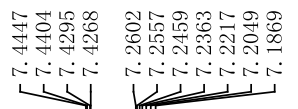

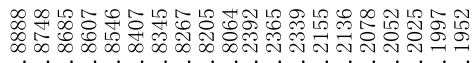

6.

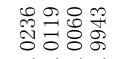

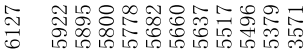

${ }^{1} \mathrm{H}$ NMR

$500 \mathrm{MHz}, \mathrm{CDCl}_{3}$
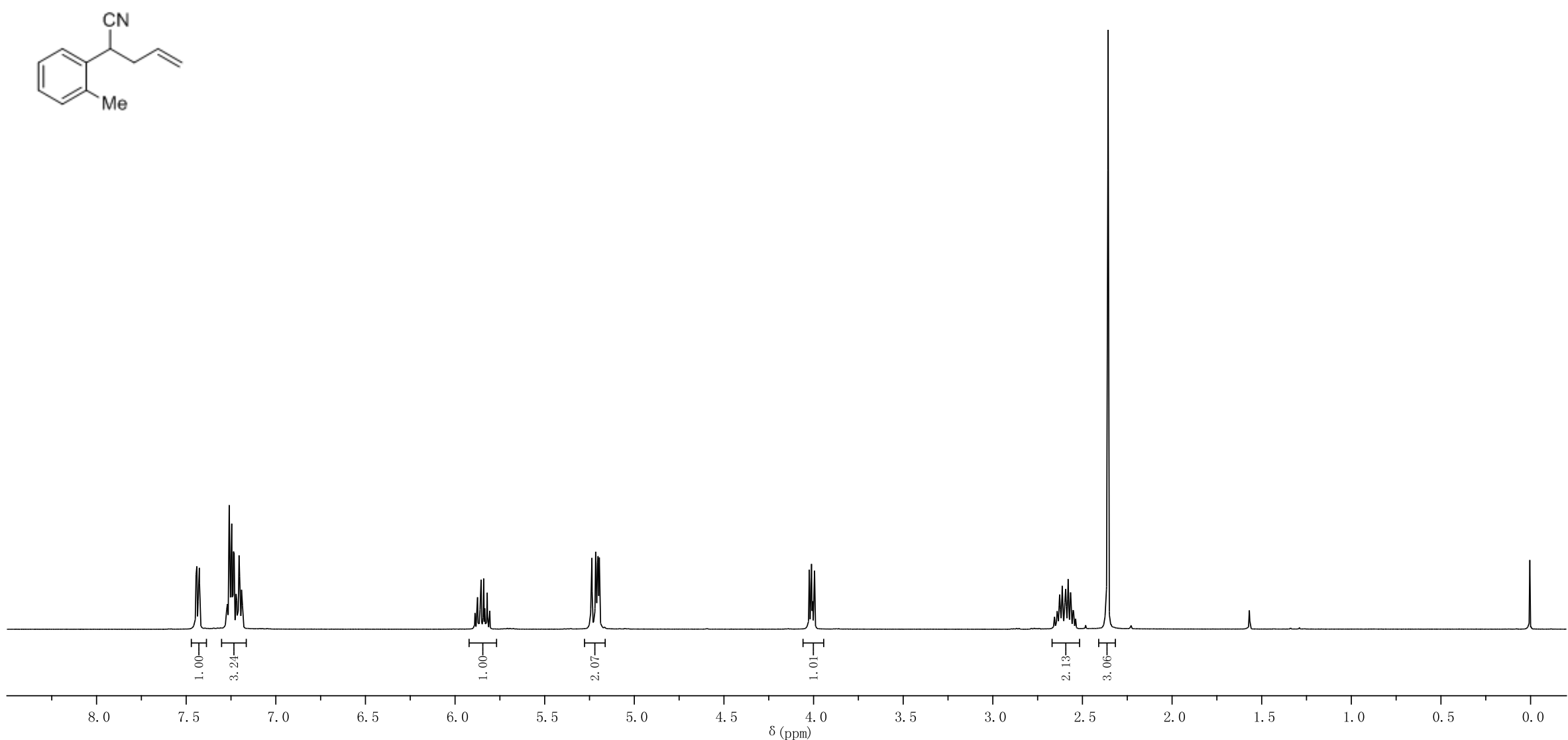


\section{Compound 1d}

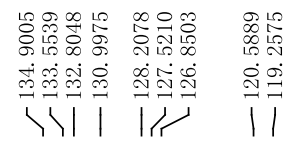

${ }^{13} \mathrm{C}\left\{{ }^{1} \mathrm{H}\right\}$ NMR

$125 \mathrm{MHz}, \mathrm{CDCl}_{3}$
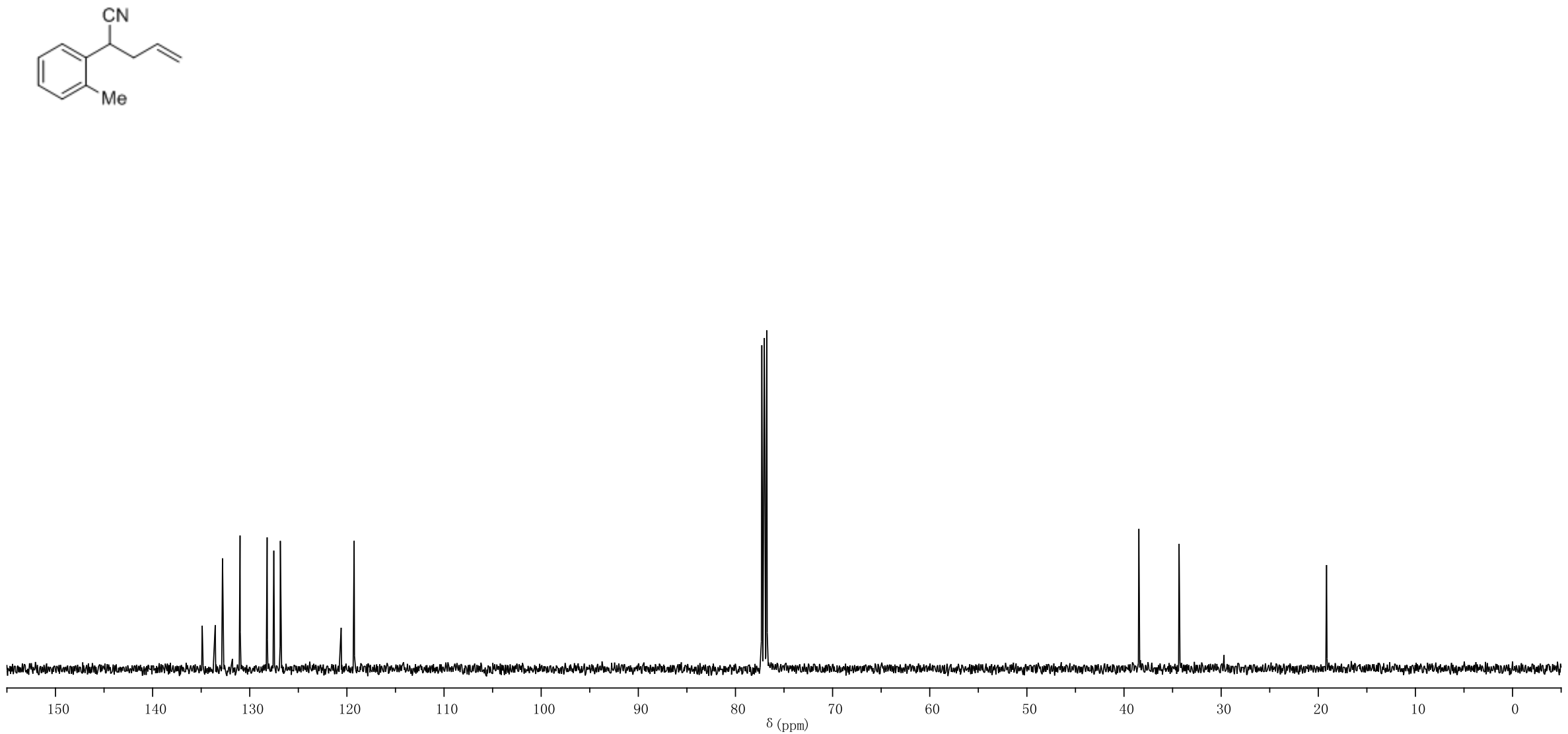


\section{Compound 1e}

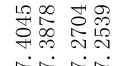

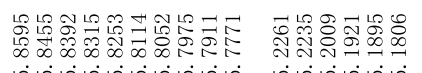

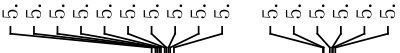

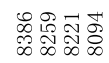

in

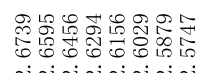

先

${ }^{1} \mathrm{H}$ NMR

$500 \mathrm{MHz}, \mathrm{CDCl}_{3}$<smiles>CC#CC(CCC)c1ccc(C)cc1</smiles>

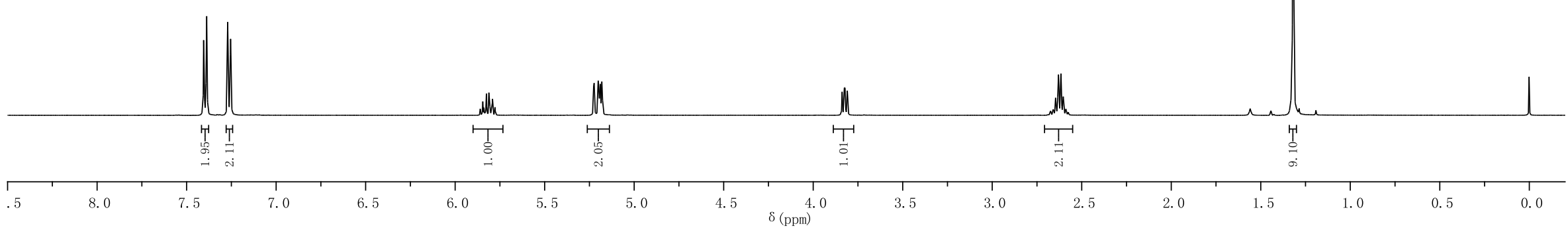




\section{Compound 1e}

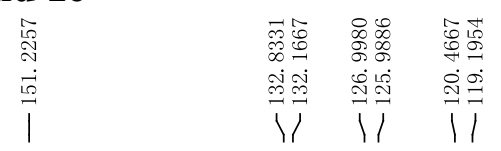

옥총

ㄴํㄴ

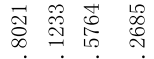

ब品䡆

| | | |

${ }^{13} \mathrm{C}\left\{{ }^{1} \mathrm{H}\right\}$ NMR

$125 \mathrm{MHz}, \mathrm{CDCl}_{3}$
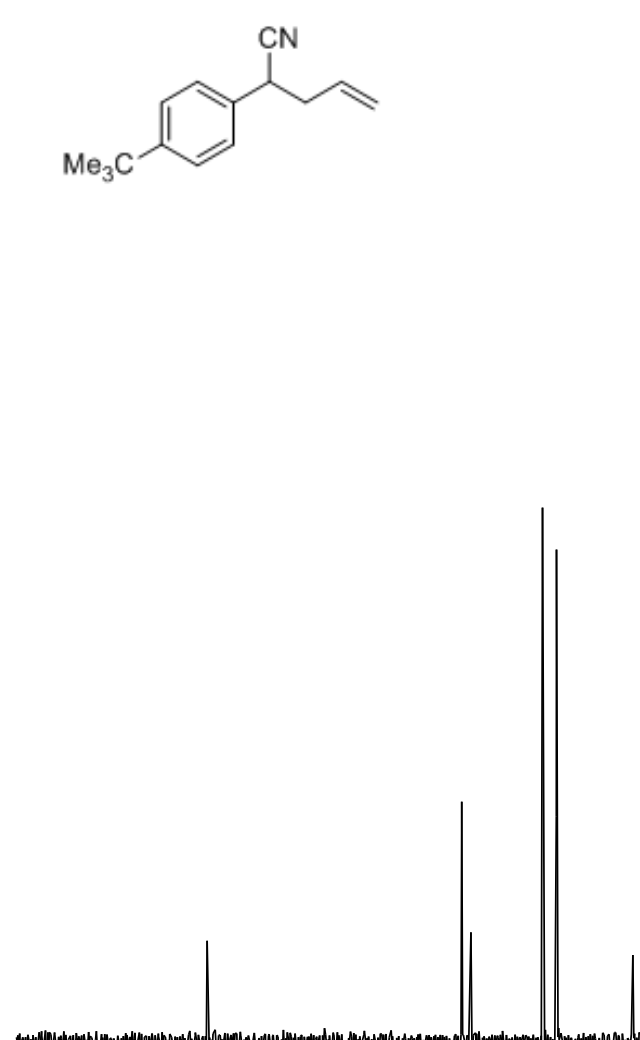

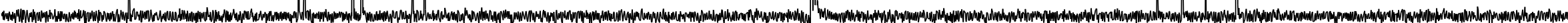


Compound 1f
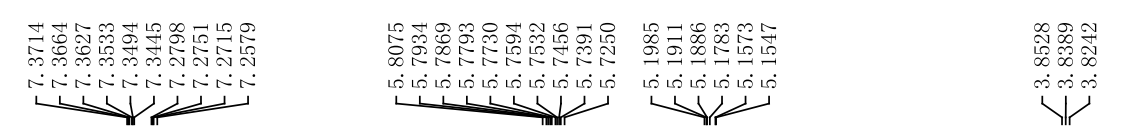

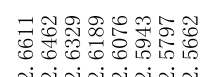

$\underbrace{\text { ن ivivivin }}$

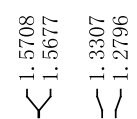

HNMR

$500 \mathrm{MHz}, \mathrm{CDCl}_{3}$
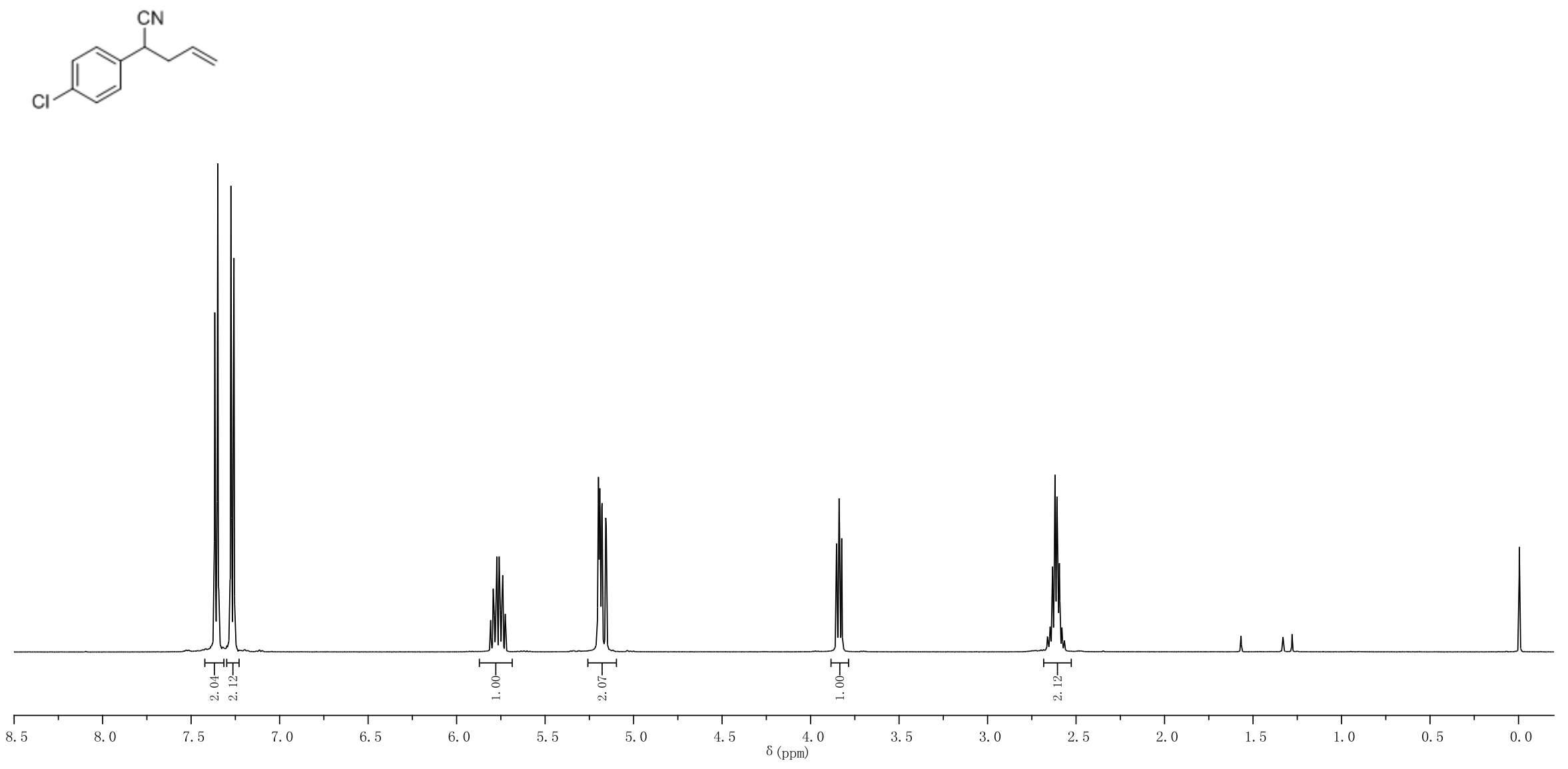


\section{Compound 1f}

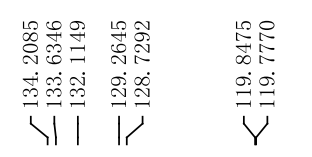

${ }^{13} \mathrm{C}\left\{{ }^{1} \mathrm{H}\right\}$ NMR

$125 \mathrm{MHz}, \mathrm{CDCl}_{3}$
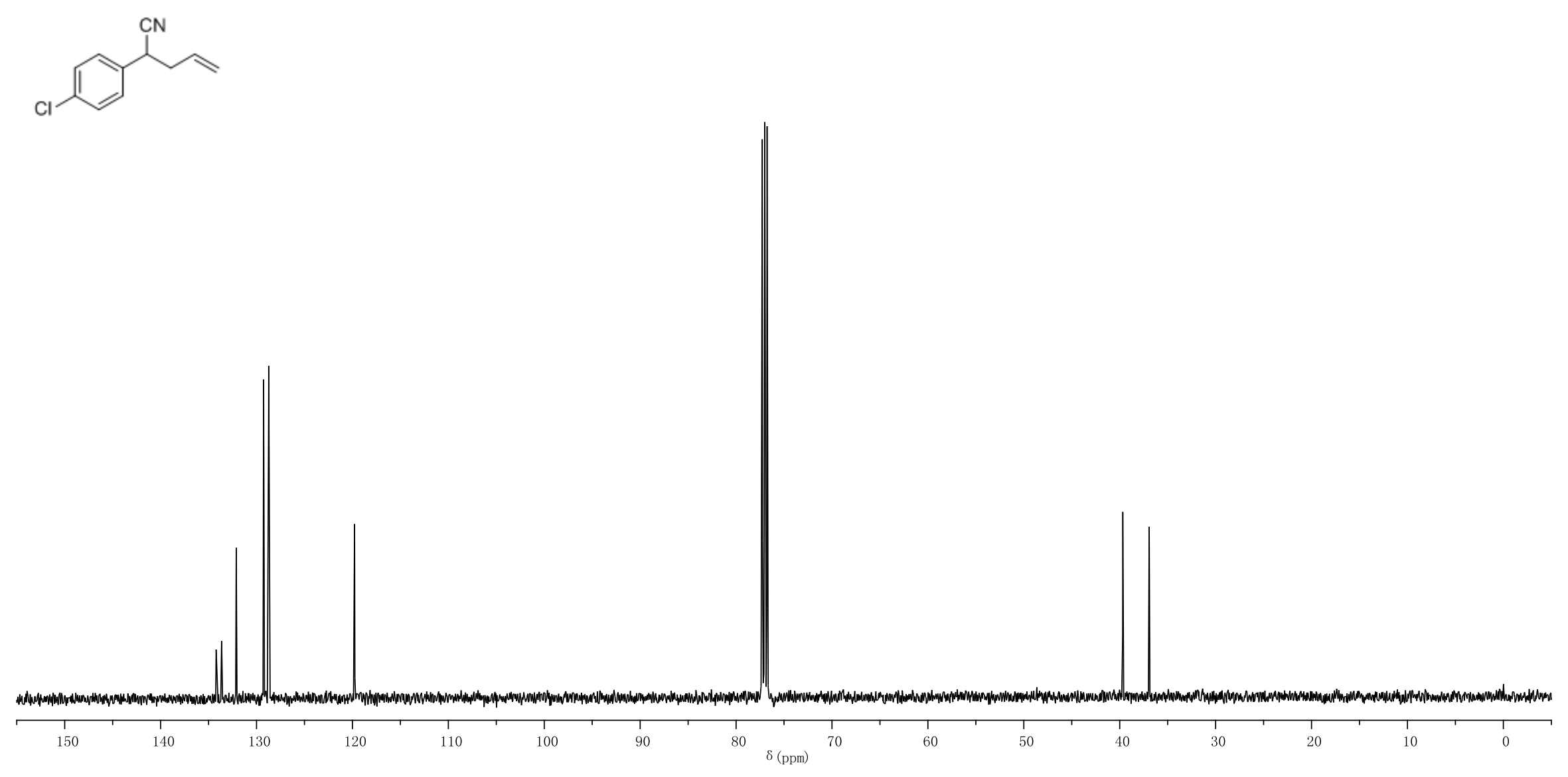


\section{Compound 1g}

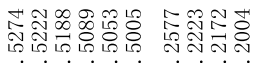

vinisinin

${ }^{1} \mathrm{H}$ NMR

$500 \mathrm{MHz}, \mathrm{CDCl}_{3}$<smiles>C=CCC(C#N)c1ccc(Br)cc1</smiles>

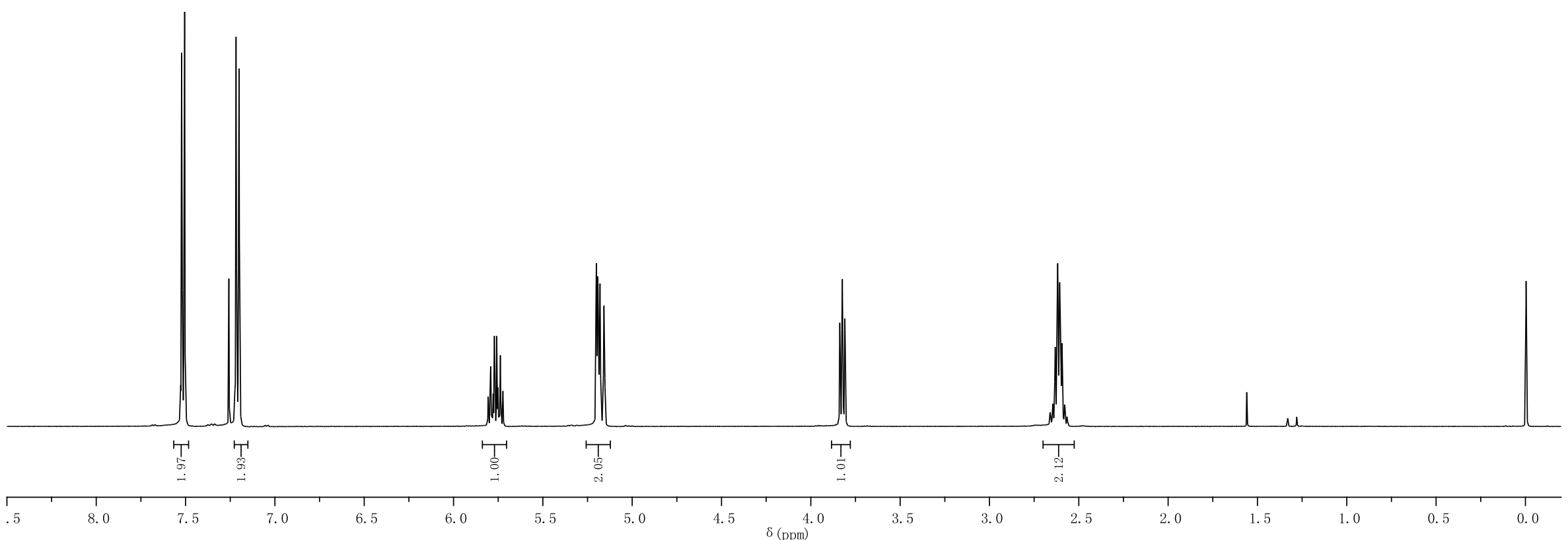


Compound lg

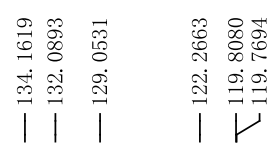

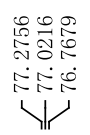

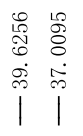

${ }^{13} \mathrm{C}\left\{{ }^{1} \mathrm{H}\right\}$ NMR

$125 \mathrm{MHz}, \mathrm{CDCl}_{3}$<smiles>C=CCC(C#N)c1ccc(Br)cc1</smiles>

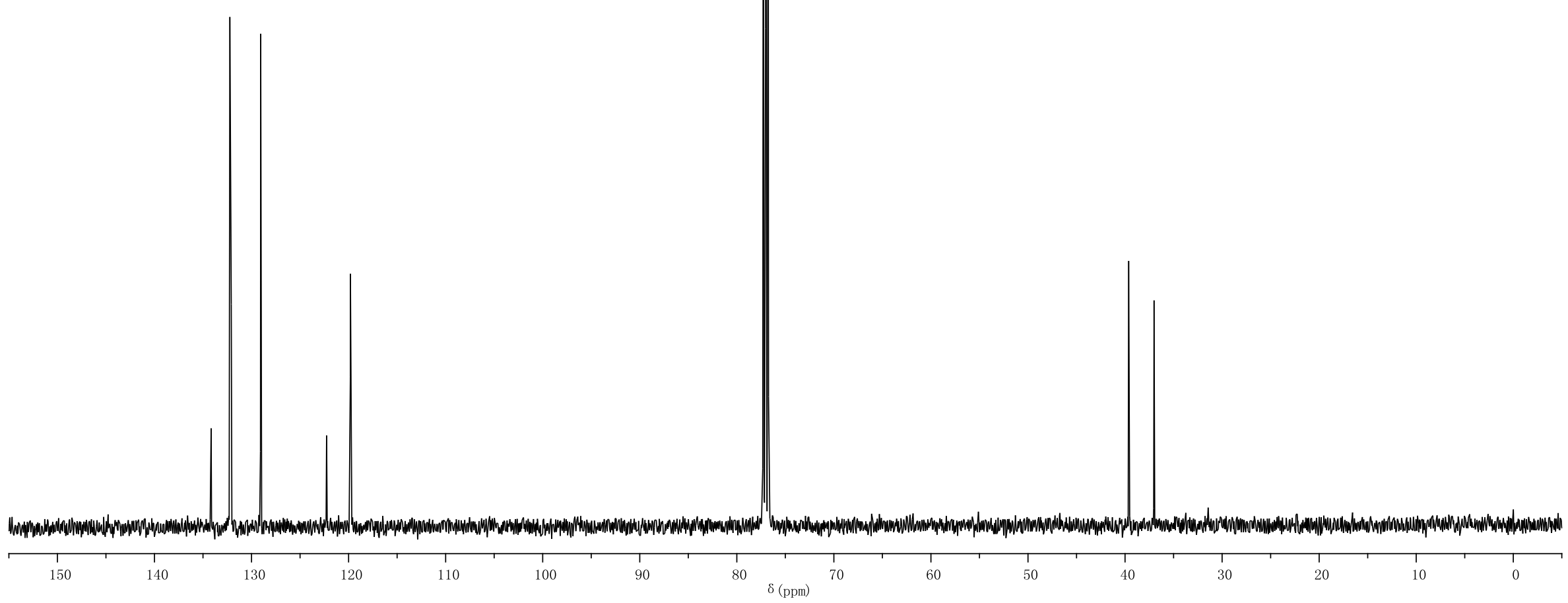

S28 


\section{Compound 1h}

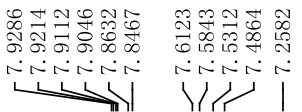

${ }^{1} \mathrm{H}$ NMR

$500 \mathrm{MHz}, \mathrm{CDCl}_{3}$
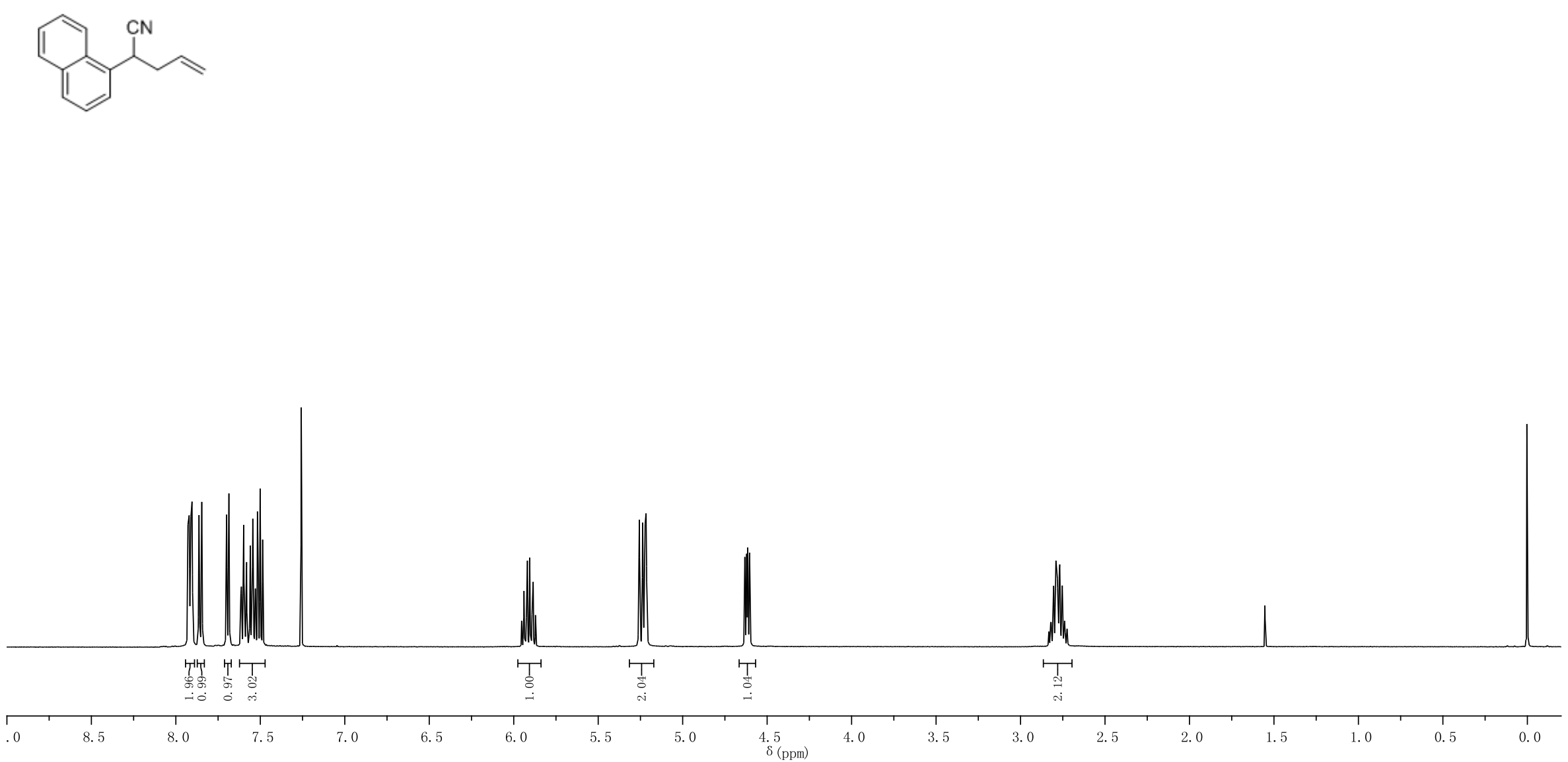
Compound 1h

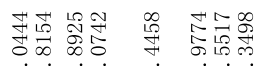

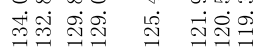

$$
\begin{aligned}
& 1111111
\end{aligned}
$$

${ }^{13} \mathrm{C}\left\{{ }^{1} \mathrm{H}\right\}$ NMR

$125 \mathrm{MHz}, \mathrm{CDCl}_{3}$
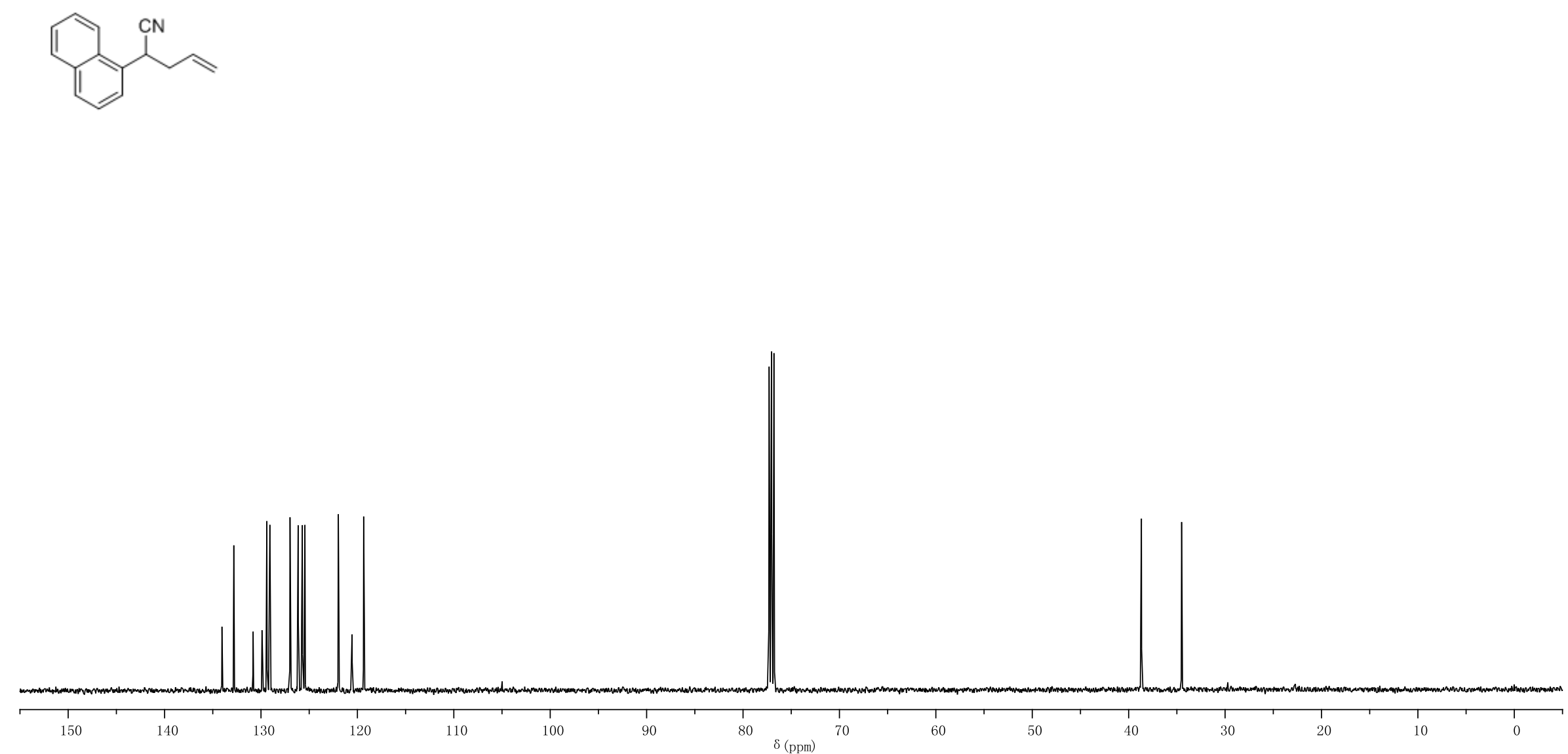


\section{Compound 1i}

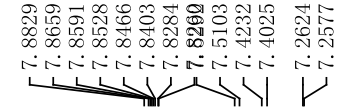

${ }^{1} \mathrm{H}$ NMR

$500 \mathrm{MHz}, \mathrm{CDCl}_{3}$
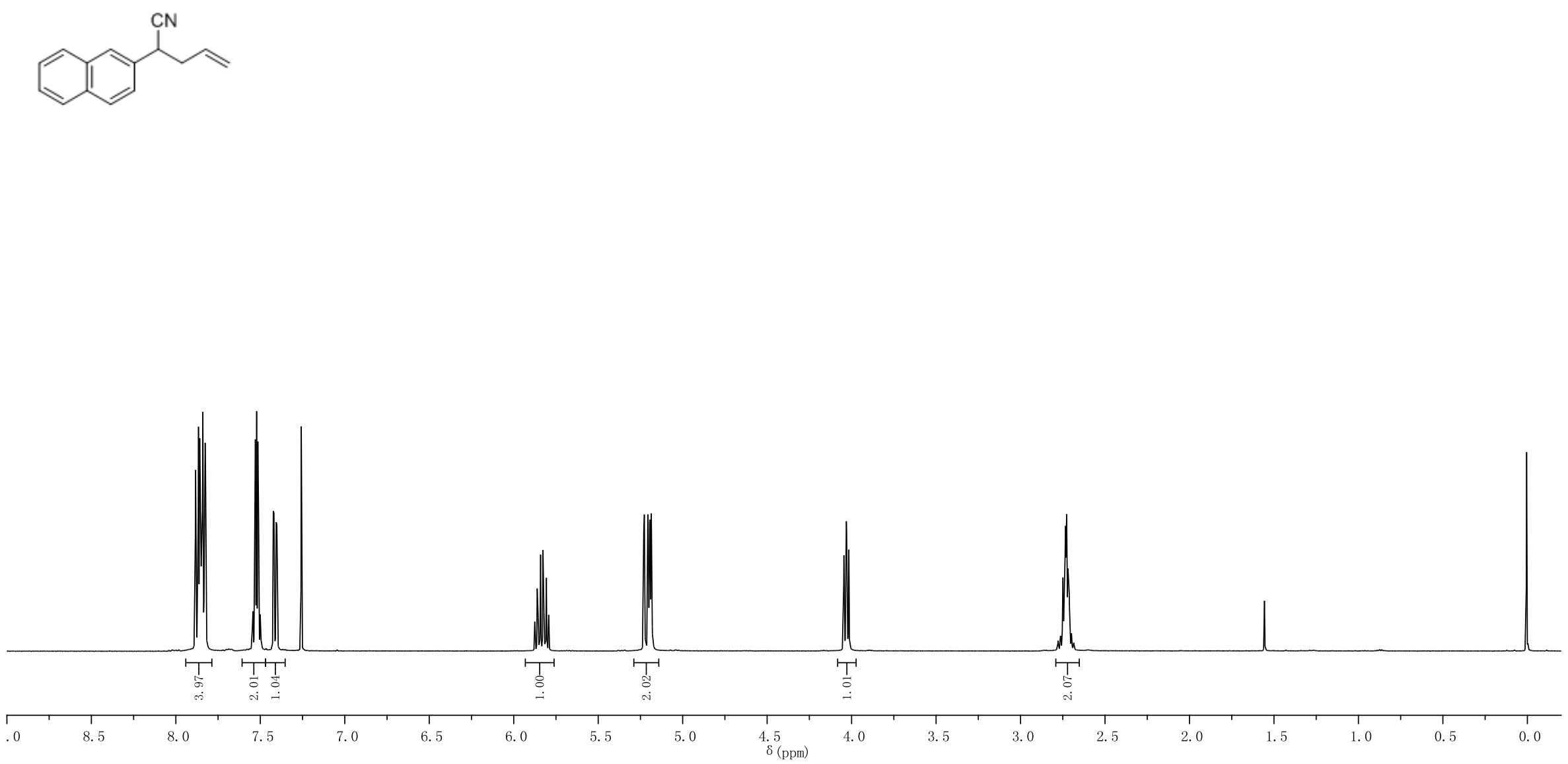
Compound 1i

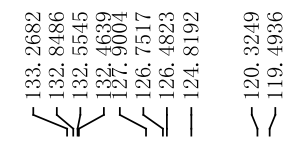

${ }^{13} \mathrm{C}\left\{{ }^{1} \mathrm{H}\right\}$ NMR

$125 \mathrm{MHz}, \mathrm{CDCl}_{3}$
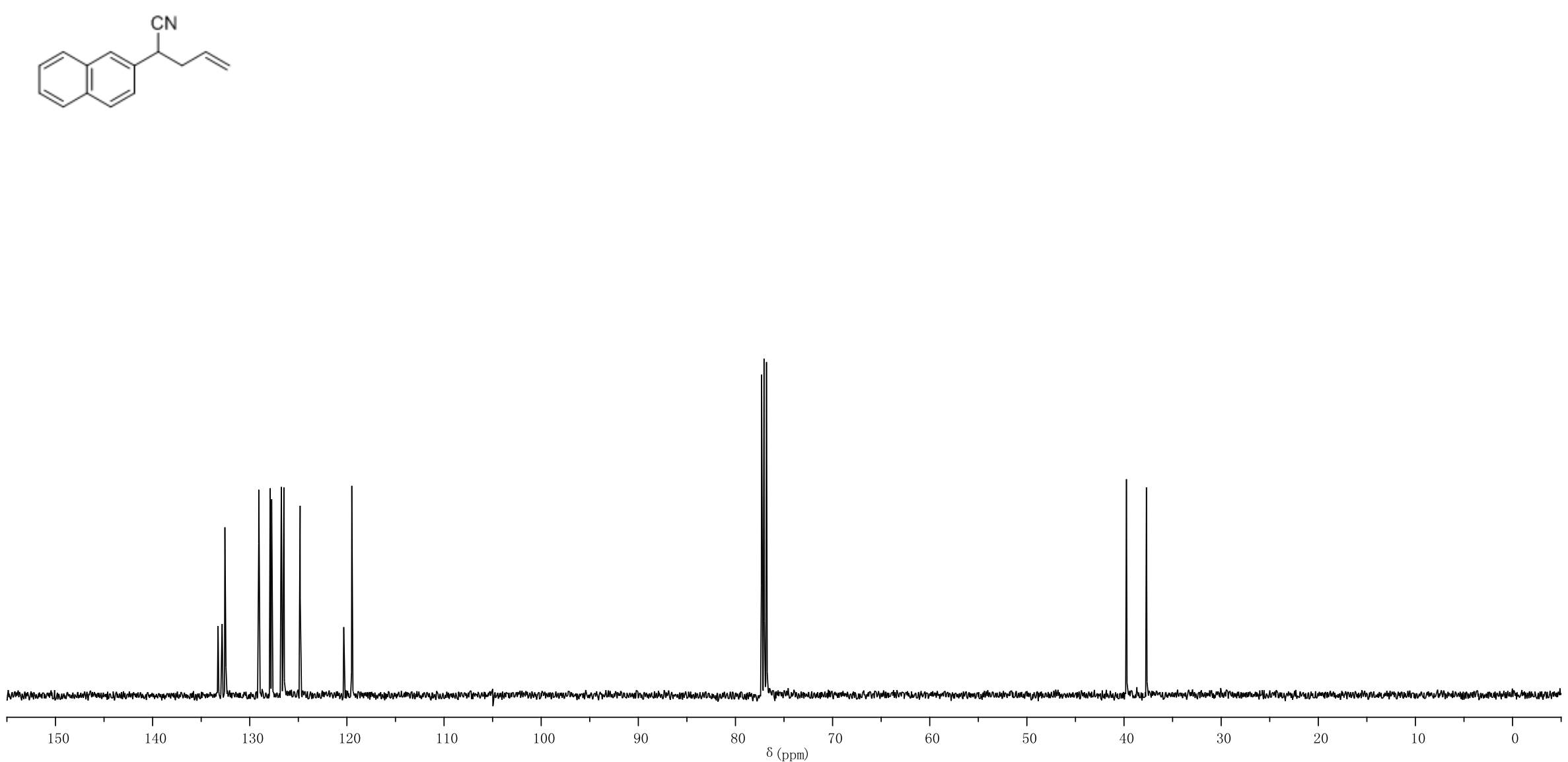


\section{Compound 1j}

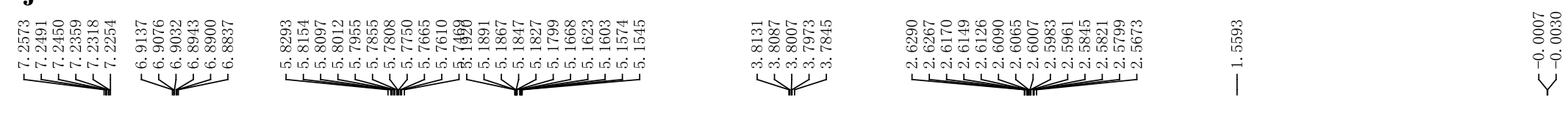

${ }^{1} \mathrm{H}$ NMR

$500 \mathrm{MHz}, \mathrm{CDCl}_{3}$
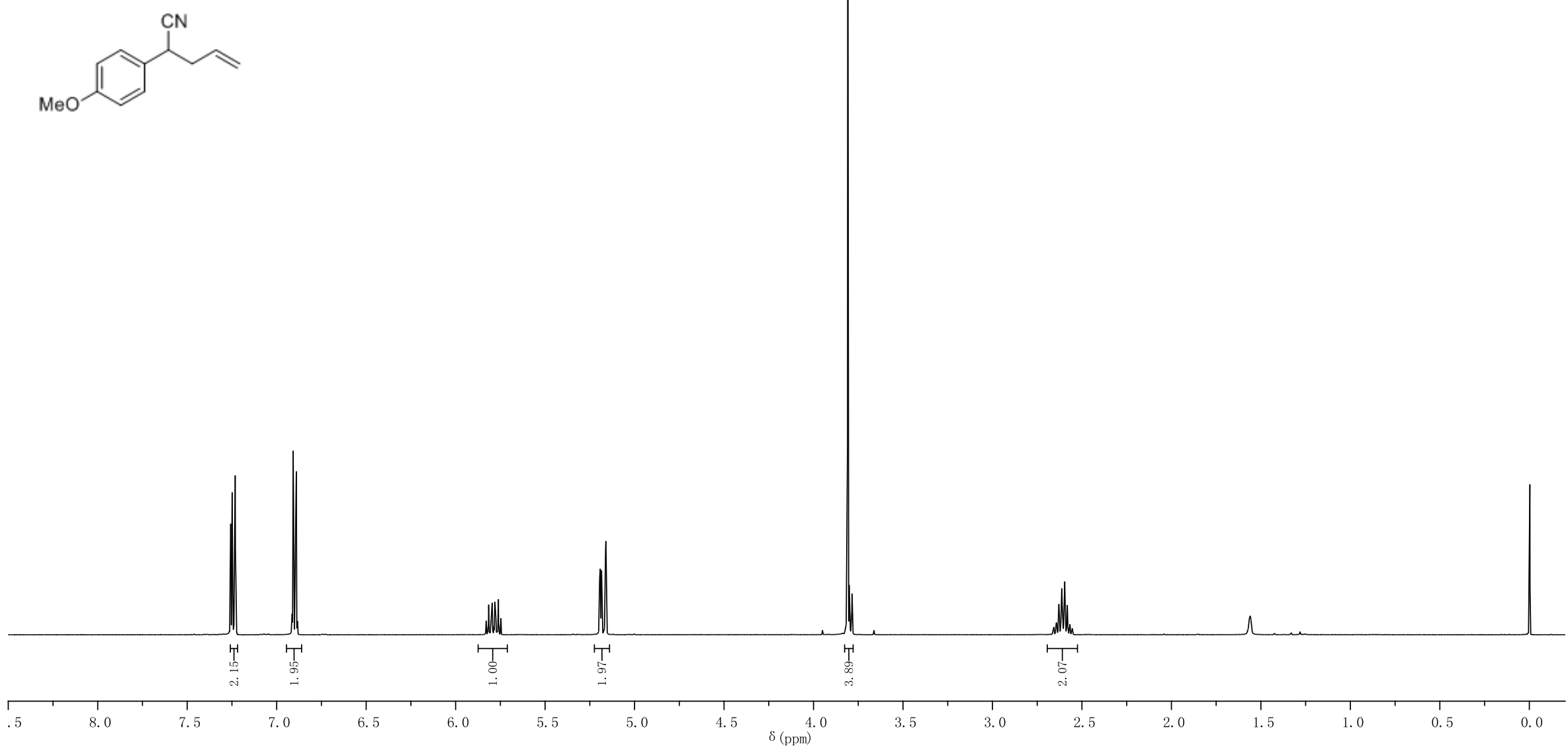
Compound 1j

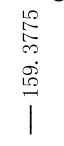

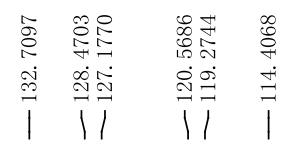

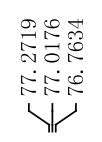

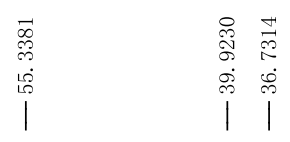

${ }^{13} \mathrm{C}\left\{{ }^{1} \mathrm{H}\right\}$ NMR

$125 \mathrm{MHz}, \mathrm{CDCl}_{3}$

(N)

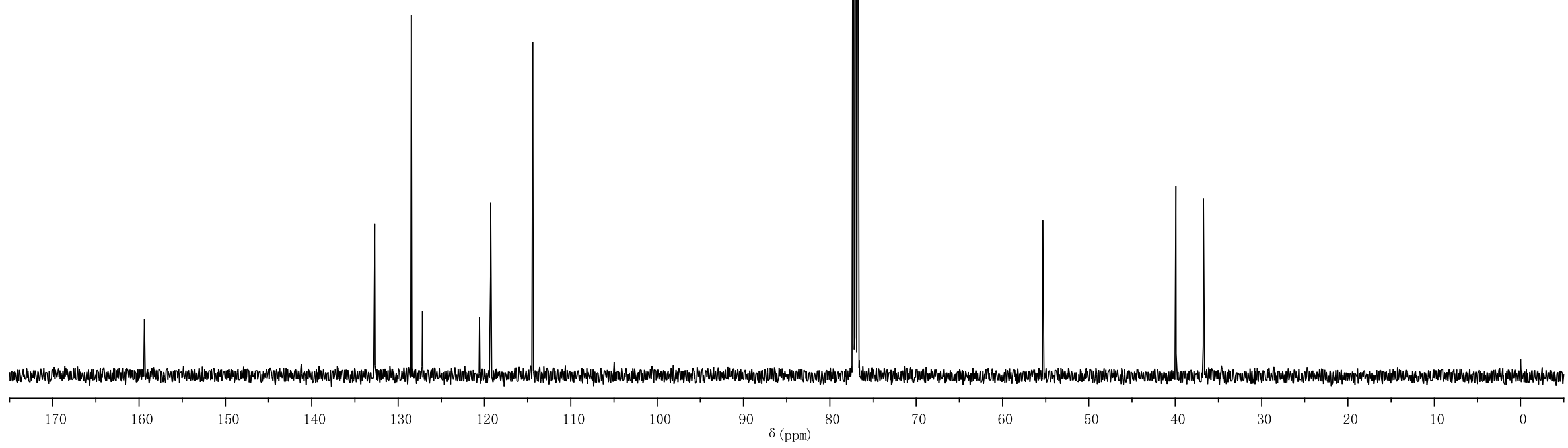

S34 


\section{Compound 1k}

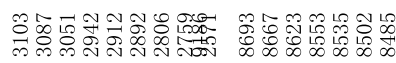

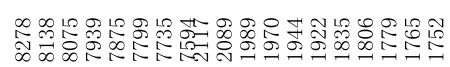

年

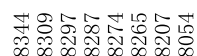

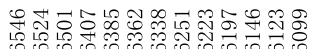

rining ivivie

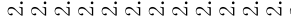

W

$500 \mathrm{MHz}, \mathrm{CDCl}_{3}$
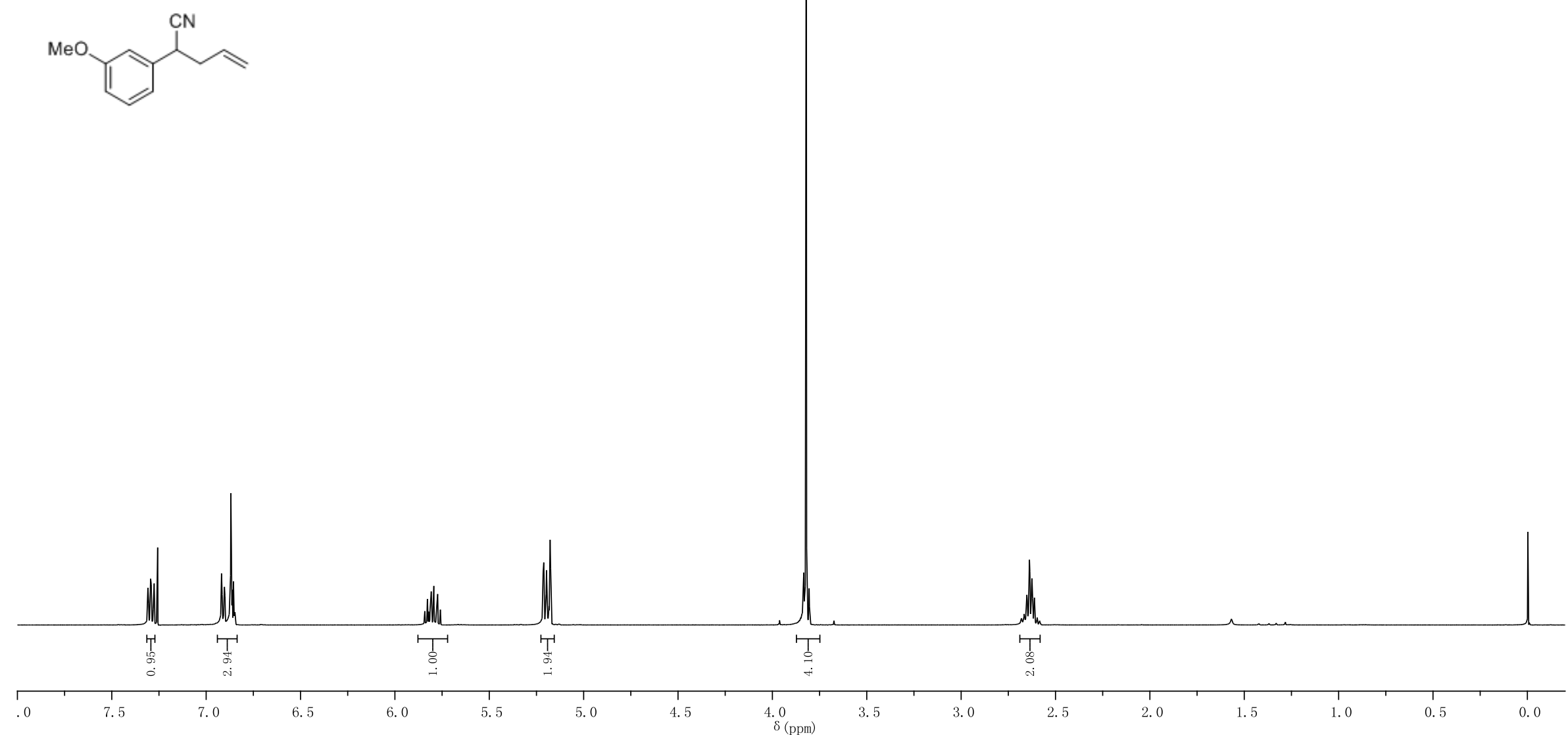
Compound 1k

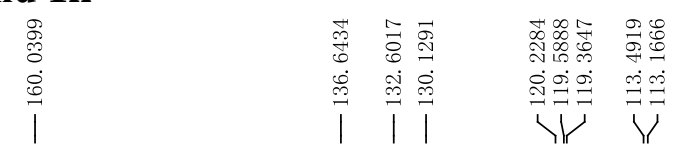

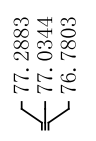

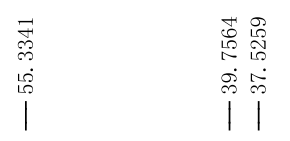

${ }^{13} \mathrm{C}\left\{{ }^{1} \mathrm{H}\right\}$ NMR

$125 \mathrm{MHz}, \mathrm{CDCl}_{3}$
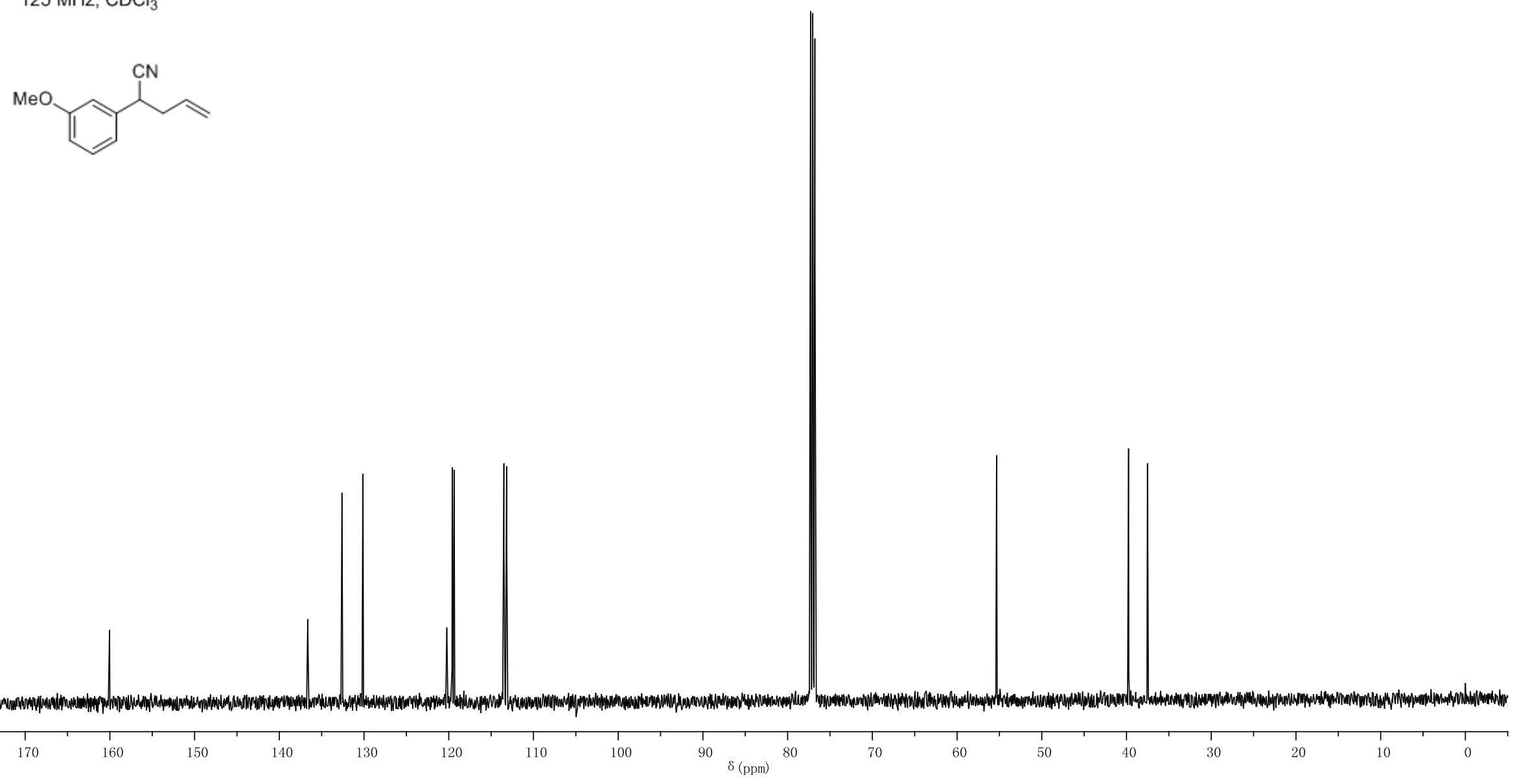

S36 


\section{Compound 11}

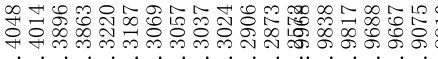

${ }^{1} \mathrm{H}$ NMR

$500 \mathrm{MHz} \mathrm{CDCl}_{3}$
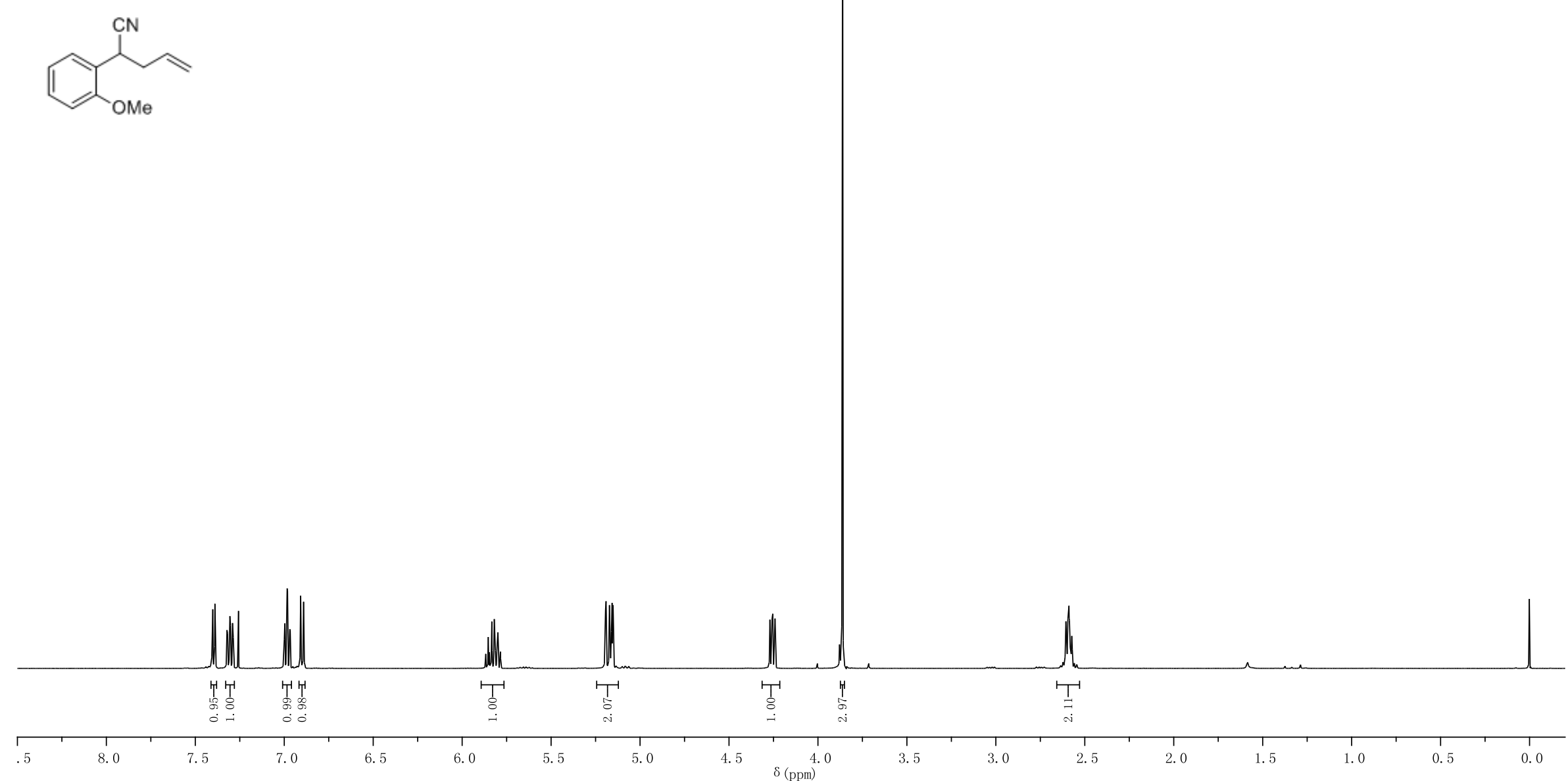


\section{Compound 11}

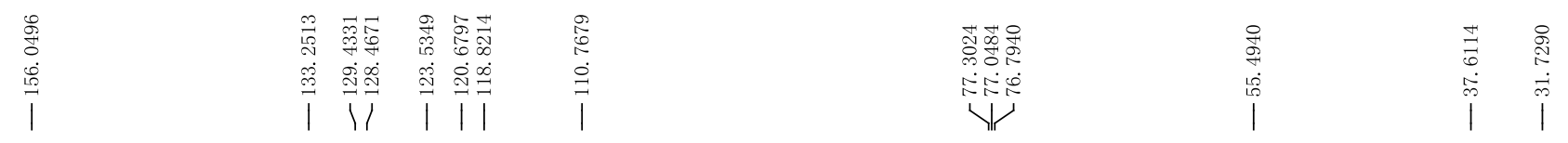

${ }^{13} \mathrm{C}\left\{{ }^{1} \mathrm{H}\right\}$ NMR

$125 \mathrm{MHz}, \mathrm{CDCl}_{3}$
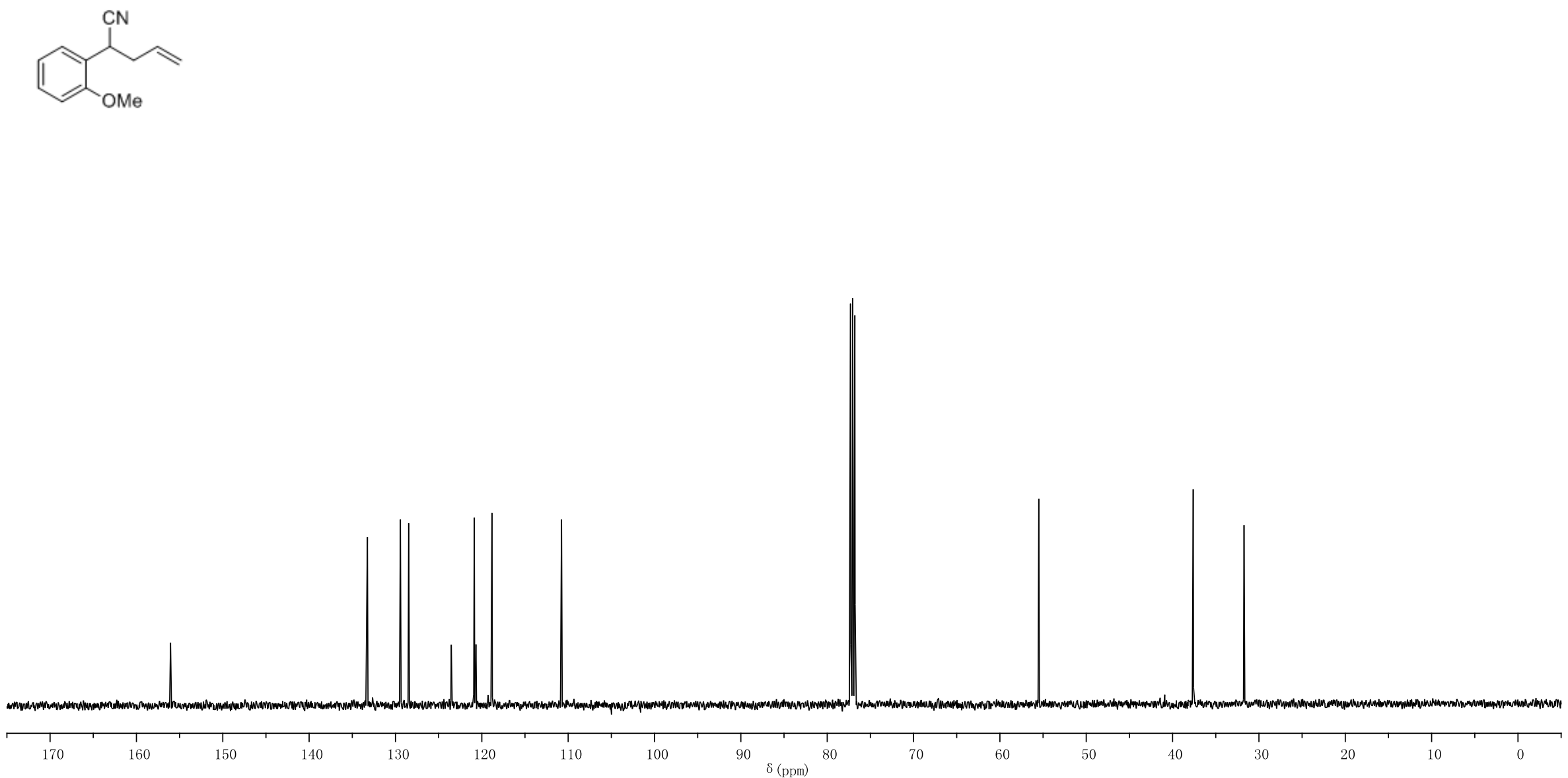


\section{Compound 1m}

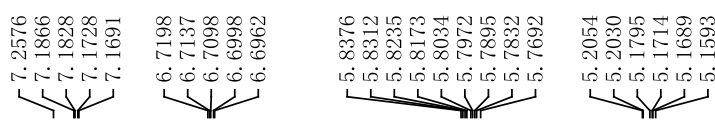

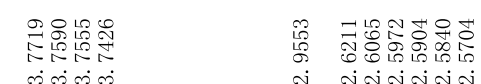

inm

${ }^{1} \mathrm{H}$ NMR

$500 \mathrm{MHz}, \mathrm{CDCl}_{3}$
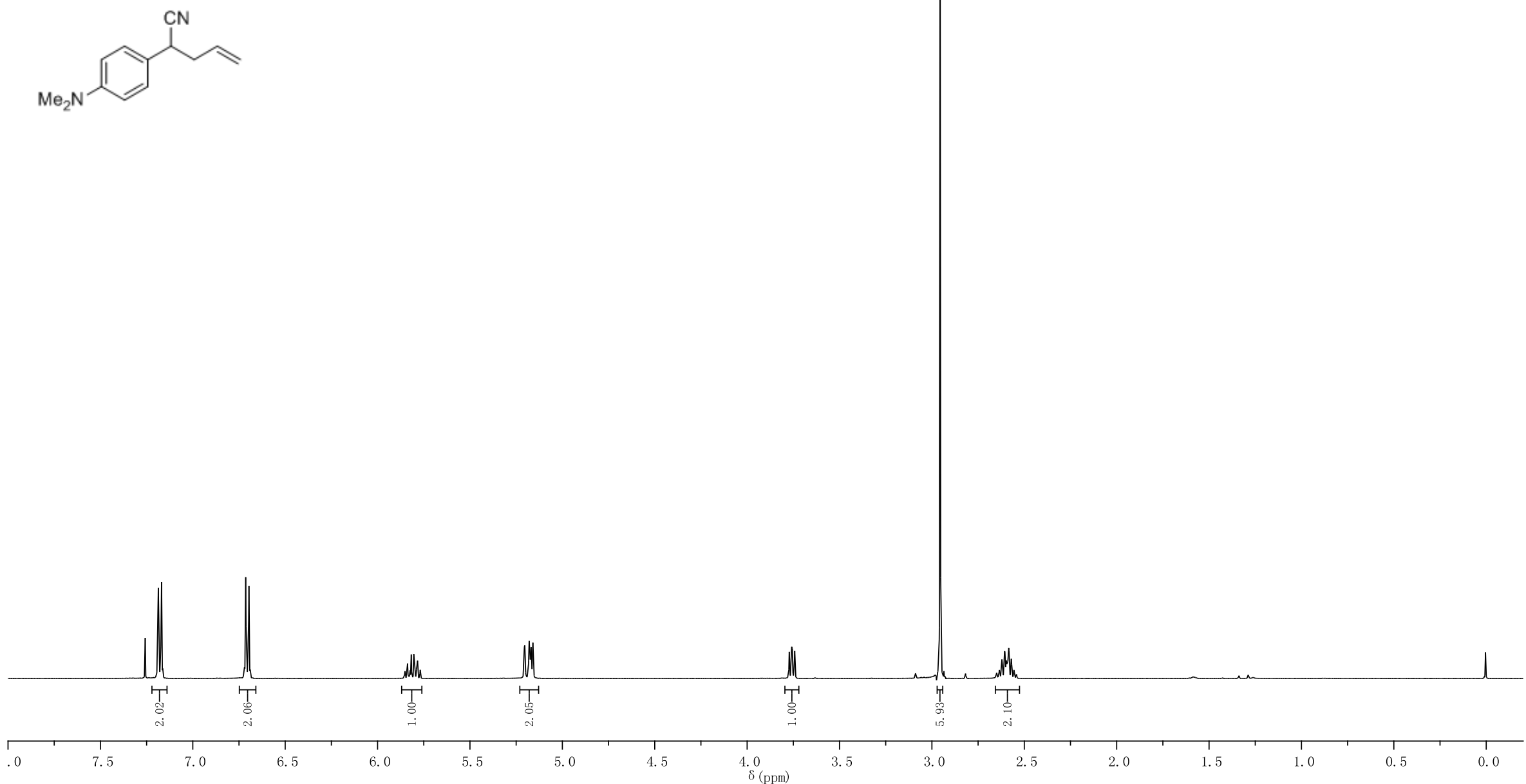


\section{Compound 1m}

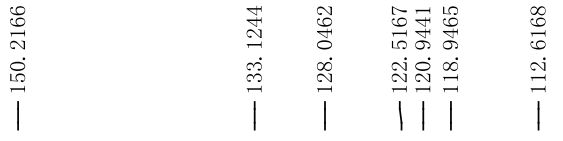

${ }^{13} \mathrm{C}\left\{{ }^{1} \mathrm{H}\right\}$ NMR

$125 \mathrm{MHz}, \mathrm{CDCl}_{3}$

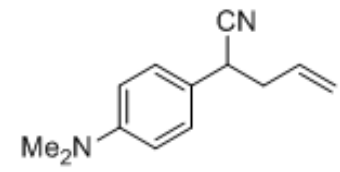

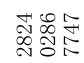

ปิำ

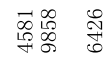

항

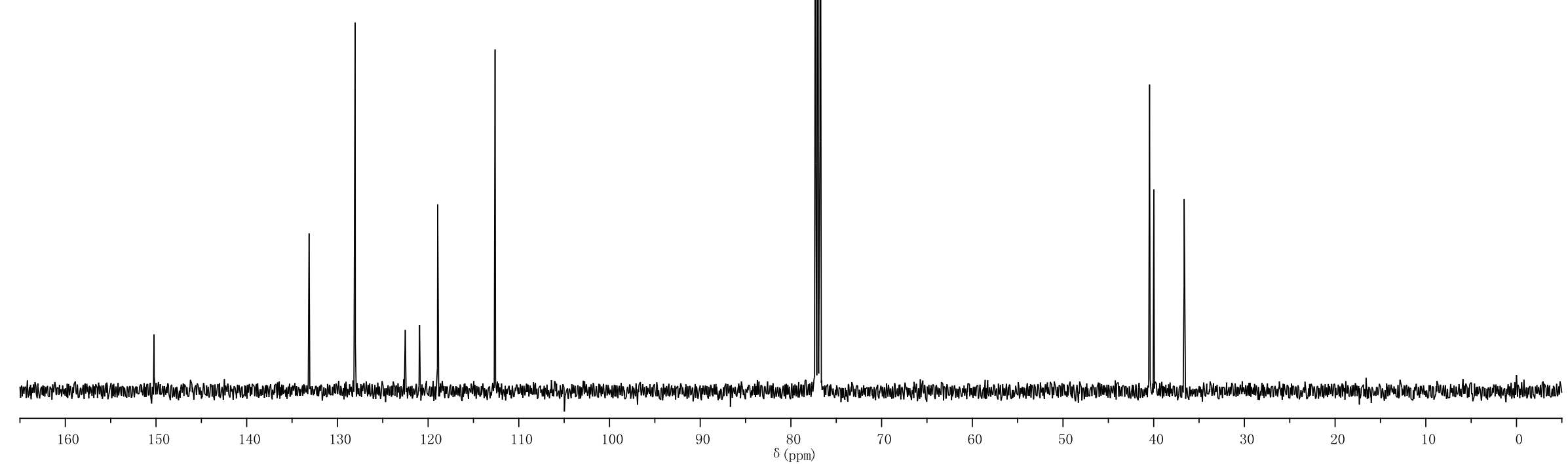




\section{Compound 1n}

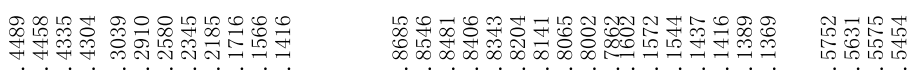

10.

${ }^{1} \mathrm{H}$ NMR

$500 \mathrm{MHz}, \mathrm{CDCl}_{3}$
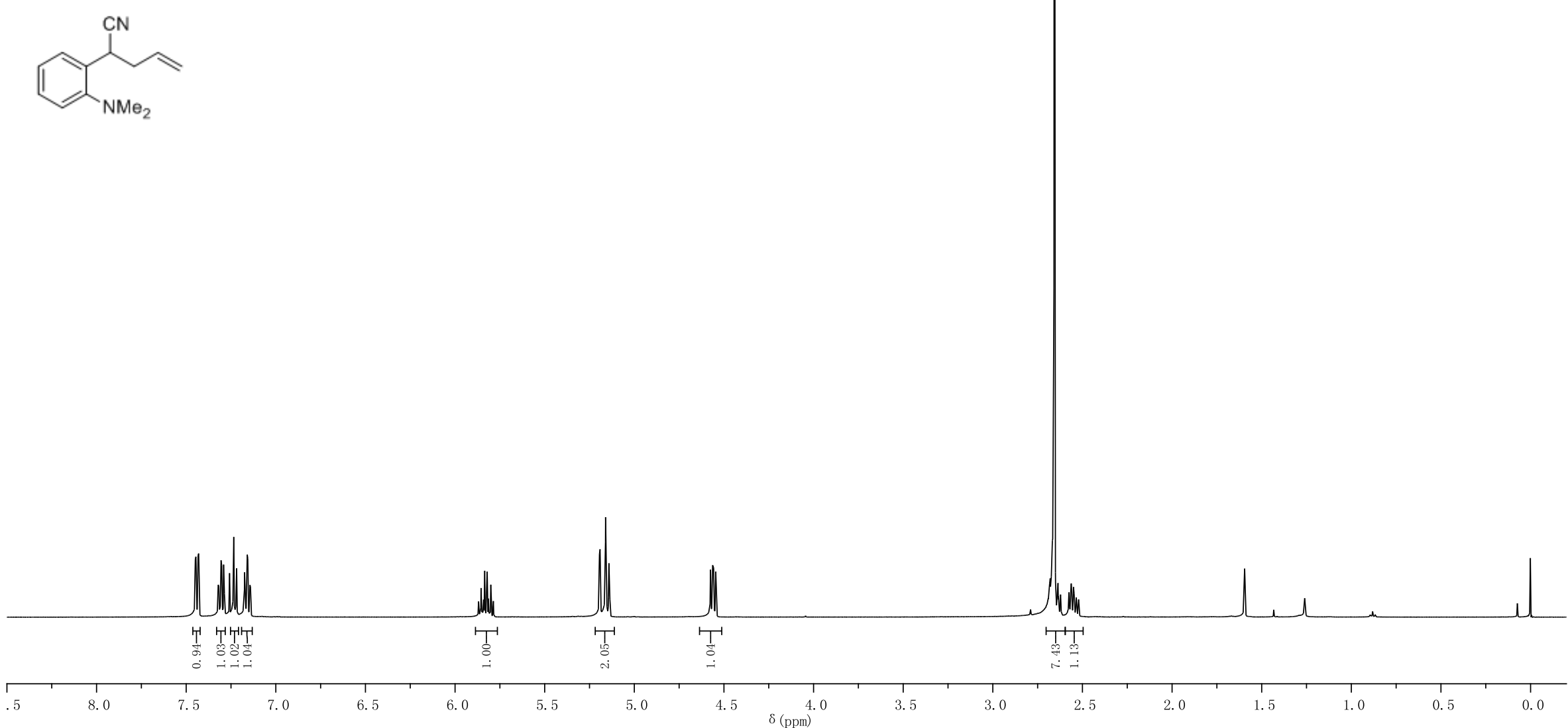


\section{Compound 1n}

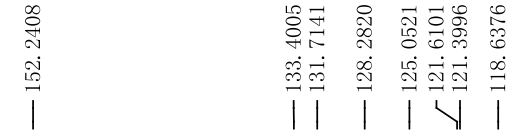

${ }^{13} \mathrm{C}\left\{{ }^{1} \mathrm{H}\right\} \mathrm{NMR}$

$125 \mathrm{MHz}, \mathrm{CDCl}_{3}$
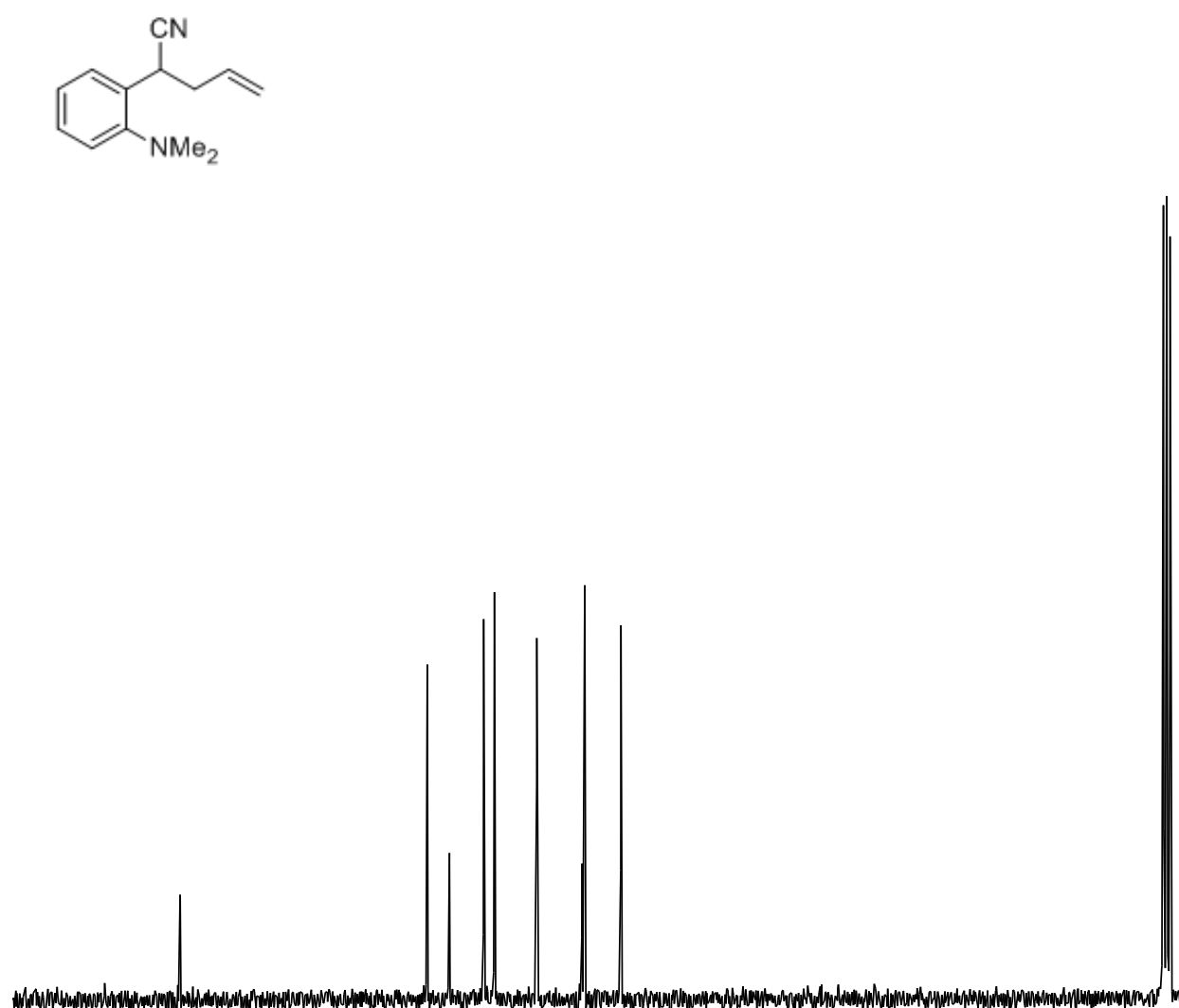

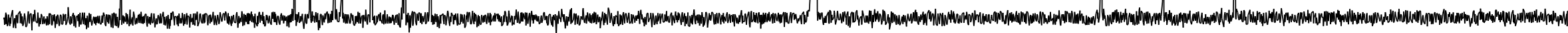

160

150

140

130

120

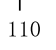

100

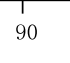

80
$\delta(\mathrm{ppm})$

70

60

50

40

30

20 


\section{Compound 1o}

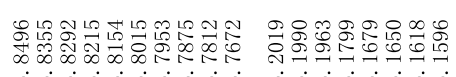

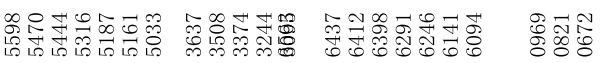

نun

${ }^{1} \mathrm{H}$ NMR

$500 \mathrm{MHz}, \mathrm{CDCl}_{3}$
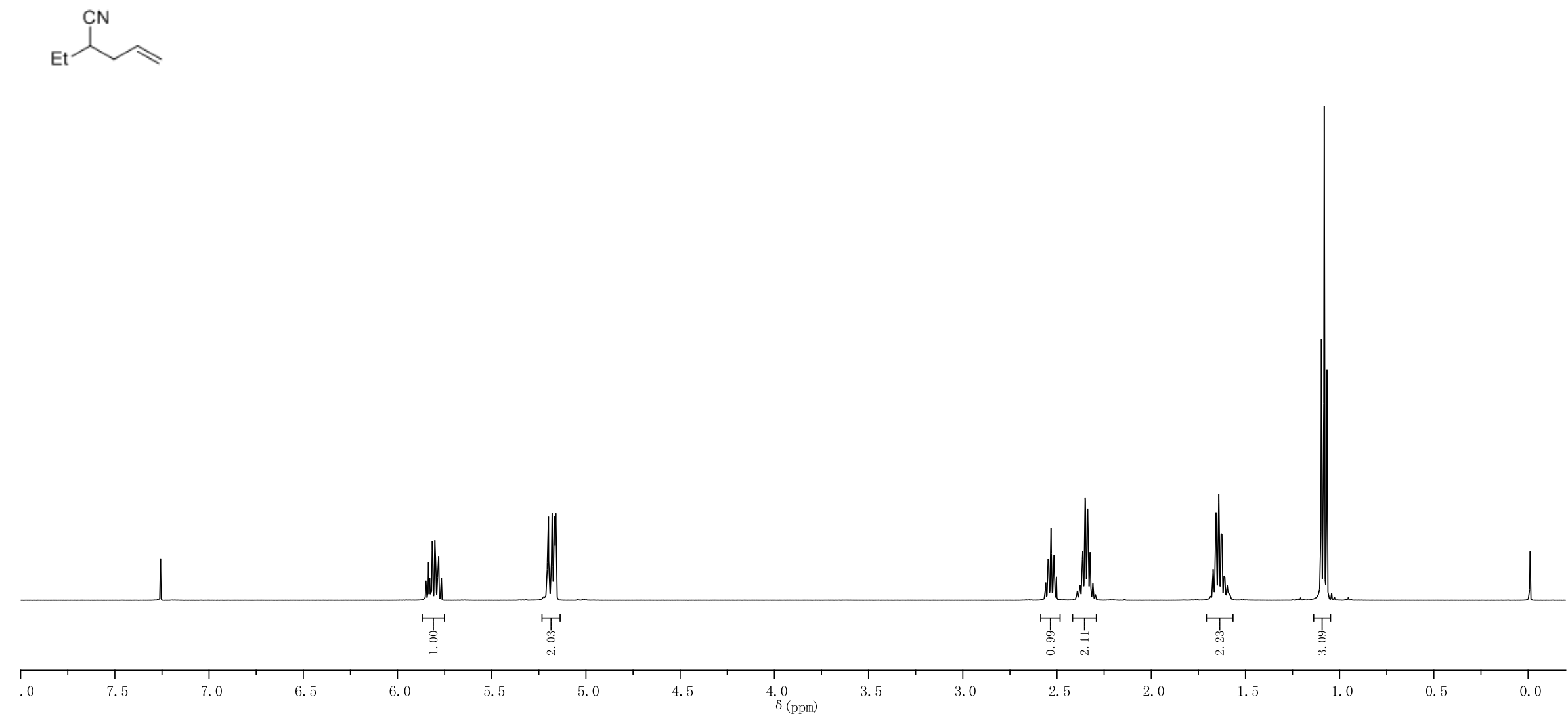
Compound 10

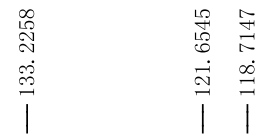

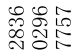

ปิ

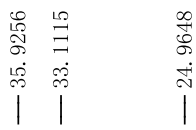

${ }^{13} \mathrm{C}\left\{{ }^{1} \mathrm{H}\right\}$ NMR

$125 \mathrm{MHz}, \mathrm{CDCl}_{3}$
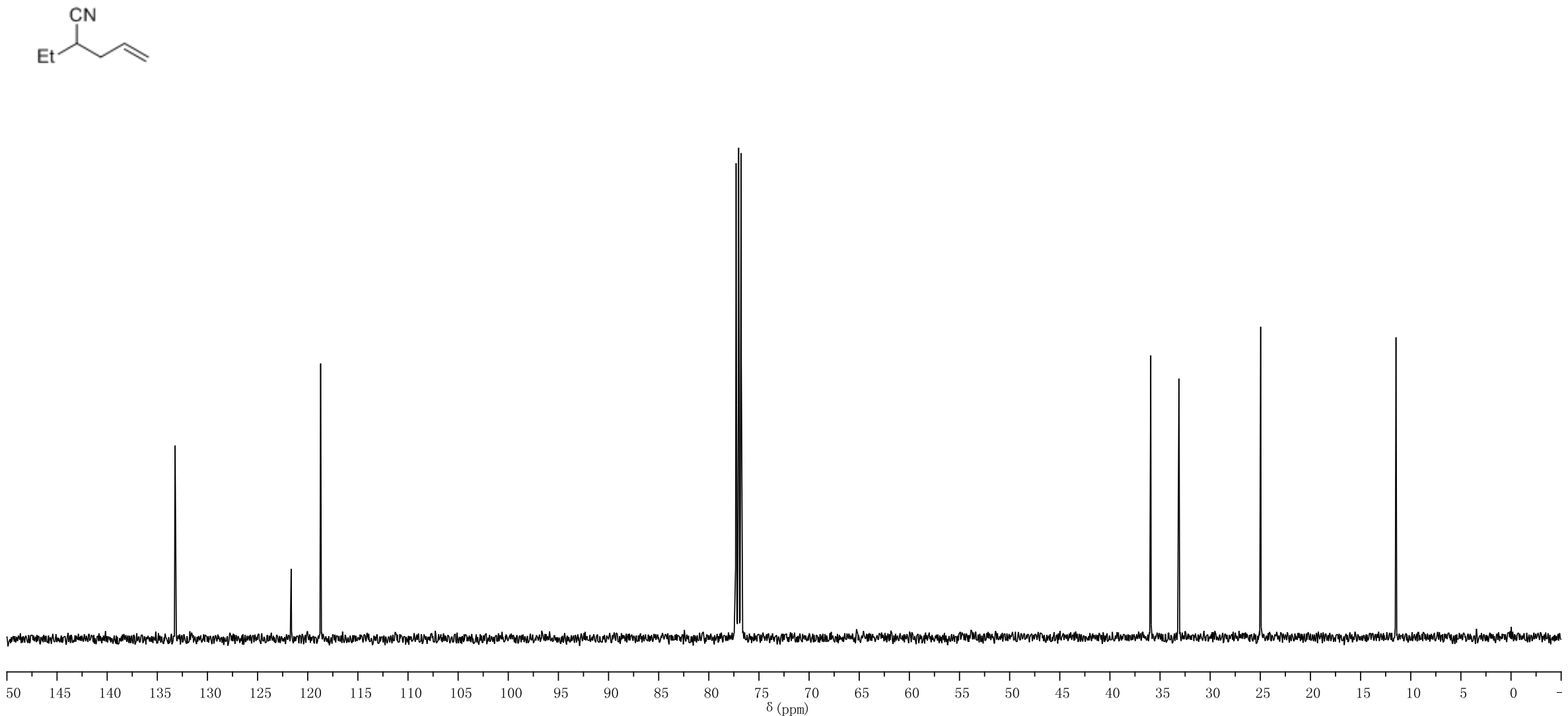


\section{Compound 1p}

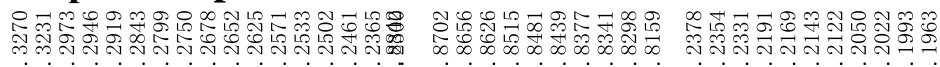

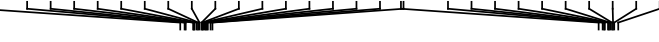

$500 \mathrm{MHz}, \mathrm{CDCl}_{3}$
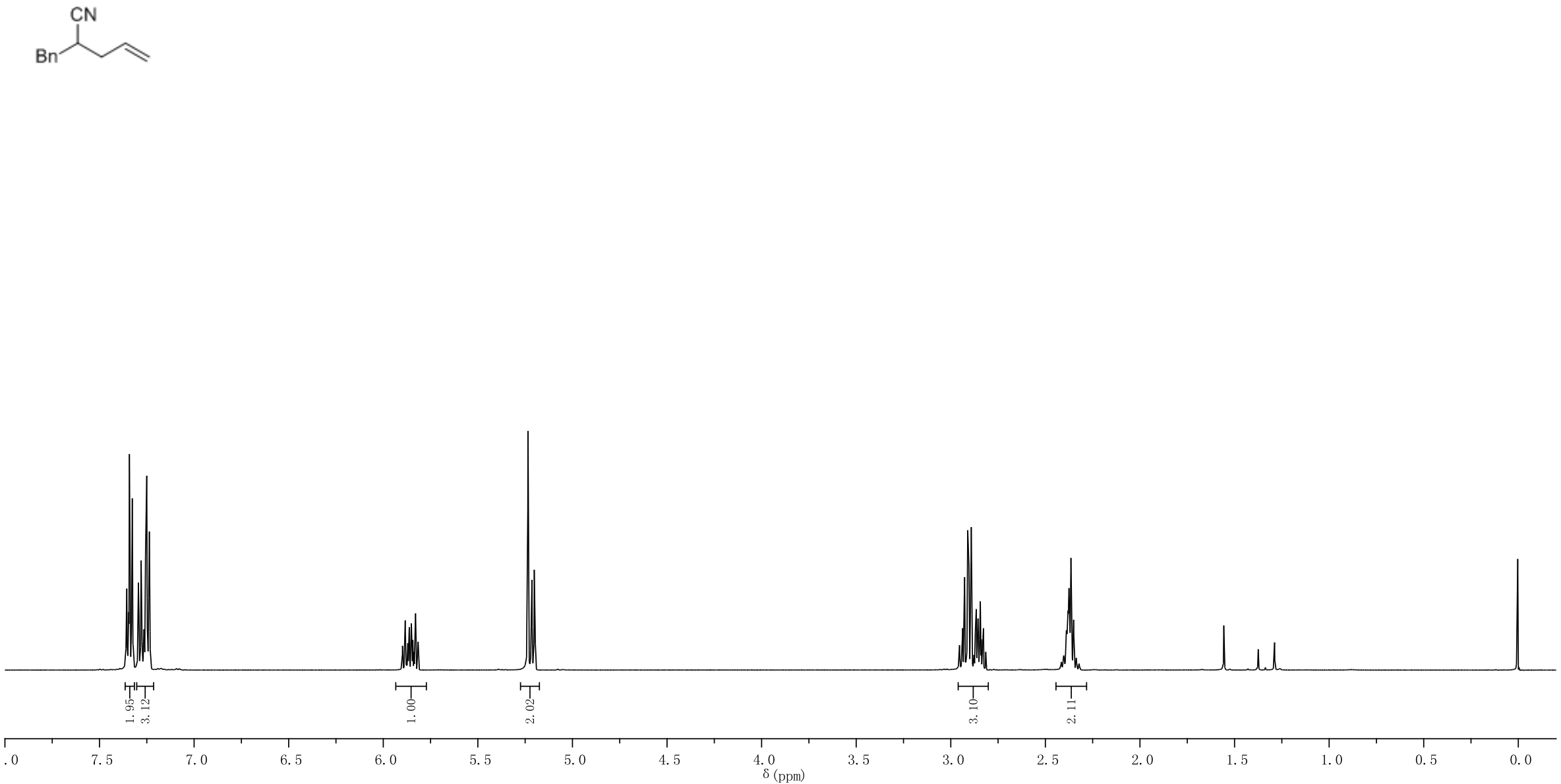


\section{Compound 1p

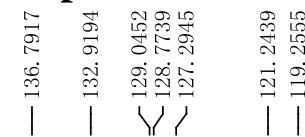

的帛嵒

| | |

${ }^{13} \mathrm{C}\left\{{ }^{1} \mathrm{H}\right\}$ NMR

$125 \mathrm{MHz}, \mathrm{CDCl}_{3}$
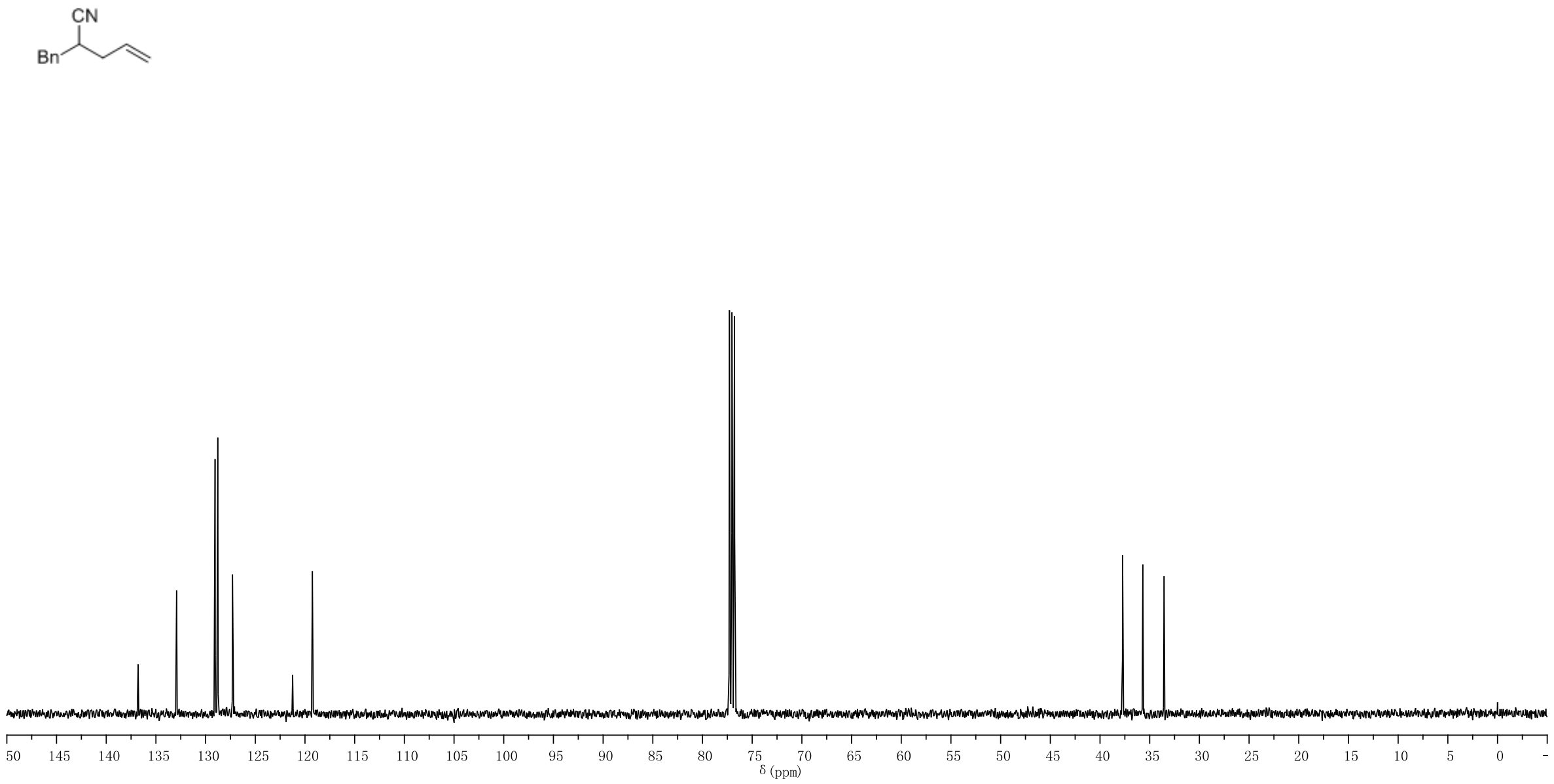


\section{Compound 1q}

i

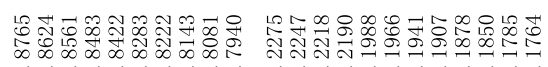
10.

$500 \mathrm{MHz}, \mathrm{CDCl}_{3}$
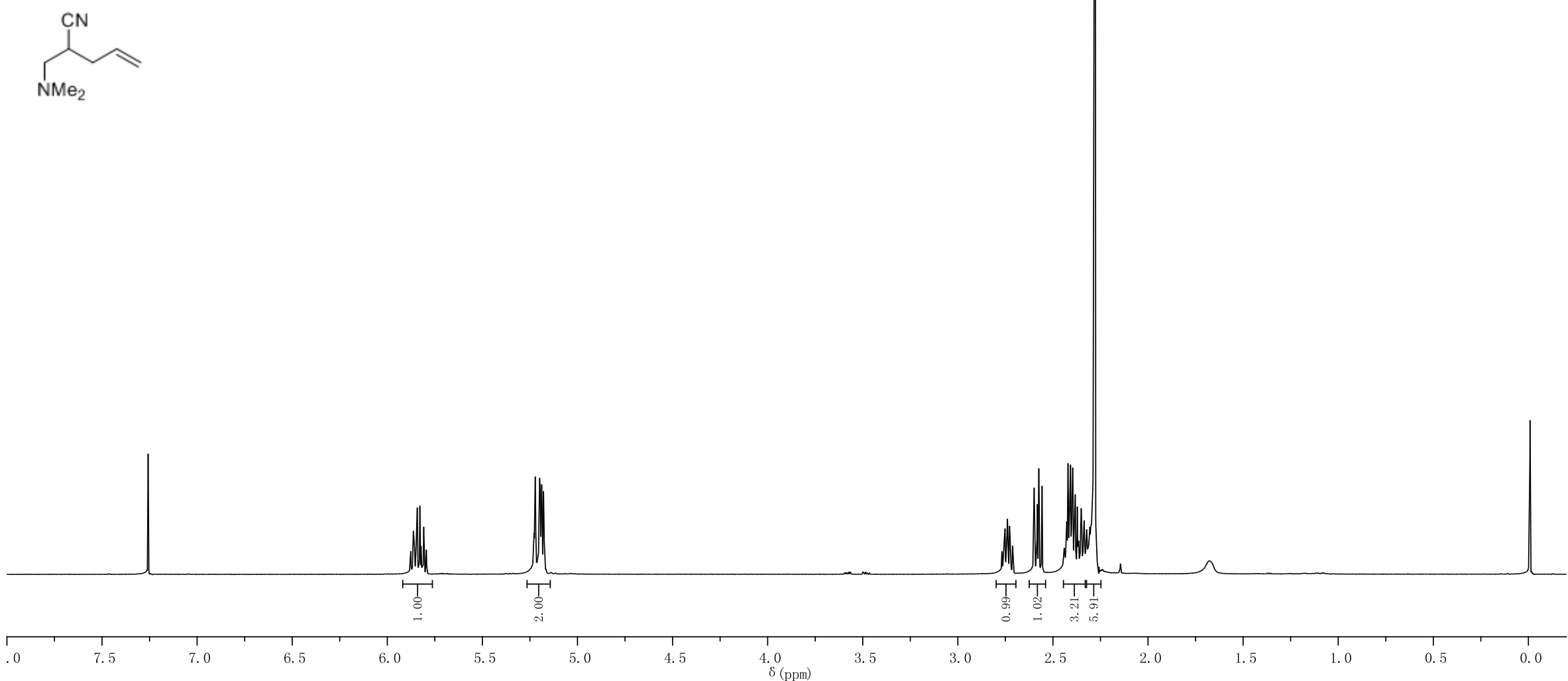
Compound 1q

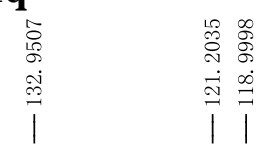

${ }^{13} \mathrm{C}\left\{{ }^{1} \mathrm{H}\right\}$ NMR

$125 \mathrm{MHz}, \mathrm{CDCl}_{3}$
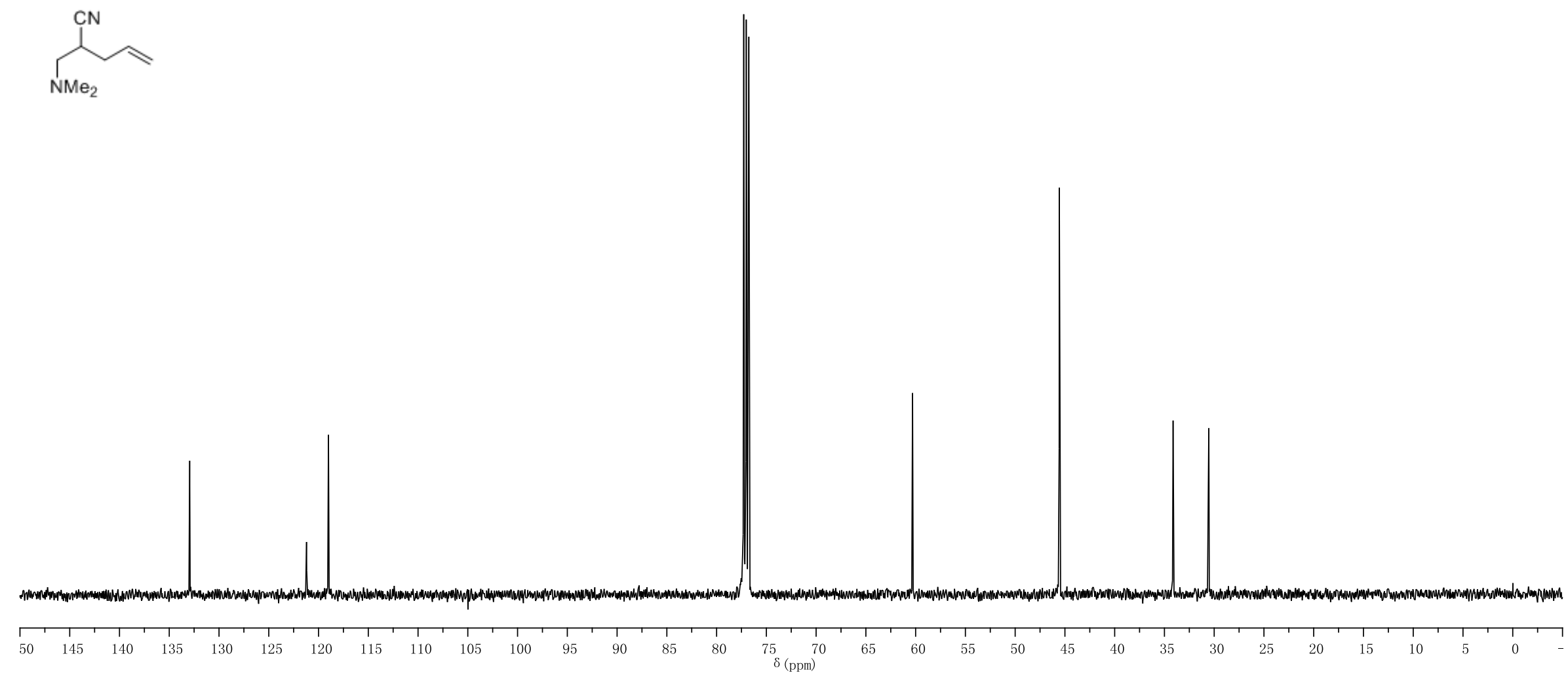

$\begin{array}{lllllllllllllllllllllll}50 & 145 & 140 & 135 & 130 & 125 & 120 & 115 & 110 & 1 & 105 & 100 & 95 & 90 & 85 & 80 & 75 & 70 \\ \delta(\mathrm{ppm})\end{array}$ 


\section{Compound 1r}

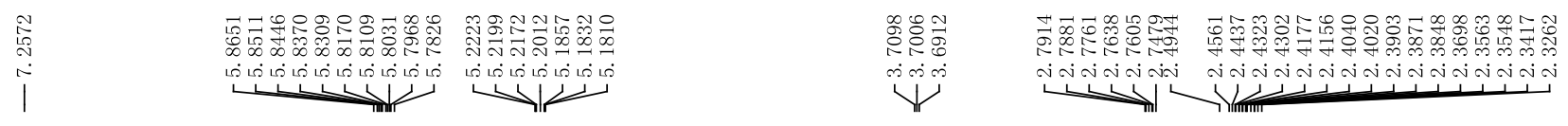

${ }^{1} \mathrm{H}$ NMR

$500 \mathrm{MHz}, \mathrm{CDCl}_{3}$
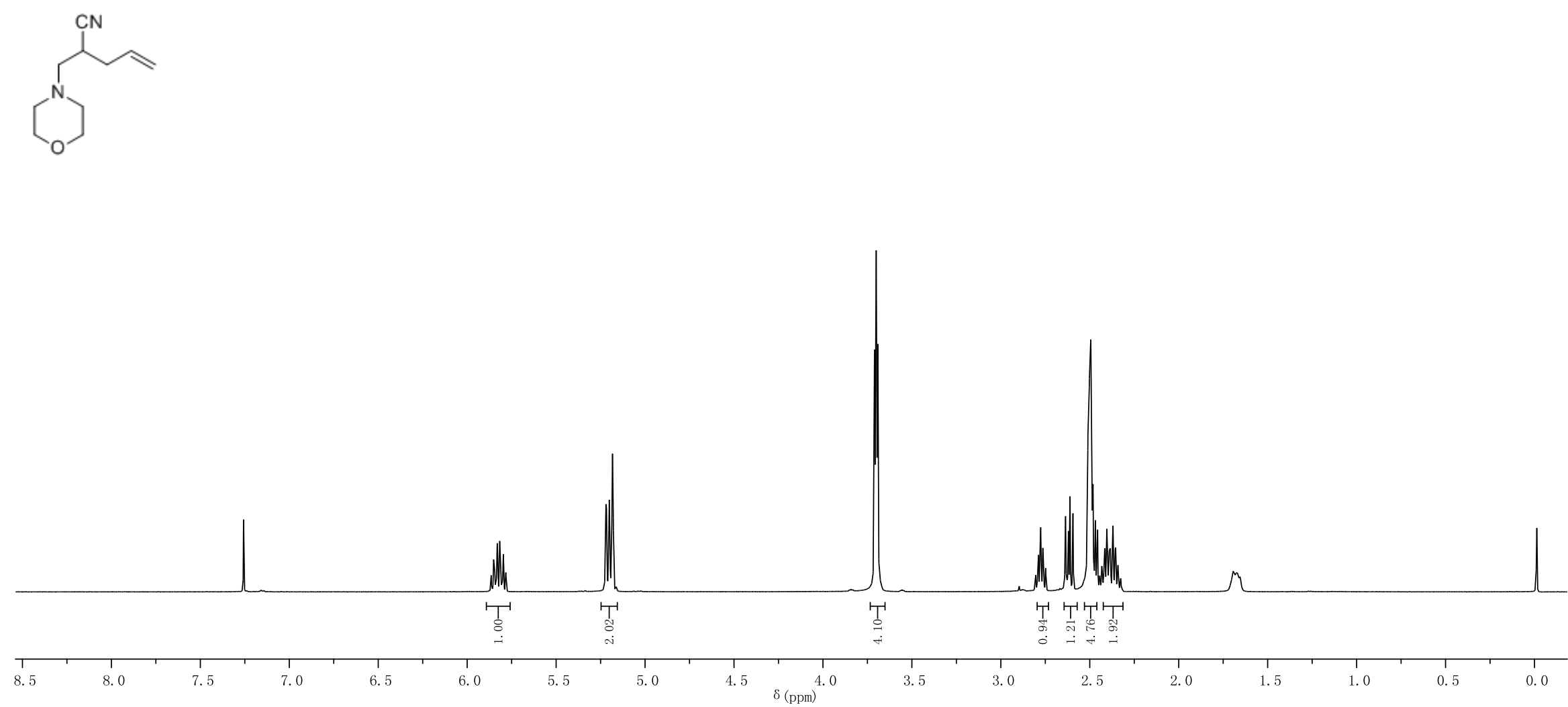


\section{Compound 1r}

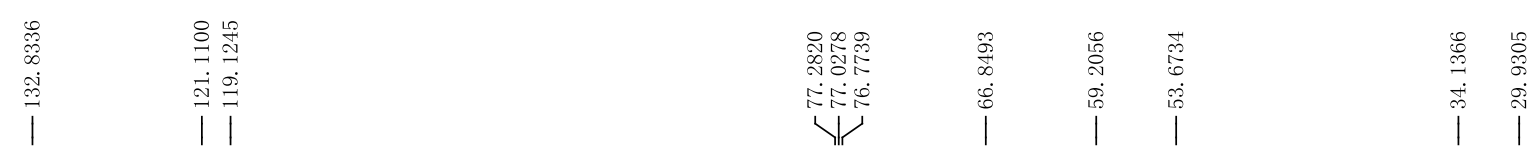

${ }^{13} \mathrm{C}\left\{{ }^{1} \mathrm{H}\right\}$ NMR

$125 \mathrm{MHz}, \mathrm{CDCl}_{3}$
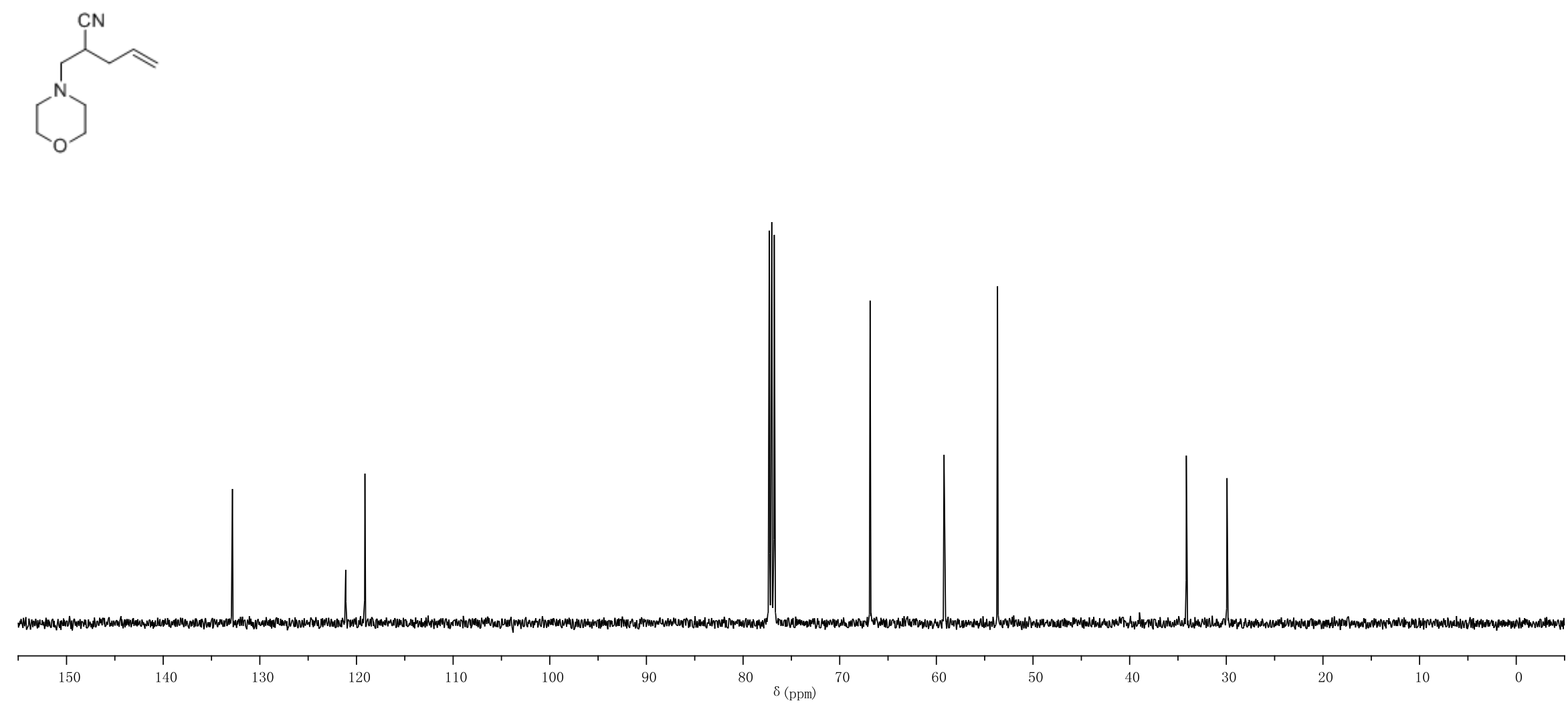


\section{Compound 1s}

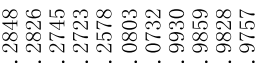

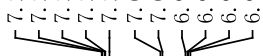

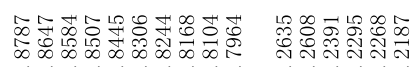

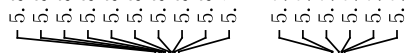

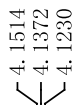

${ }^{1} \mathrm{H}$ NMR

$500 \mathrm{MHz}, \mathrm{CDCl}_{3}$
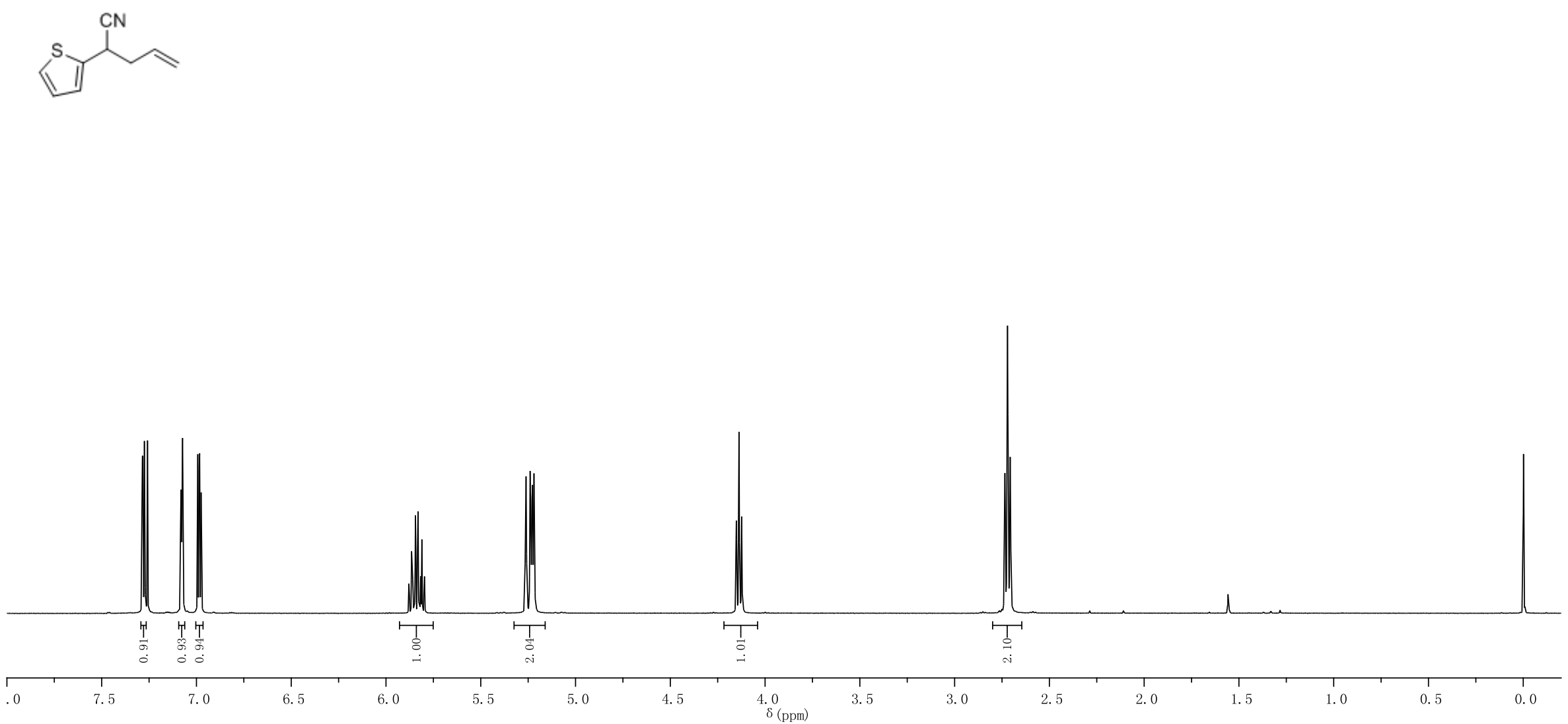
Compound 1s

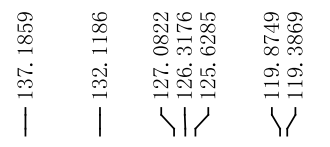

${ }^{13} \mathrm{C}\left\{{ }^{1} \mathrm{H}\right\}$ NMR

$125 \mathrm{MHz}, \mathrm{CDCl}_{3}$
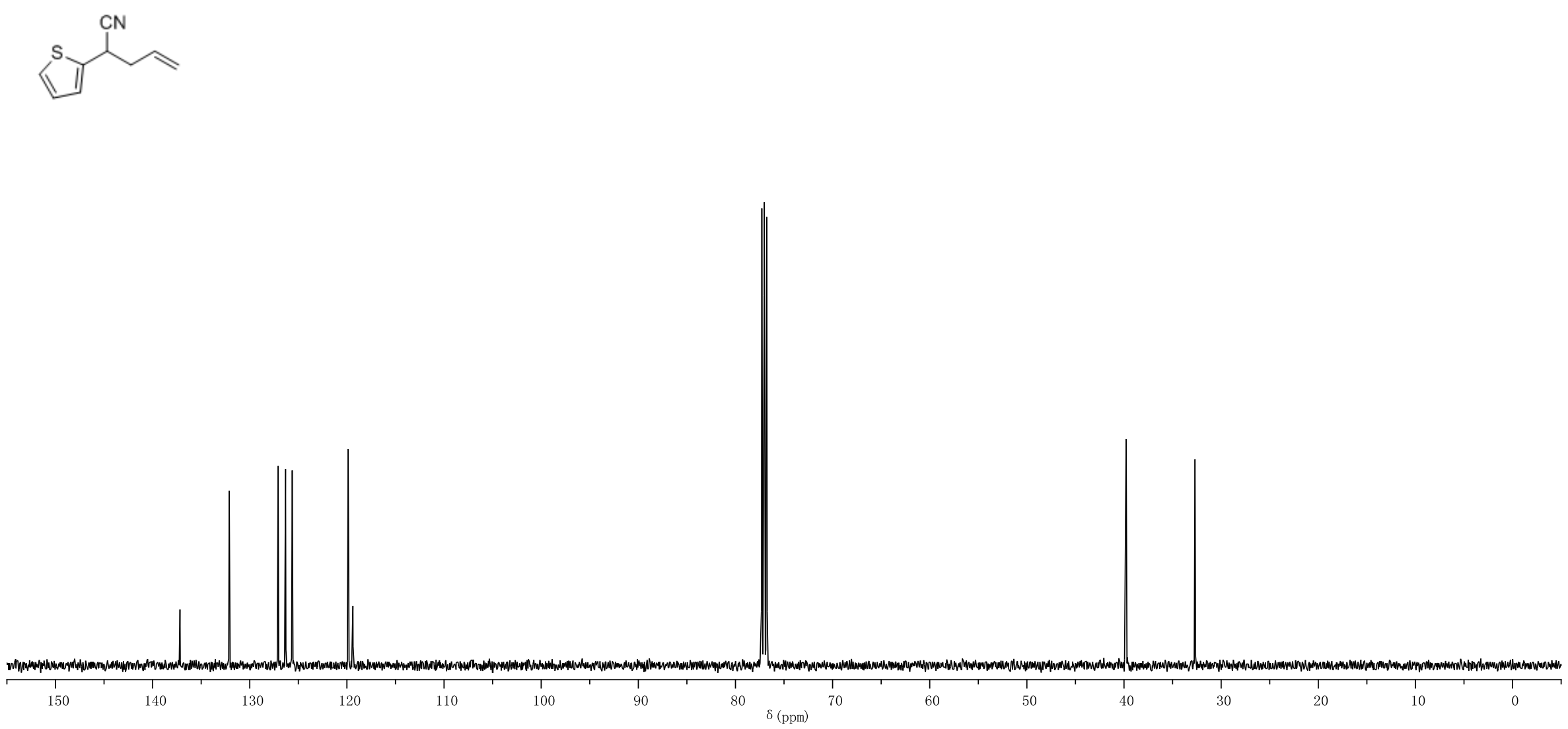


\section{Compound 1t}
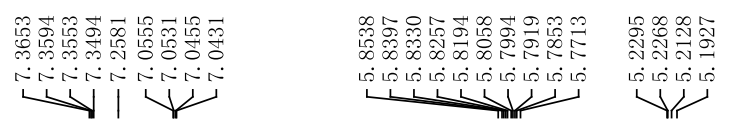

\section{${ }^{1} \mathrm{H}$ NMR}

$500 \mathrm{MHz}, \mathrm{CDCl}_{3}$
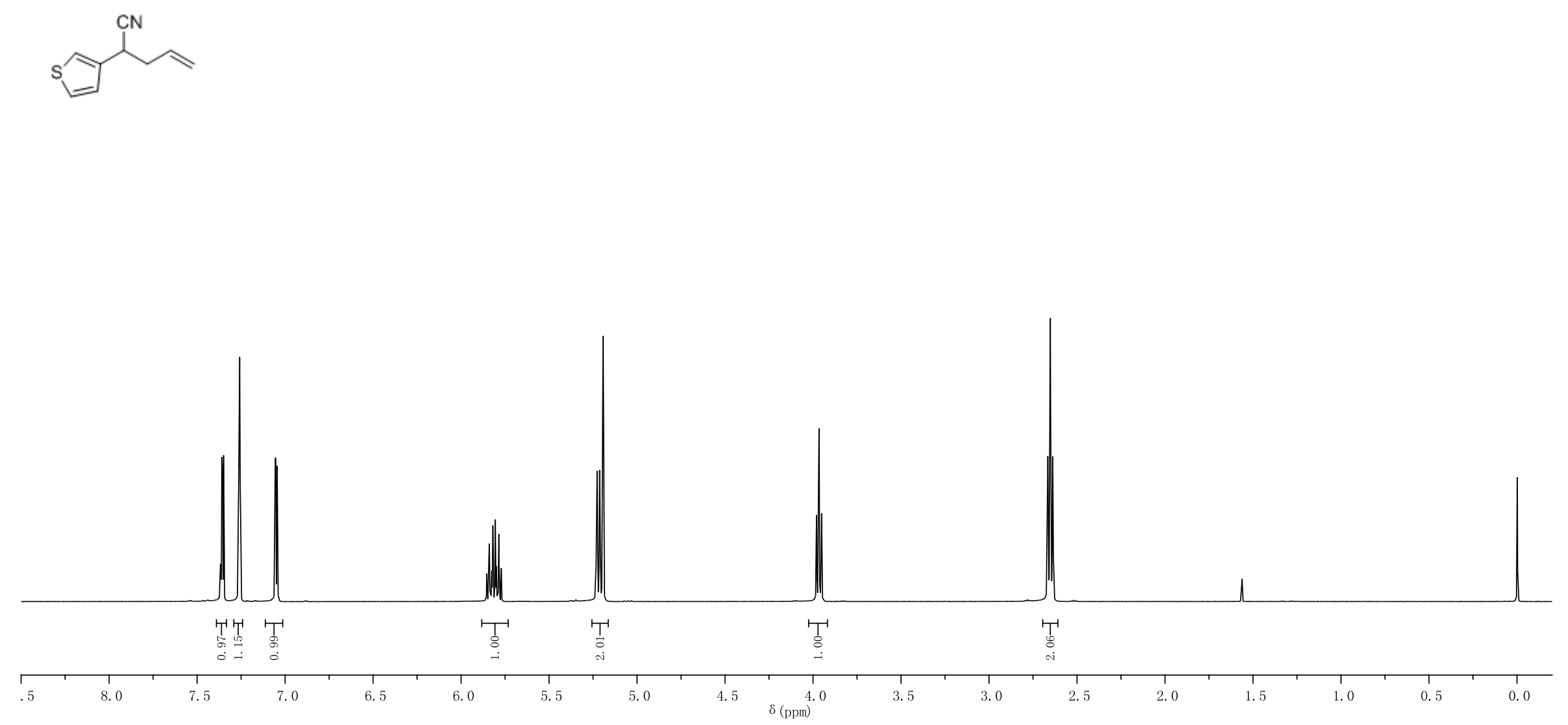
Compound It

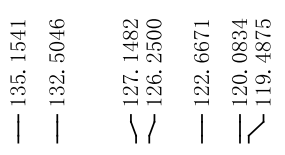

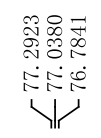

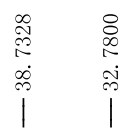

${ }^{13} \mathrm{C}\left\{{ }^{1} \mathrm{H}\right\}$ NMR

$125 \mathrm{MHz} \mathrm{CDCl}_{3}$
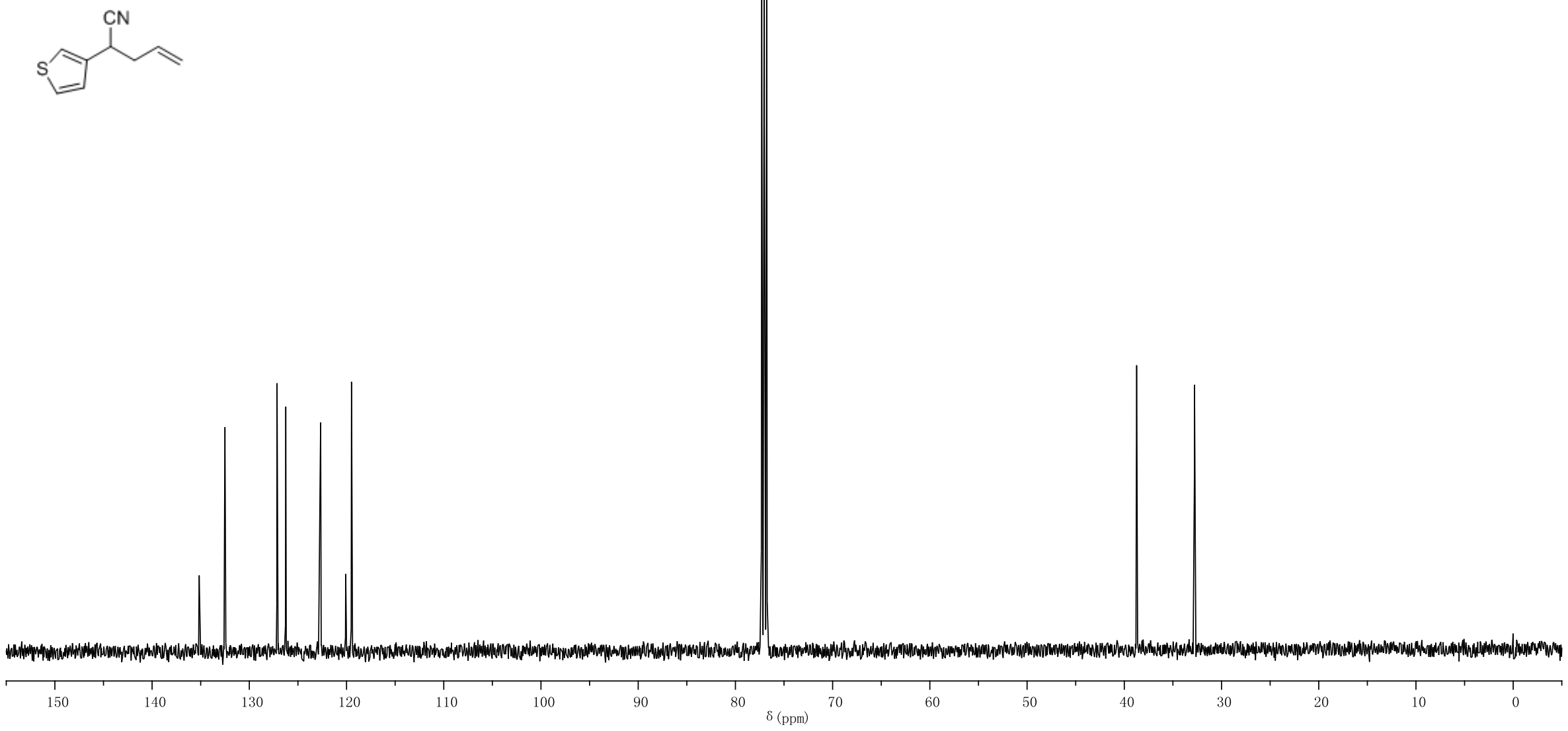

S54 


\section{Compound 1u}

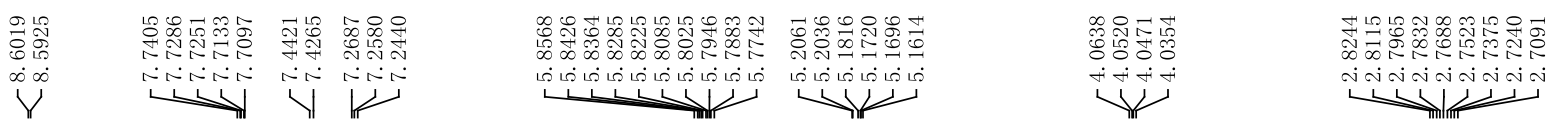

${ }^{1} \mathrm{H}$ NMR

$500 \mathrm{MHz}, \mathrm{CDCl}_{3}$
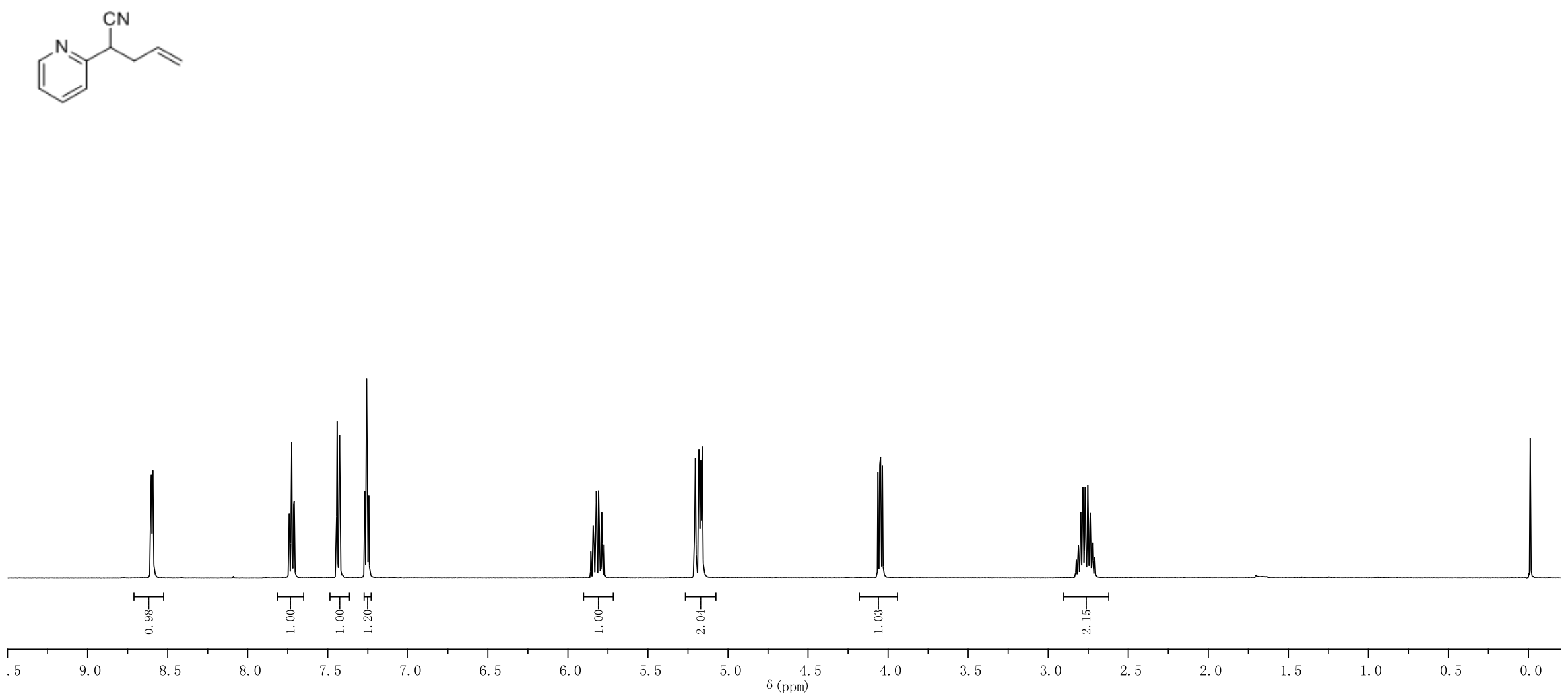
Compound 1u

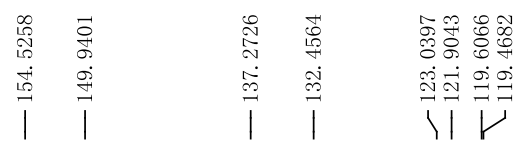

${ }^{13} \mathrm{C}\left\{{ }^{1} \mathrm{H}\right\}$ NMR

$125 \mathrm{MHz}, \mathrm{CDCl}_{3}$
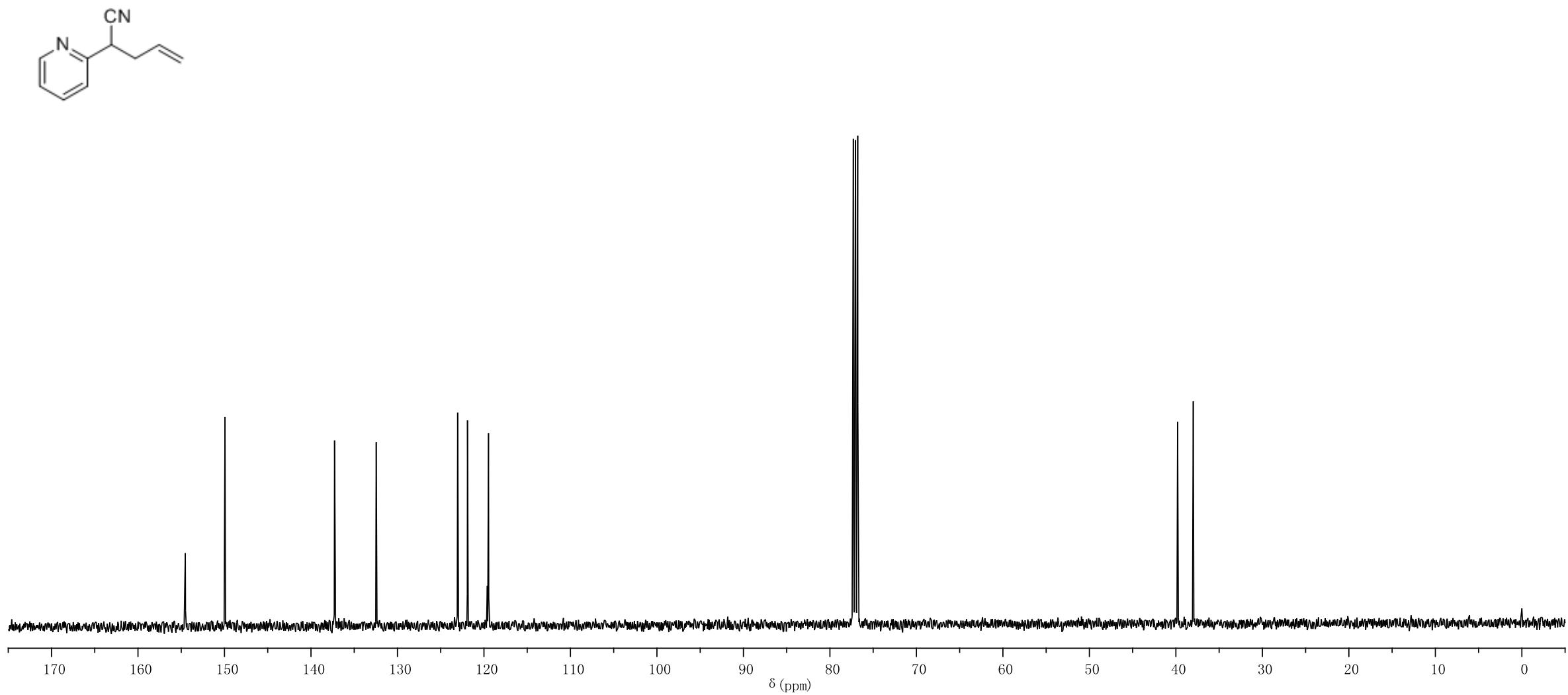


\section{Compound 1v}

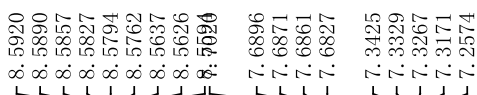

${ }^{1} \mathrm{H}$ NMR

$500 \mathrm{MHz}, \mathrm{CDCl}_{3}$
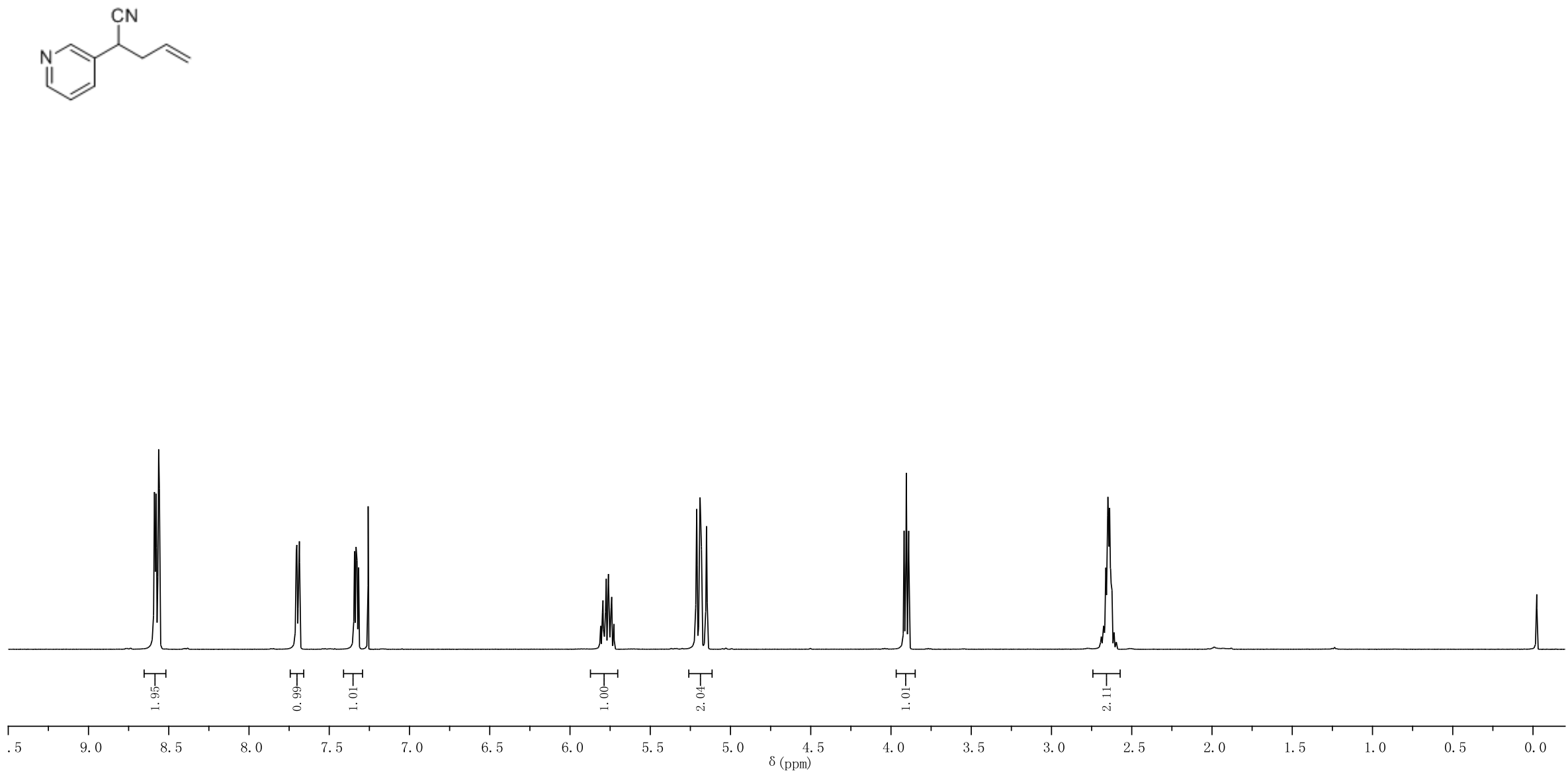


\section{Compound 1v}

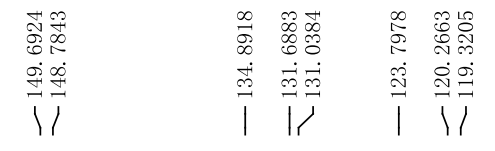

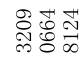

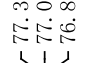

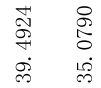

|

${ }^{13} \mathrm{C}\left\{{ }^{1} \mathrm{H}\right\}$ NMR

$125 \mathrm{MHz}, \mathrm{CDCl}_{3}$
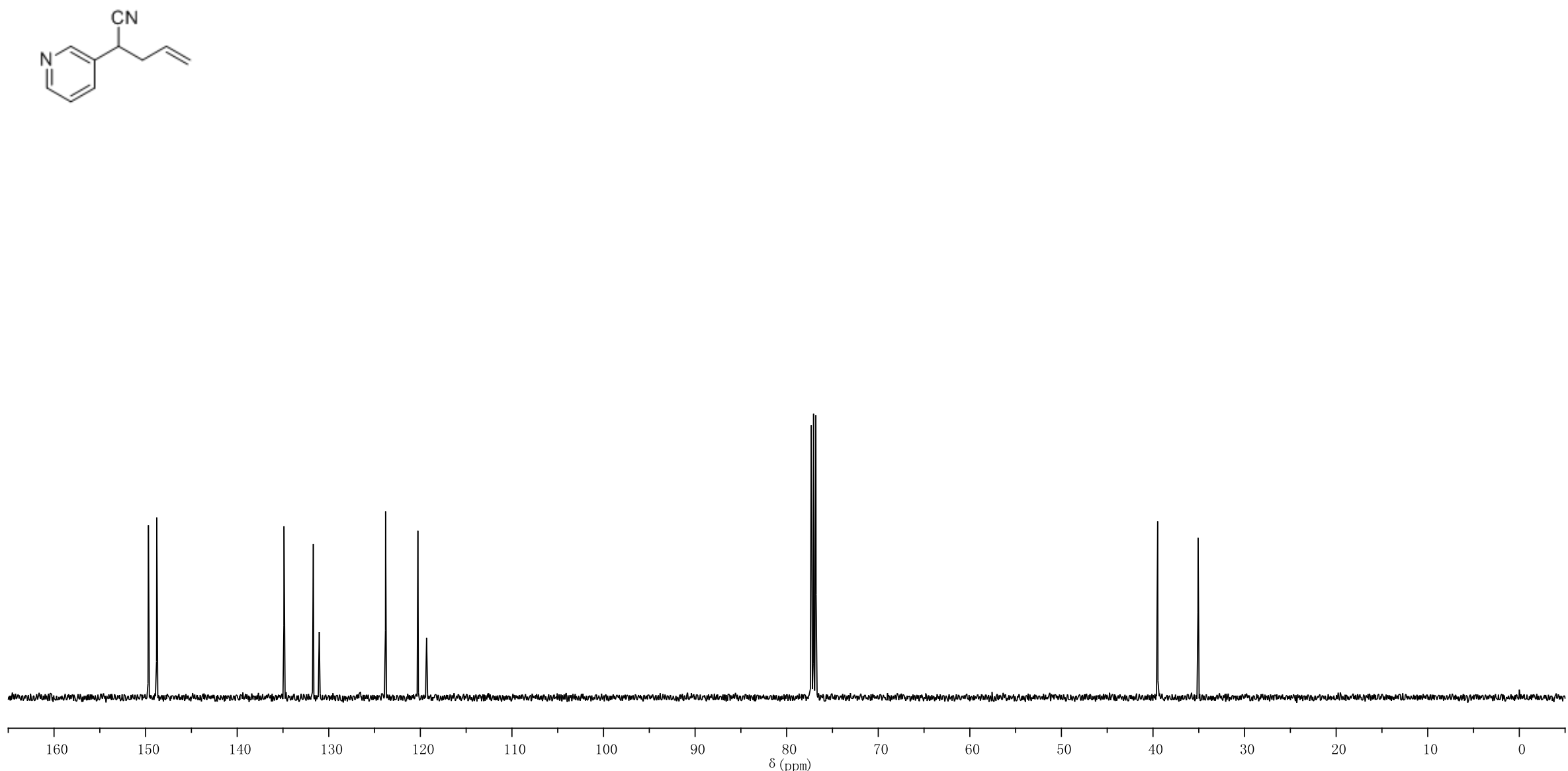


\section{Compound 1w}

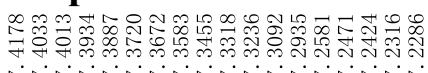

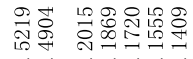

il

${ }^{1} \mathrm{H}$ NMR

$500 \mathrm{MHz}, \mathrm{CDCl}_{3}$
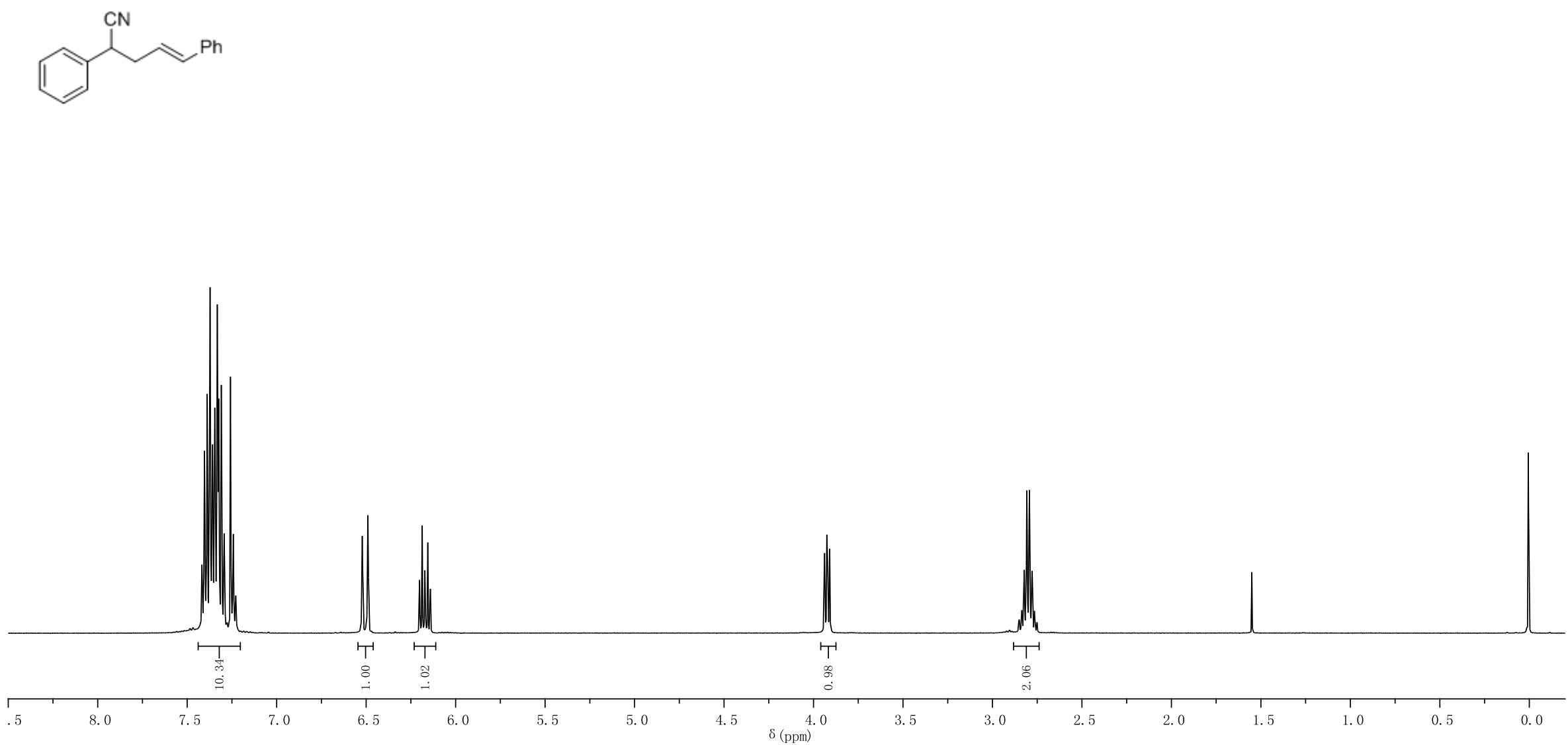


\section{Compound 1w}

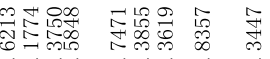

$$
\begin{aligned}
& \text { க. } \\
& \text { ओर }
\end{aligned}
$$

${ }^{13} \mathrm{C}\left\{{ }^{1} \mathrm{H}\right\}$ NMR

$125 \mathrm{MHz}, \mathrm{CDCl}_{3}$
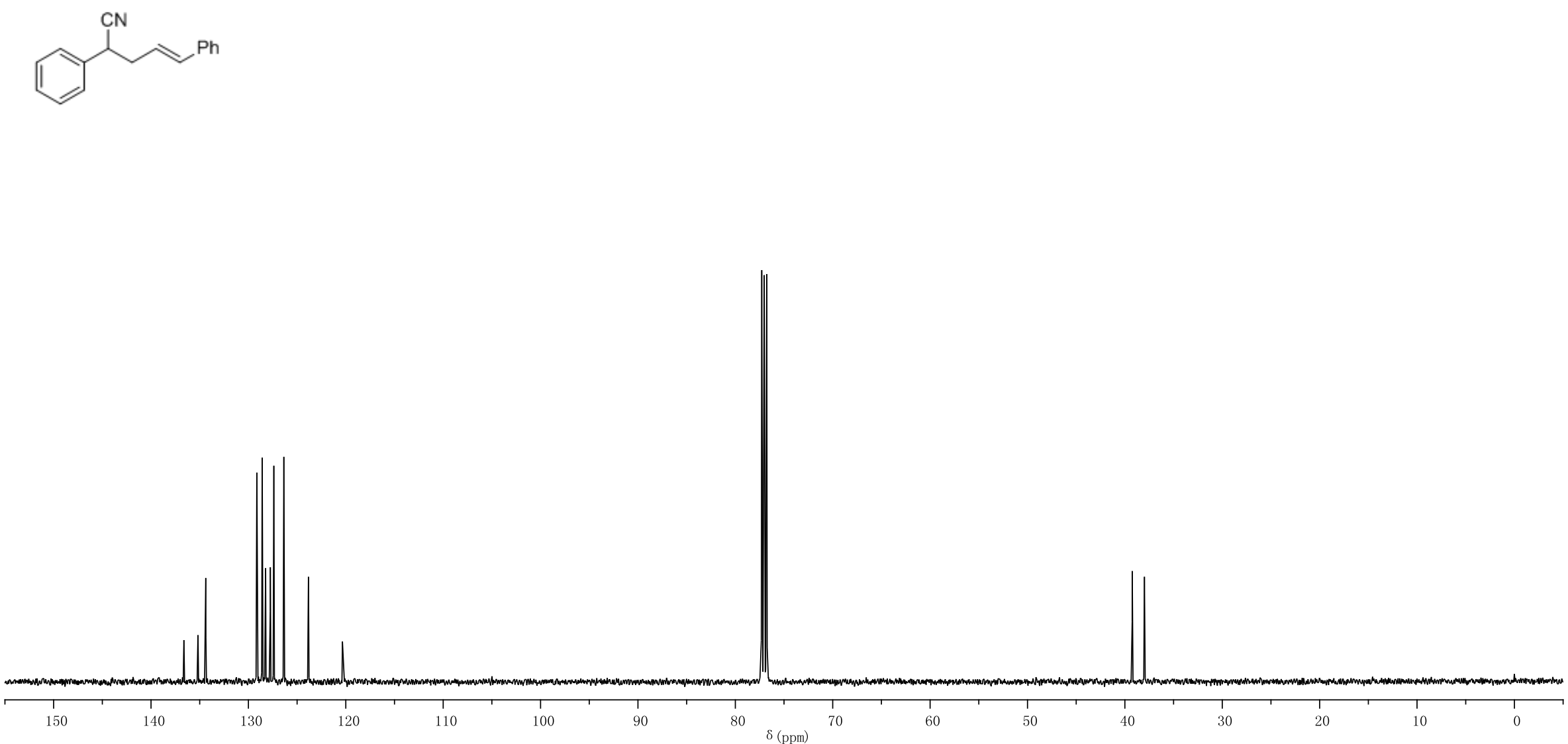


\section{Compound 1x}

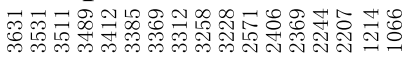

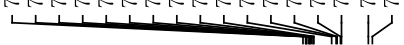

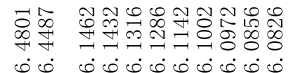

is 000000000

114

${ }^{1} \mathrm{H}$ NMR

$500 \mathrm{MHz}, \mathrm{CDCl}_{3}$
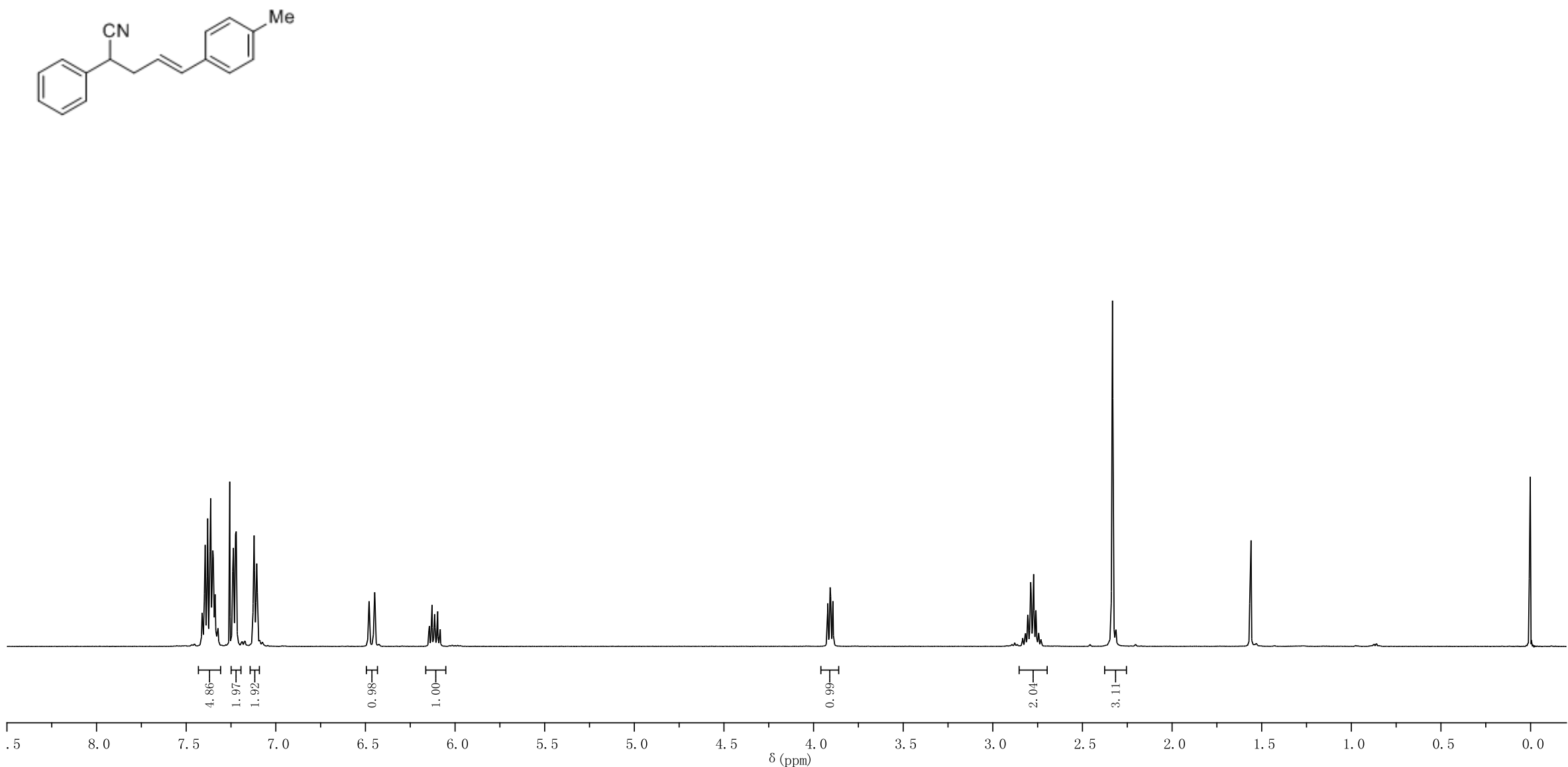


\section{Compound 1x}

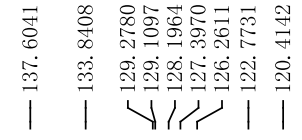

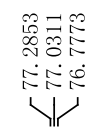

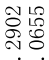

i

${ }^{13} \mathrm{C}\left\{{ }^{1} \mathrm{H}\right\} \mathrm{NMR}$

$125 \mathrm{MHz}, \mathrm{CDCl}_{3}$
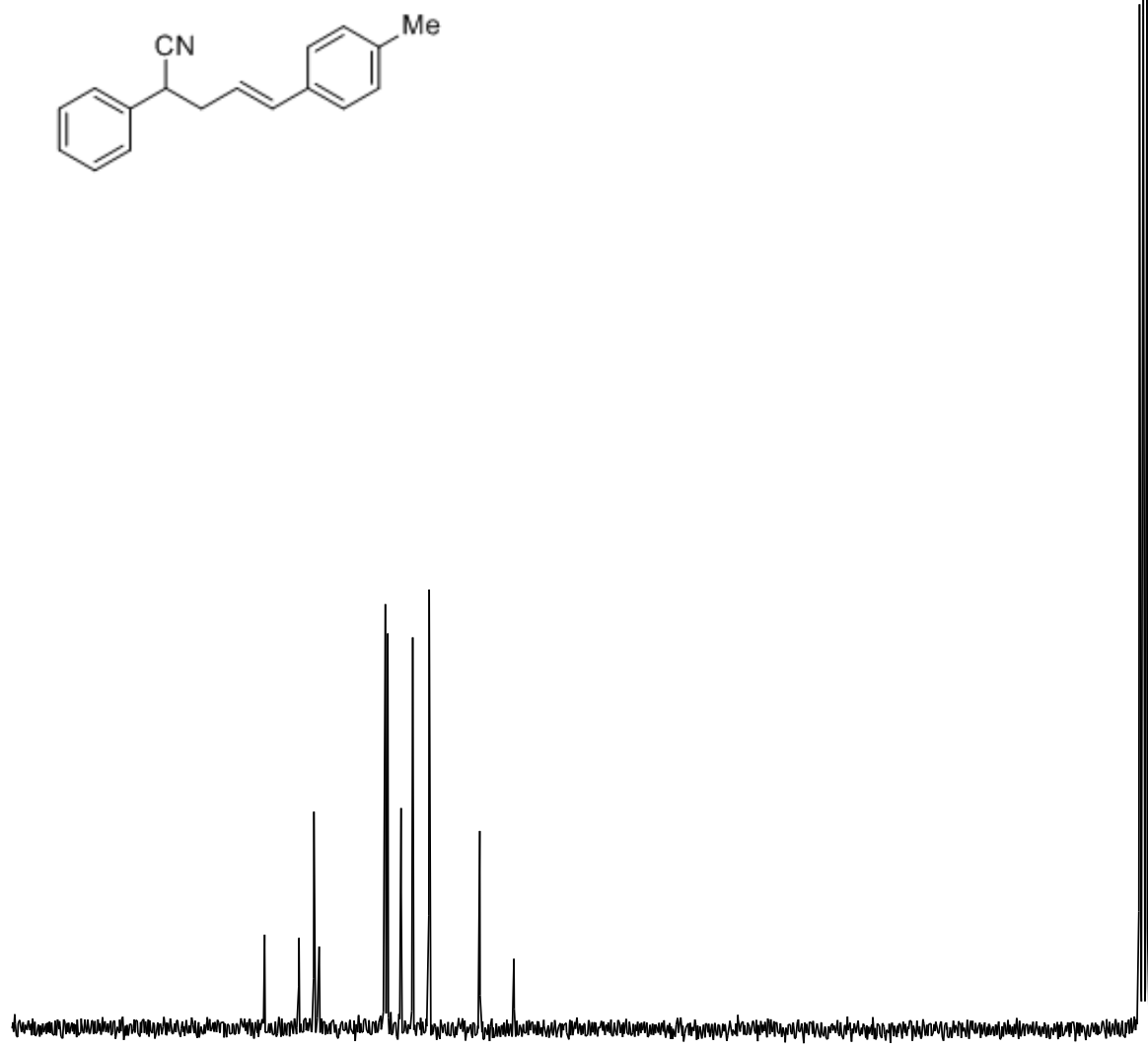

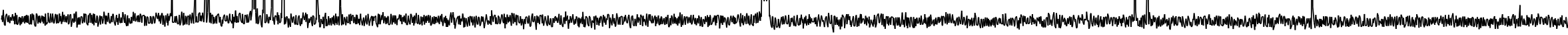

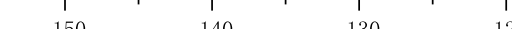

120

10

100

90

80

70

60

50

40

30

20 


\section{Compound 1y}

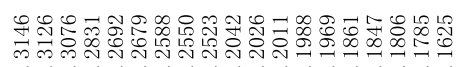

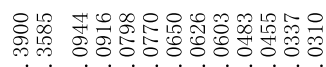

11000000000

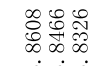

نे $^{\infty}$

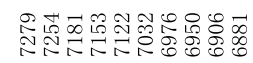

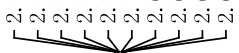

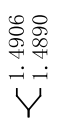

${ }^{1} \mathrm{H} N M R$

$500 \mathrm{MHz}, \mathrm{CDCl}_{3}$
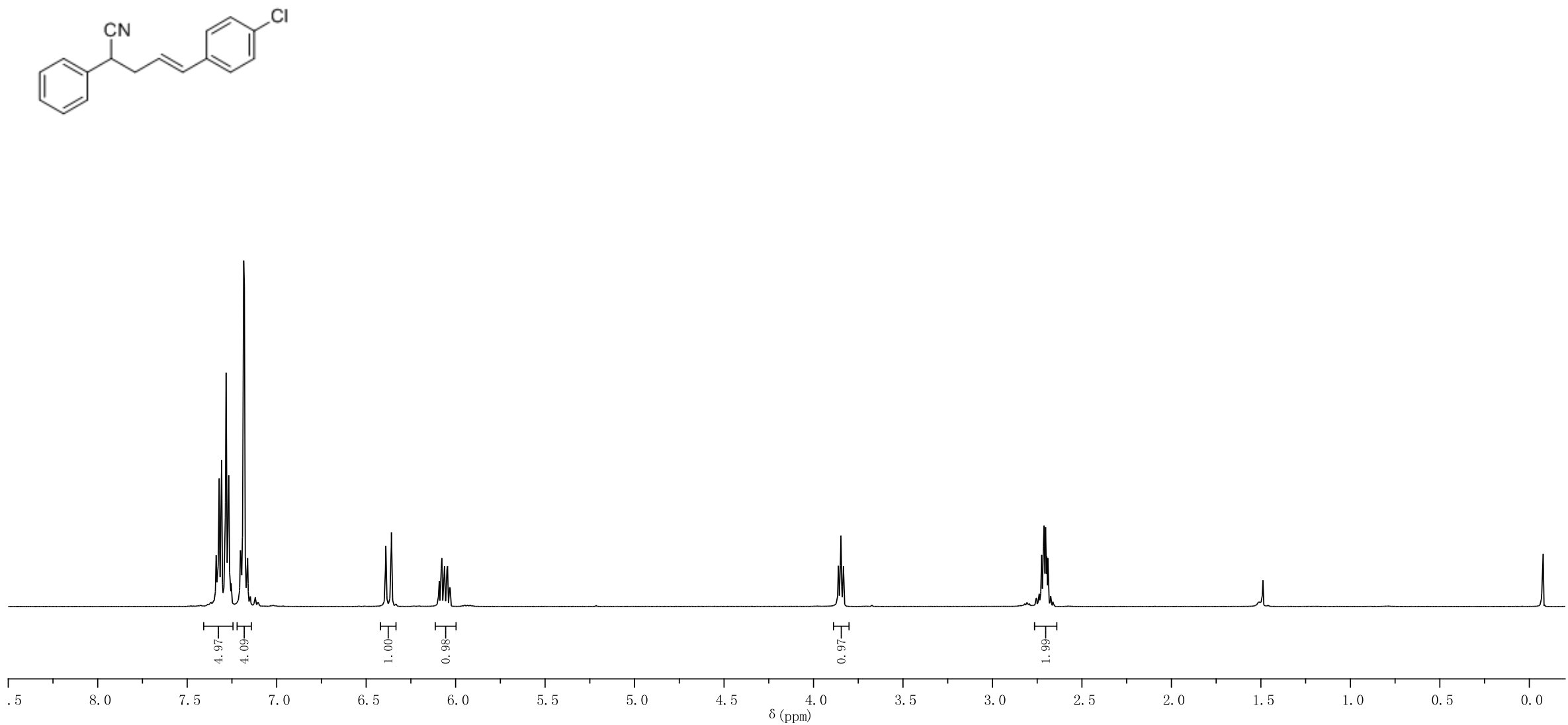
Compound 1y

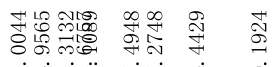

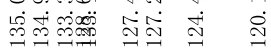

${ }^{13} \mathrm{C}\left\{{ }^{1} \mathrm{H}\right\}$ NMR $125 \mathrm{MHz}, \mathrm{CDCl}_{3}$
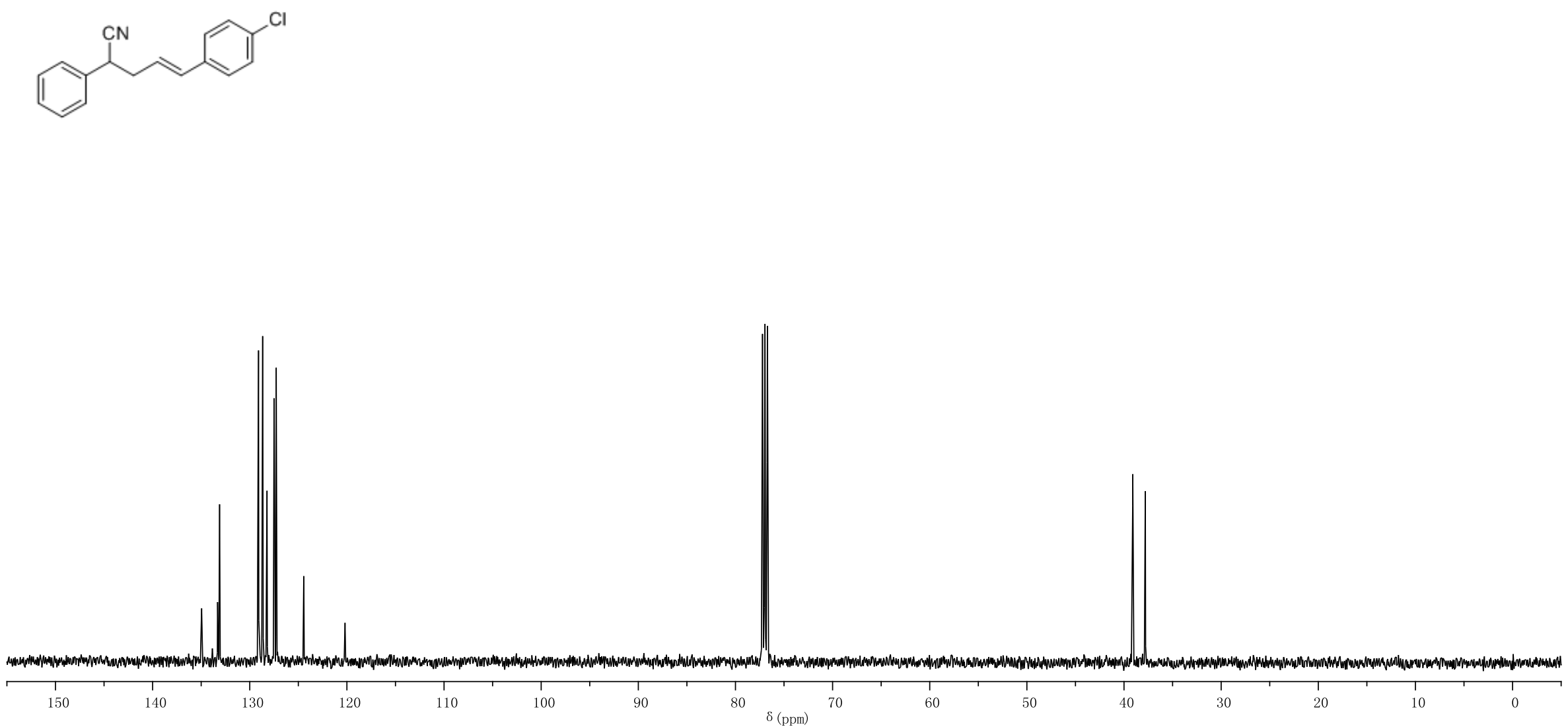


\section{Compound 1z}

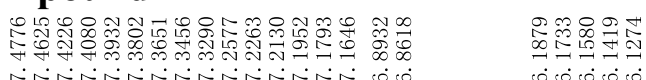

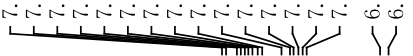

\section{${ }^{1} \mathrm{H}$ NMR}

$500 \mathrm{MHz}, \mathrm{CDCl}_{3}$
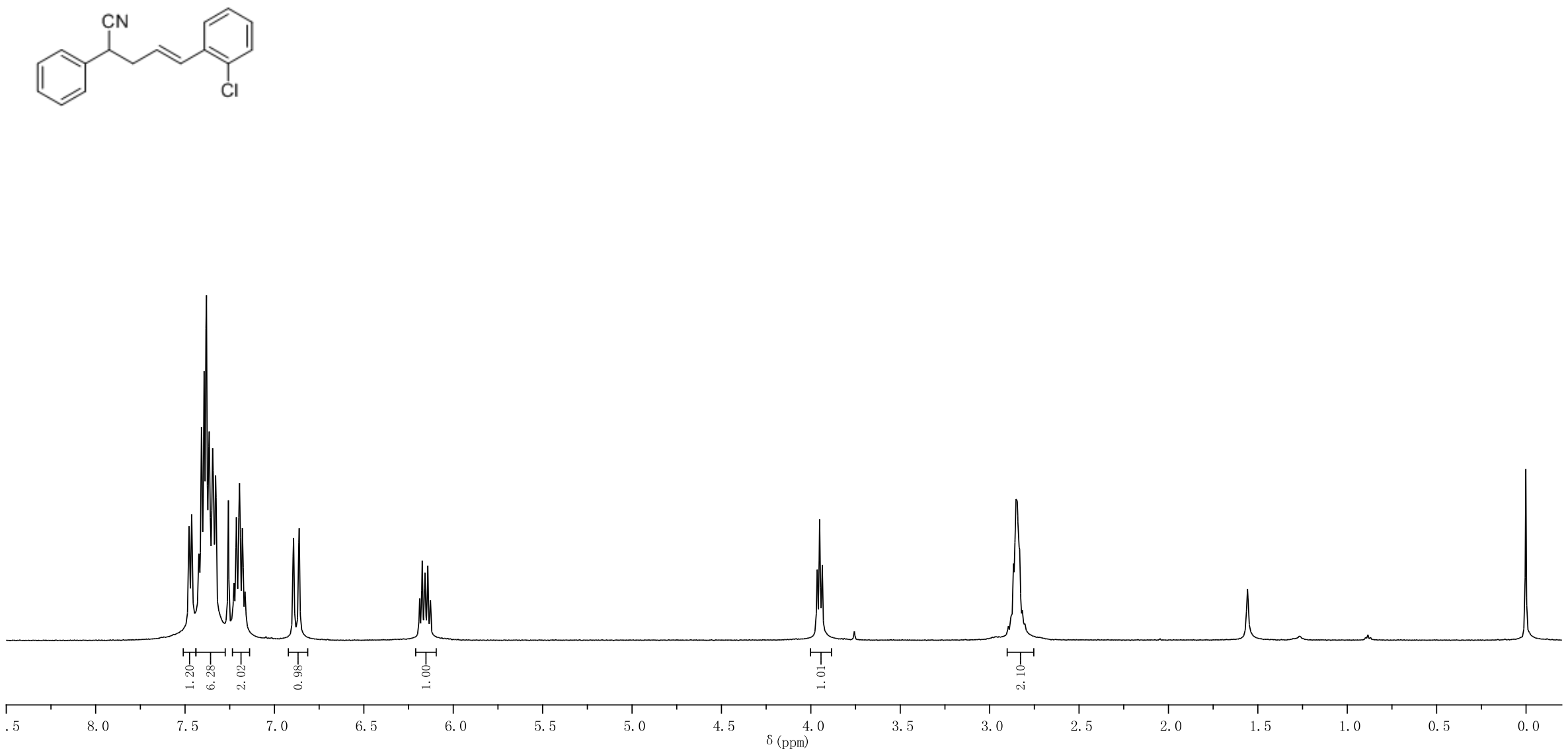
Compound 1z

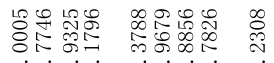

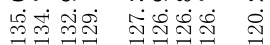

$$
\begin{aligned}
& \text { 化传। }
\end{aligned}
$$

${ }^{13} \mathrm{C}\left\{{ }^{1} \mathrm{H}\right\}$ NMR

$125 \mathrm{MHz}, \mathrm{CDCl}_{3}$

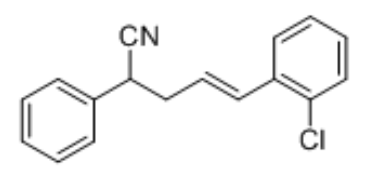

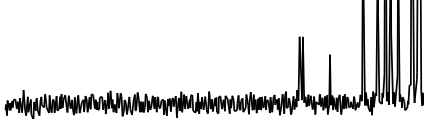

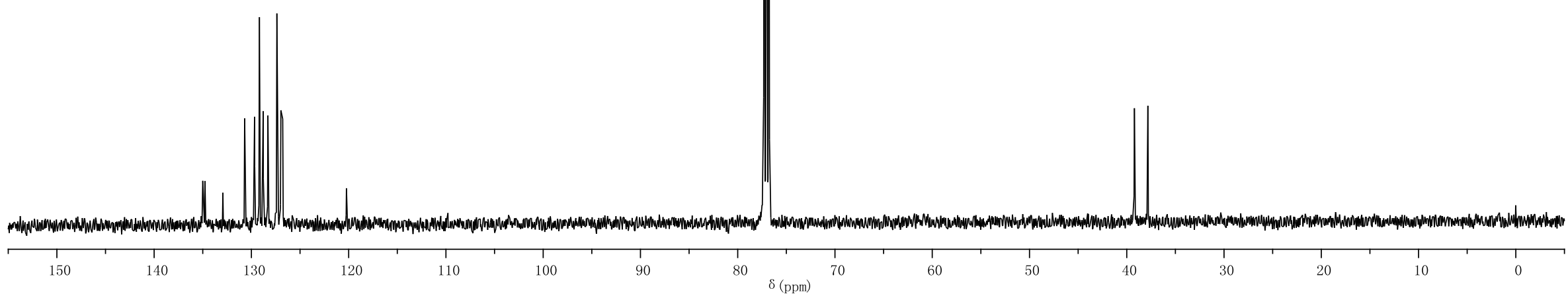




\section{Compound 1za}

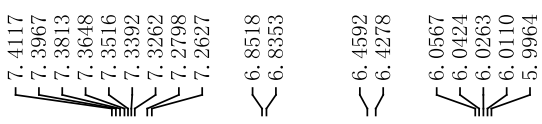

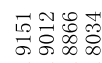

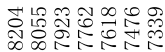

${ }^{1} \mathrm{H}$ NMR

$500 \mathrm{MHz}, \mathrm{CDCl}_{3}$
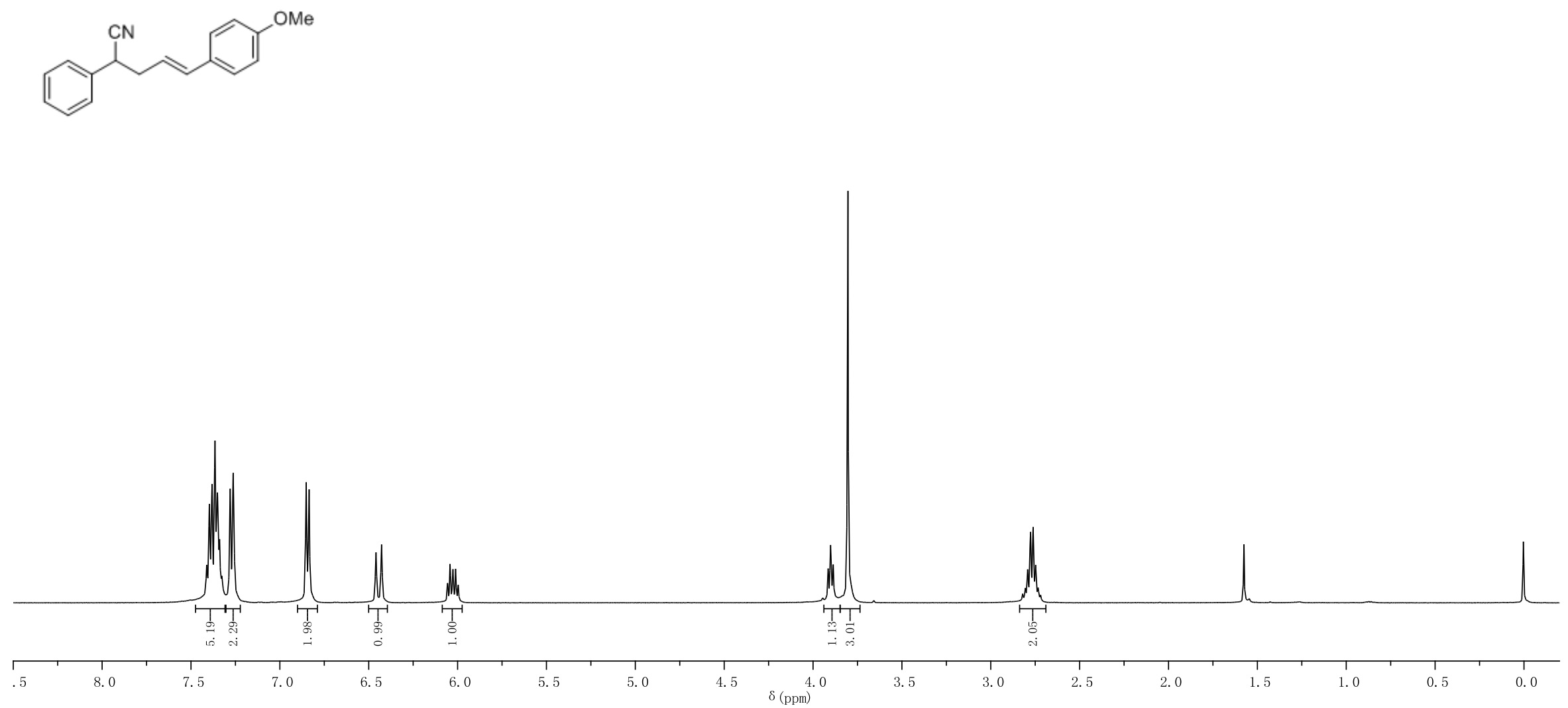
Compound 1za

స్ำ

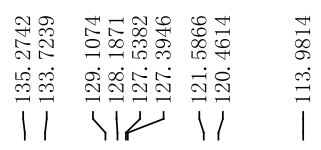

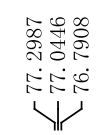

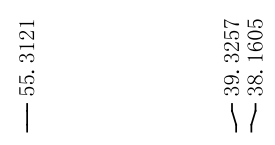

${ }^{13} \mathrm{C}\left\{{ }^{1} \mathrm{H}\right\}$ NMR

$125 \mathrm{MHz}, \mathrm{CDCl}_{3}$
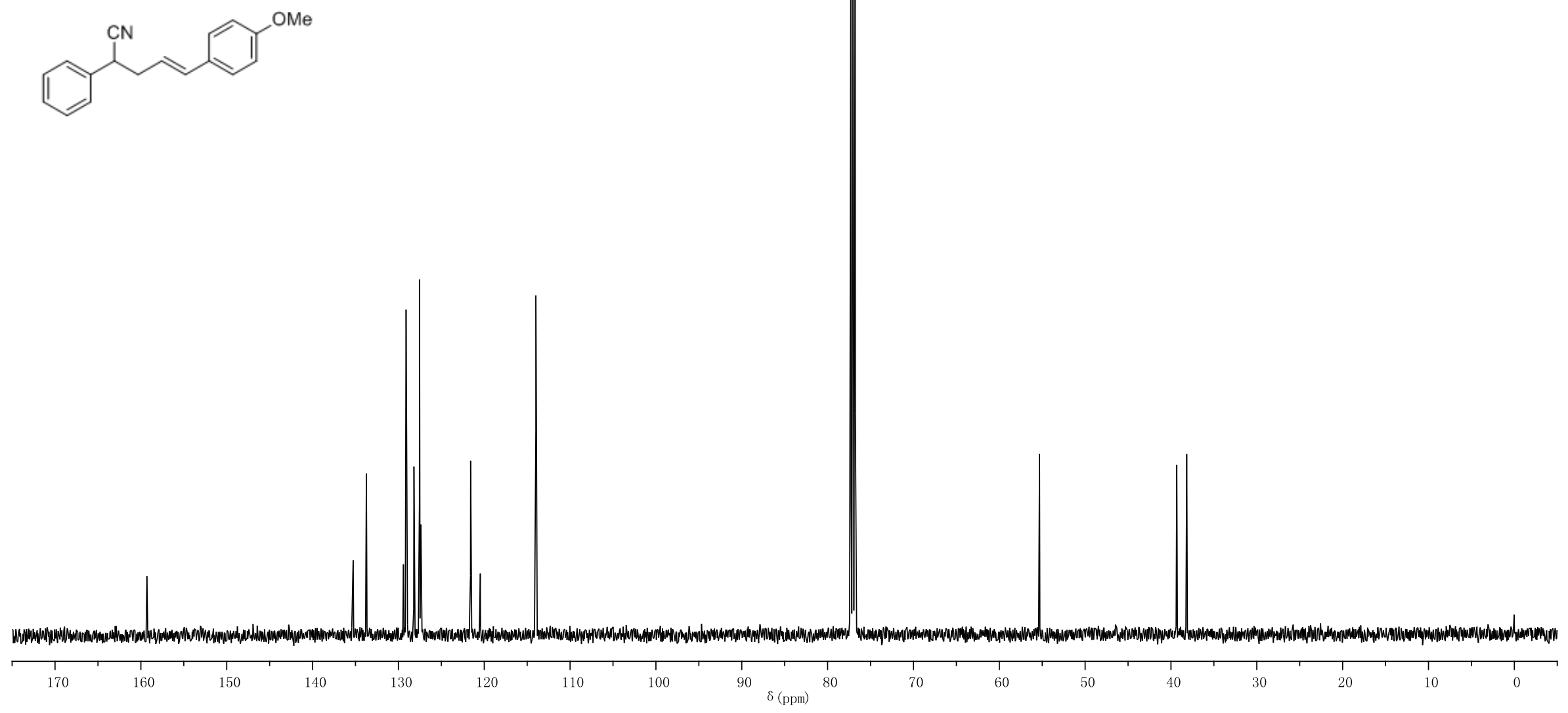

S68 


\section{Compound 1zb}

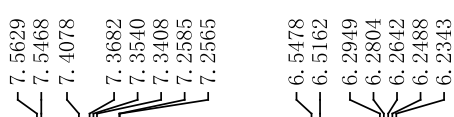

${ }^{1} \mathrm{H}$ NMR

$500 \mathrm{MHz}, \mathrm{CDCl}_{3}$
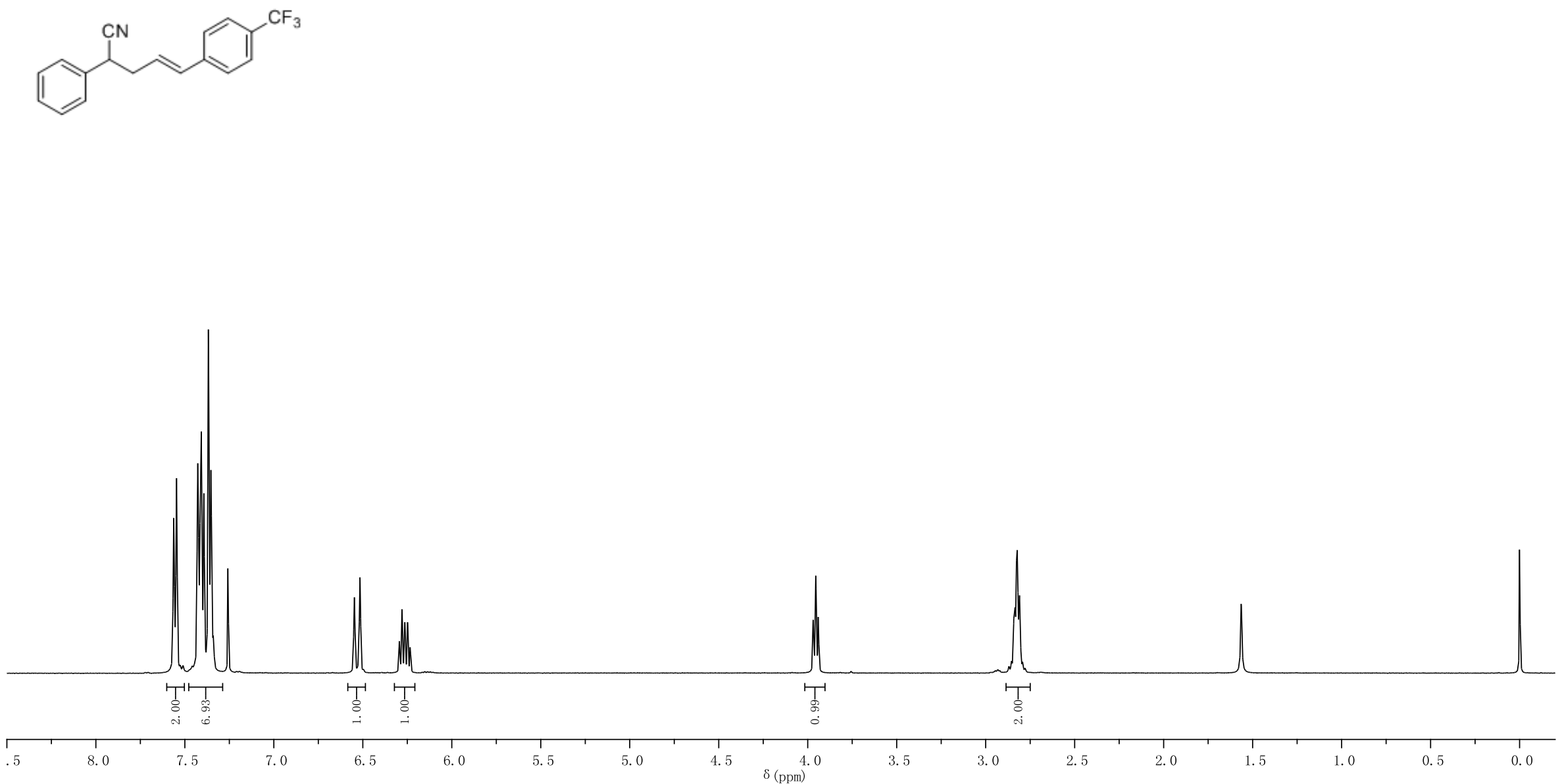


\section{Compound 1zb}

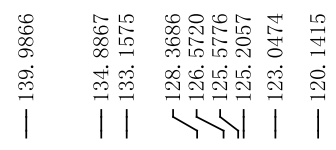

${ }^{13} \mathrm{C}\left\{{ }^{1} \mathrm{H}\right\}$ NMR

$125 \mathrm{MHz}, \mathrm{CDCl}_{3}$
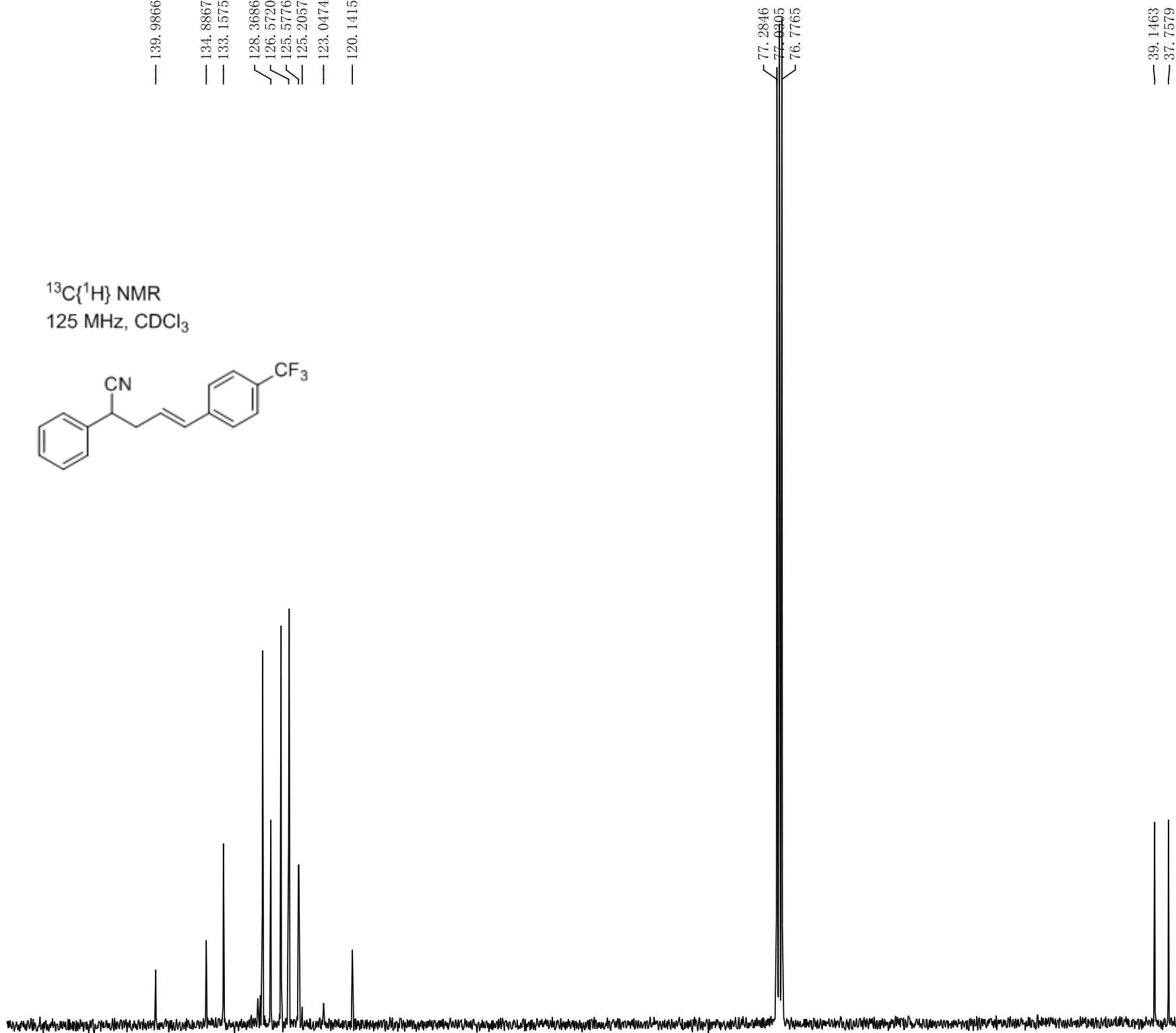

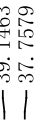

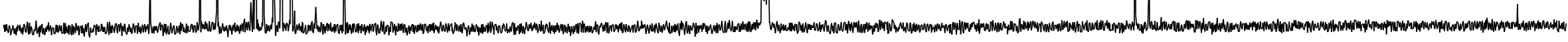

150

140

120

100

90

80

70

60

50

40

30

20

10 


\section{Compound 1zc}

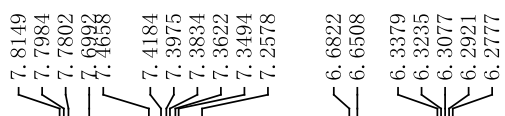

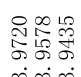

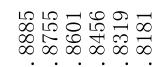

4

${ }^{1} \mathrm{H}$ NMR

$500 \mathrm{MHz}, \mathrm{CDCl}_{3}$
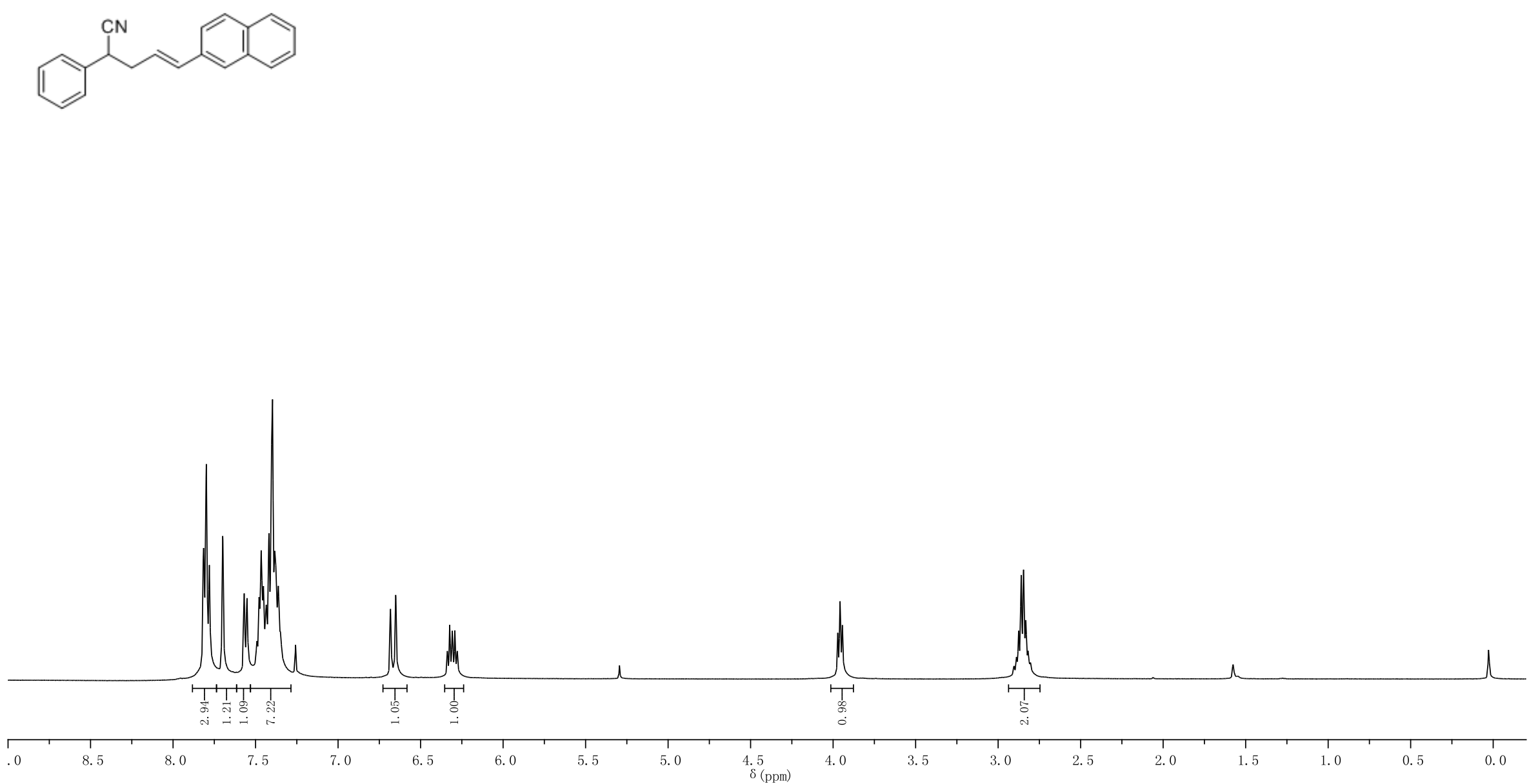
Compound 1zc

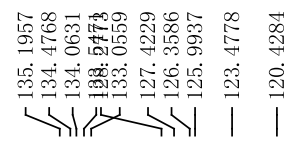

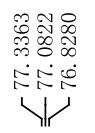

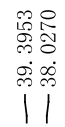

${ }^{13} \mathrm{C}\left\{{ }^{1} \mathrm{H}\right\}$ NMR

$125 \mathrm{MHz}, \mathrm{CDCl}_{3}$
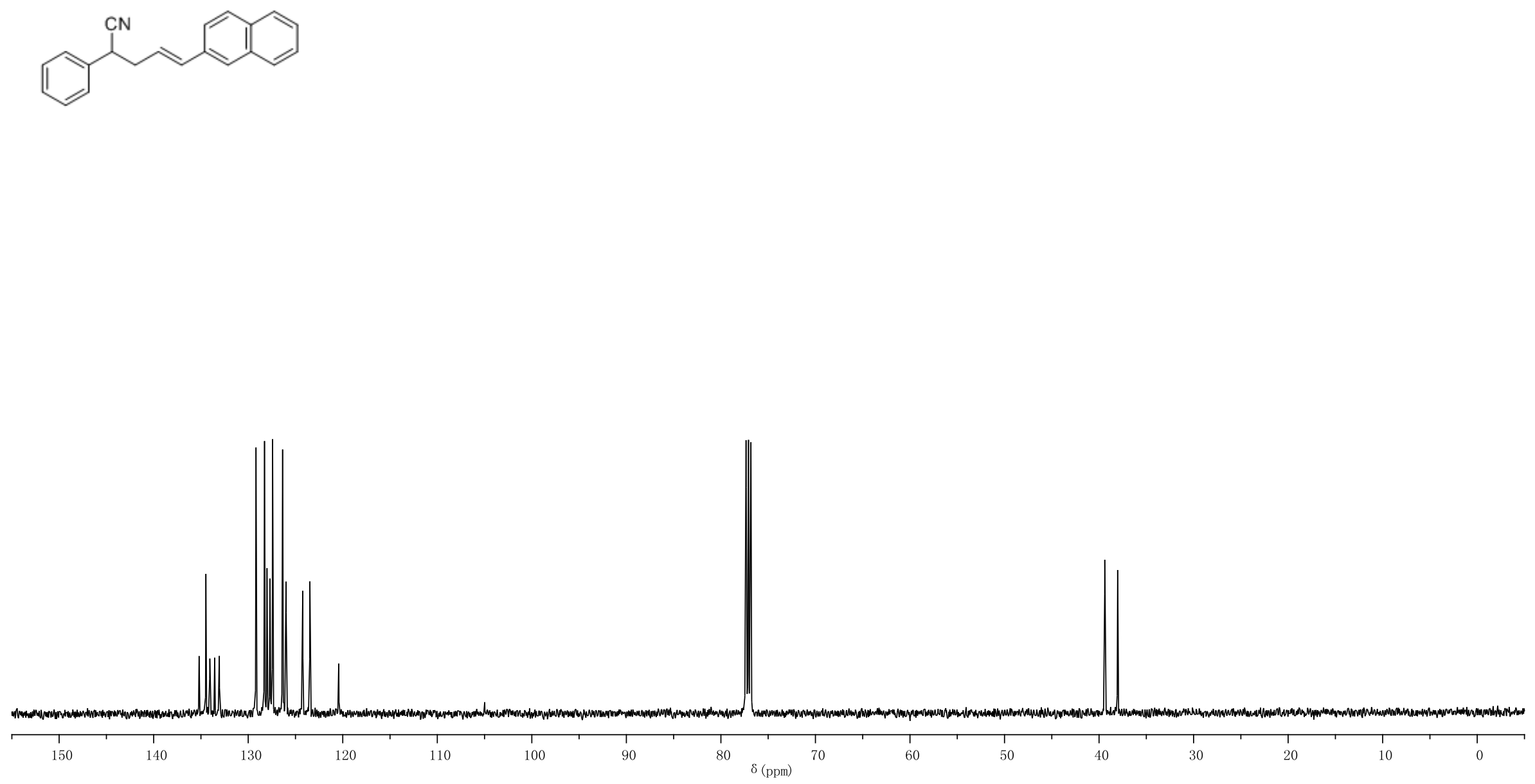

S72 


\section{Compound 1zd}

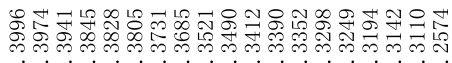

Nisisinisistisin

${ }^{1} \mathrm{H}$ NMR

$500 \mathrm{MHz}, \mathrm{CDCl}_{3}$
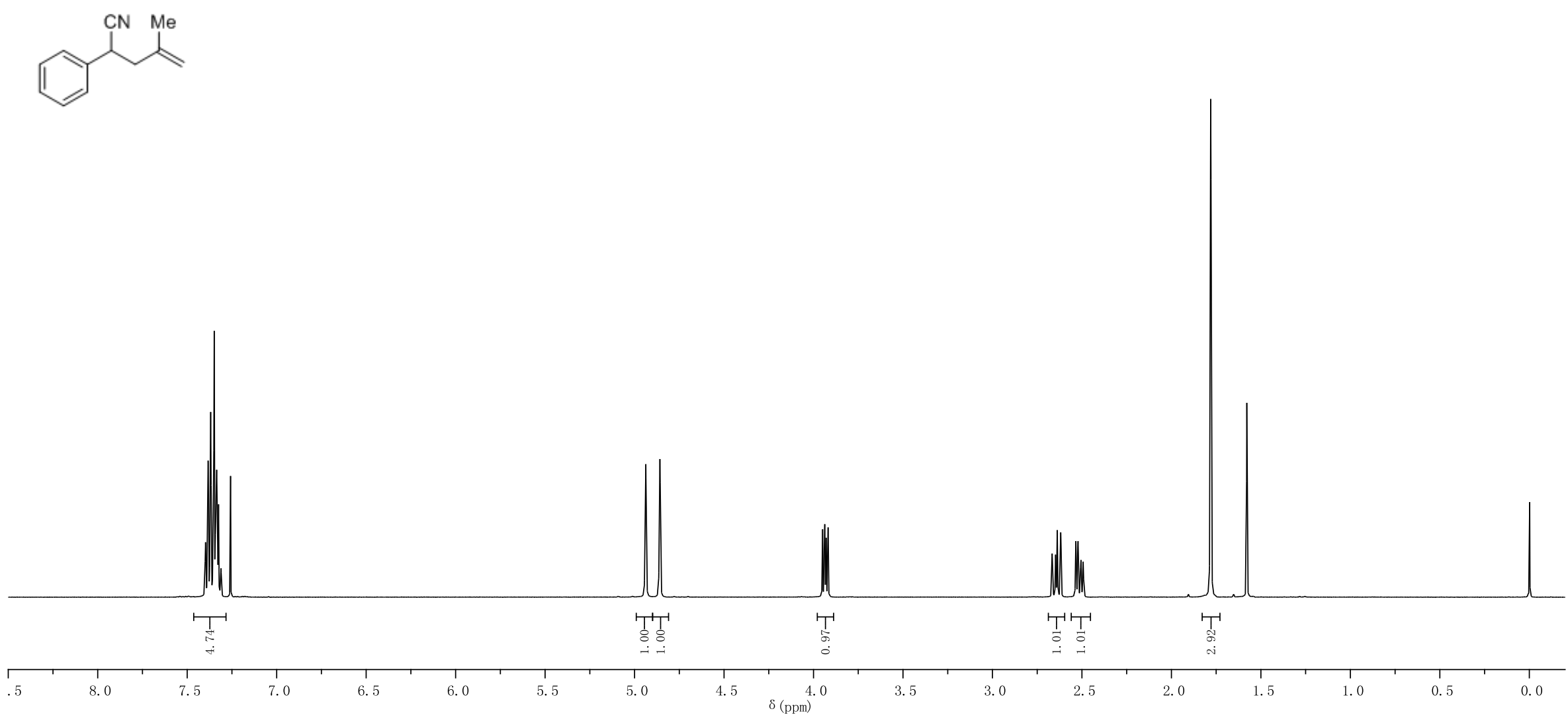
Compound 1zd

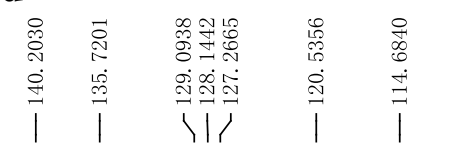

${ }^{13} \mathrm{C}\left\{{ }^{1} \mathrm{H}\right\}$ NMR

$125 \mathrm{MHz}, \mathrm{CDCl}_{3}$
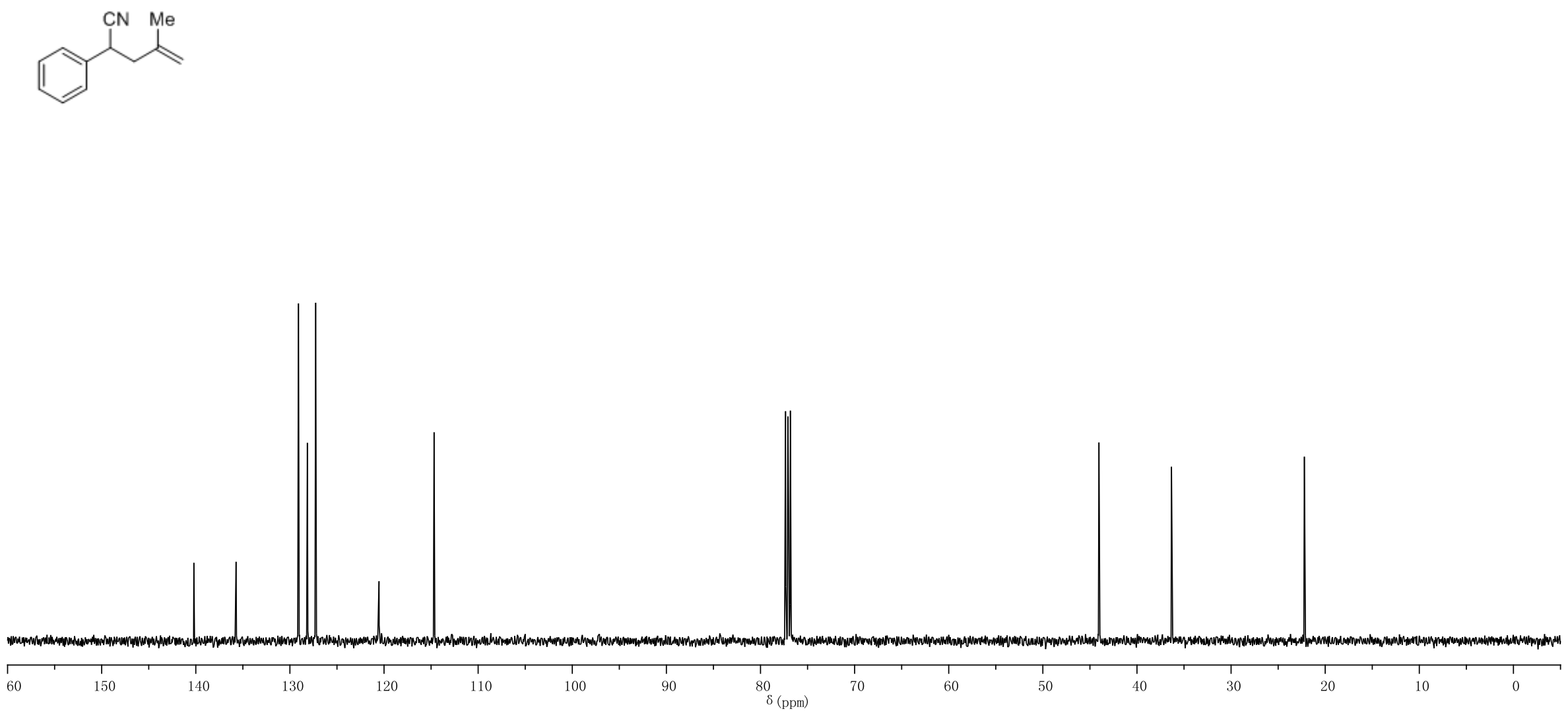


\section{Compound 1ze}

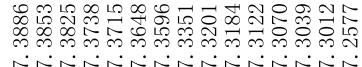

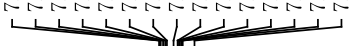

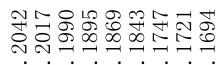

is

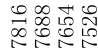

עim

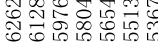

Ninini

$\stackrel{\infty}{\stackrel{\infty}{\infty}}$

$\dot{1}$

${ }^{1} \mathrm{H}$ NMR

$500 \mathrm{MHz}, \mathrm{CDCl}_{3}$
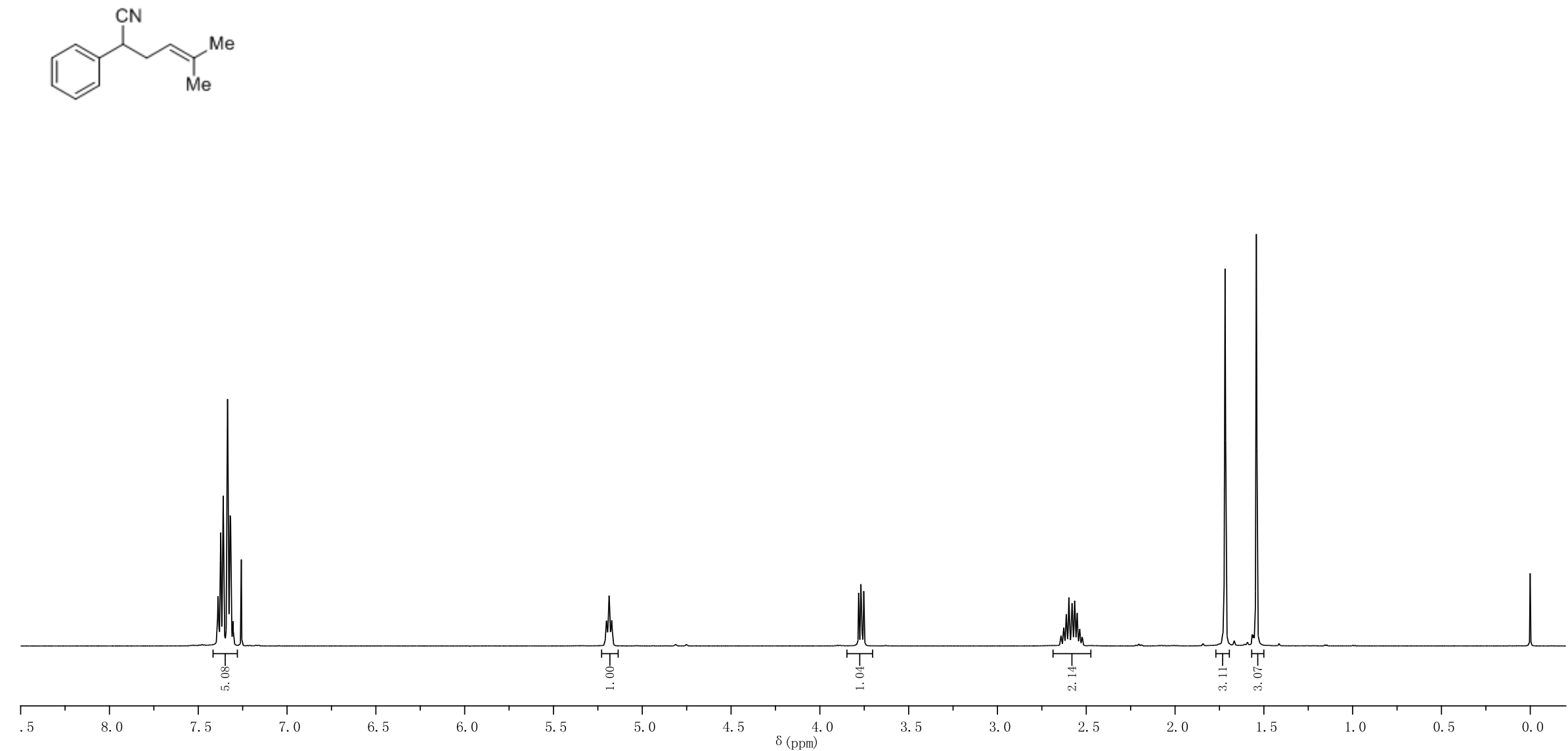


\section{Compound 1ze}

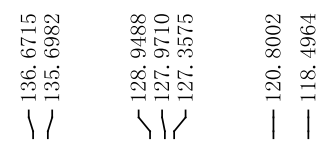

${ }^{13} \mathrm{C}\left\{{ }^{1} \mathrm{H}\right\}$ NMR

$125 \mathrm{MHz}, \mathrm{CDCl}_{3}$
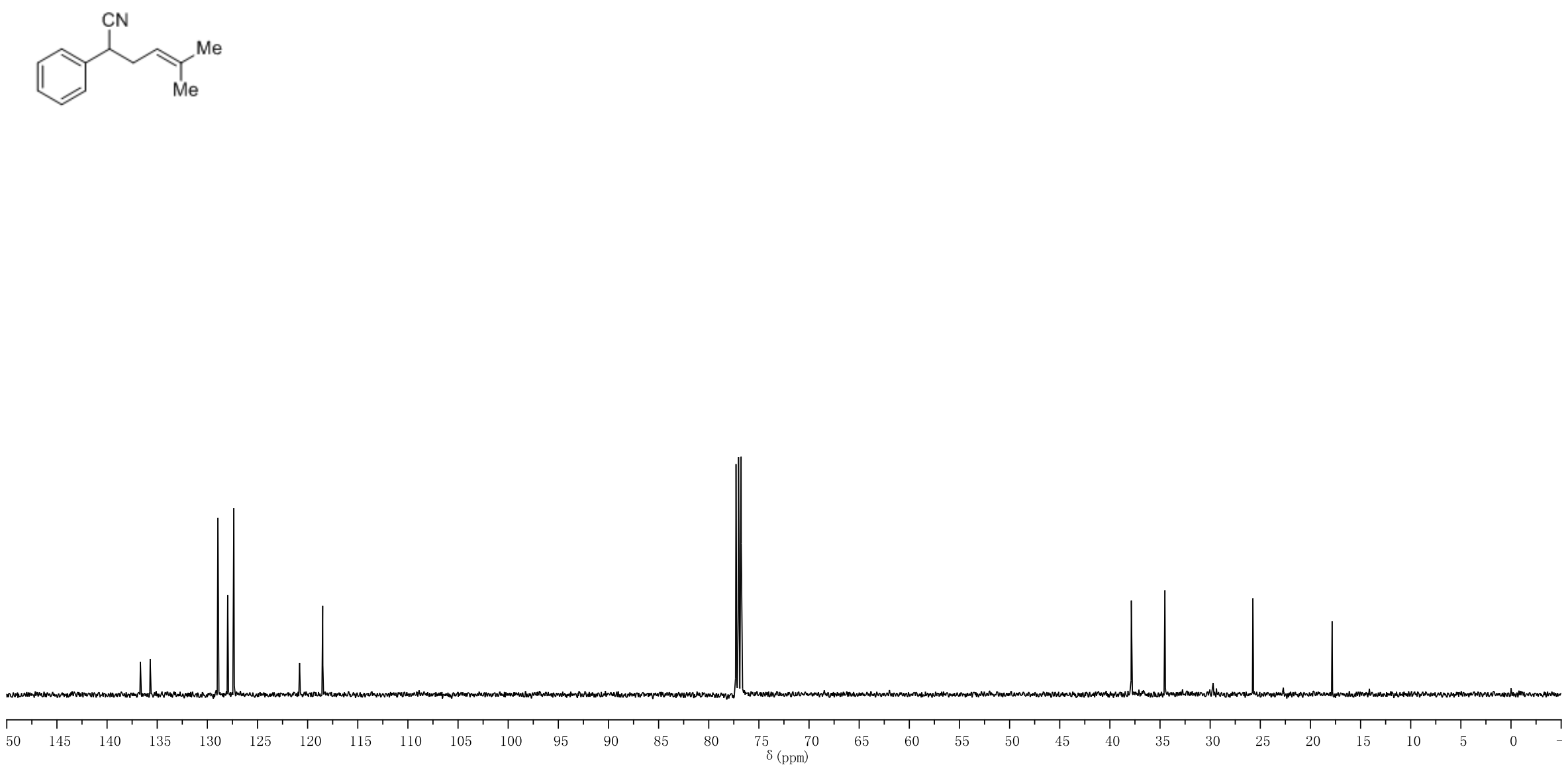


\section{Compound 1zg}

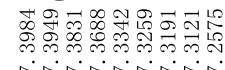

(1)

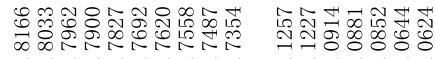

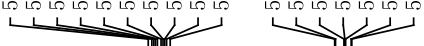

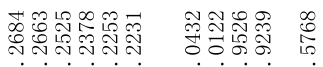

Nindi

${ }^{1} \mathrm{H}$ NMR

$500 \mathrm{MHz}, \mathrm{CDCl}_{3}$
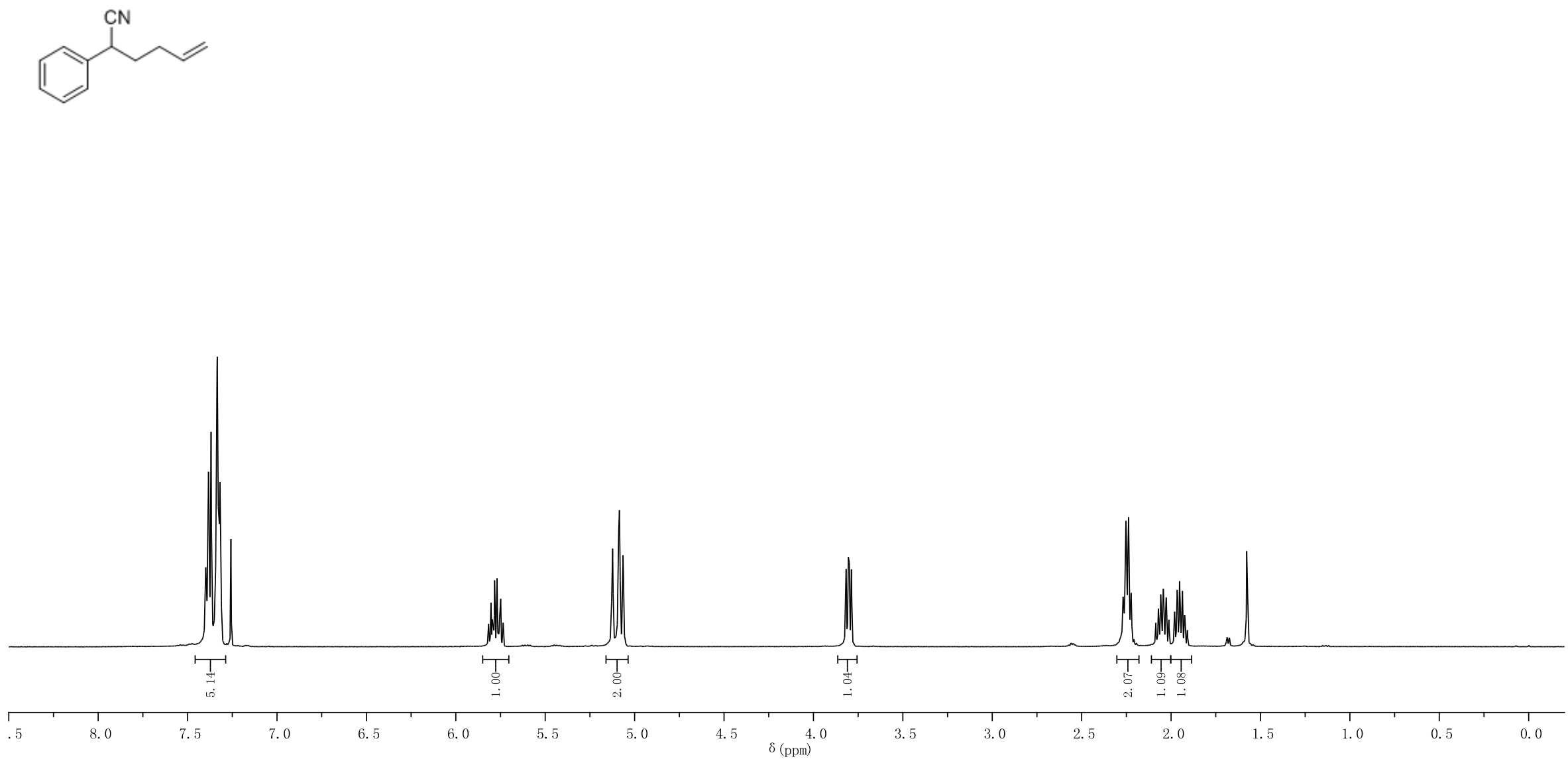


\section{Compound 1zg}

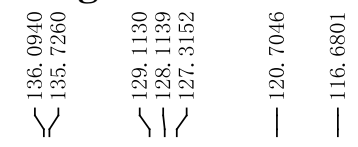

${ }^{13} \mathrm{C}\left\{{ }^{1} \mathrm{H}\right\}$ NMR

$125 \mathrm{MHz}, \mathrm{CDCl}_{3}$
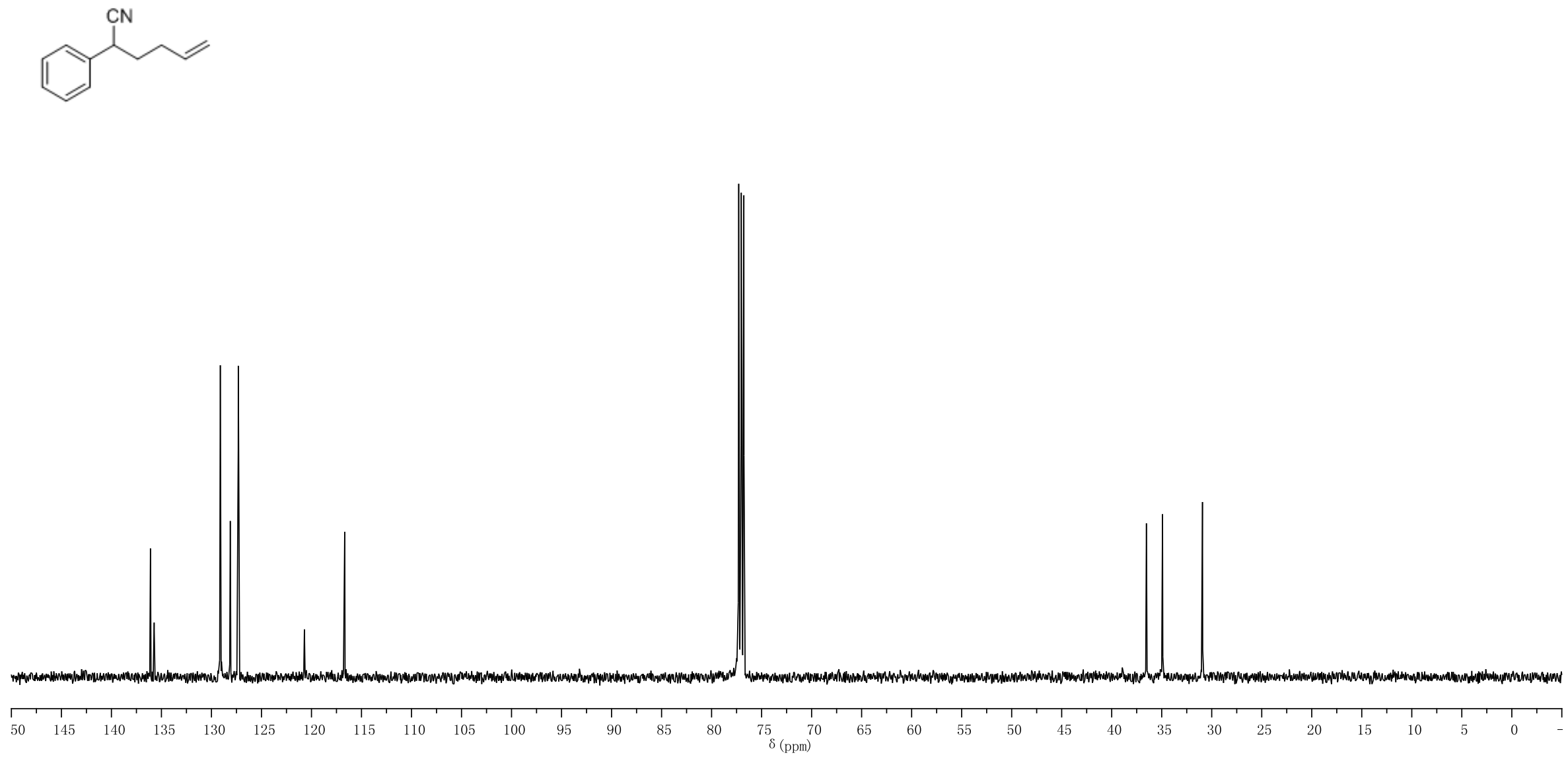


\section{Compound 1zi}

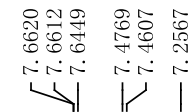

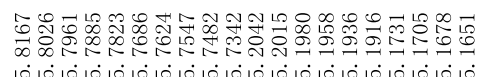

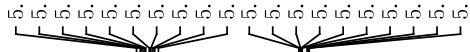

\section{${ }^{1} \mathrm{H}$ NMR}

$500 \mathrm{MHz}, \mathrm{CDCl}_{3}$
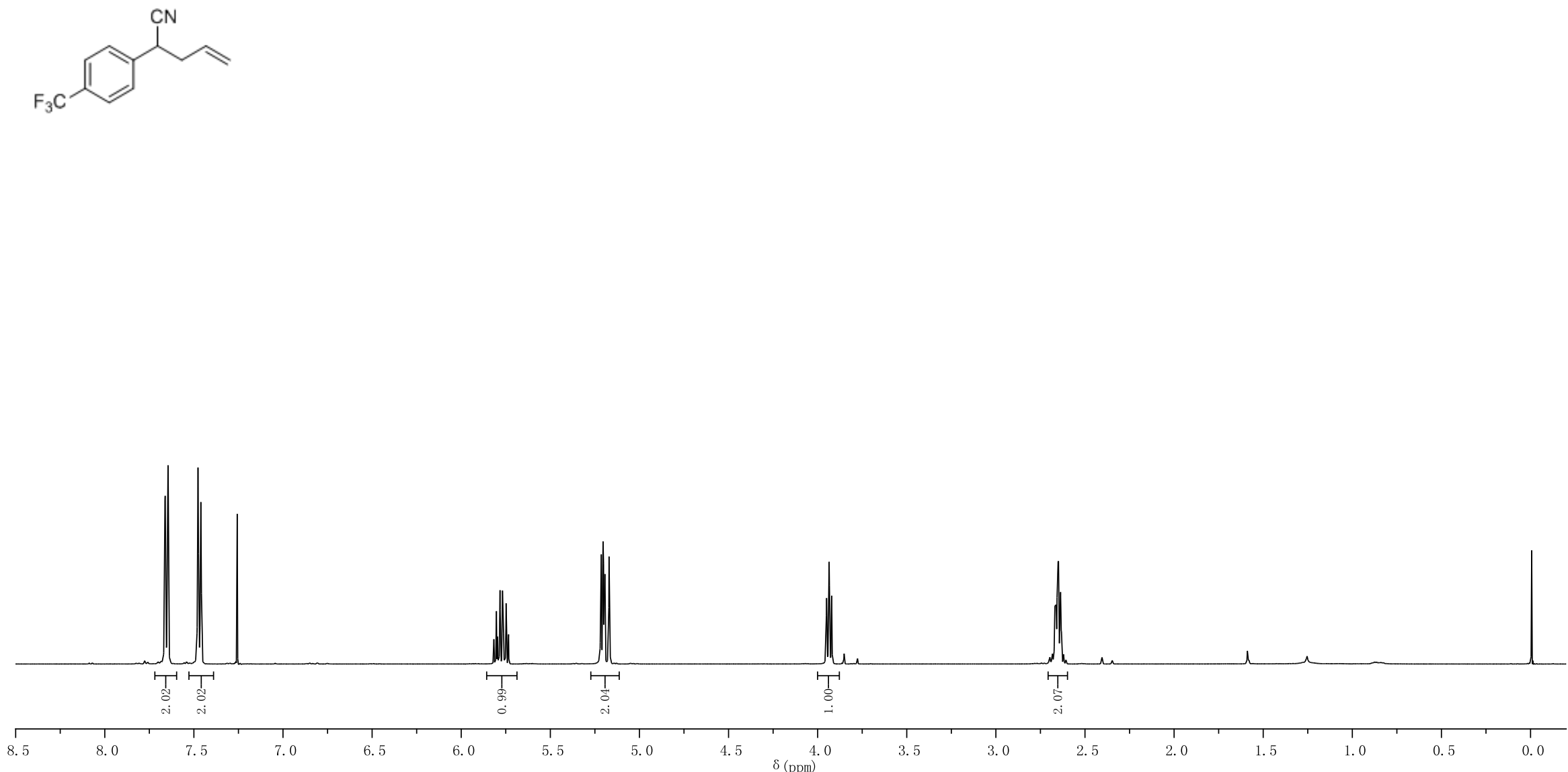
Compound 1zi

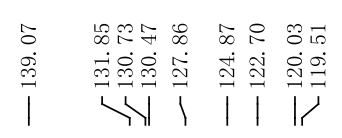

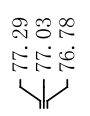

$\mid \begin{aligned} & \infty \\ & \substack{\infty \\ 0} \\ & \substack{0 \\ 0}\end{aligned}$

$\stackrel{8}{8}$

${ }^{13} \mathrm{C}\left\{{ }^{1} \mathrm{H}\right\}$ NMR

$125 \mathrm{MHz}, \mathrm{CDCl}_{3}$

舟

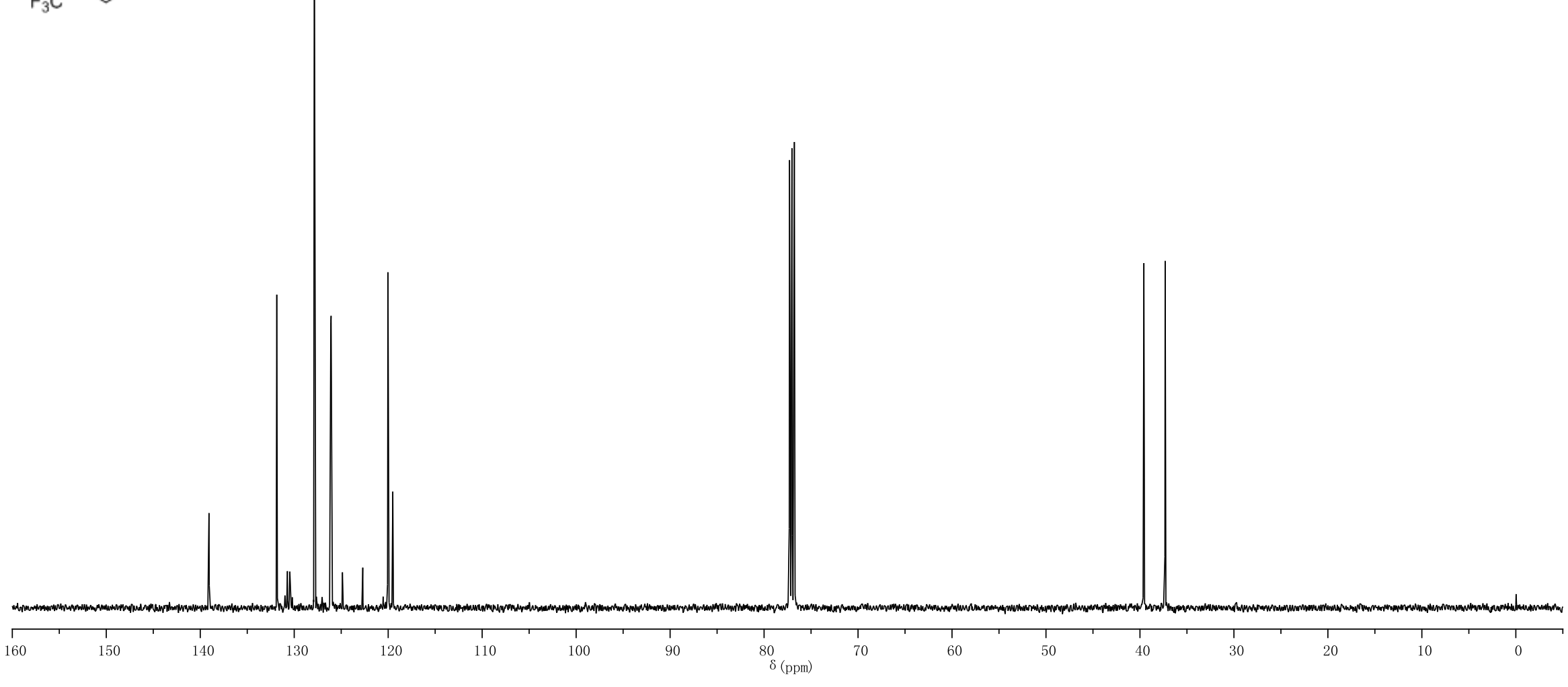

S80 


\section{Compound 1zj}

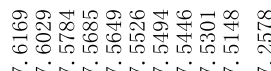

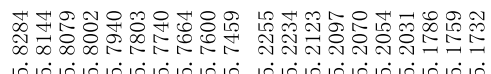

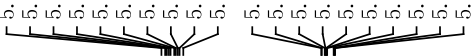

\section{${ }^{1} \mathrm{H}$ NMR}

$500 \mathrm{MHz}, \mathrm{CDCl}_{3}$<smiles>C=CCC(C#N)c1cccc(C(F)(F)F)c1</smiles>

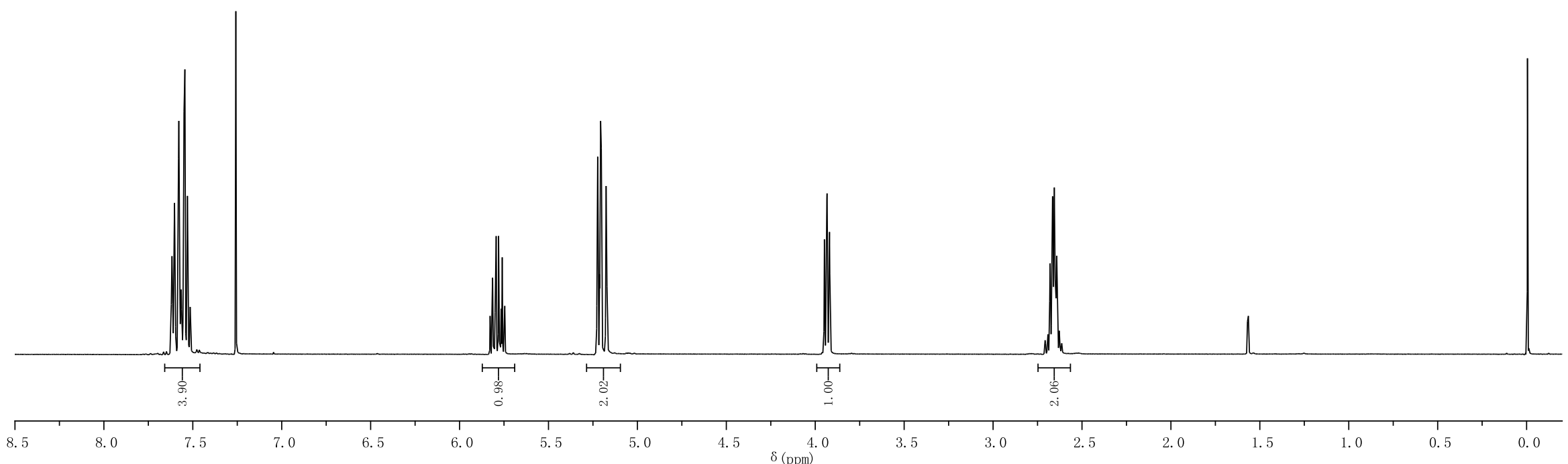




\section{Compound 1zj}

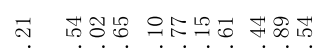

๑

। रोखी।

응ㄴㅇㅇㅇㅛ

余金

낭오

i

${ }^{13} \mathrm{C}\left\{{ }^{1} \mathrm{H}\right\}$ NMR

$125 \mathrm{MHz}, \mathrm{CDCl}_{3}$
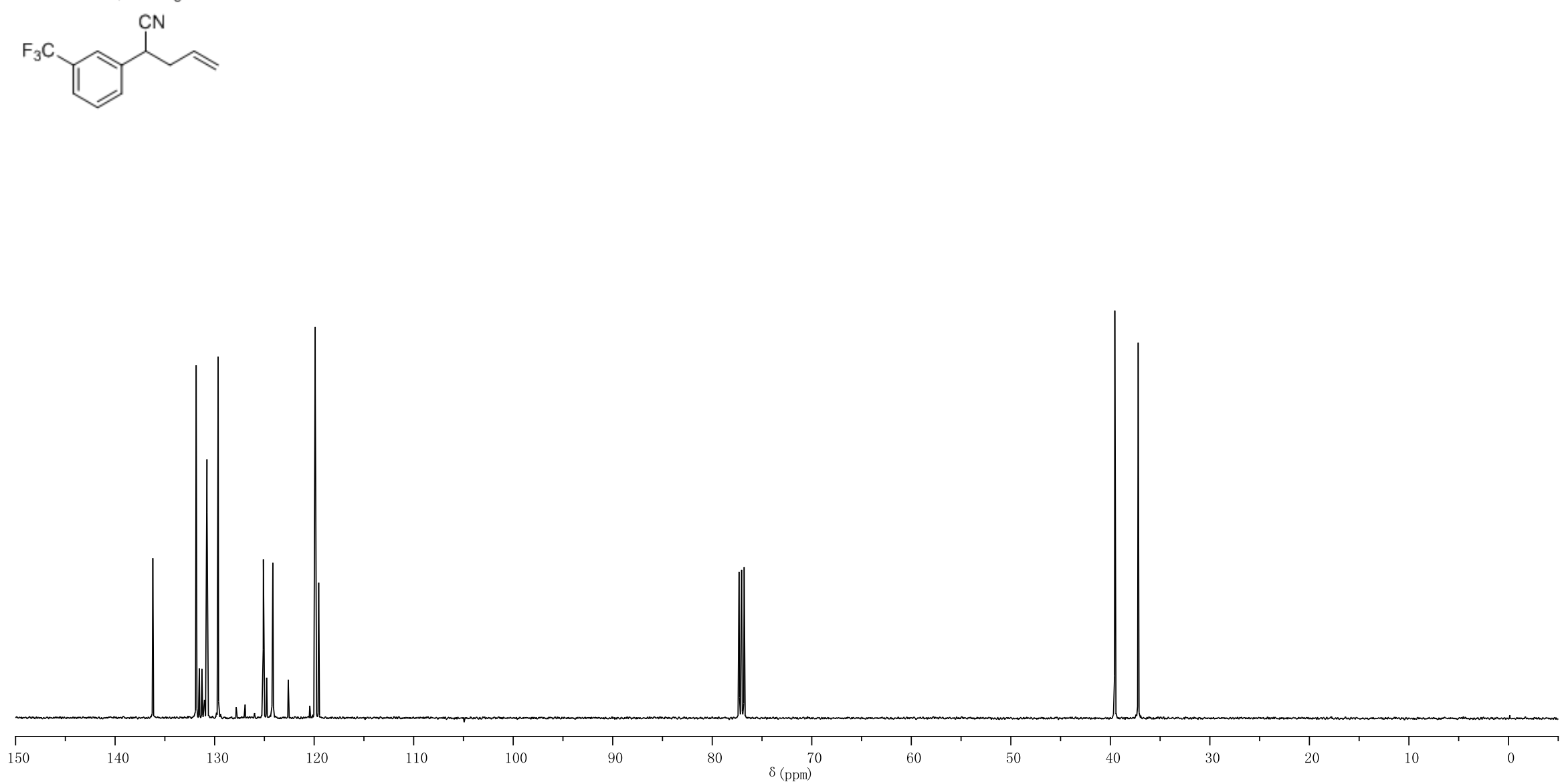


\section{Compound 1zk}

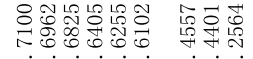 \\ 논 |}
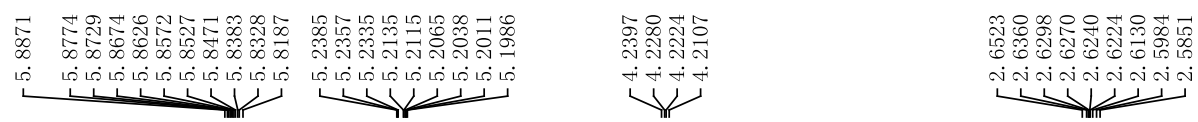

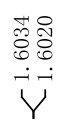

${ }^{1} \mathrm{H}$ NMR

$500 \mathrm{MHz}, \mathrm{CDCl}_{3}$
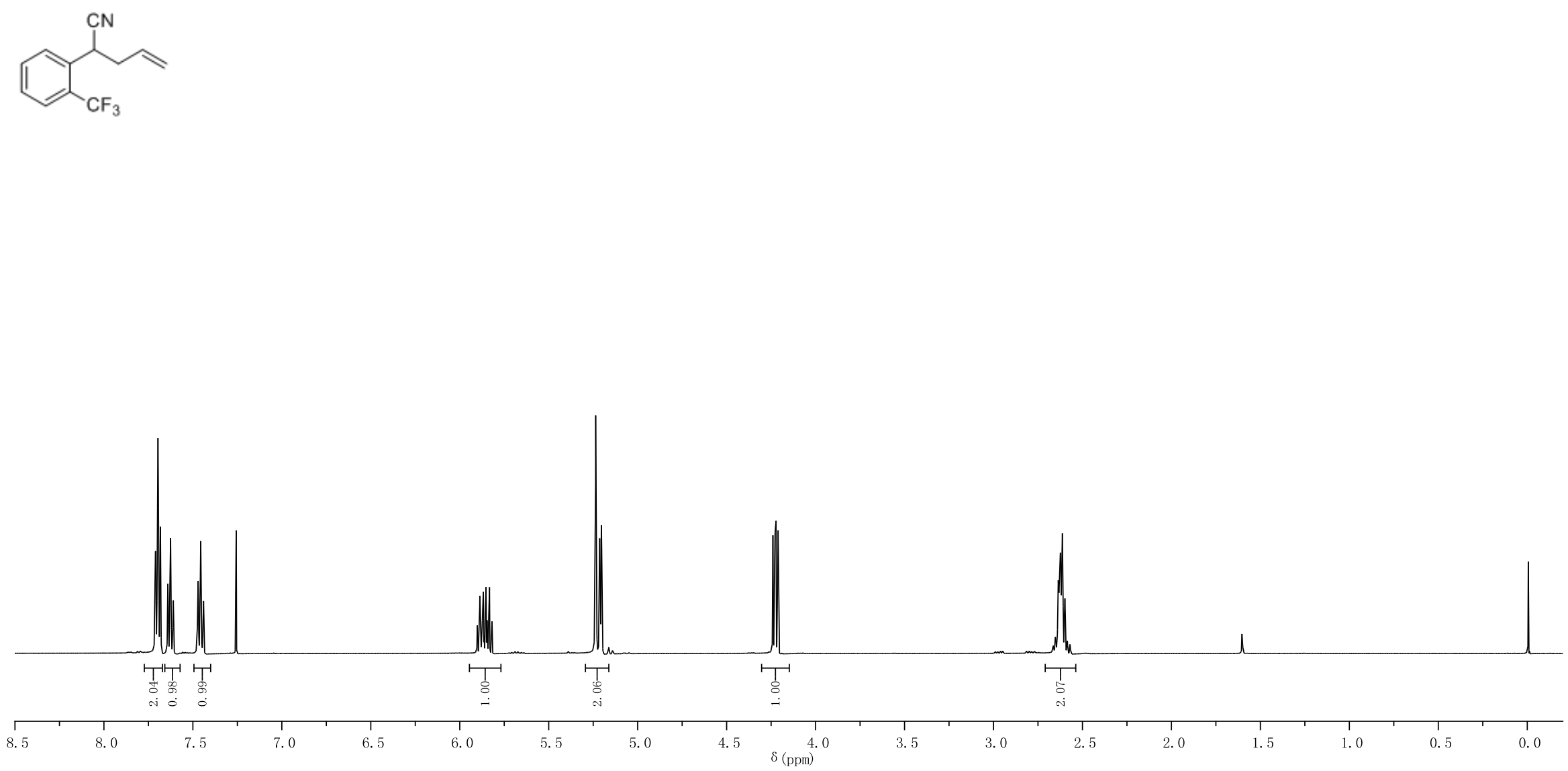


\section{Compound 1zk}

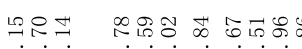

ฝึ่

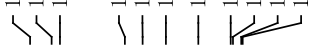

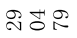

사요

मे ले

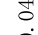

सा H| |

${ }^{13} \mathrm{C}\left\{{ }^{1} \mathrm{H}\right\}$ NMR

$125 \mathrm{MHz}, \mathrm{CDCl}_{3}$
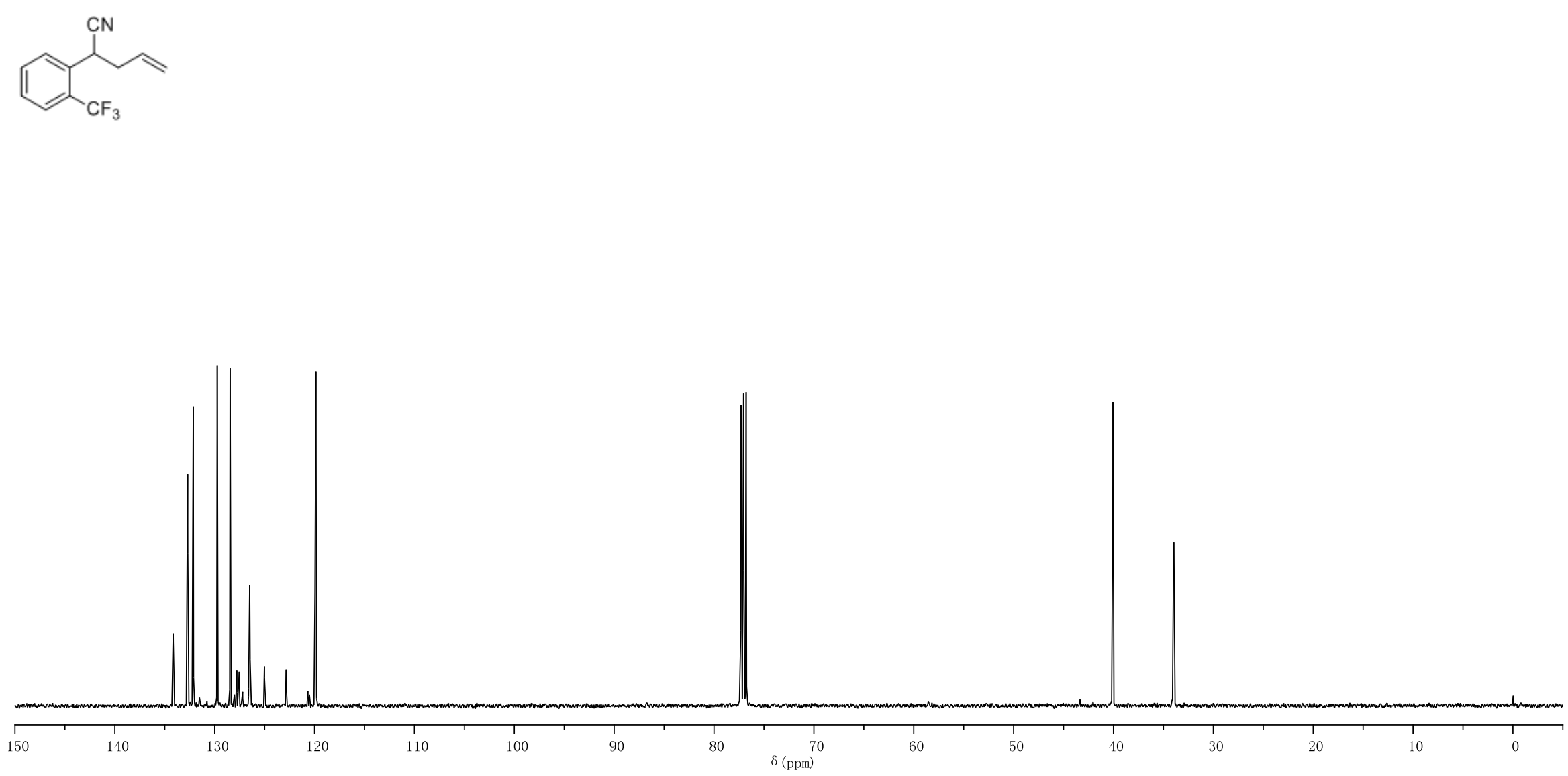


\section{Compound 1zl}

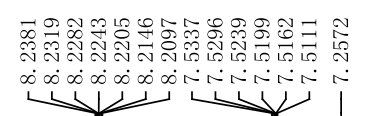

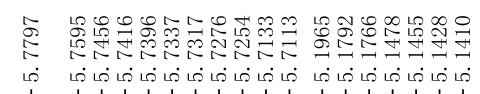

Lم

${ }^{1} \mathrm{H}$ NMR

$500 \mathrm{MHz}, \mathrm{CDCl}_{3}$<smiles>C=CCC(C#N)c1ccc([N+](=O)[O-])cc1</smiles>

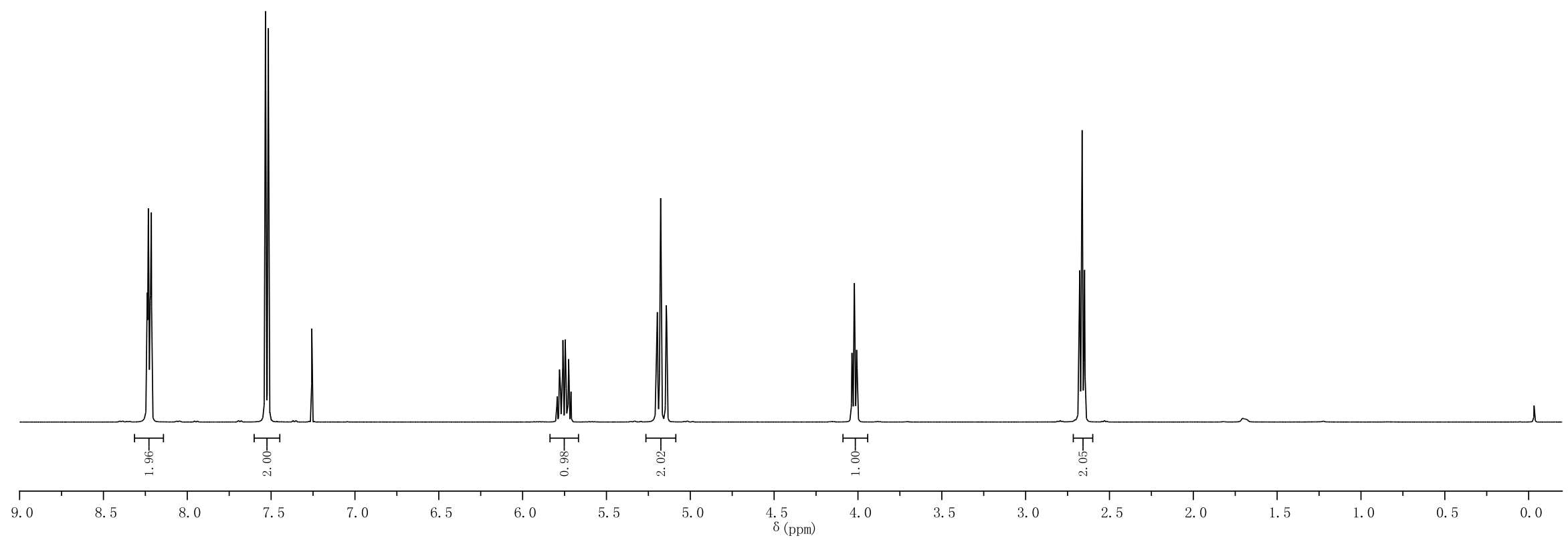




\section{Compound 1zl}

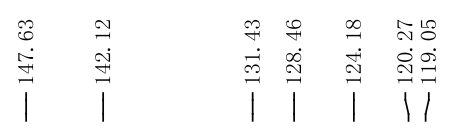

${ }^{13} \mathrm{C}\left\{{ }^{1} \mathrm{H}\right\}$ NMR

$125 \mathrm{MHz}, \mathrm{CDCl}_{3}$

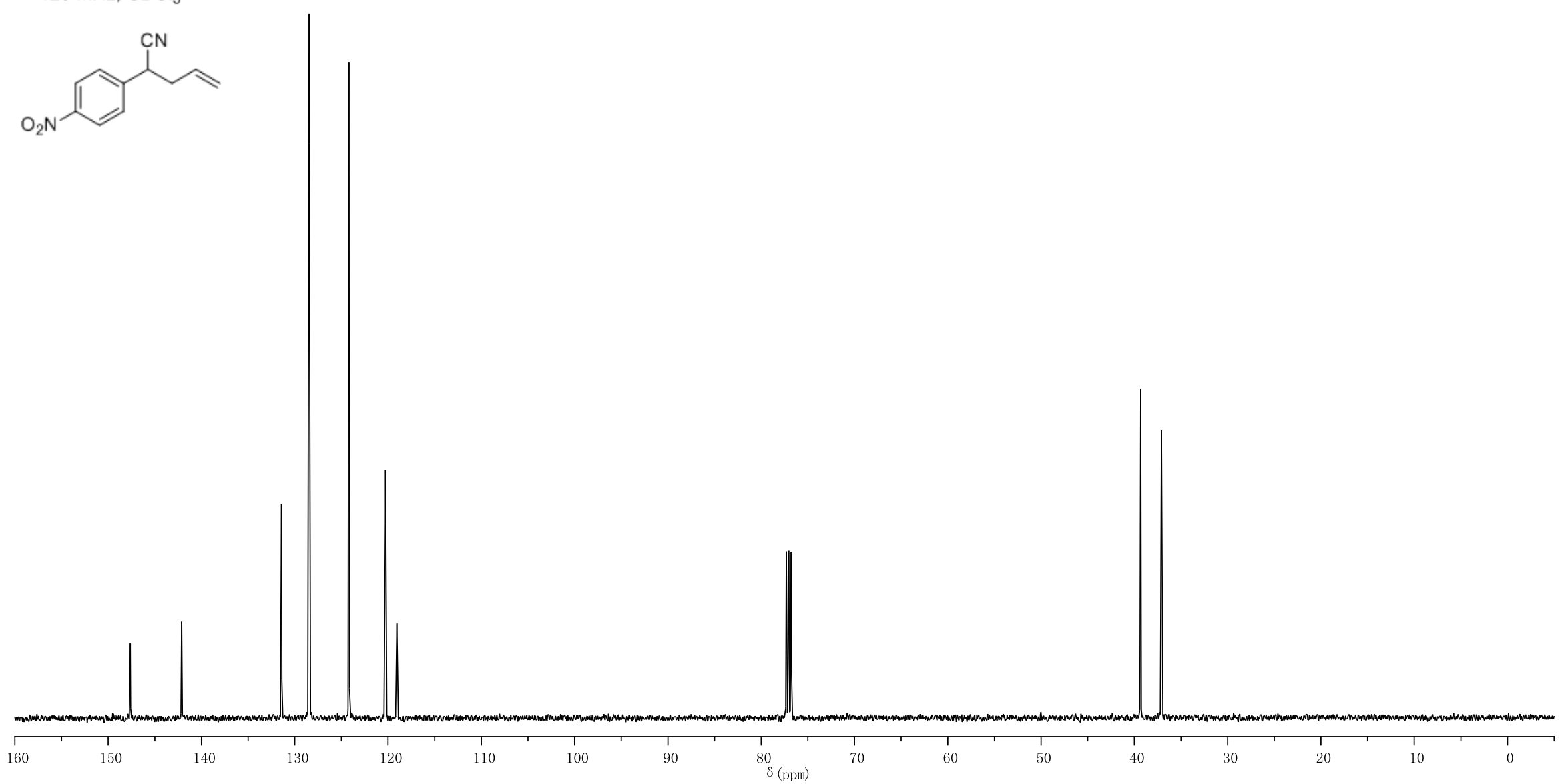




\section{Compound 1zm}

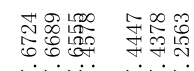

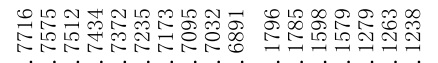

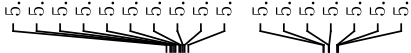

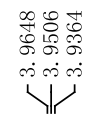

武然

${ }^{1} \mathrm{H}$ NMR

$500 \mathrm{MHz}, \mathrm{CDCl}_{3}$
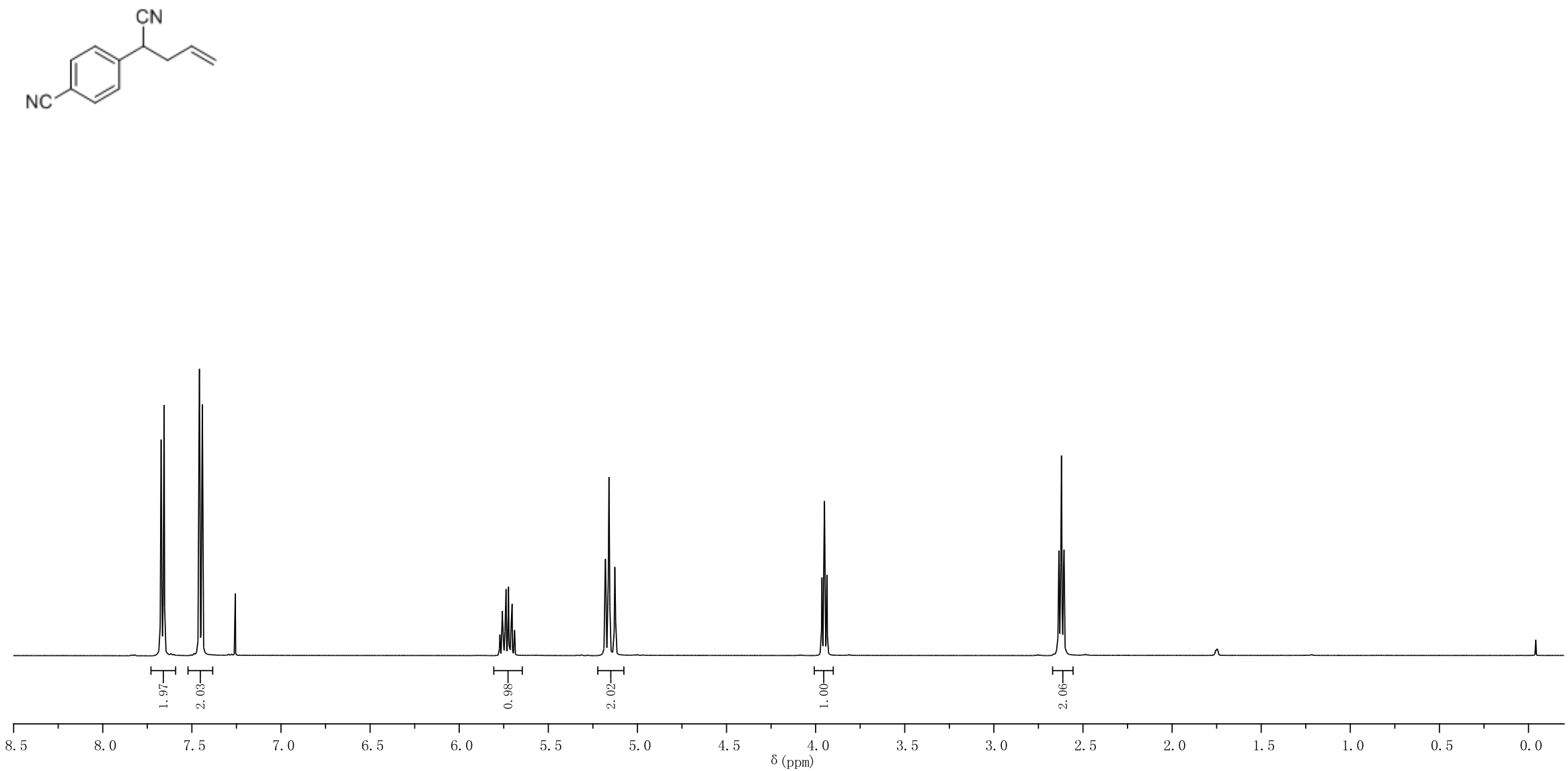
Compound 1zm

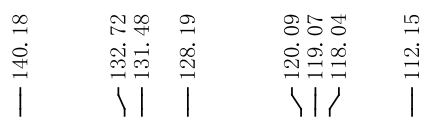

잉용옹

${ }^{13} \mathrm{C}\left\{{ }^{1} \mathrm{H}\right\}$ NMR

$125 \mathrm{MHz}, \mathrm{CDCl}_{3}$
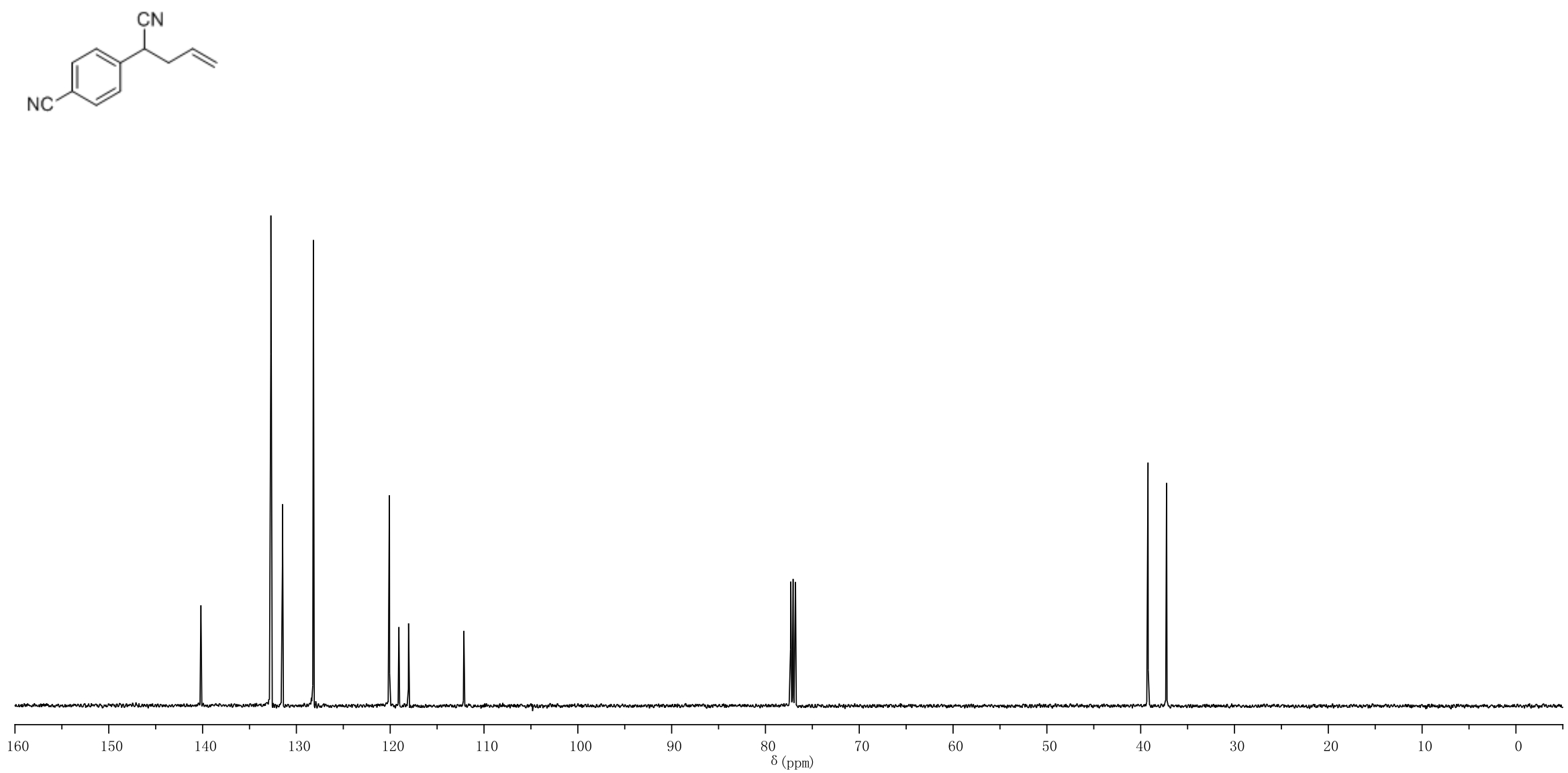
9. Copies of NMR Spectra for $2 t$ and $2 u$

\section{Compound 2t}
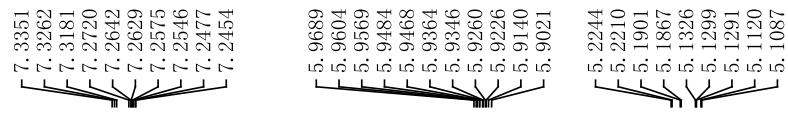

\section{${ }^{1} \mathrm{H}$ NMR}

$500 \mathrm{MHz}, \mathrm{CDCl}_{3}$

$\mathrm{Ph} \widehat{\mathrm{H}} \widehat{\mathrm{N}}$

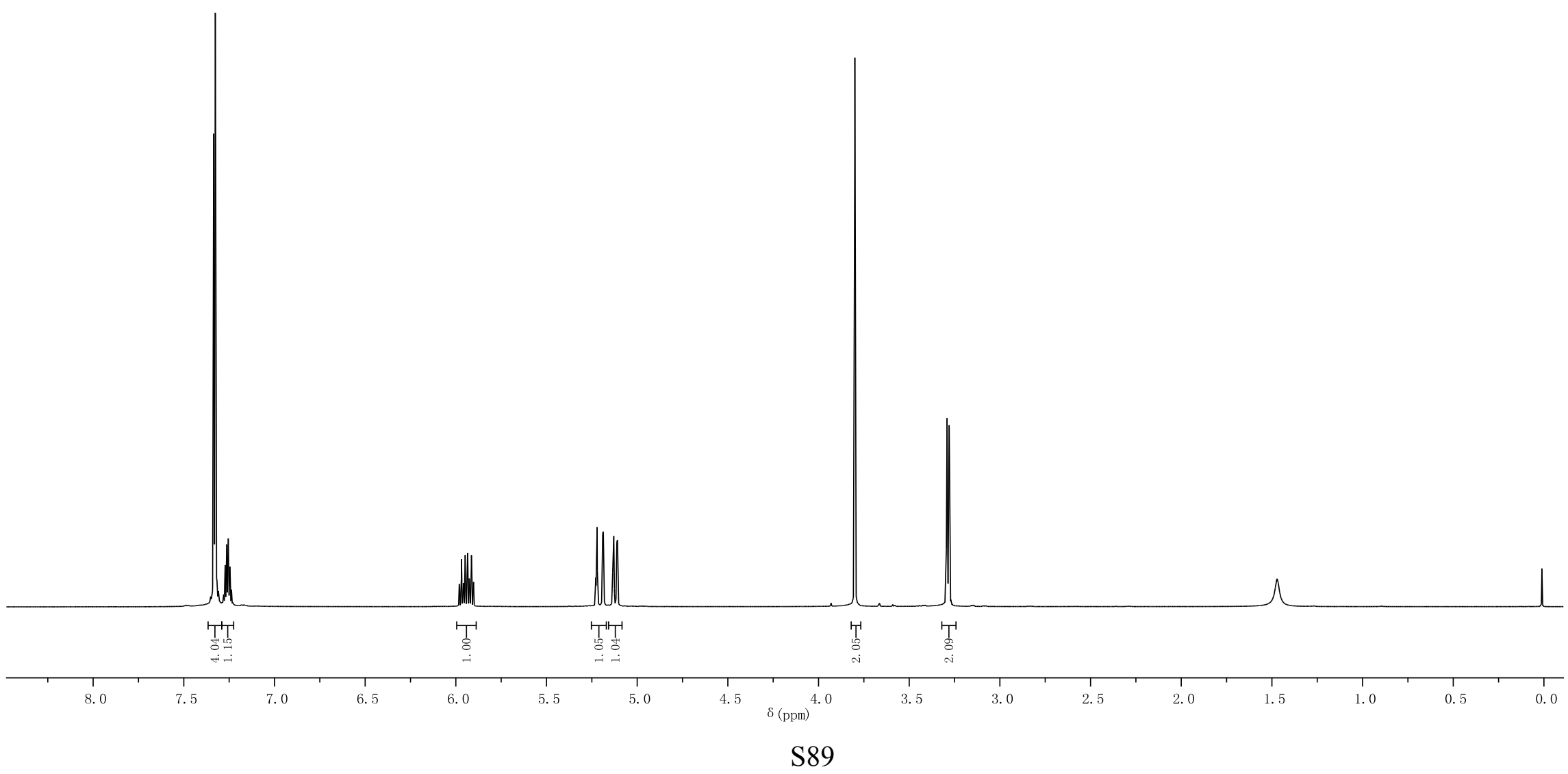




\section{Compound 2t}

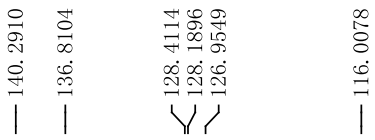

${ }^{13} \mathrm{C}\left\{{ }^{1} \mathrm{H}\right\}$ NMR

$125 \mathrm{MHz}, \mathrm{CDCl}_{3}$

$\mathrm{Ph} \widehat{\mathrm{H}} \widehat{\mathrm{N}}$

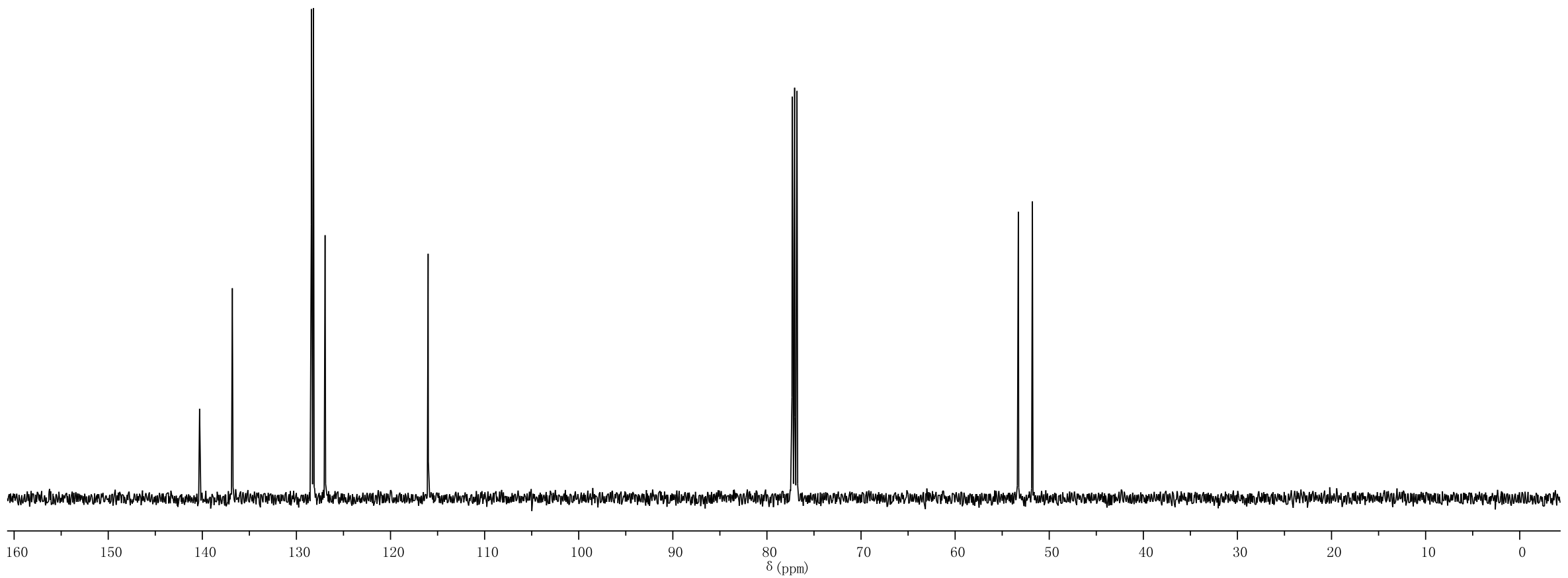




\section{Compound 2u}

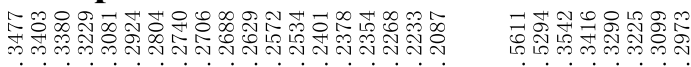

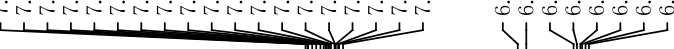

${ }^{1} \mathrm{H}$ NMR

$500 \mathrm{MHz}, \mathrm{CDCl}_{3}$

$\mathrm{Ph} \widehat{\mathrm{H}} \widehat{\sim} \widehat{P h}_{\mathrm{Ph}}$

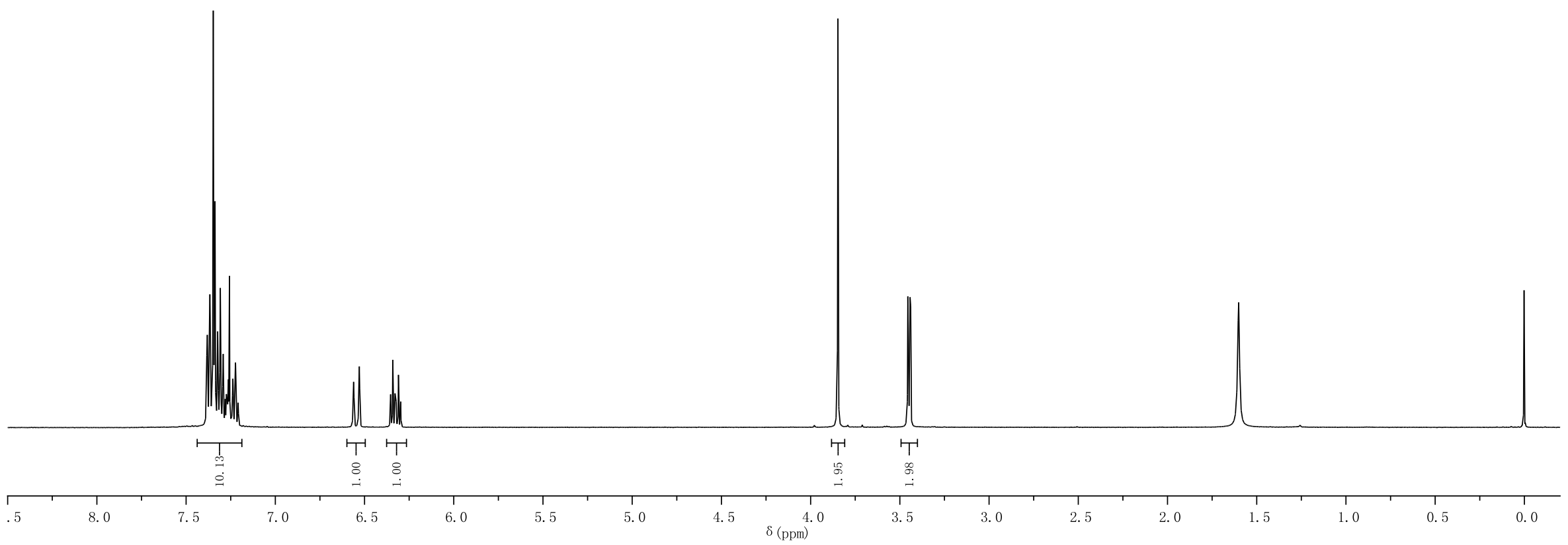


Compound 2u

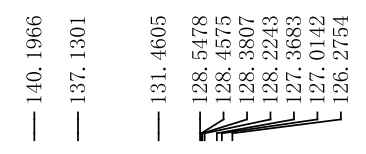

${ }^{13} \mathrm{C}\left\{{ }^{1} \mathrm{H}\right\}$ NMR

$125 \mathrm{MHz}, \mathrm{CDCl}_{3}$

$\mathrm{Ph} \widehat{\mathrm{H}} \widehat{\sim} \widehat{P h}_{\mathrm{Ph}}$

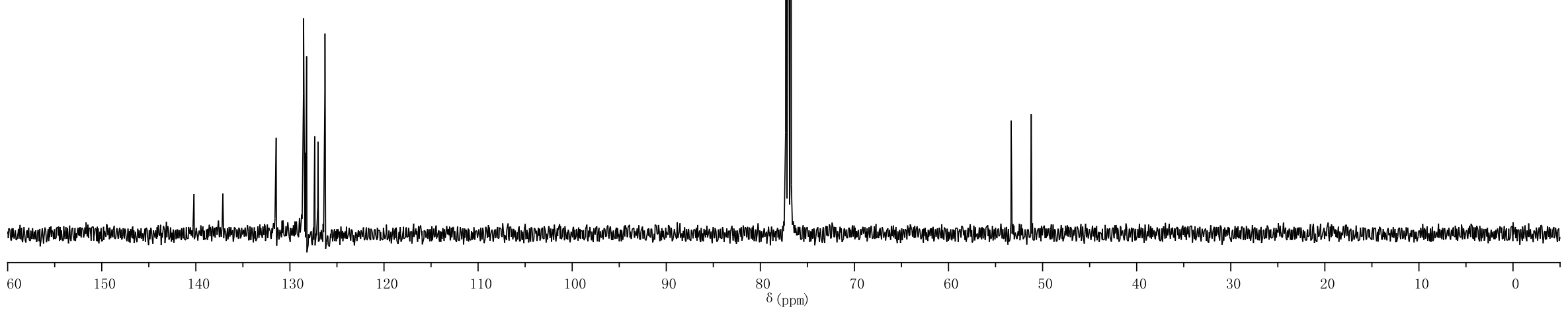


10. Copies of NMR spectra for 3

\section{Compound ( \pm )-3aa}

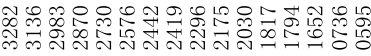

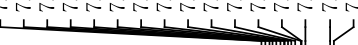

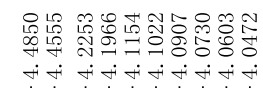

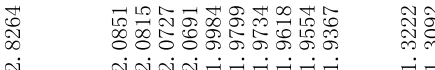

$\sqrt{11}$

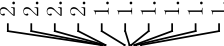

wiv

${ }^{1} \mathrm{H}$ NMR

$500 \mathrm{MHz}, \mathrm{CDCl}_{3}$

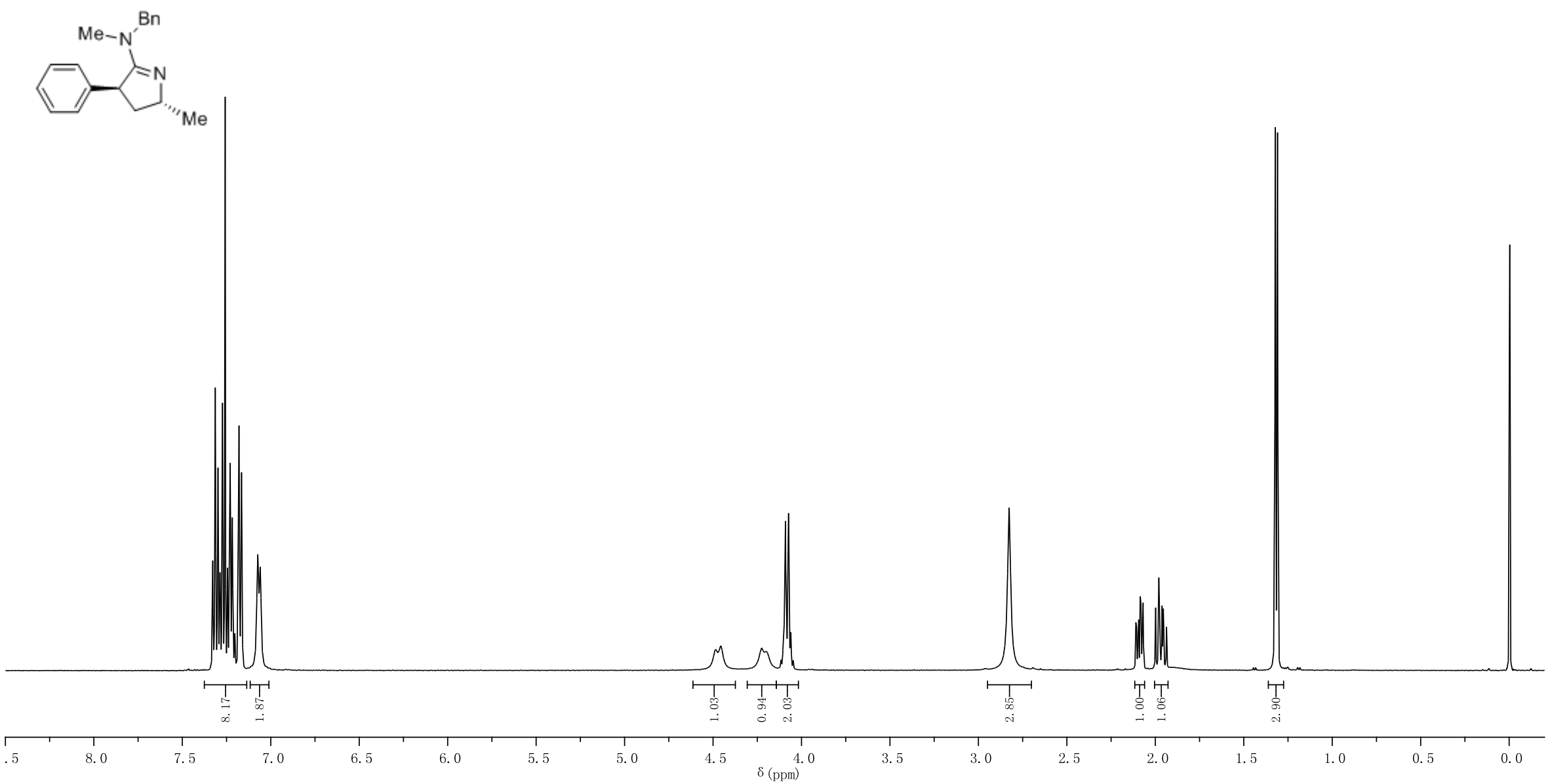




\section{Compound ( \pm )-3aa}

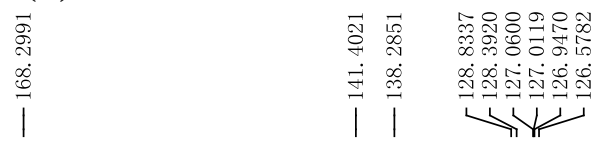

${ }^{13} \mathrm{C}\left\{{ }^{1} \mathrm{H}\right\} \mathrm{NMR}$

$125 \mathrm{MHz}, \mathrm{CDCl}_{3}$
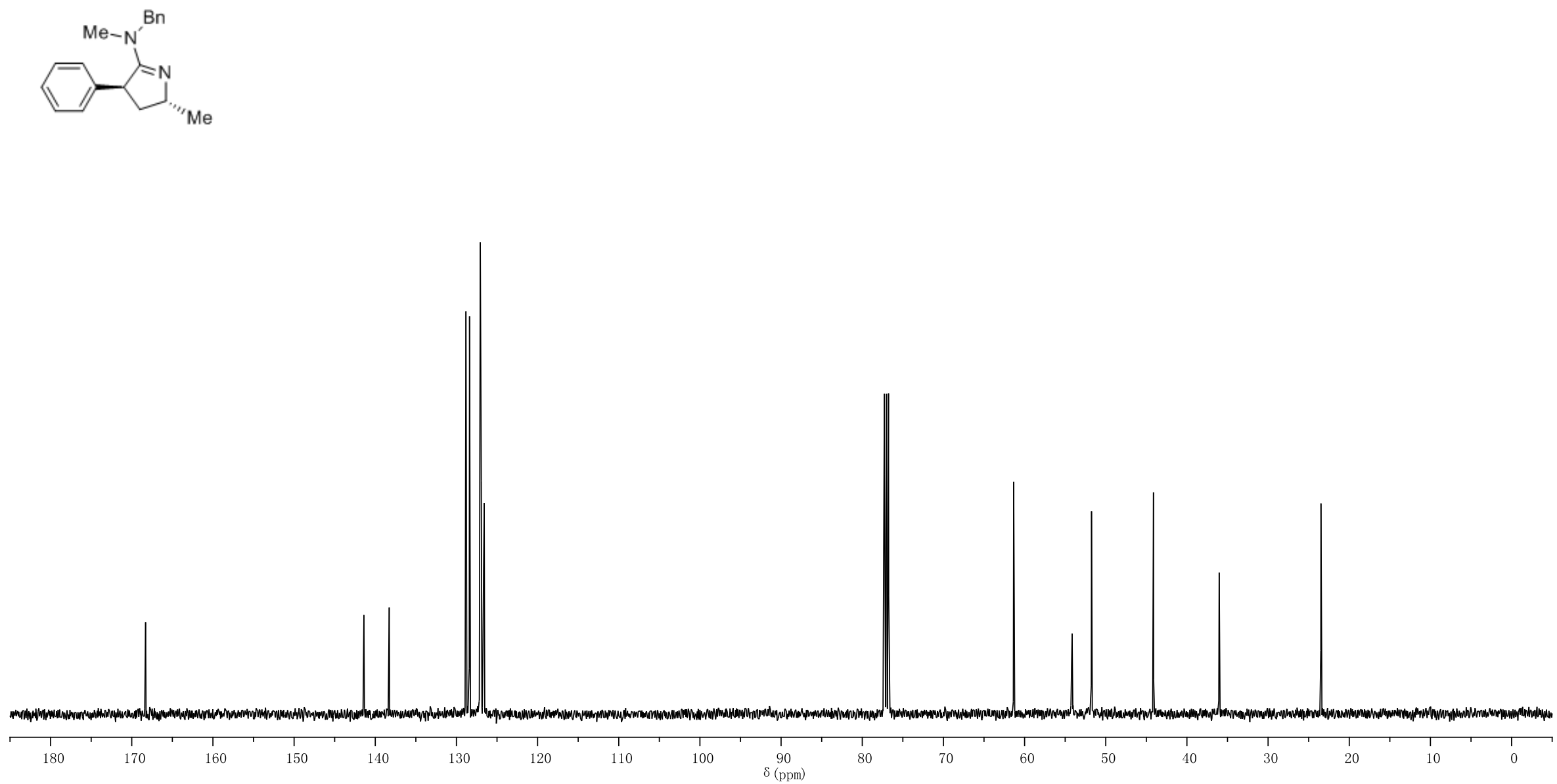


\section{Compound ( \pm )-3ba}

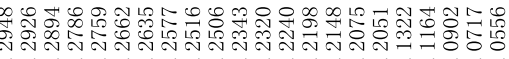

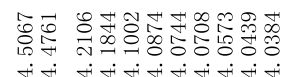

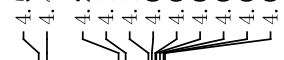

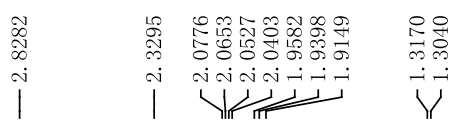

${ }^{1} \mathrm{H}$ NMR

$500 \mathrm{MHz}, \mathrm{CDCl}_{3}$
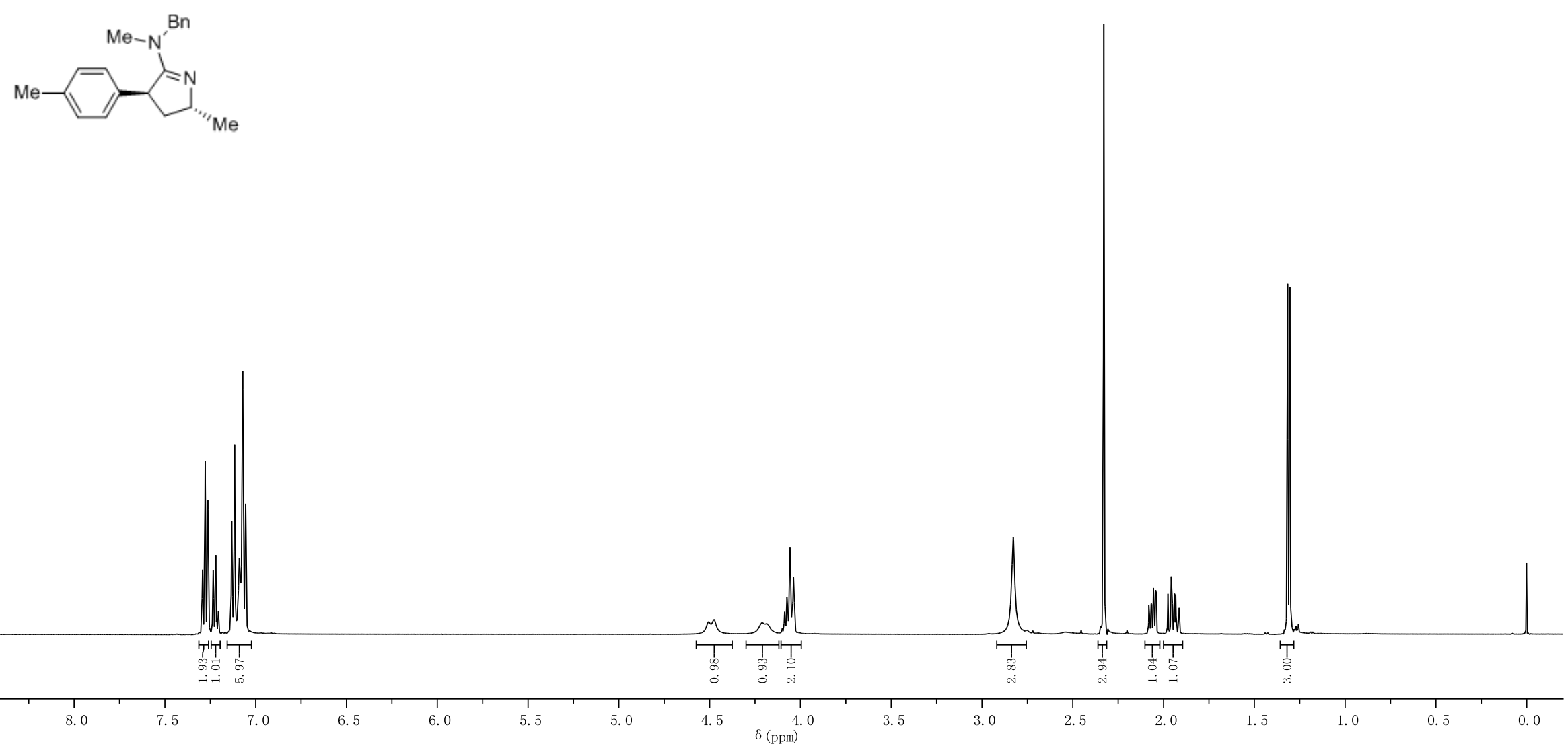


\section{Compound ( \pm )-3ca}

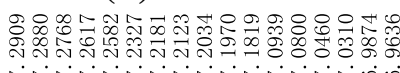

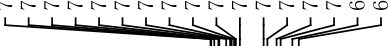

${ }^{1} \mathrm{H}$ NMR

$500 \mathrm{MHz}, \mathrm{CDCl}_{3}$
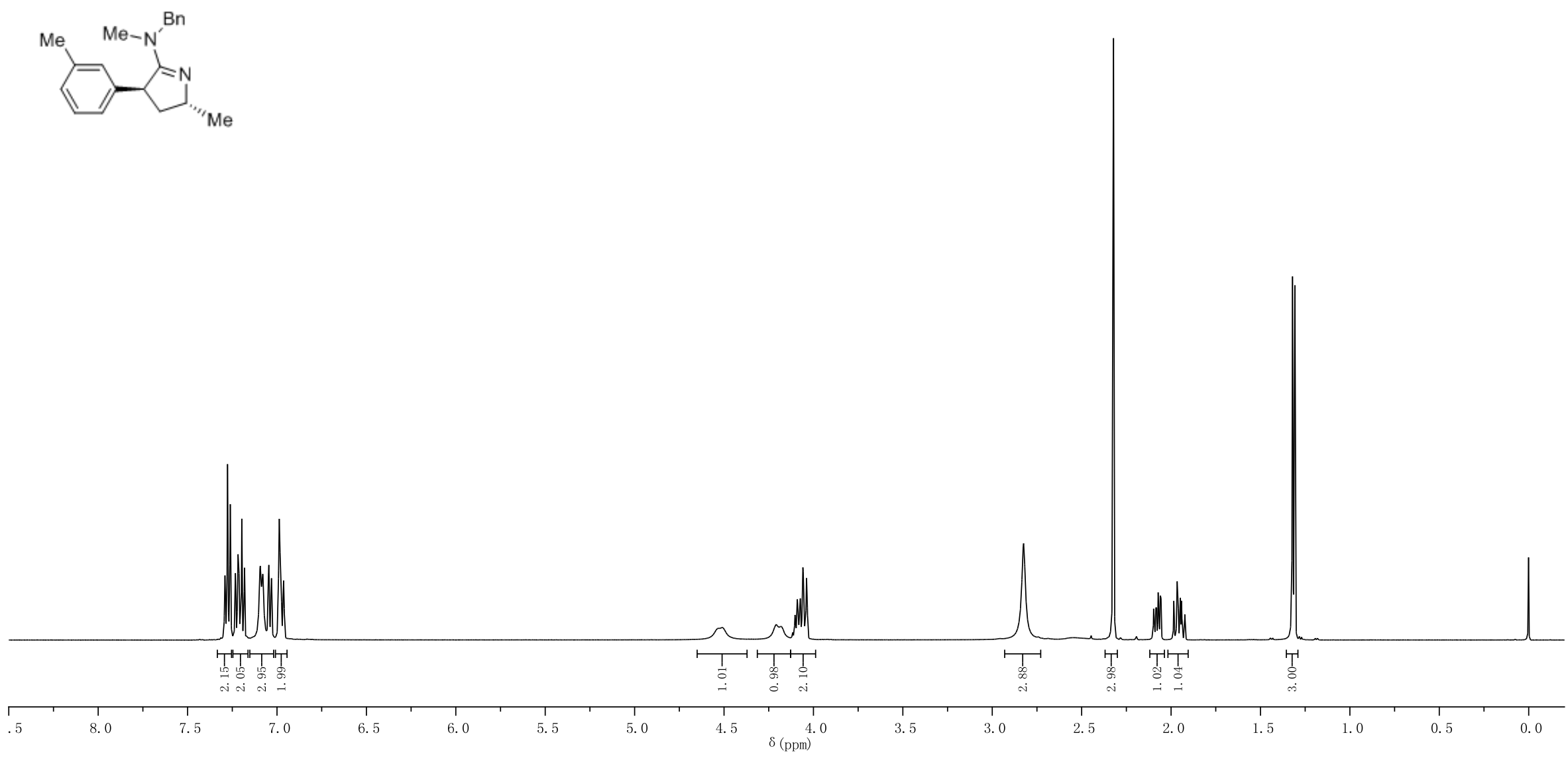


\section{Compound ( \pm )-3ca}

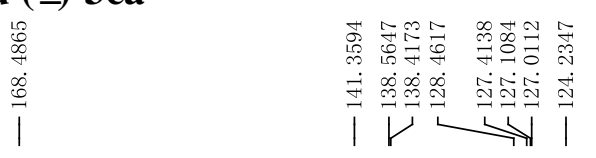

${ }^{13} \mathrm{C}\left\{{ }^{1} \mathrm{H}\right\}$ NMR

$125 \mathrm{MHz}, \mathrm{CDCl}_{3}$
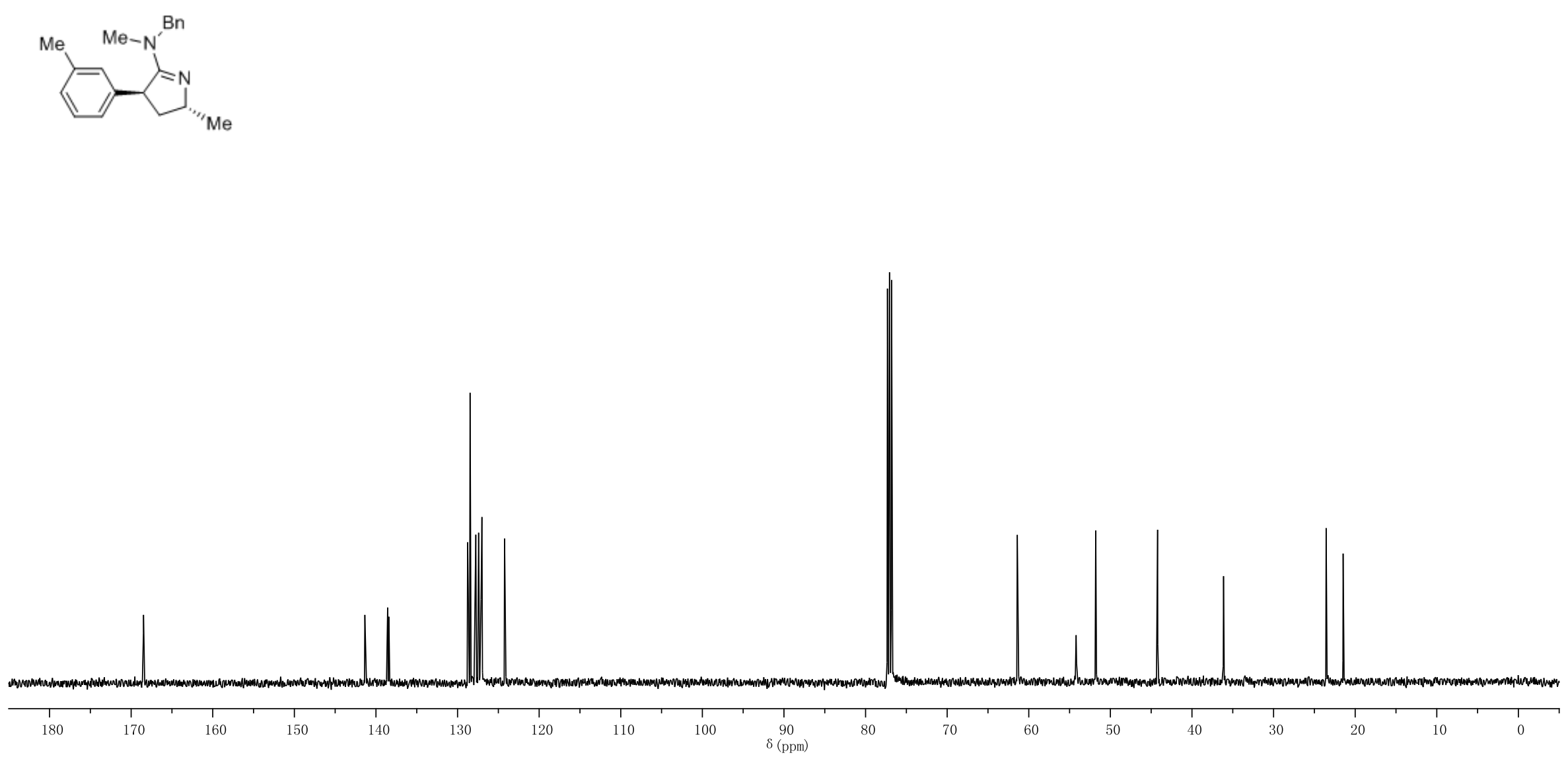


\section{Compound ( \pm )-3da}

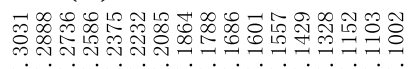

in

mImInIII

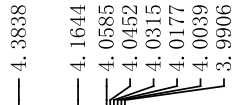

${ }^{1} \mathrm{H}$ NMR

$500 \mathrm{MHz}, \mathrm{CDCl}_{3}$
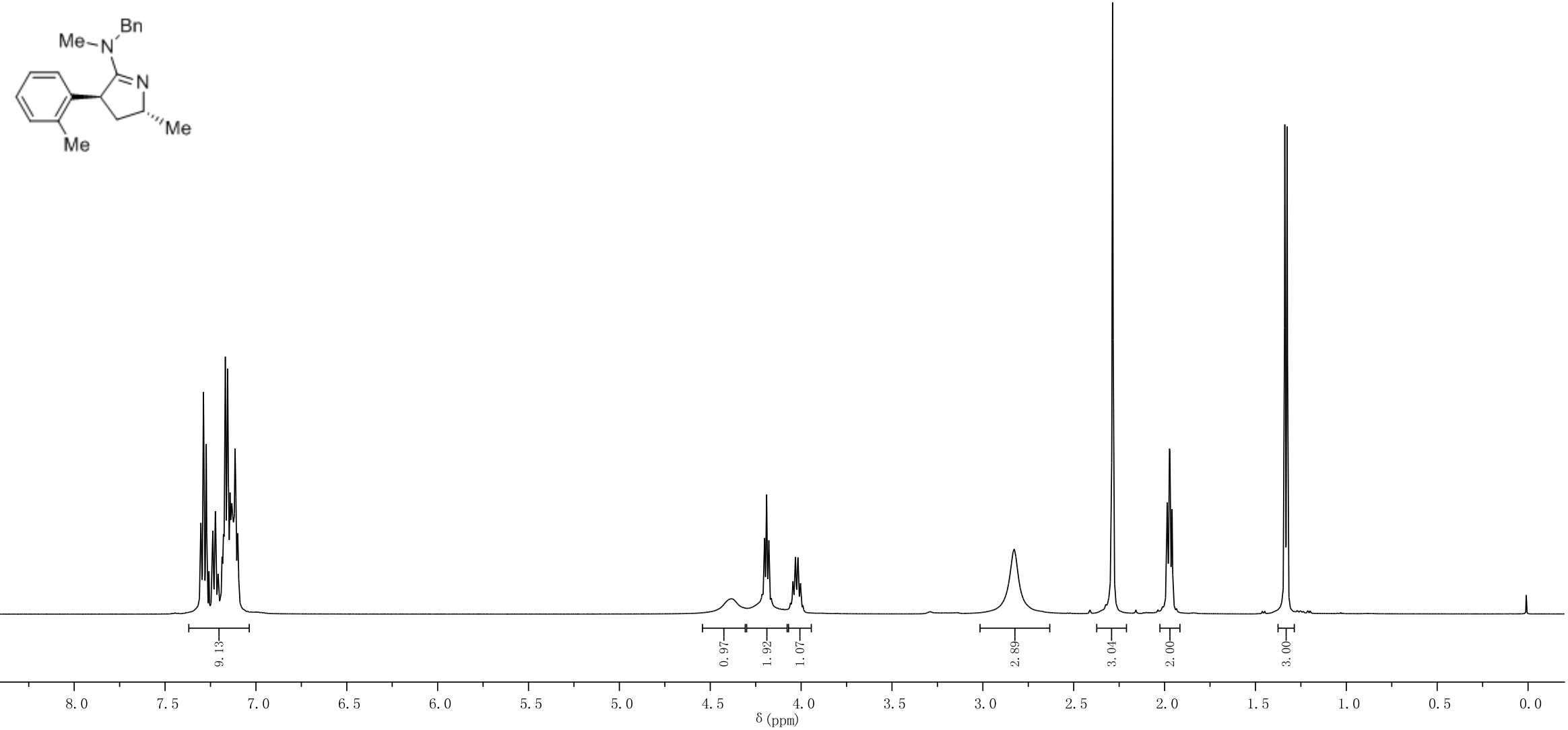


\section{Compound ( \pm )-3da}

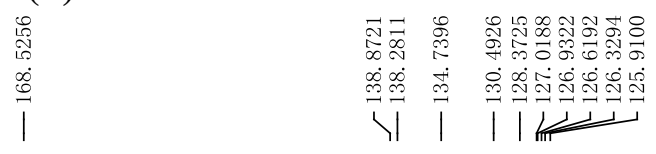

${ }^{13} \mathrm{C}\left\{{ }^{1} \mathrm{H}\right\}$ NMR

$125 \mathrm{MHz}, \mathrm{CDCl}_{3}$
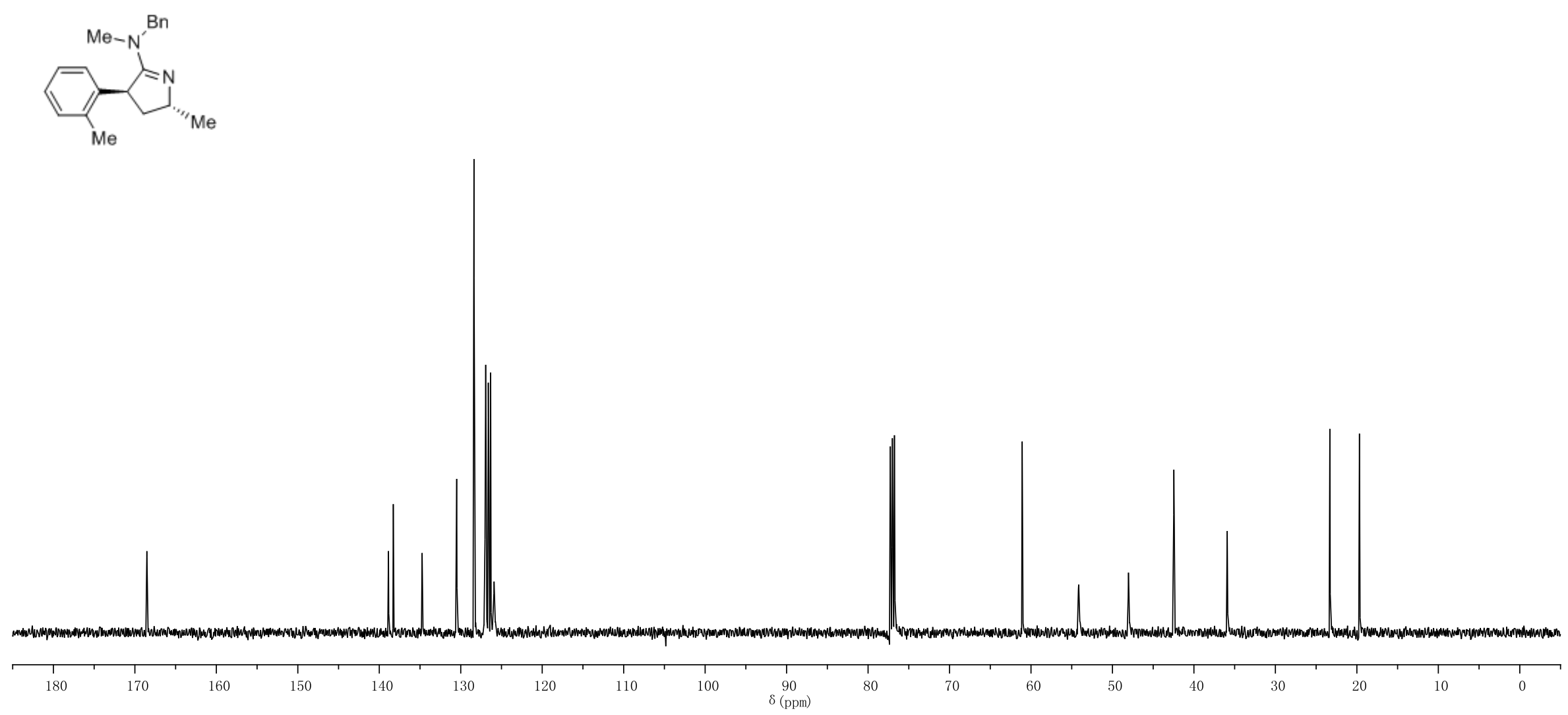


\section{Compound ( \pm )-3ea}

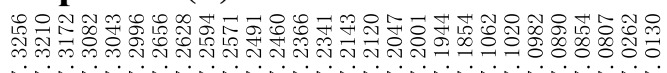

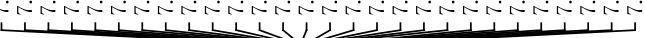

${ }^{1} \mathrm{H}$ NMR

$500 \mathrm{MHz} \mathrm{CDCl}_{3}$

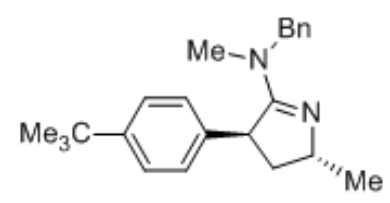

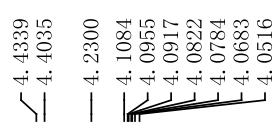

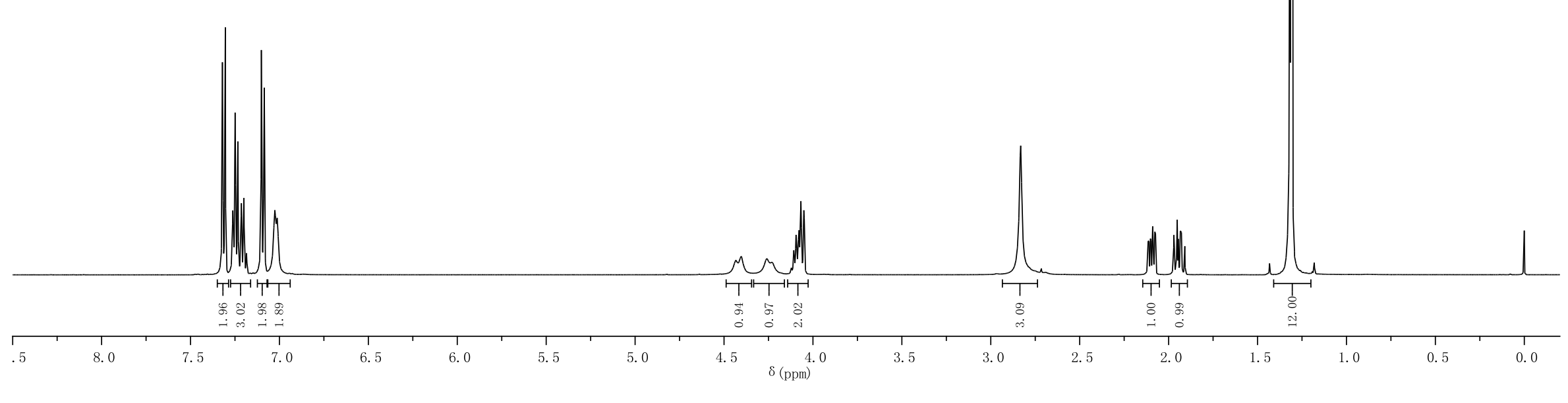




\section{Compound ( \pm )-3ea}

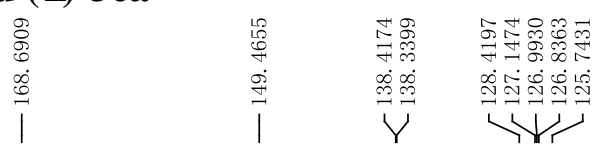

${ }^{13} \mathrm{C}\left\{{ }^{1} \mathrm{H}\right\}$ NMR

$125 \mathrm{MHz}, \mathrm{CDCl}_{3}$
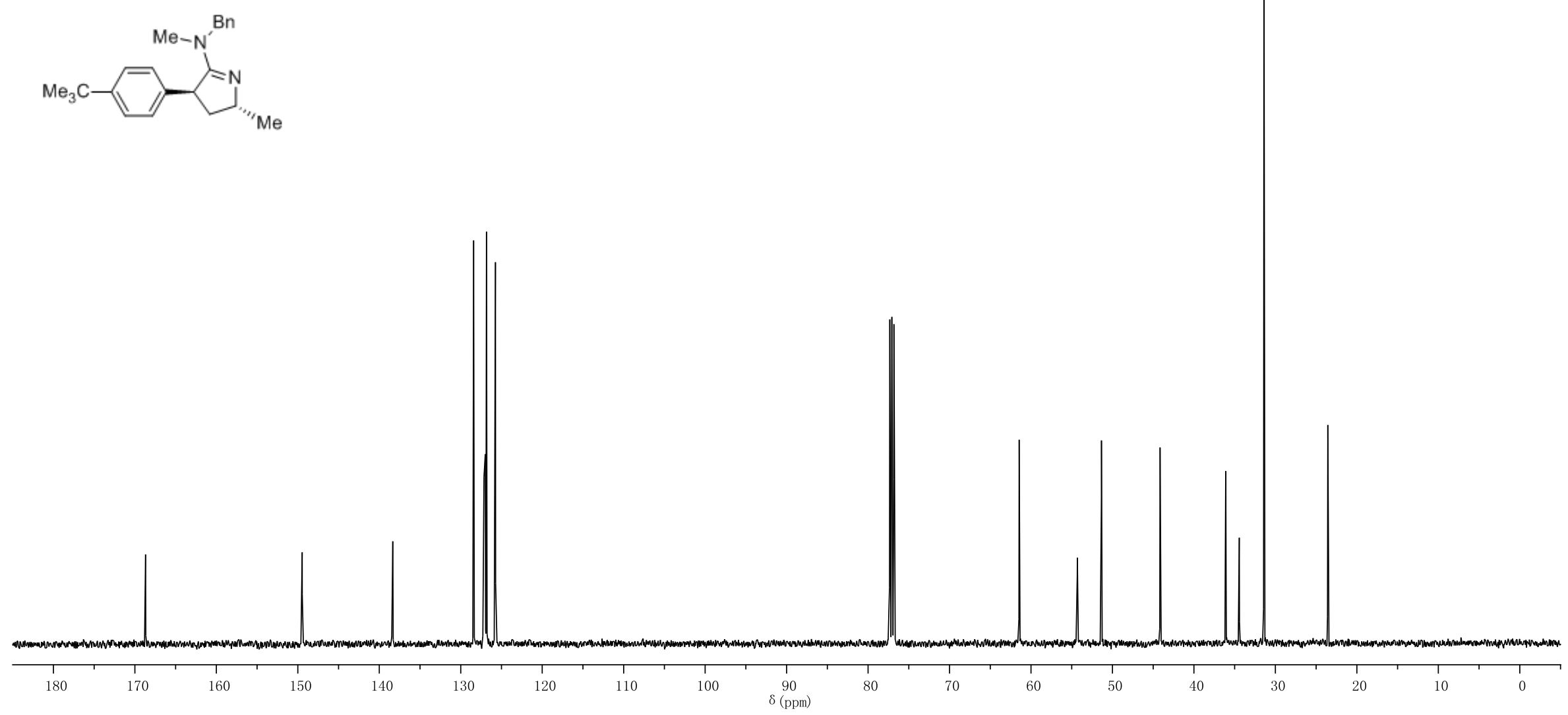


\section{Compound ( \pm )-3fa}

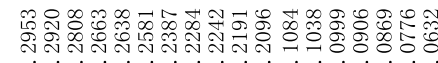

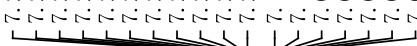

${ }^{1} \mathrm{H}$ NMR

$500 \mathrm{MHz}, \mathrm{CDCl}_{3}$
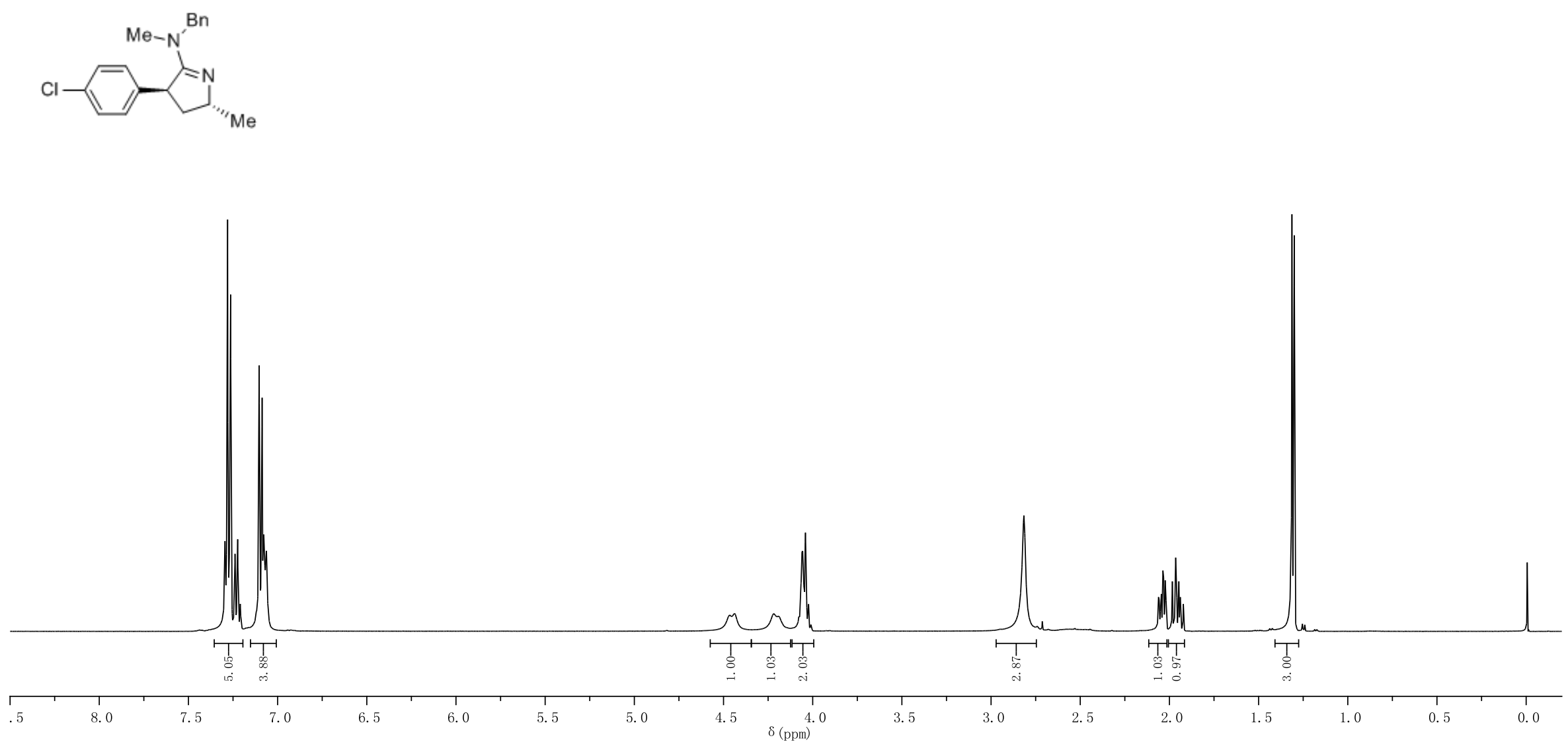

S103

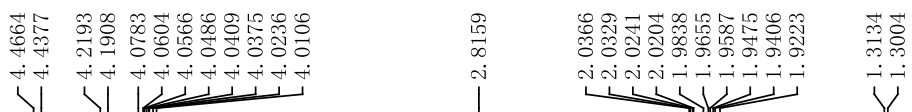

${ }^{2}$ 
Compound ( \pm )-3fa

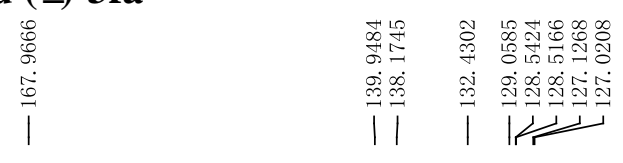

${ }^{13} \mathrm{C}\left\{{ }^{1} \mathrm{H}\right\} \mathrm{NMR}$

$125 \mathrm{MHz}, \mathrm{CDCl}_{3}$

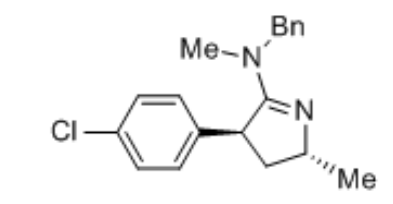

$\mathrm{Me}$

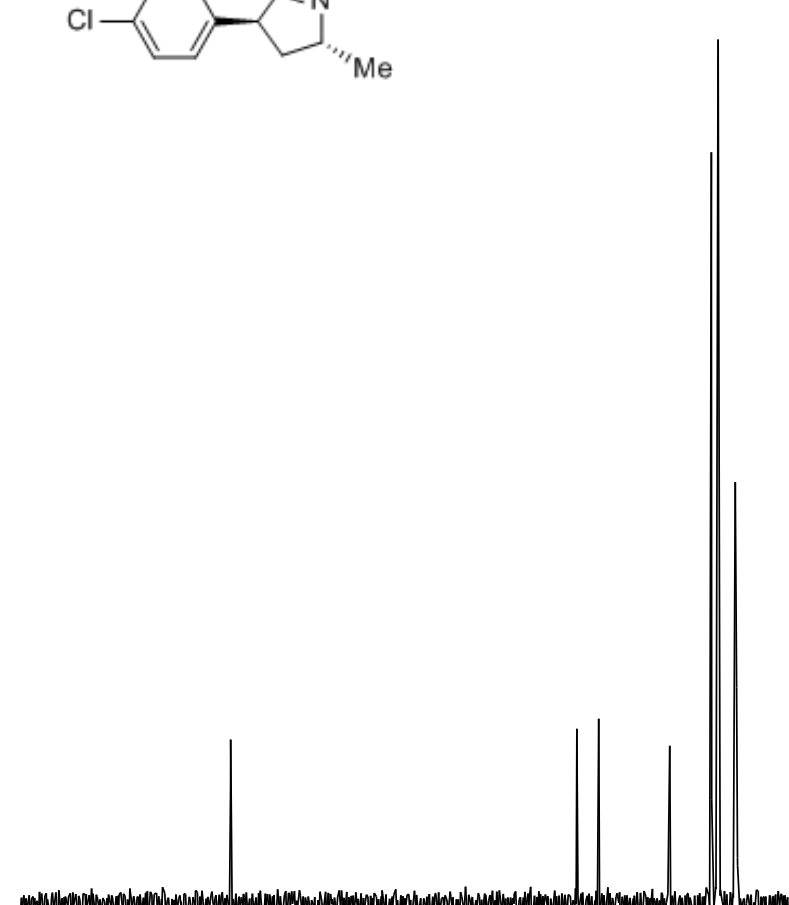

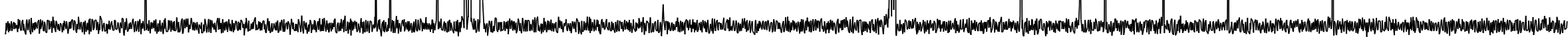




\section{Compound ( \pm )-3ga}

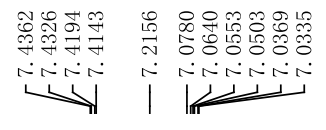

${ }^{1} \mathrm{H}$ NMR

$500 \mathrm{MHz}, \mathrm{CDCl}_{3}$
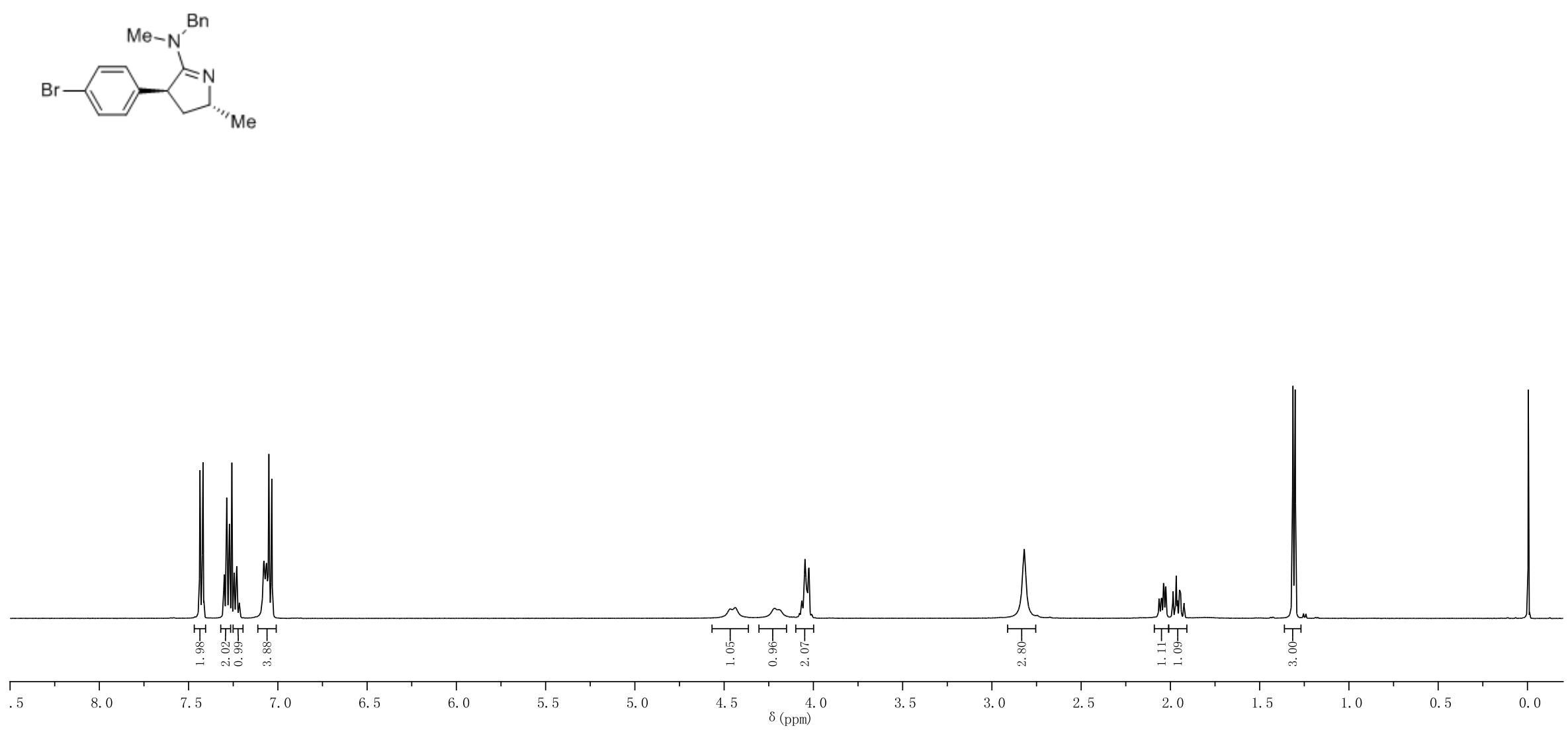
Compound ( \pm )-3ga

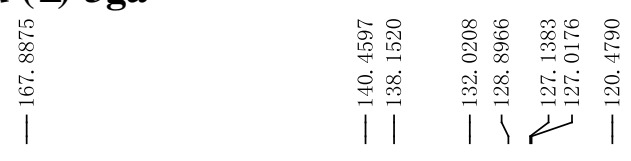

${ }^{13} \mathrm{C}\left\{{ }^{1} \mathrm{H}\right\} \mathrm{NMR}$

$125 \mathrm{MHz} \mathrm{CDCl}_{3}$

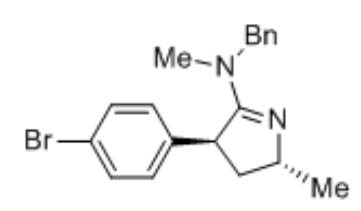

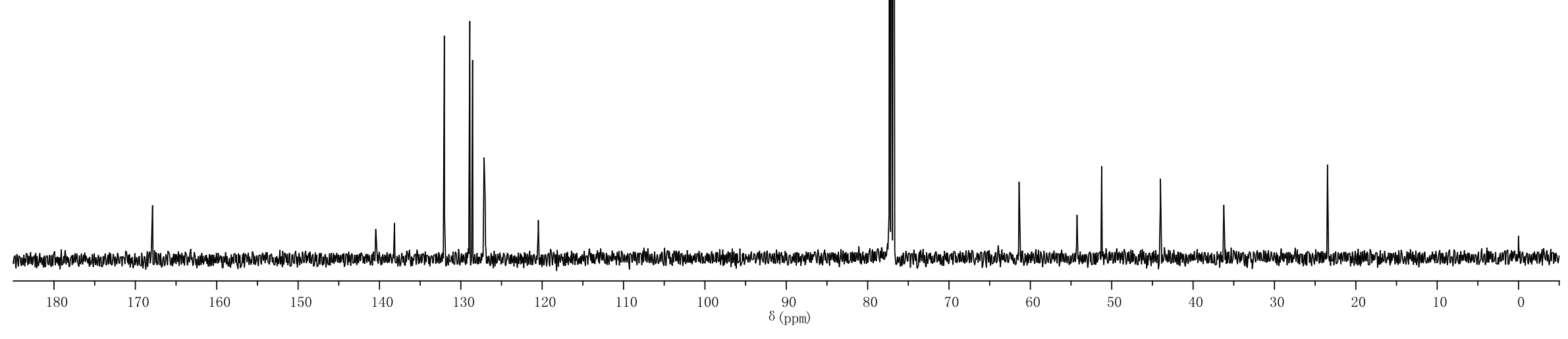




\section{Compound ( \pm )-3ha}

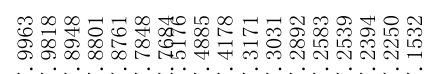

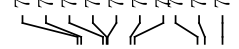

${ }^{1} \mathrm{H}$ NMR

$500 \mathrm{MHz}, \mathrm{CDCl}_{3}$
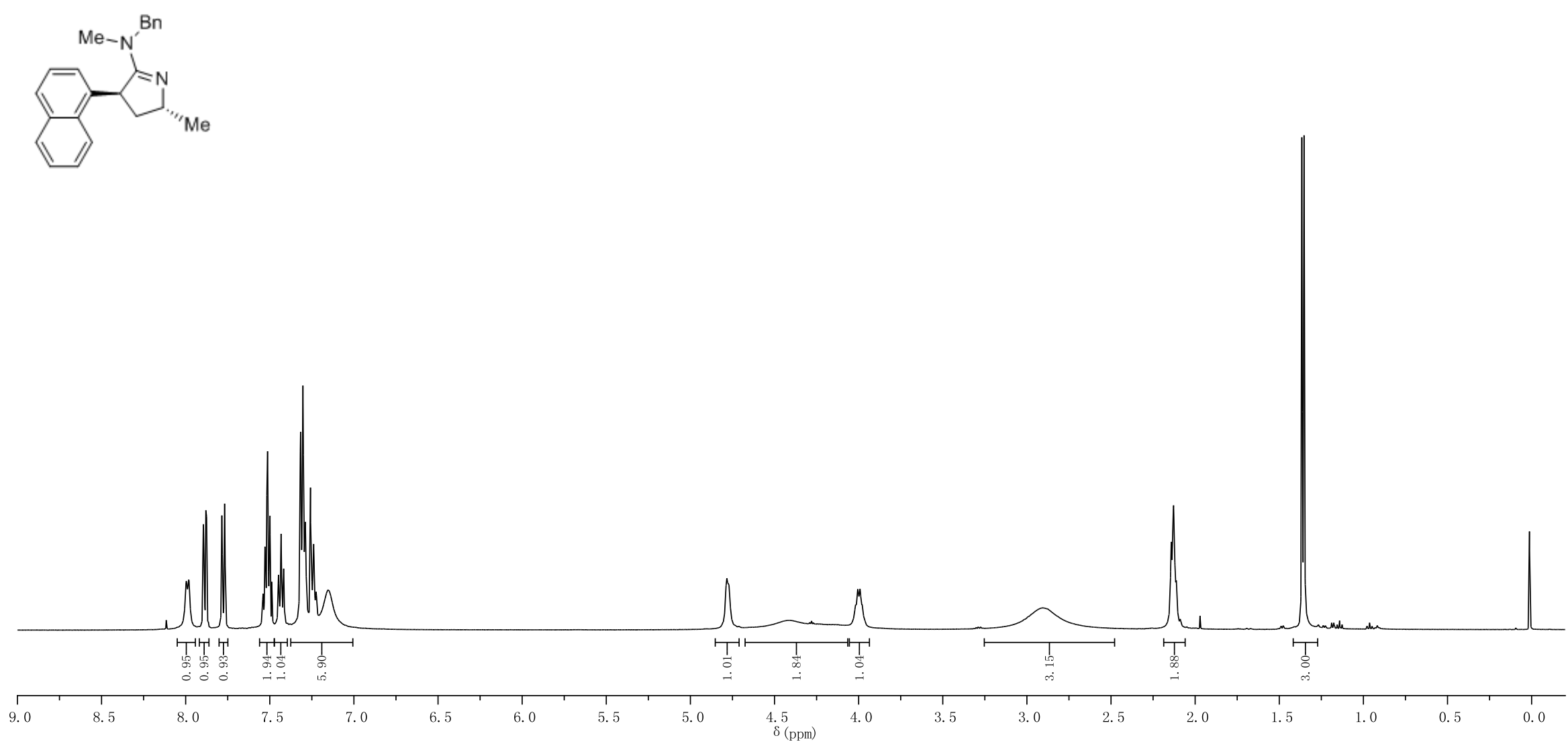
Compound ( \pm )-3ha

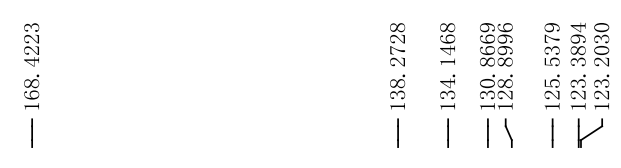

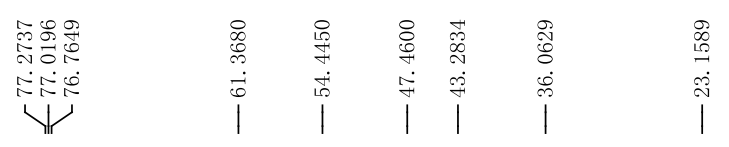

${ }^{13} \mathrm{C}\left\{{ }^{1} \mathrm{H}\right\}$ NMR

$125 \mathrm{MHz}, \mathrm{CDCl}_{3}$
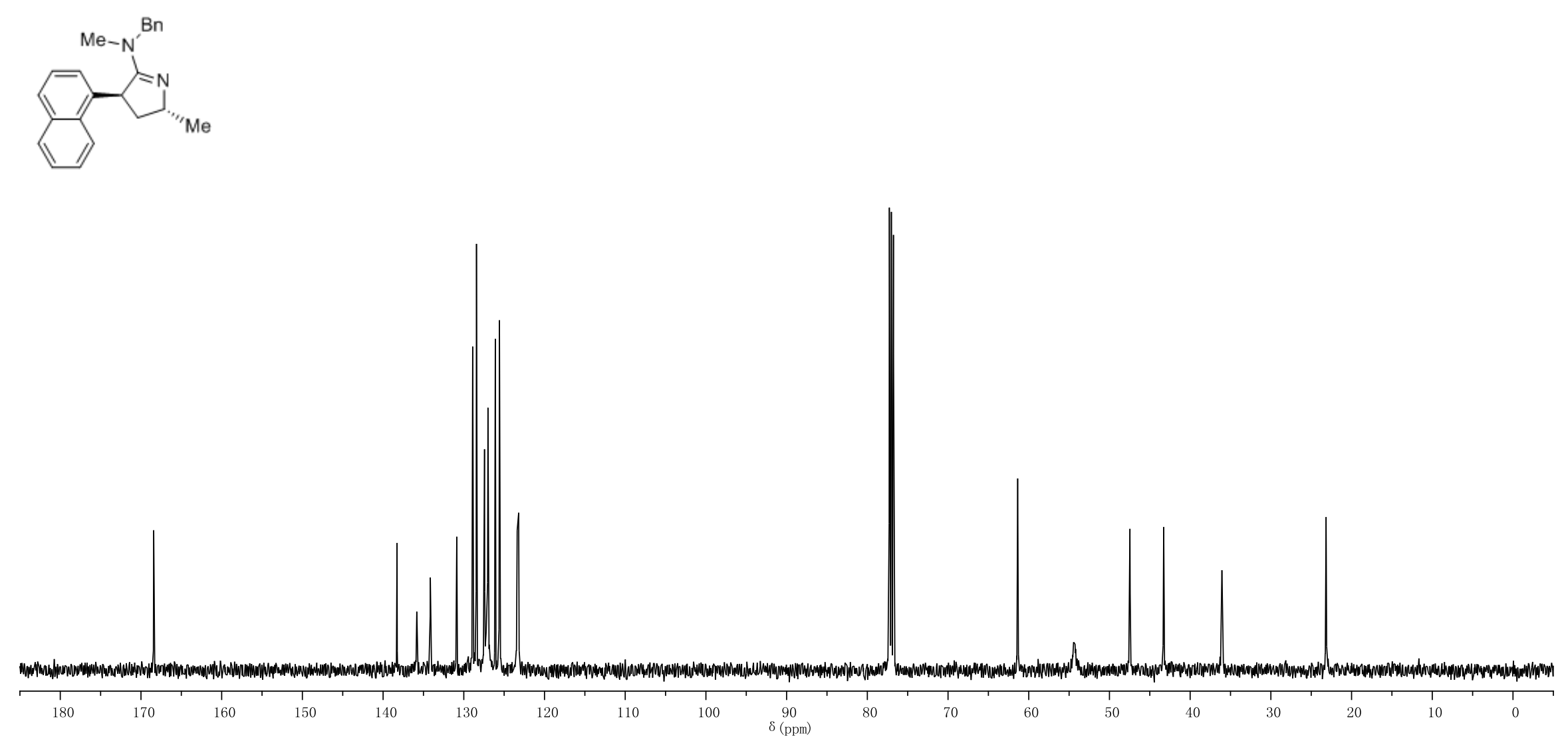


\section{Compound ( \pm )-3ia}

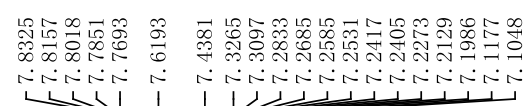

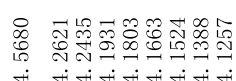

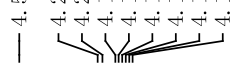

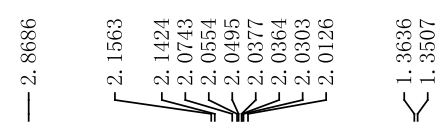

${ }^{1} \mathrm{H}$ NMR

$500 \mathrm{MHz}, \mathrm{CDCl}_{3}$
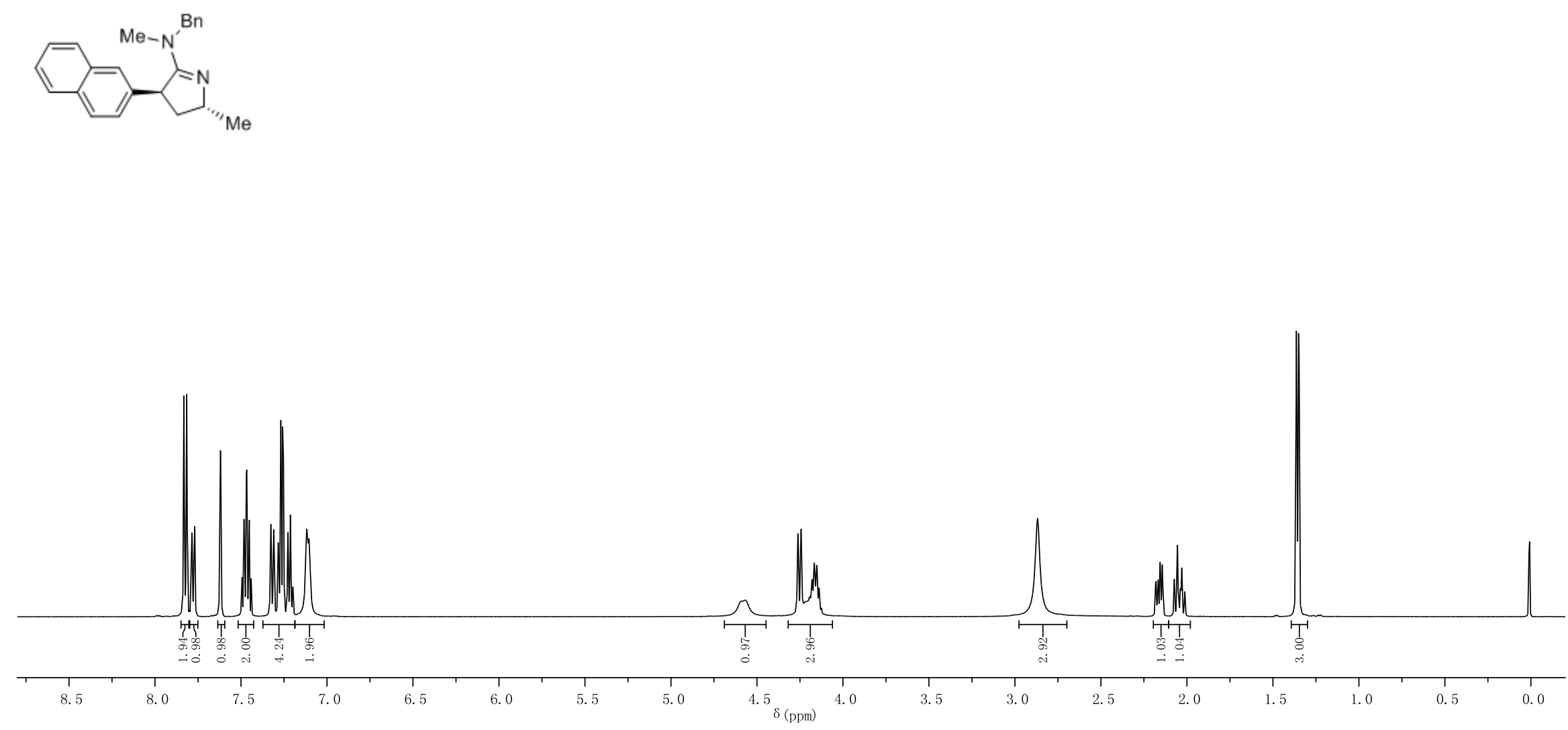

S109 


\section{Compound ( \pm )-3ia}

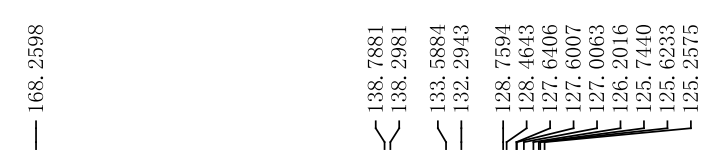

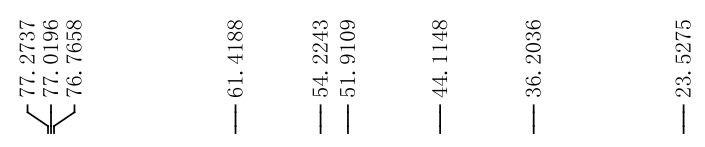

${ }^{13} \mathrm{C}\left\{{ }^{1} \mathrm{H}\right\}$ NMR

$125 \mathrm{MHz}, \mathrm{CDCl}_{3}$
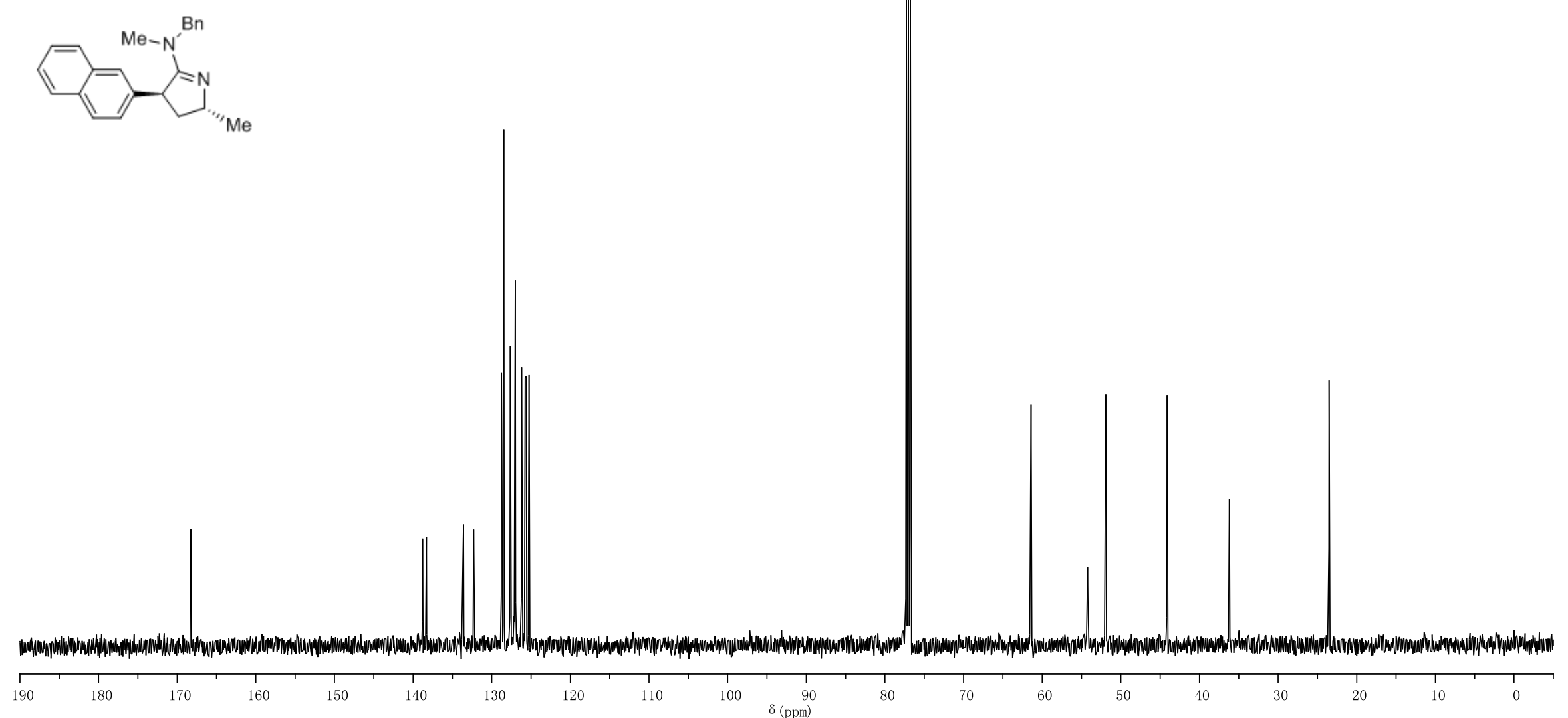


\section{Compound ( \pm$)-3 \mathbf{j a}$}

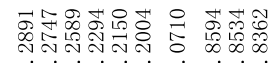

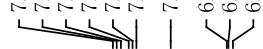

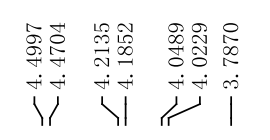

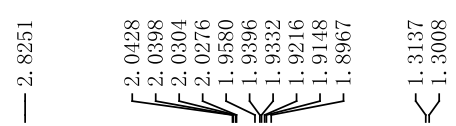

${ }^{1} \mathrm{H}$ NMR

$500 \mathrm{MHz}, \mathrm{CDCl}_{3}$
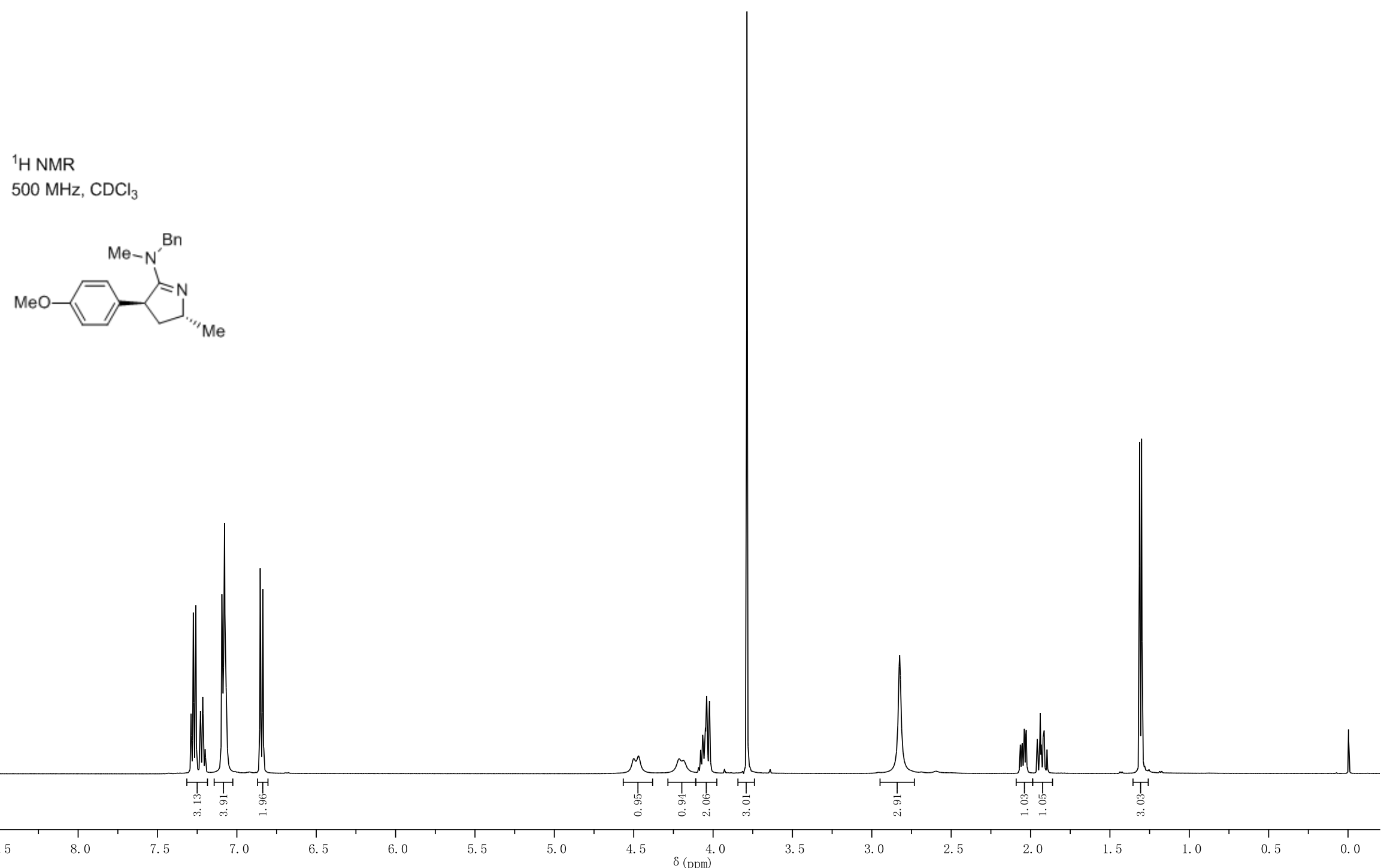


\section{Compound ( \pm )-3ja}

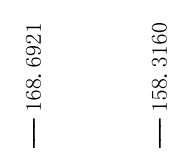

${ }^{13} \mathrm{C}\left\{{ }^{1} \mathrm{H}\right\}$ NMR

$125 \mathrm{MHz}, \mathrm{CDCl}_{3}$
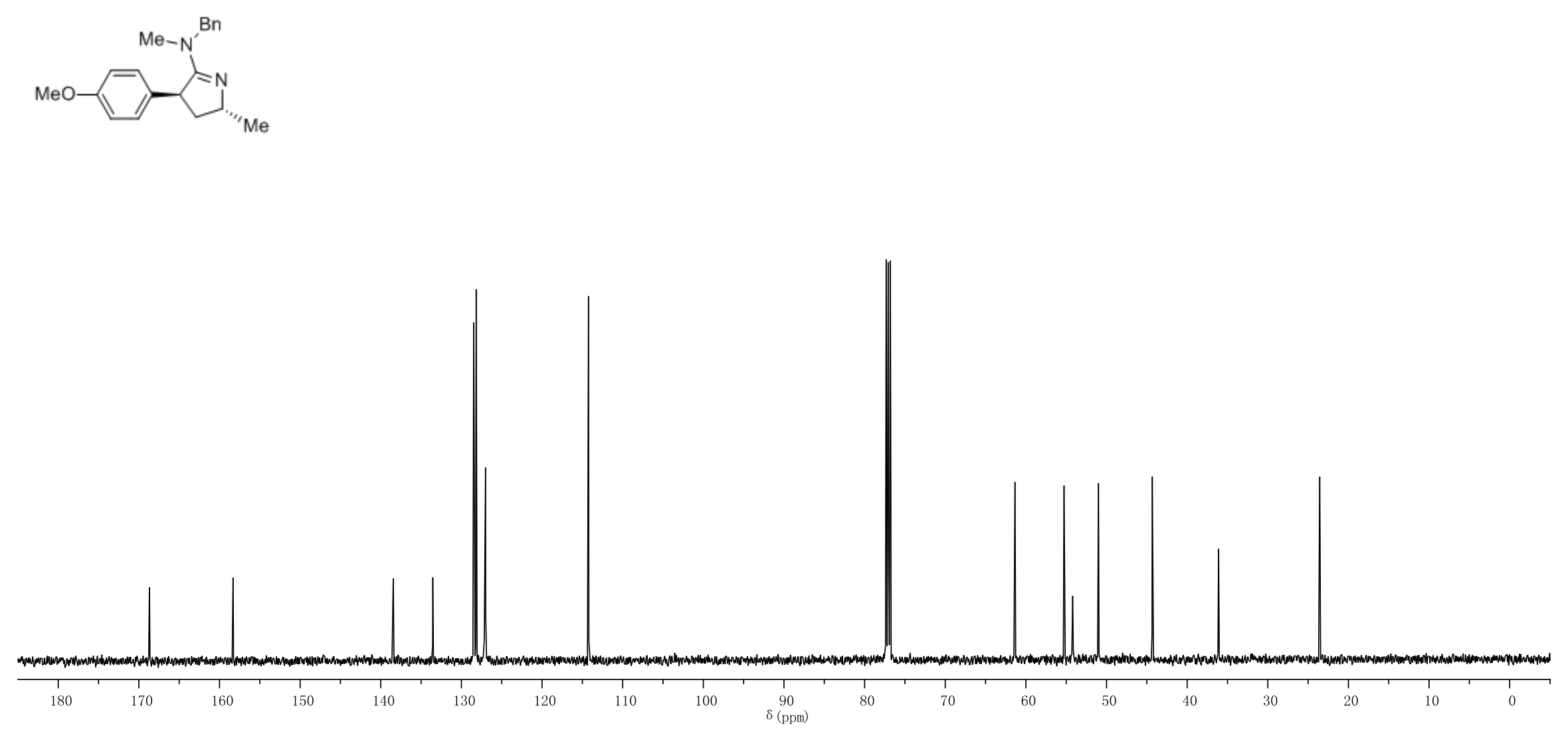


\section{Compound ( \pm )-3ka}

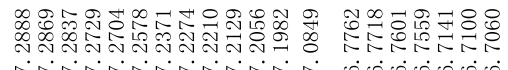

ن

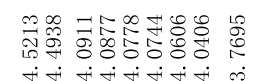

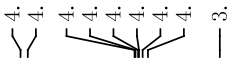

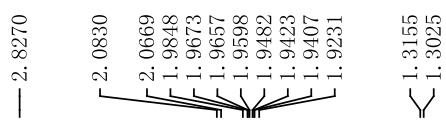

${ }^{1} \mathrm{H}$ NMR

$500 \mathrm{MHz}, \mathrm{CDCl}_{3}$

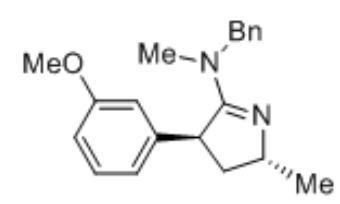

"Me

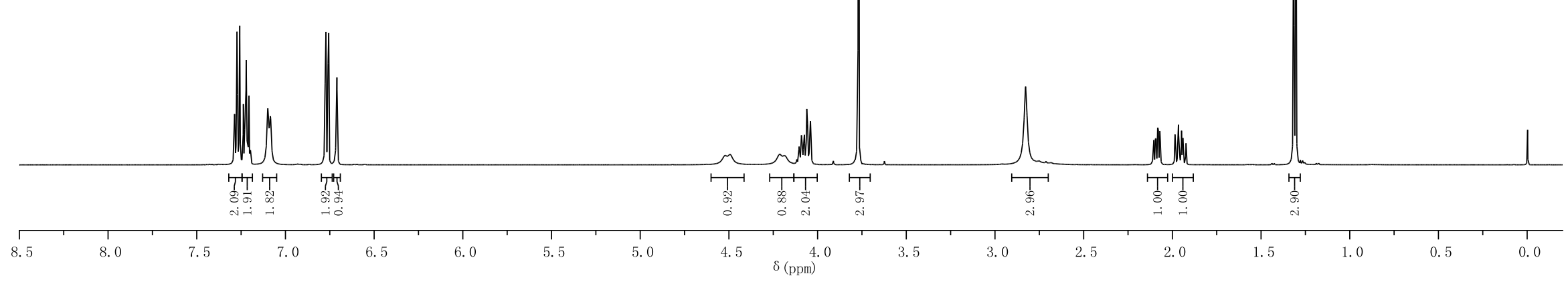


Compound ( \pm )-3ka

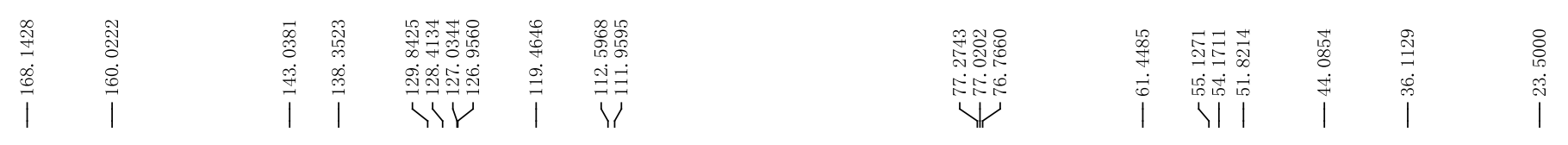

${ }^{13} \mathrm{C}\left\{{ }^{1} \mathrm{H}\right\}$ NMR

$125 \mathrm{MHz}, \mathrm{CDCl}_{3}$
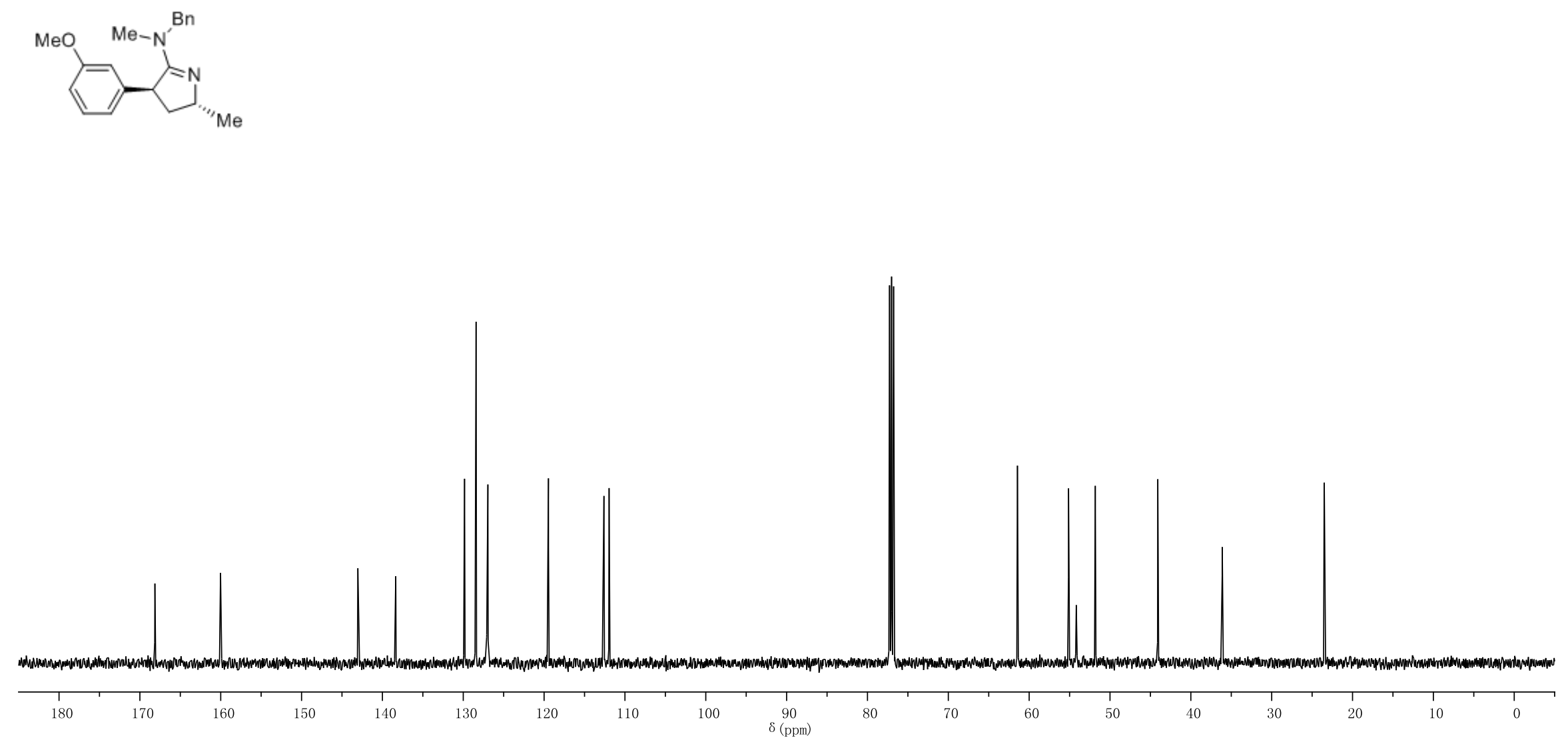


\section{Compound ( \pm )-3la}

范

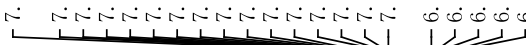

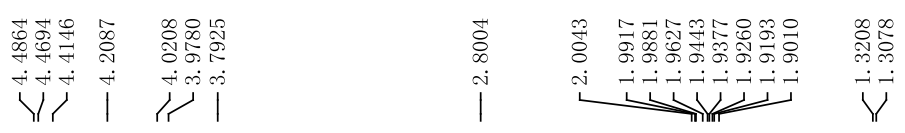

${ }^{1} \mathrm{H}$ NMR

$500 \mathrm{MHz}, \mathrm{CDCl}_{3}$
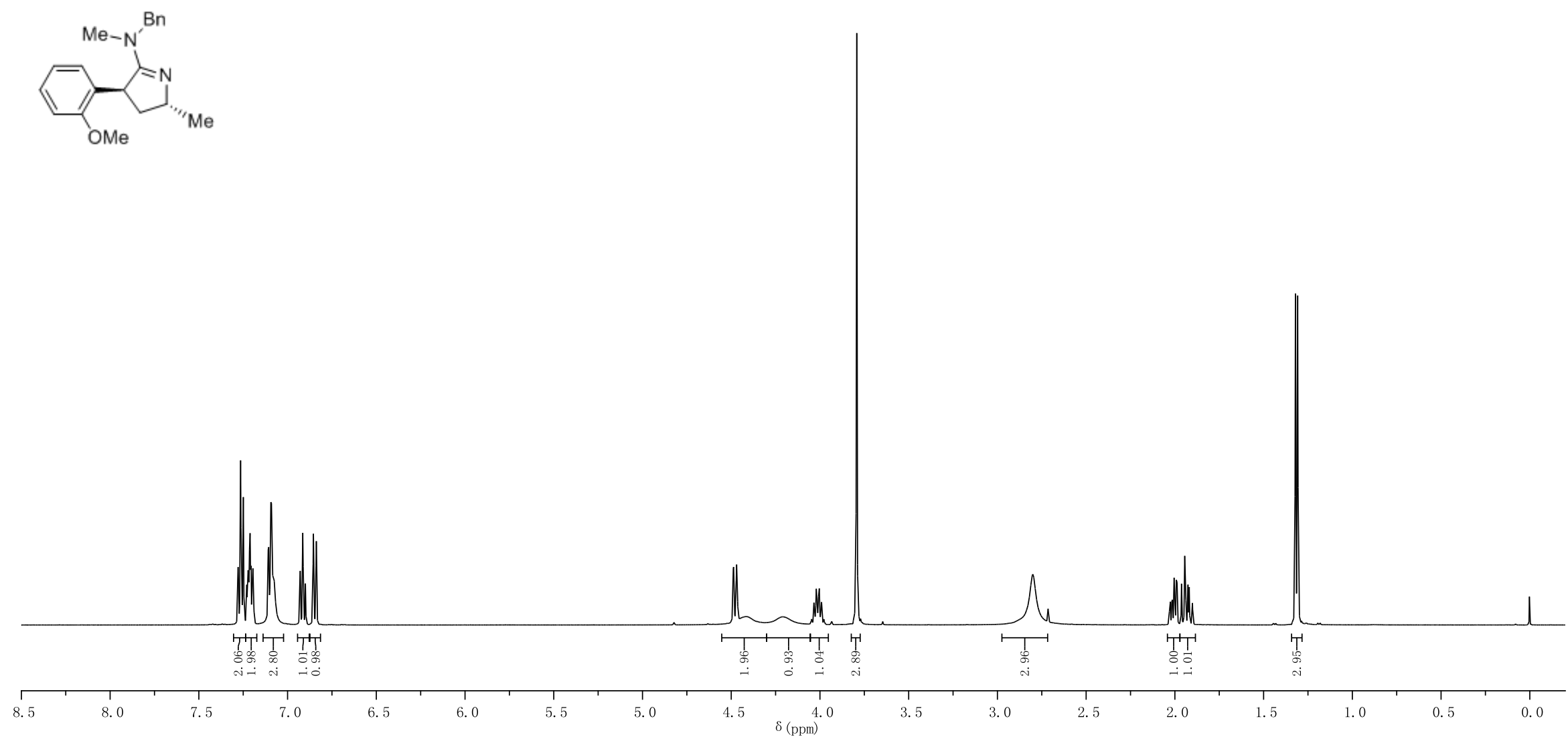


\section{Compound ( \pm )-3la}

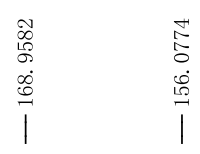

${ }^{13} \mathrm{C}\left\{{ }^{1} \mathrm{H}\right\}$ NMR

$125 \mathrm{MHz}, \mathrm{CDCl}_{3}$
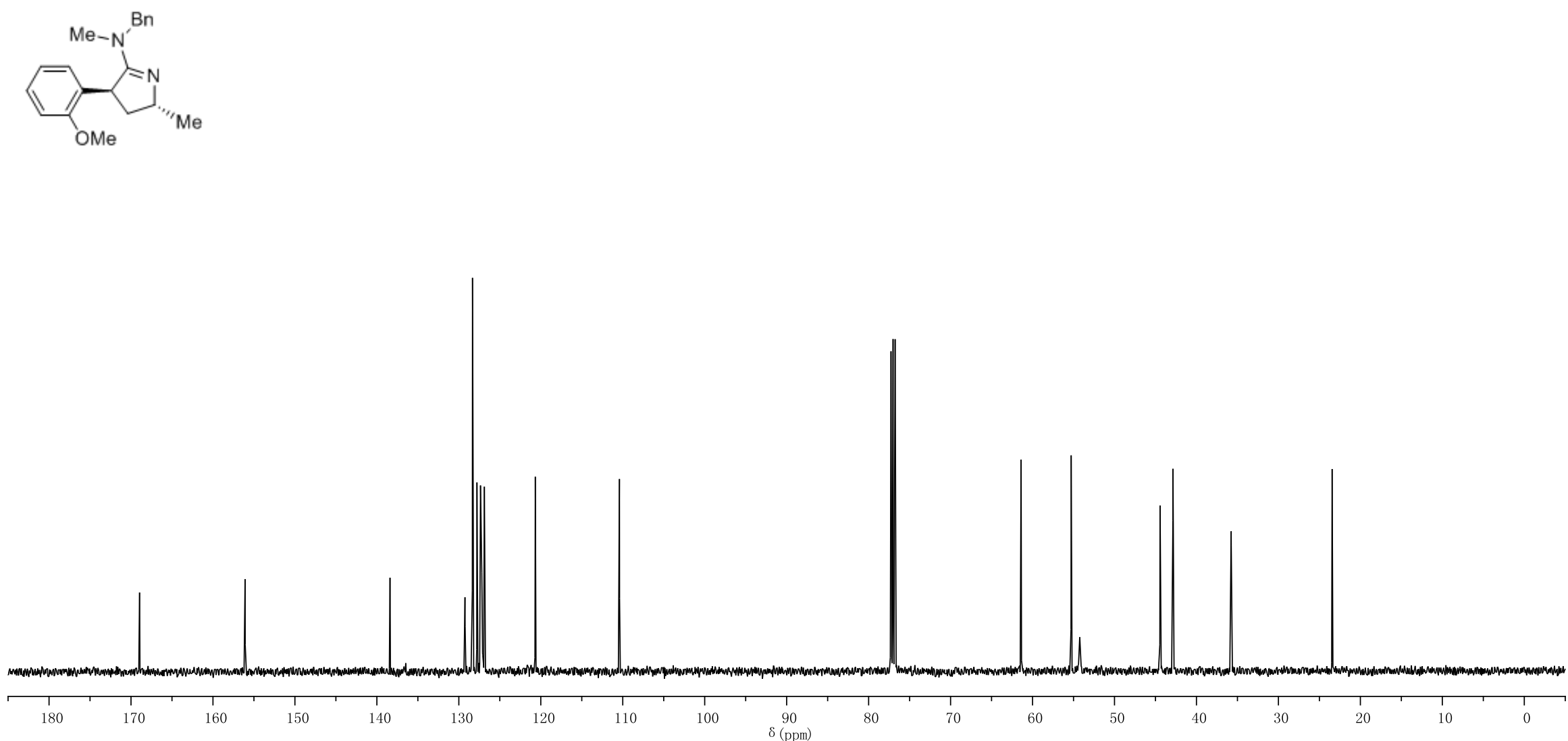


\section{Compound ( \pm )-3ma}

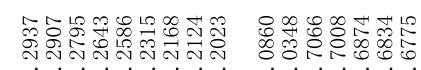

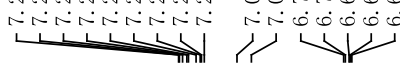

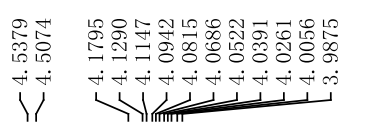

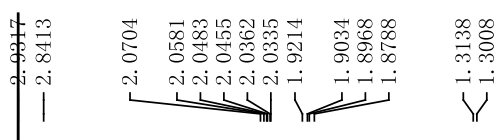

${ }^{1} \mathrm{H}$ NMR

$500 \mathrm{MHz}, \mathrm{CDCl}_{3}$
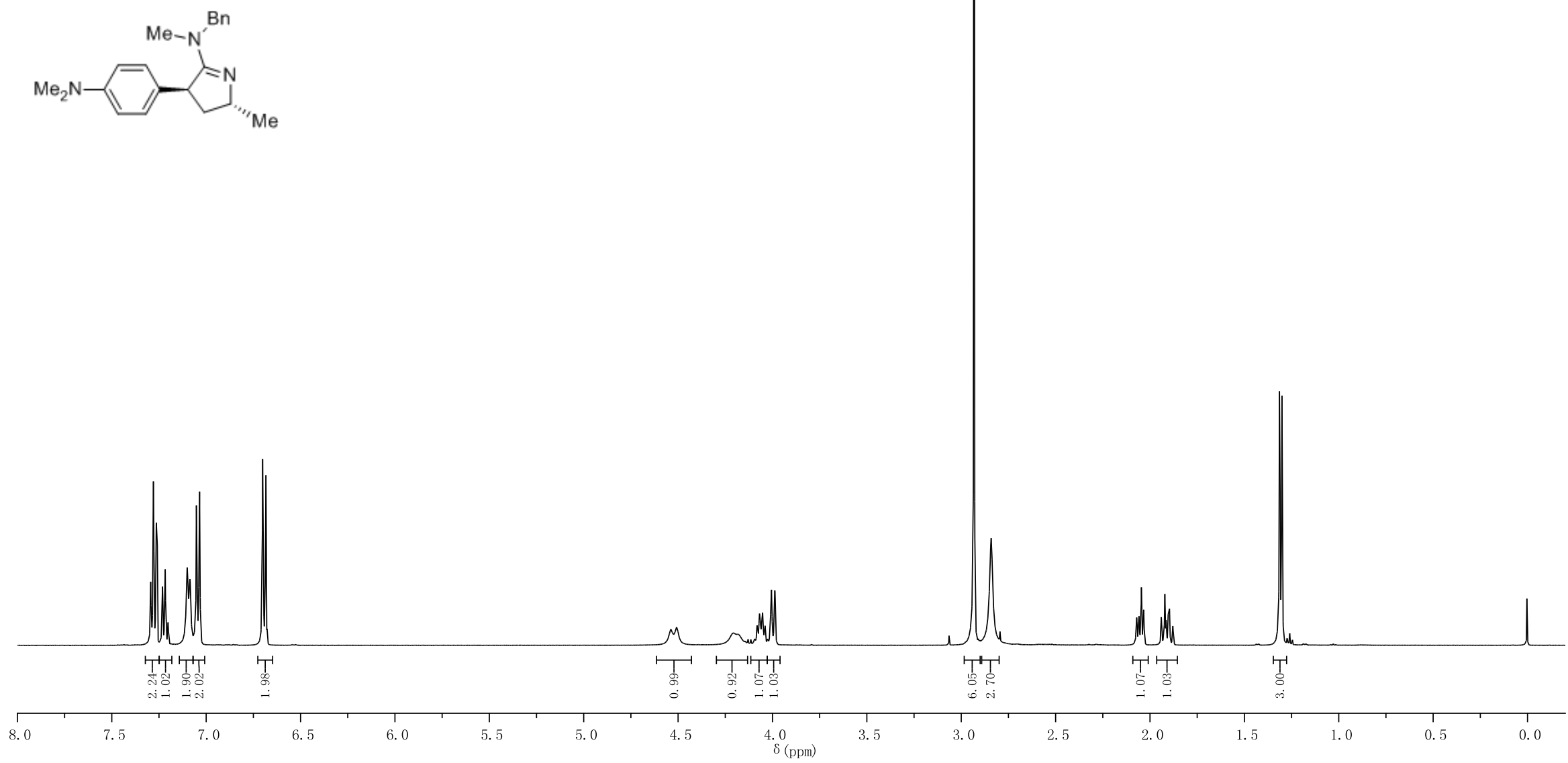


\section{Compound ( \pm )-3ma}

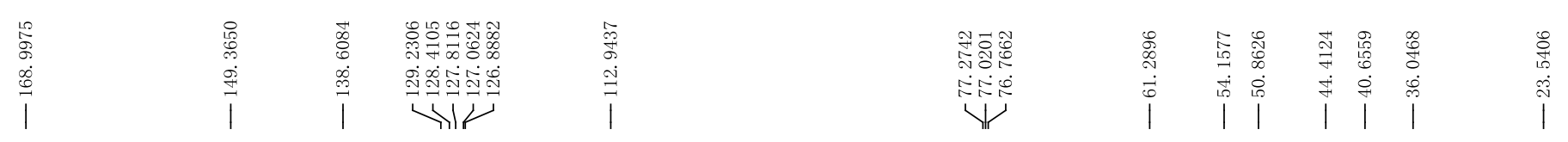

${ }^{13} \mathrm{C}\left\{{ }^{1} \mathrm{H}\right\} \mathrm{NMR}$

$125 \mathrm{MHz}, \mathrm{CDCl}_{3}$
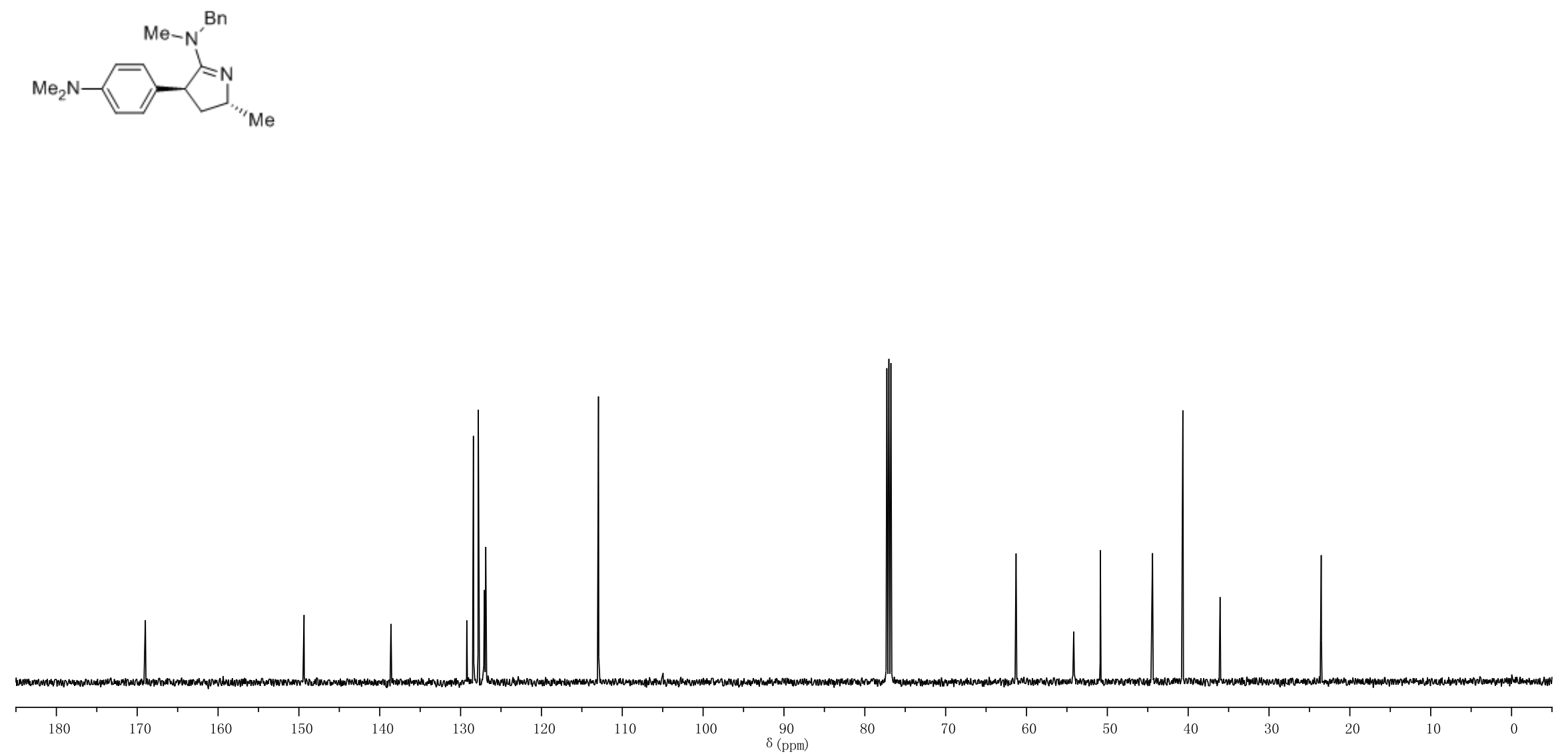


\section{Compound ( \pm )-3na}

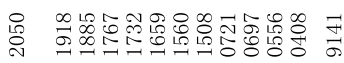

NiNiNiNiNi

${ }^{1} \mathrm{H}$ NMR

$500 \mathrm{MHz}, \mathrm{CDCl}_{3}$
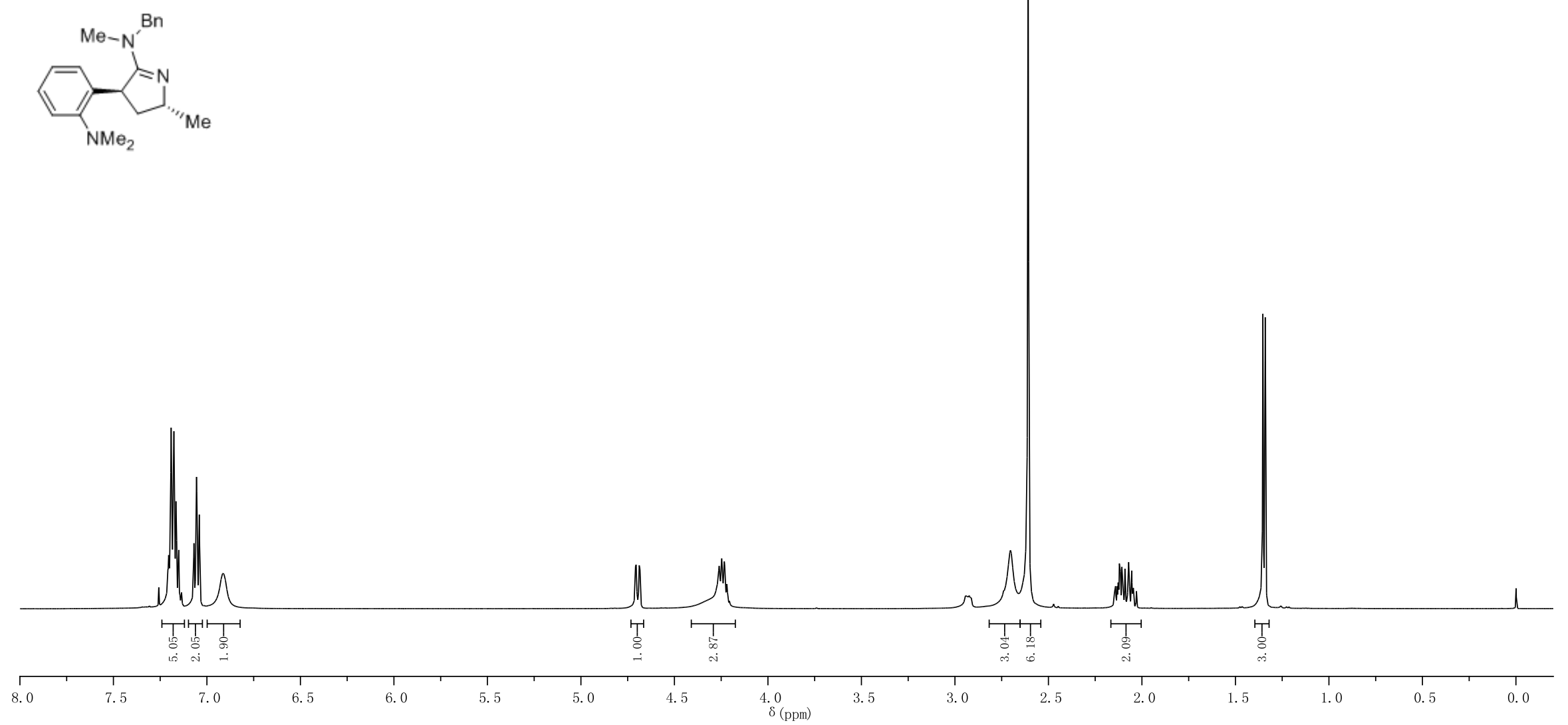


\section{Compound ( \pm )-3na}

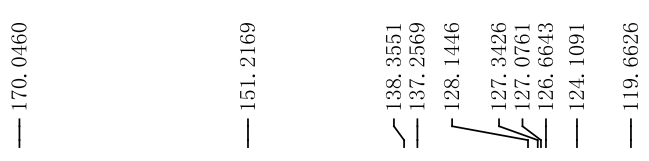

${ }^{13} \mathrm{C}\left\{{ }^{1} \mathrm{H}\right\}$ NMR

$125 \mathrm{MHz}, \mathrm{CDCl}_{3}$
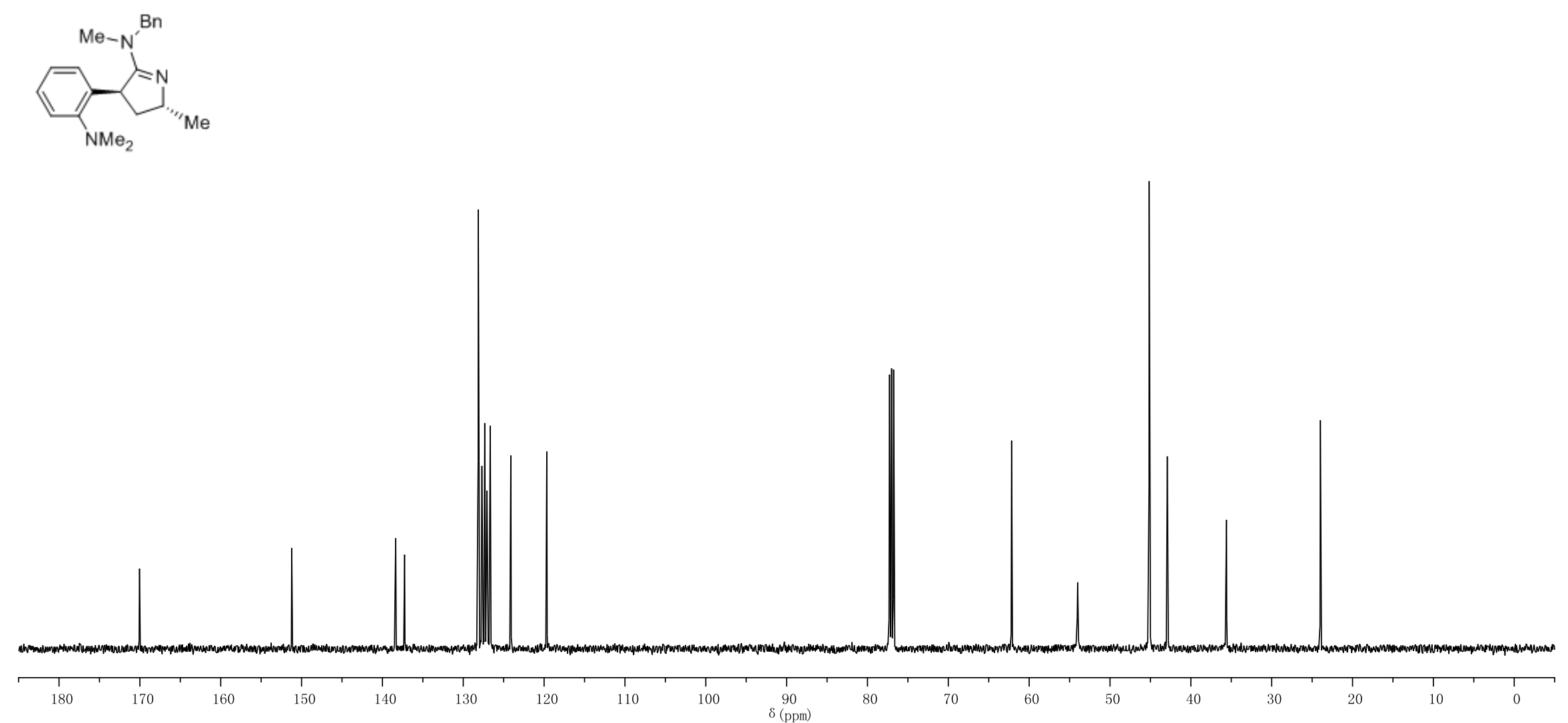


\section{Compound ( \pm )-3oa}

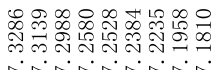

些供

${ }^{1} \mathrm{H}$ NMR

$500 \mathrm{MHz}, \mathrm{CDCl}_{3}$

(ne-ne
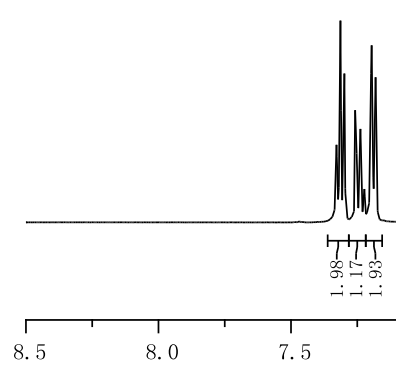

Th
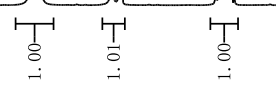

$\delta(\mathrm{ppm})$ 


\section{Compound ( \pm )-3oa}

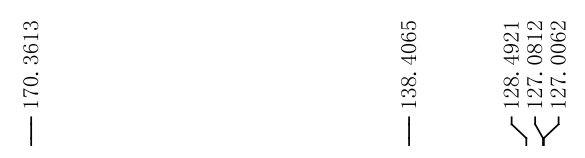

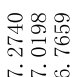

${ }^{13} \mathrm{C}\left\{{ }^{1} \mathrm{H}\right\}$ NMR

$125 \mathrm{MHz}, \mathrm{CDCl}_{3}$
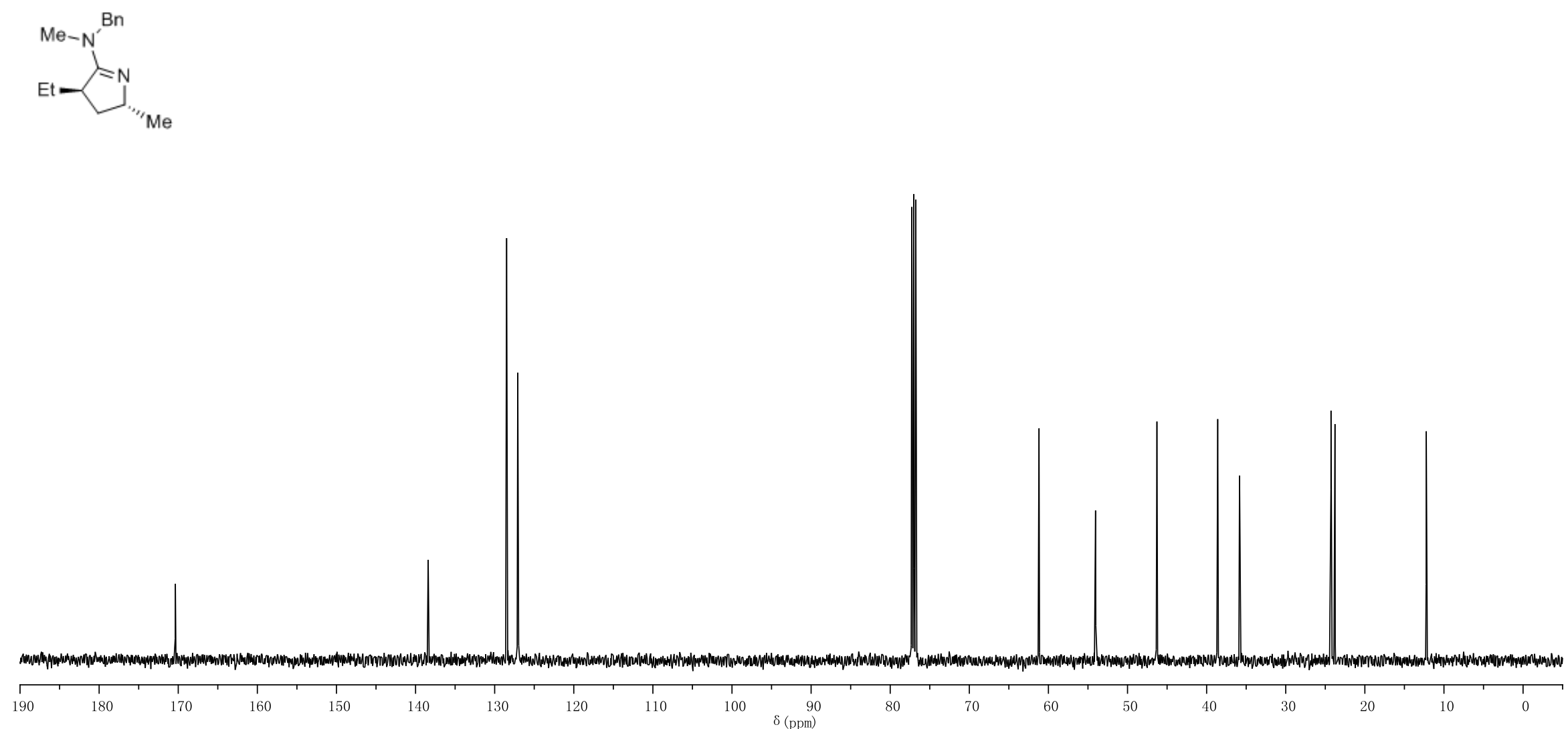


\section{Compound ( \pm )-3pa}

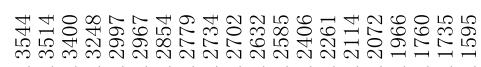

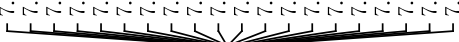

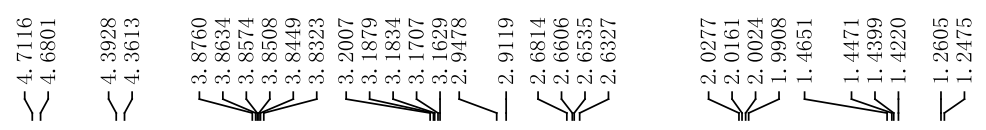

$500 \mathrm{MHz}, \mathrm{CDCl}_{3}$

$$
\text { Me- }
$$

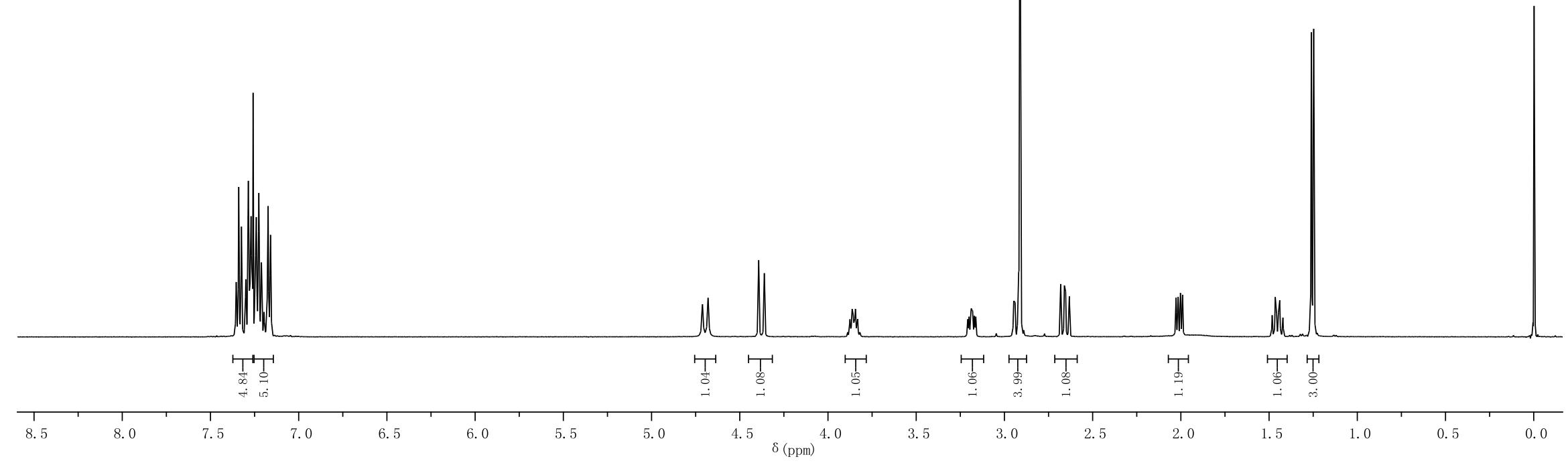




\section{Compound ( \pm$)-3 p a$}

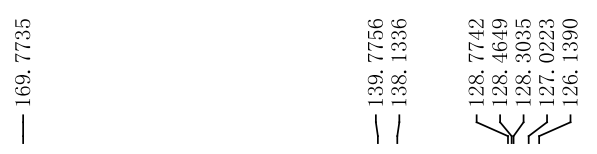

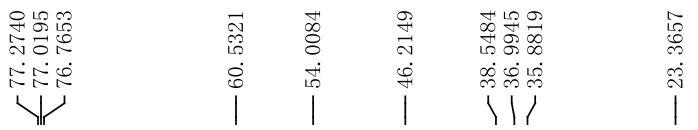

${ }^{13} \mathrm{C}\left\{{ }^{1} \mathrm{H}\right\}$ NMR

$125 \mathrm{MHz}, \mathrm{CDCl}_{3}$

(ne-N"

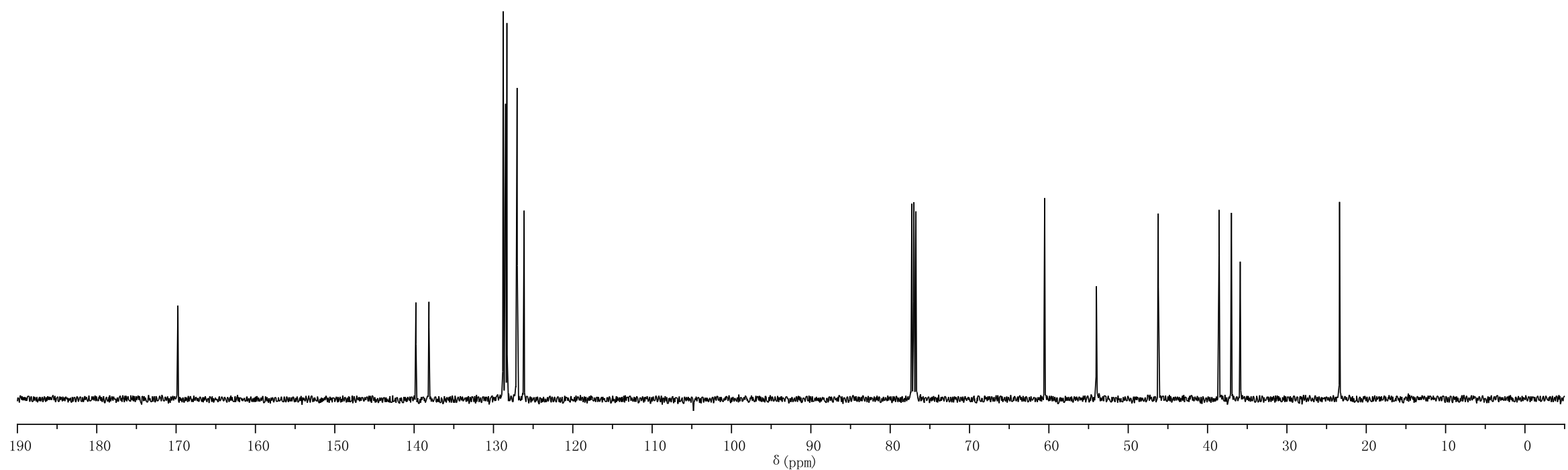




\section{Compound ( \pm )-3qa}

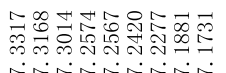

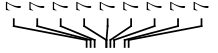

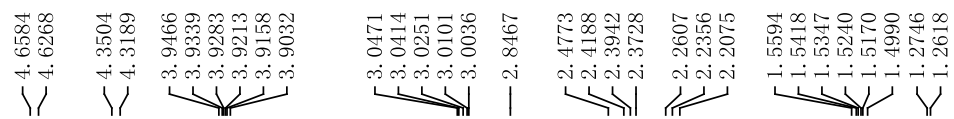

${ }^{1} \mathrm{H}$ NMR

$500 \mathrm{MHz}, \mathrm{CDCl}_{3}$
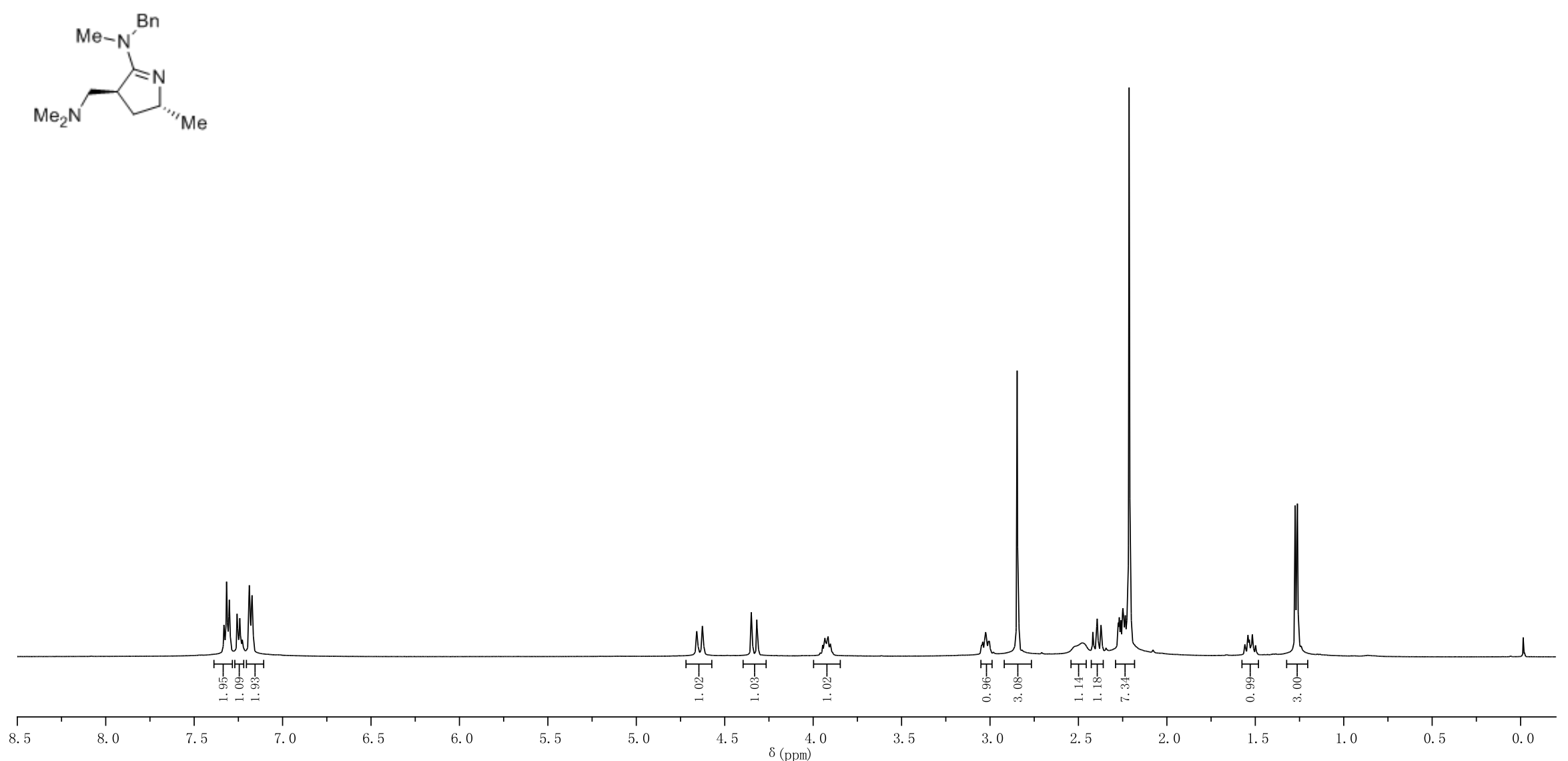
Compound ( \pm )-3qa

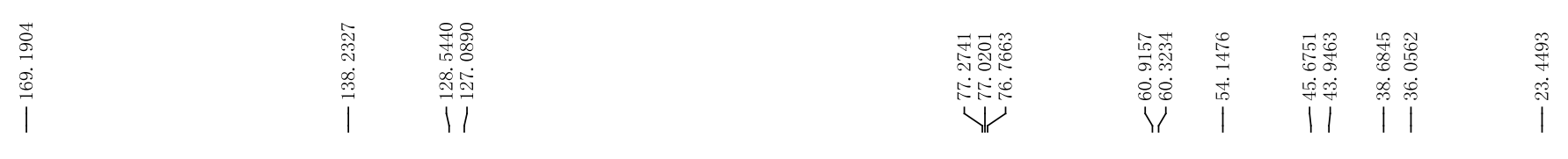

${ }^{13} \mathrm{C}\left\{{ }^{1} \mathrm{H}\right\}$ NMR

$125 \mathrm{MHz}, \mathrm{CDCl}_{3}$
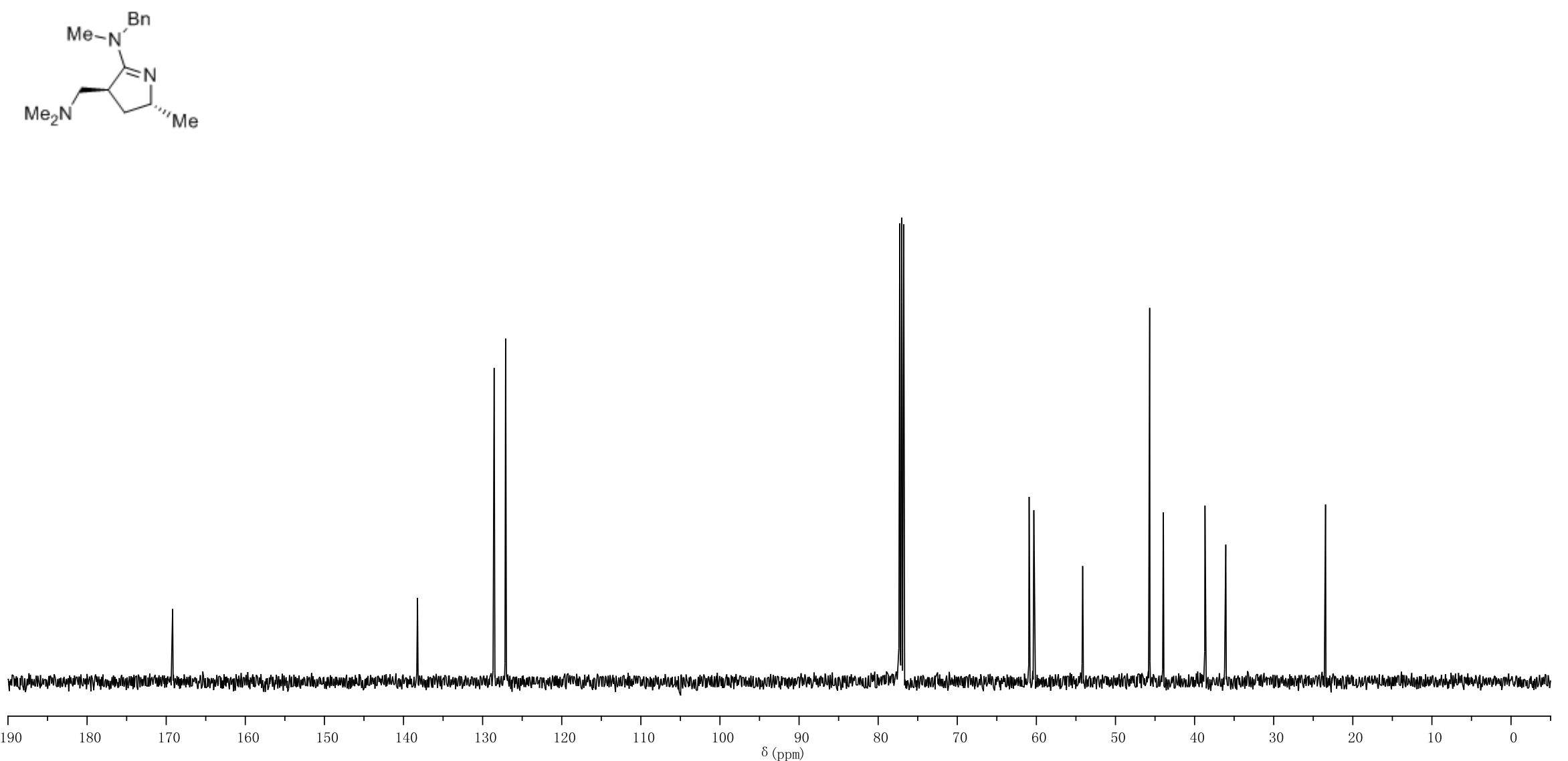


\section{Compound ( \pm )-3ra}

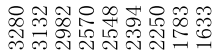

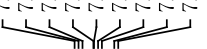

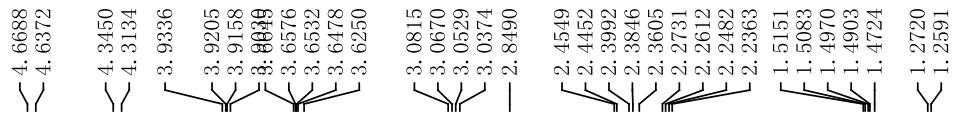

${ }^{1} \mathrm{H}$ NMR

$500 \mathrm{MHz}, \mathrm{CDCl}_{3}$
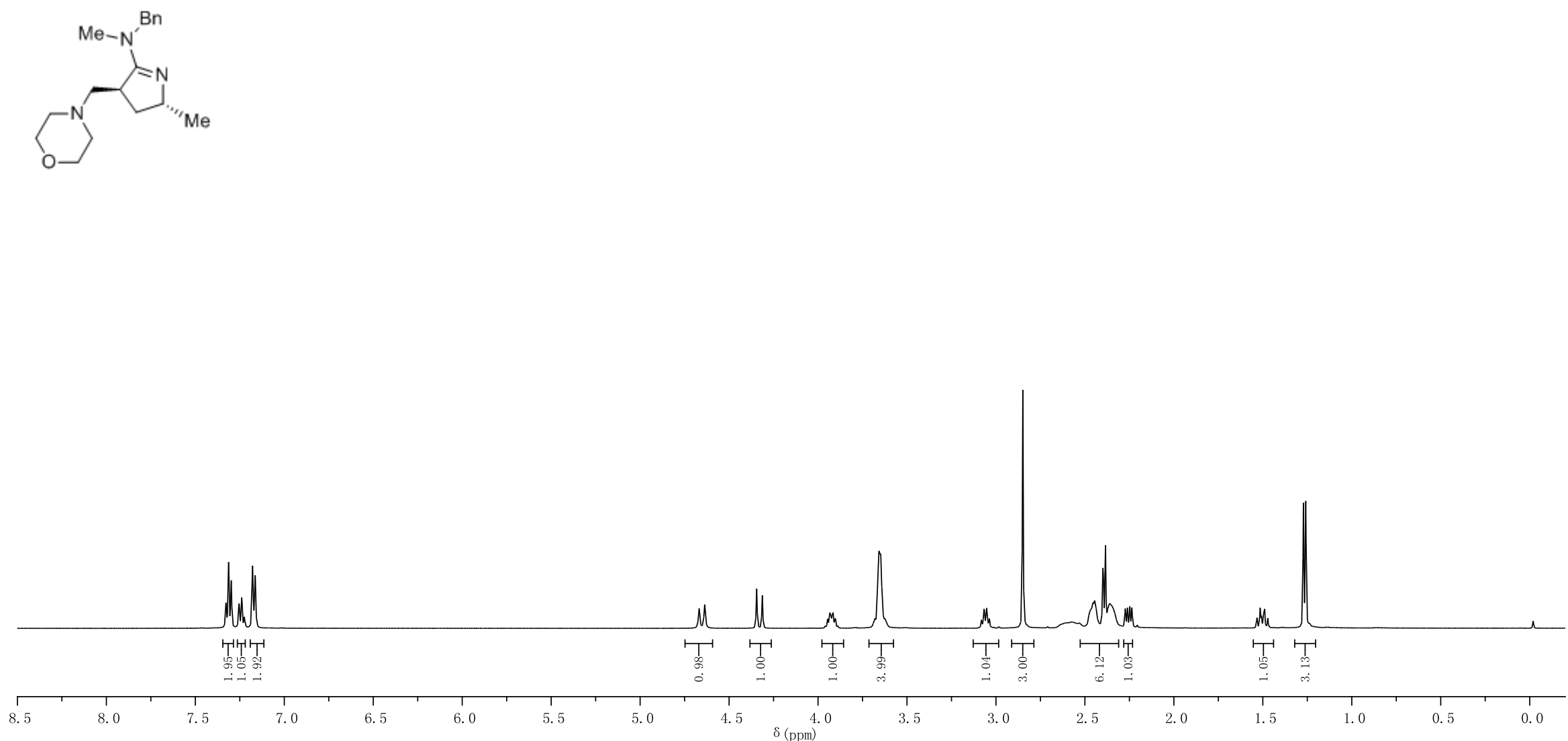


\section{Compound ( \pm )-3ra}

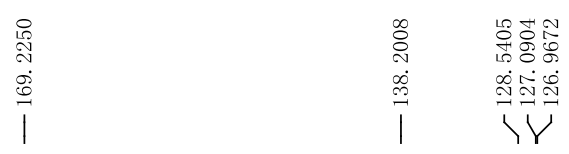

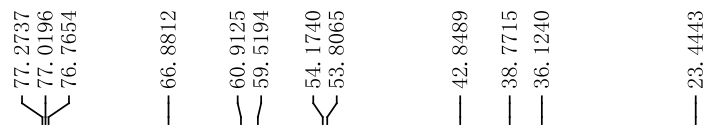

${ }^{13} \mathrm{C}\left\{{ }^{1} \mathrm{H}\right\}$ NMR

$125 \mathrm{MHz}, \mathrm{CDCl}_{3}$
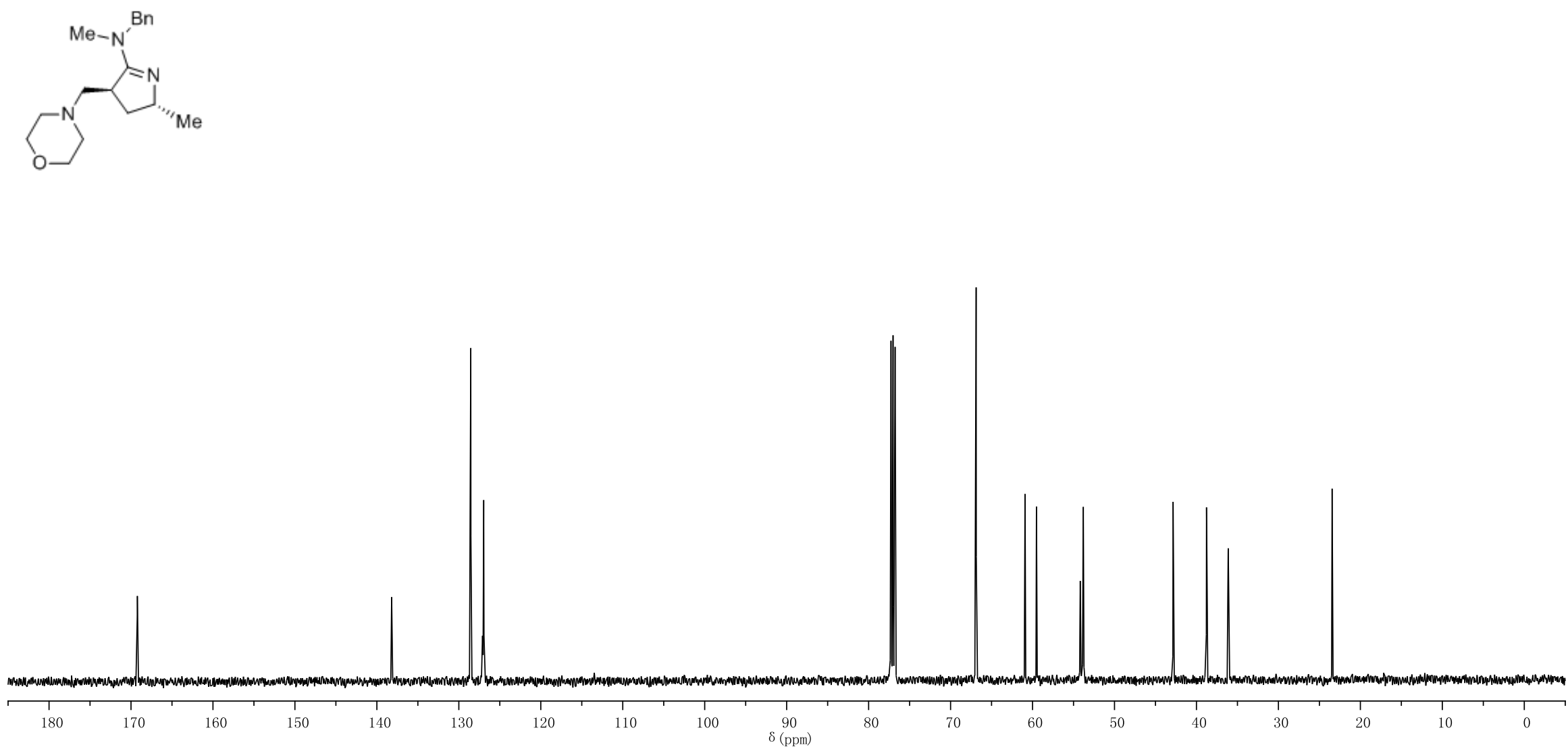


\section{Compound ( \pm )-3sa}

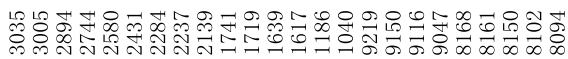

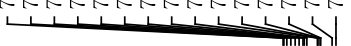

${ }^{1} \mathrm{H}$ NMR

$500 \mathrm{MHz}, \mathrm{CDCl}_{3}$
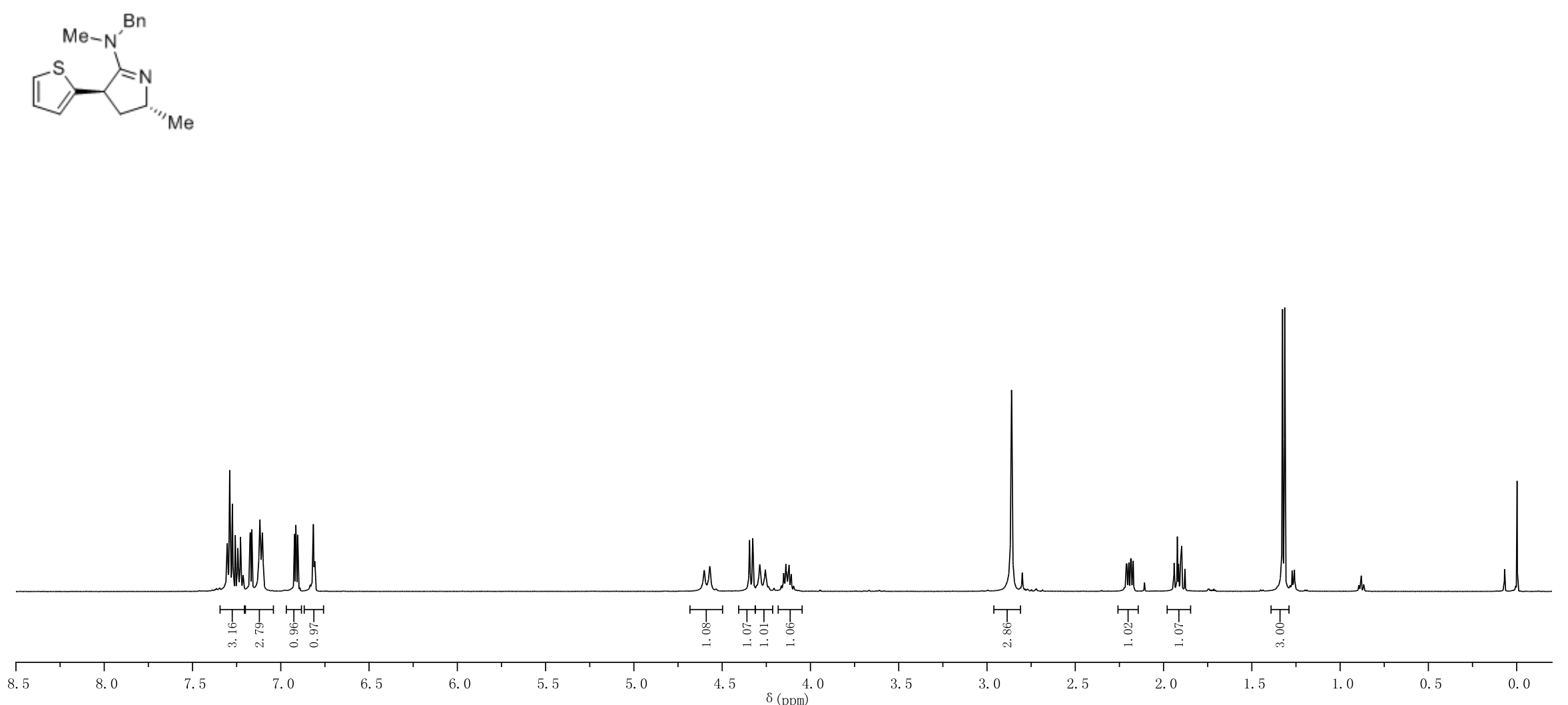


\section{Compound ( \pm$)$-3sa}

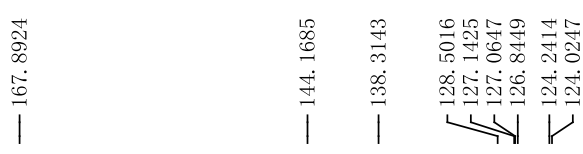

${ }^{13} \mathrm{C}\left\{{ }^{1} \mathrm{H}\right\}$ NMR

$125 \mathrm{MHz}, \mathrm{CDCl}_{3}$
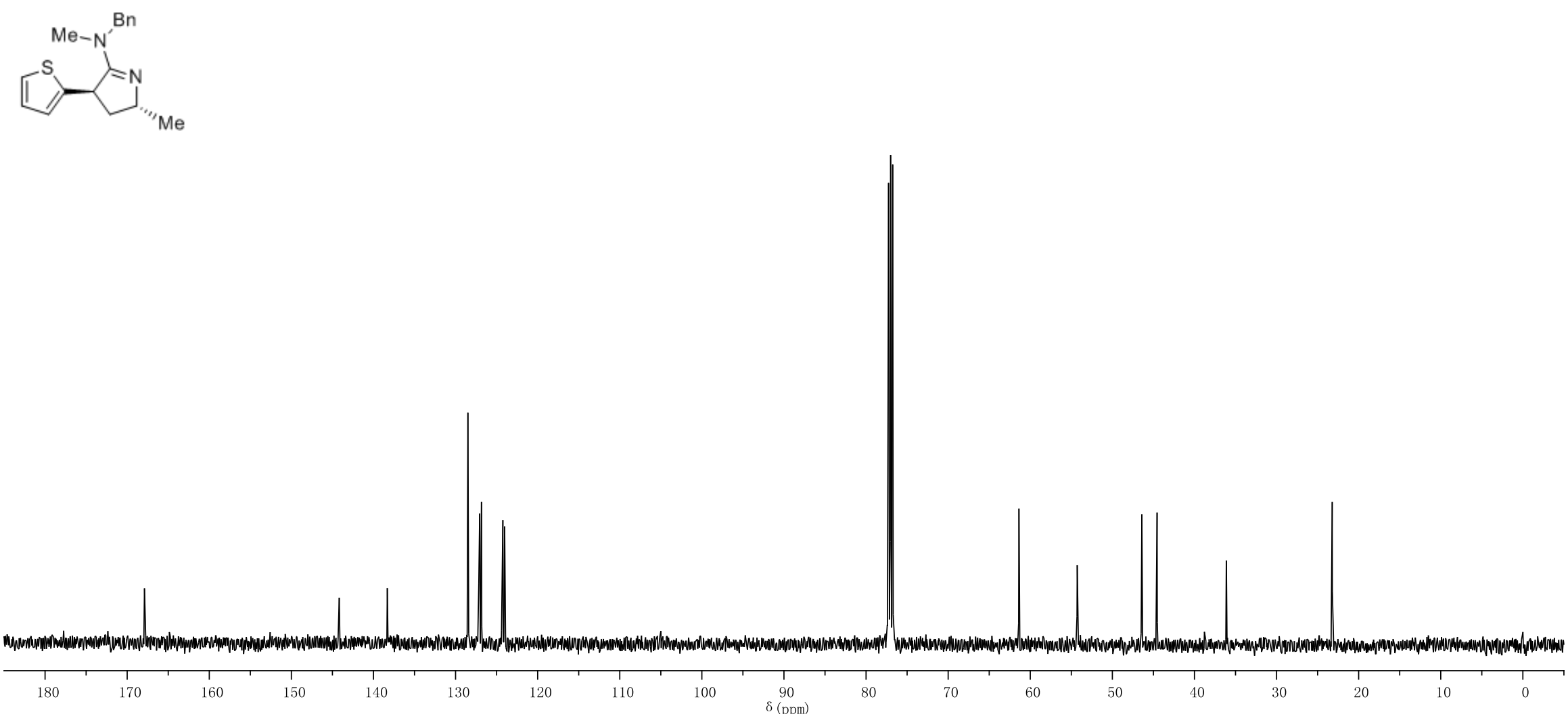


\section{Compound ( \pm )-3ta}

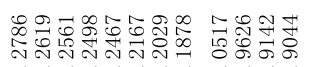

ninisini

${ }^{1} \mathrm{H}$ NMR

$500 \mathrm{MHz}, \mathrm{CDCl}_{3}$
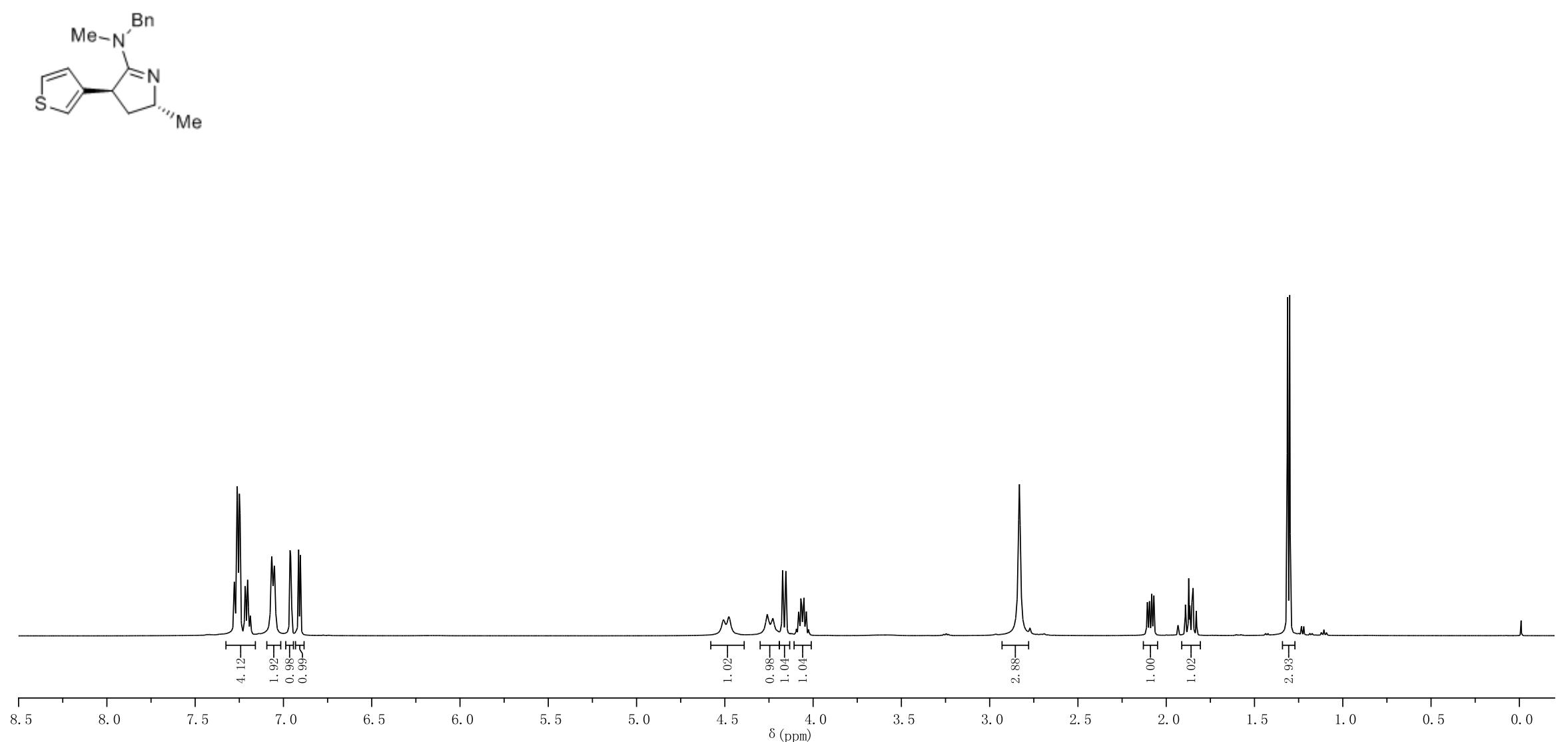


\section{Compound ( \pm )-3ta}

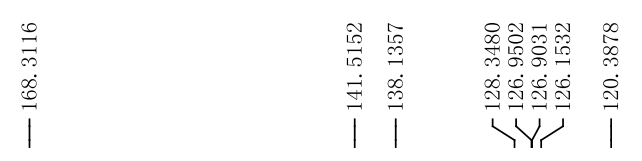

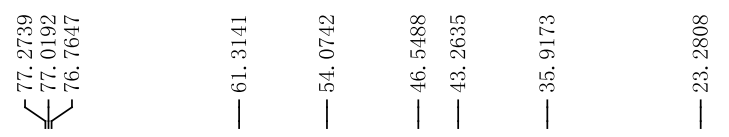

${ }^{13} \mathrm{C}\left\{{ }^{1} \mathrm{H}\right\}$ NMR

$125 \mathrm{MHz}, \mathrm{CDCl}_{3}$
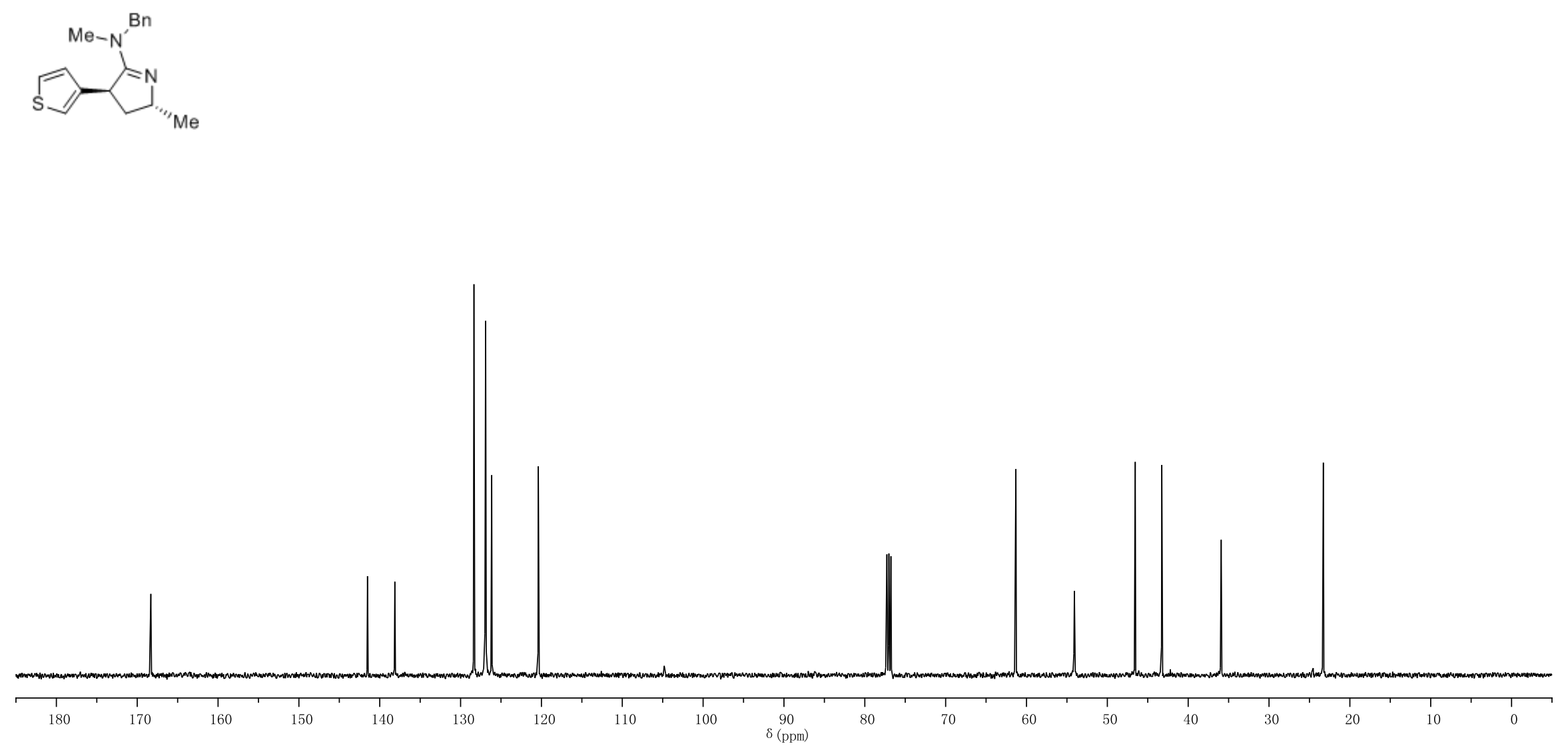


\section{Compound ( \pm )-3wa}

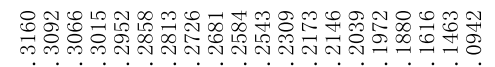

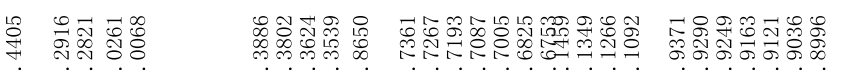

i

${ }^{1} \mathrm{H}$ NMR

$500 \mathrm{MHz}, \mathrm{CDCl}_{3}$
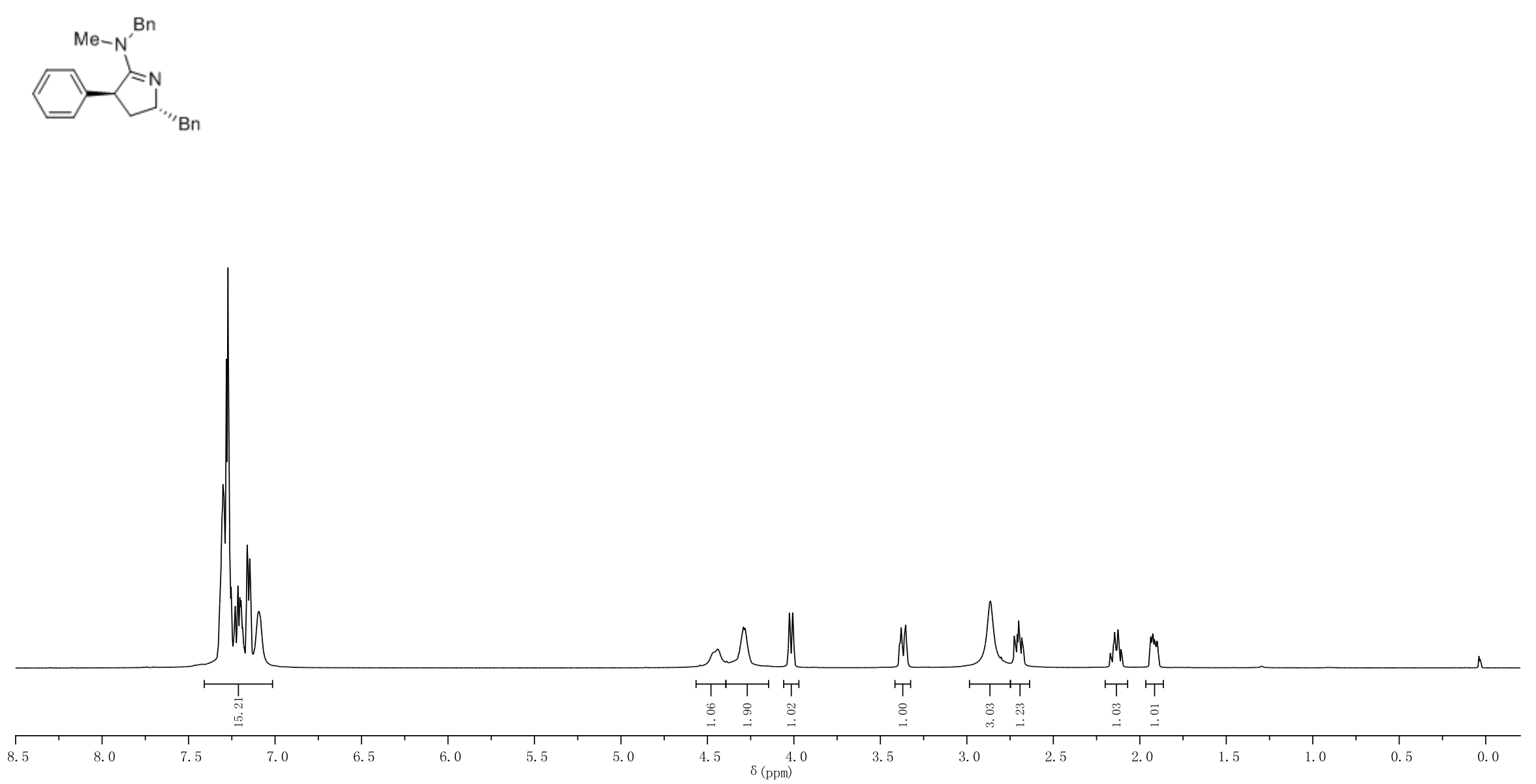


\section{Compound ( \pm )-3wa}

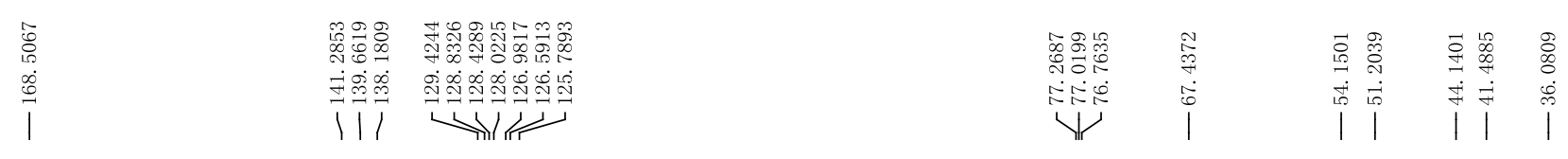

${ }^{13} \mathrm{C}\left\{{ }^{1} \mathrm{H}\right\}$ NMR

$125 \mathrm{MHz}, \mathrm{CDCl}_{3}$
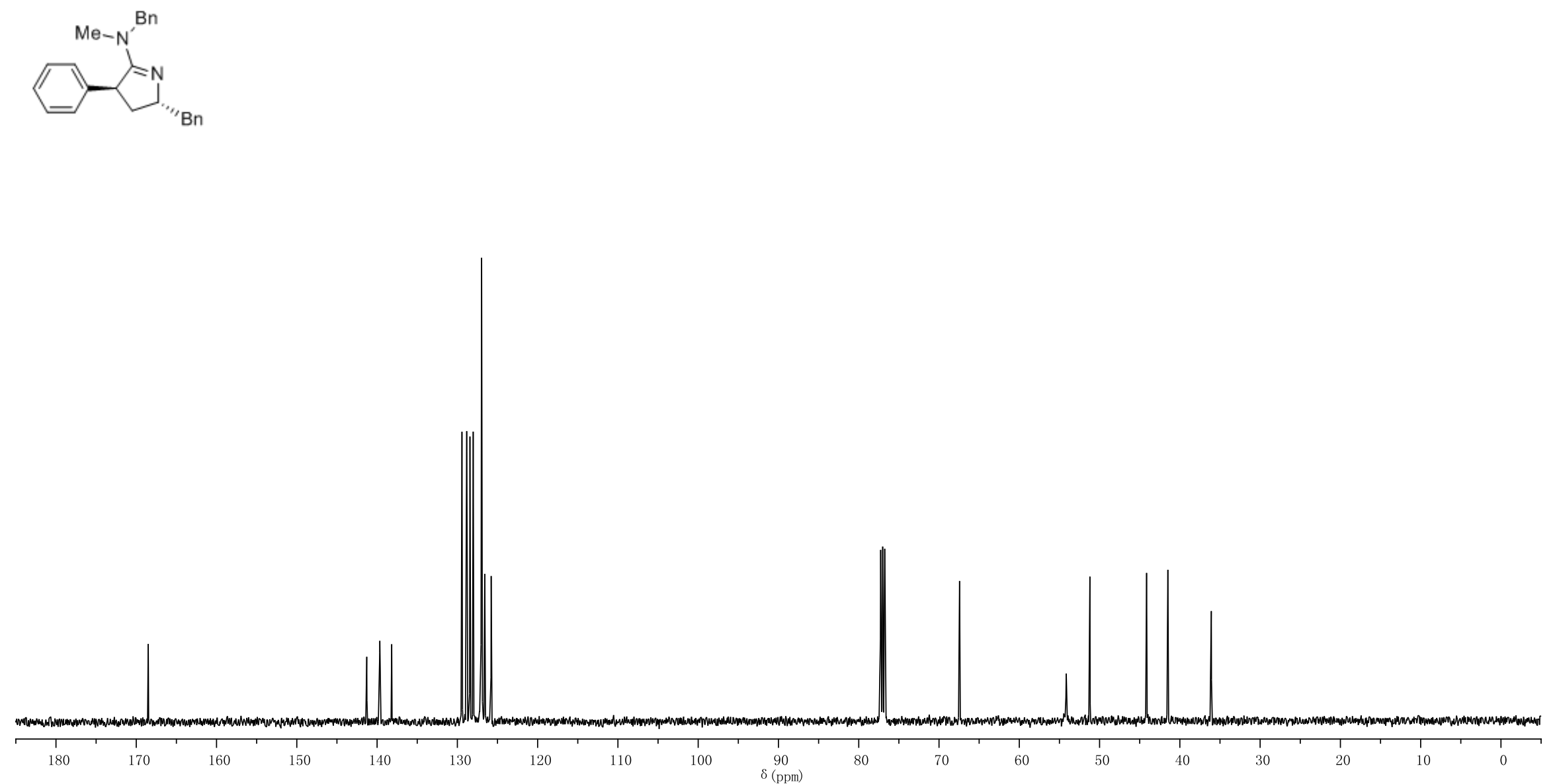


\section{Compound ( \pm )-3xa}

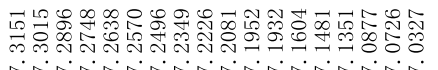

inisininisinisinis

${ }^{1} \mathrm{H}$ NMR

$500 \mathrm{MHz}, \mathrm{CDCl}_{3}$
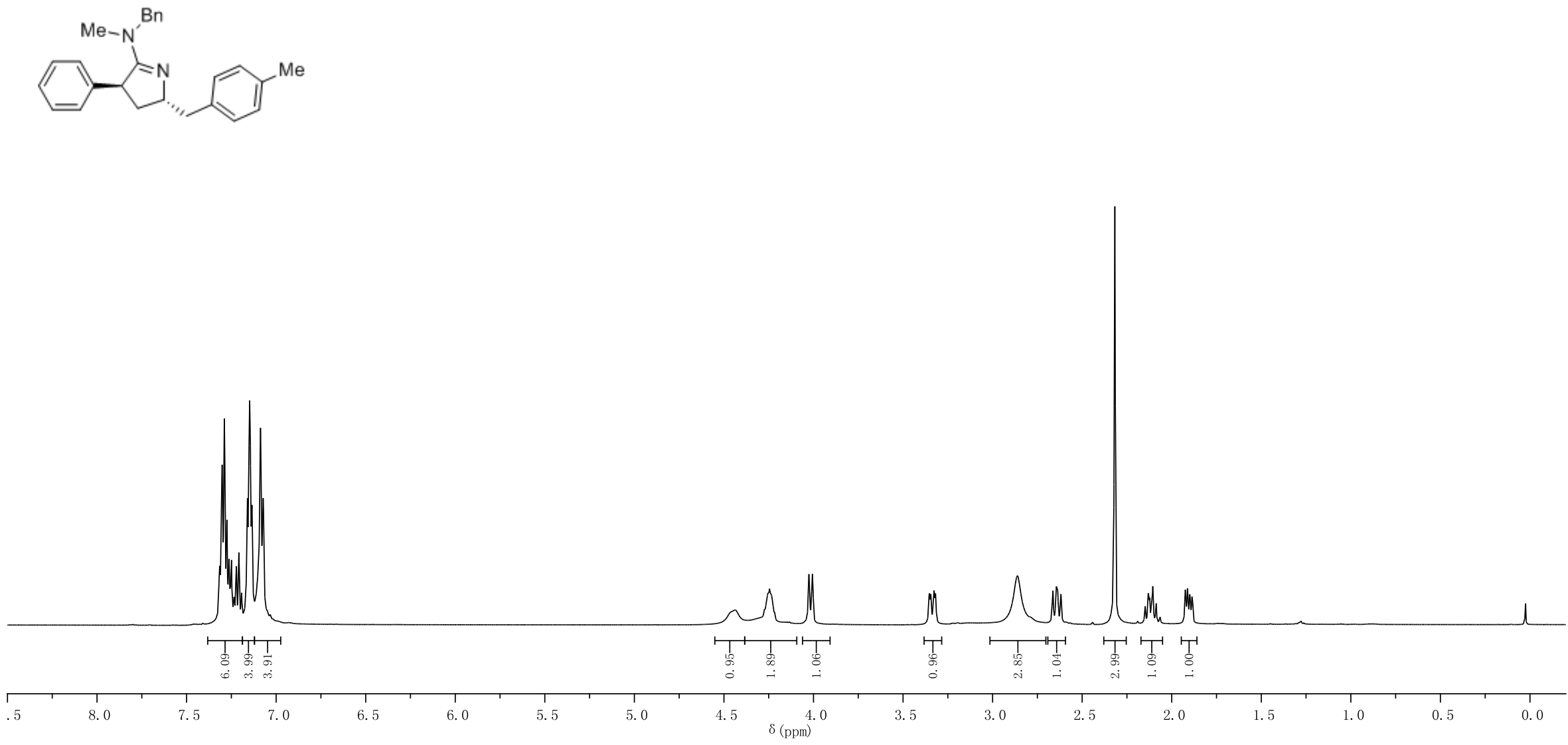


\section{Compound ( \pm )-3xa}

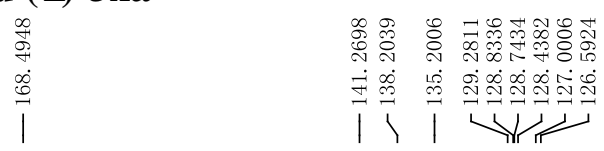

${ }^{13} \mathrm{C}\left\{{ }^{1} \mathrm{H}\right\}$ NMR

$125 \mathrm{MHz}, \mathrm{CDCl}_{3}$
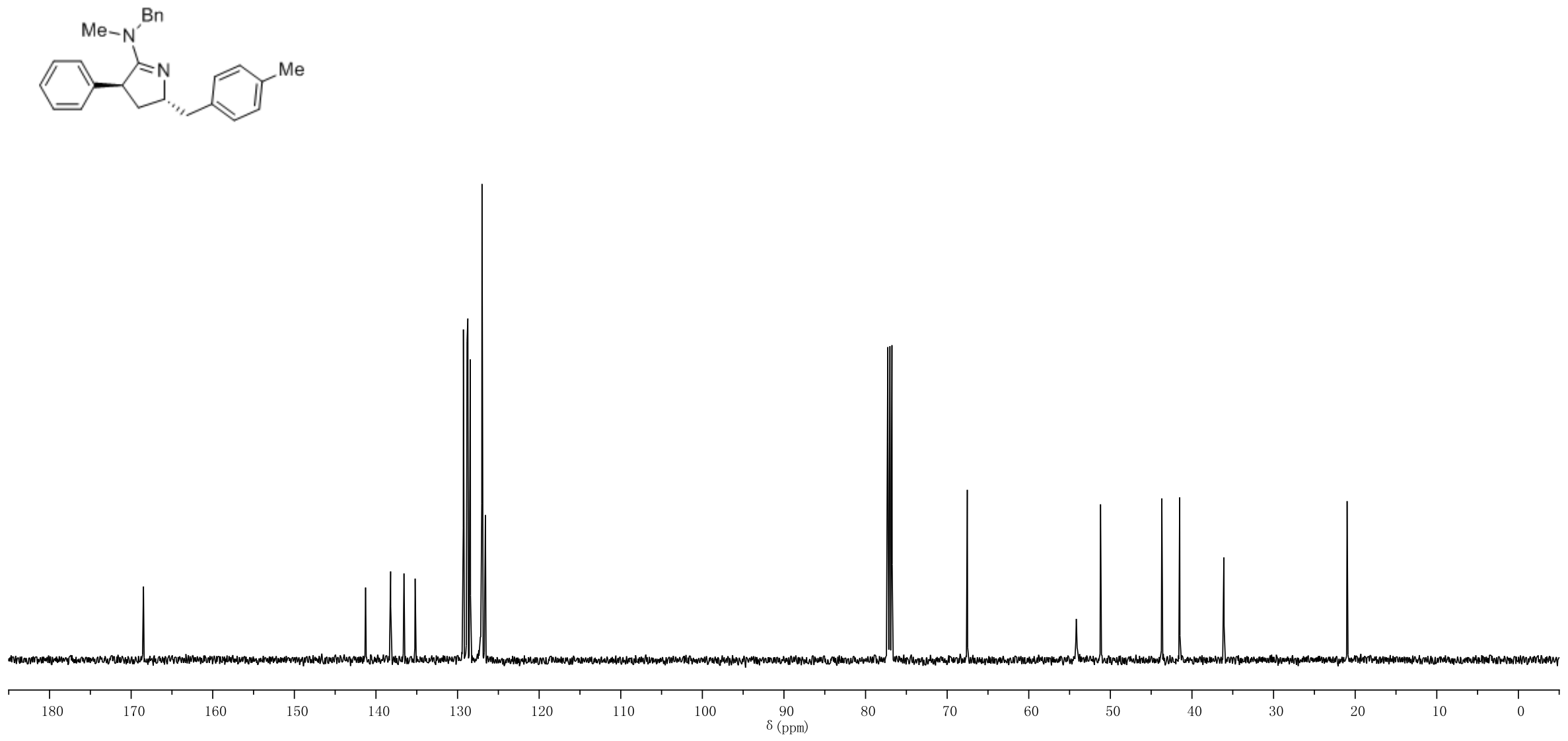


\section{Compound ( \pm )-3ya}

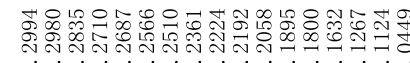

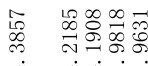

i

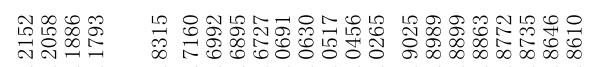

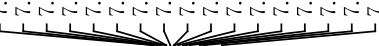

${ }^{1} \mathrm{H}$ NMR

$500 \mathrm{MHz}, \mathrm{CDCl}_{3}$
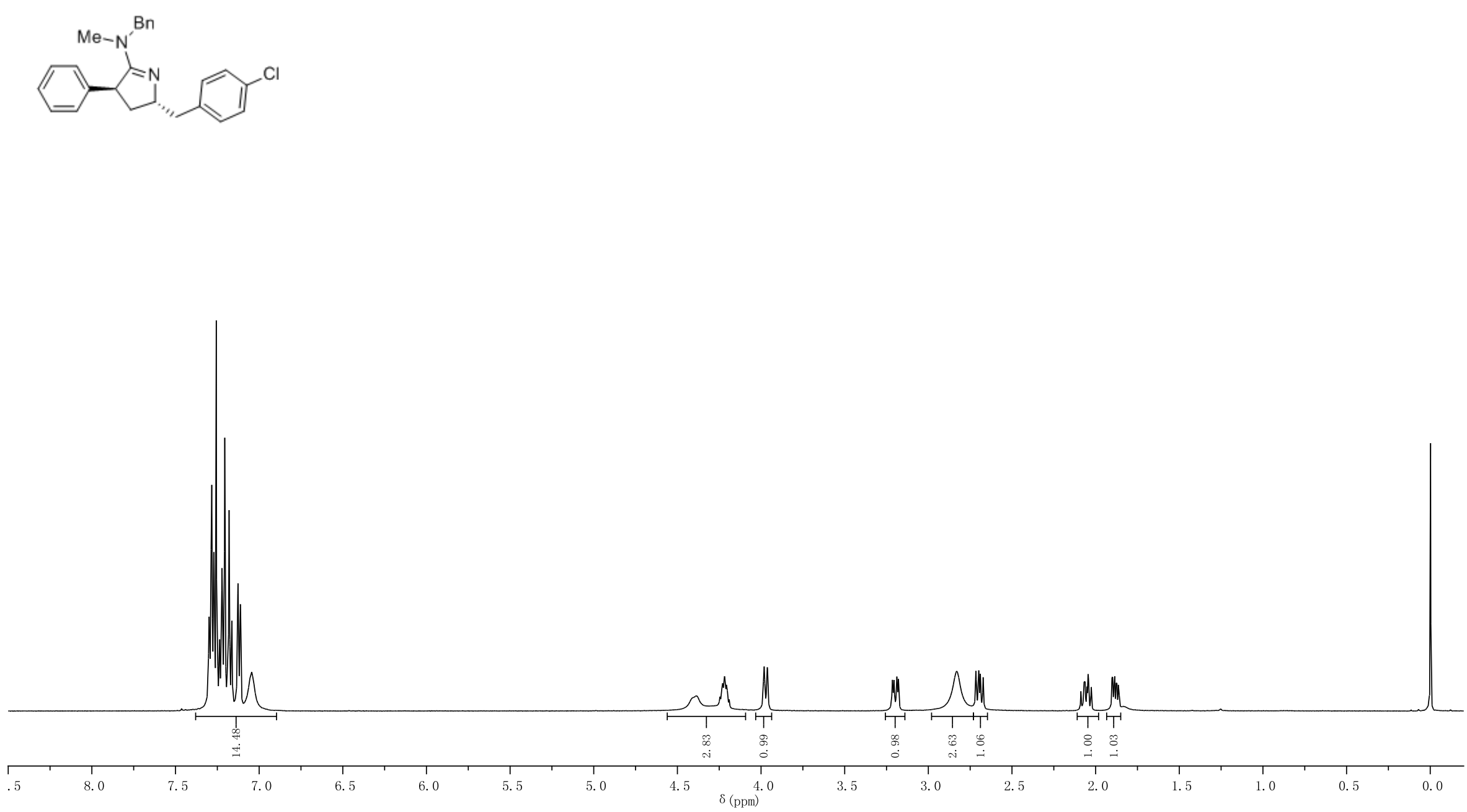


\section{Compound ( \pm )-3ya}

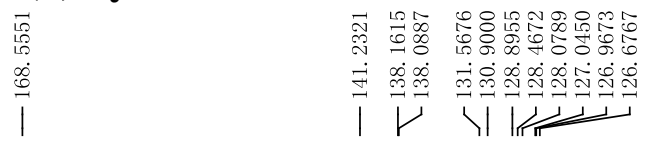

${ }^{13} \mathrm{C}\left\{{ }^{1} \mathrm{H}\right\}$ NMR

$125 \mathrm{MHz}, \mathrm{CDCl}_{3}$
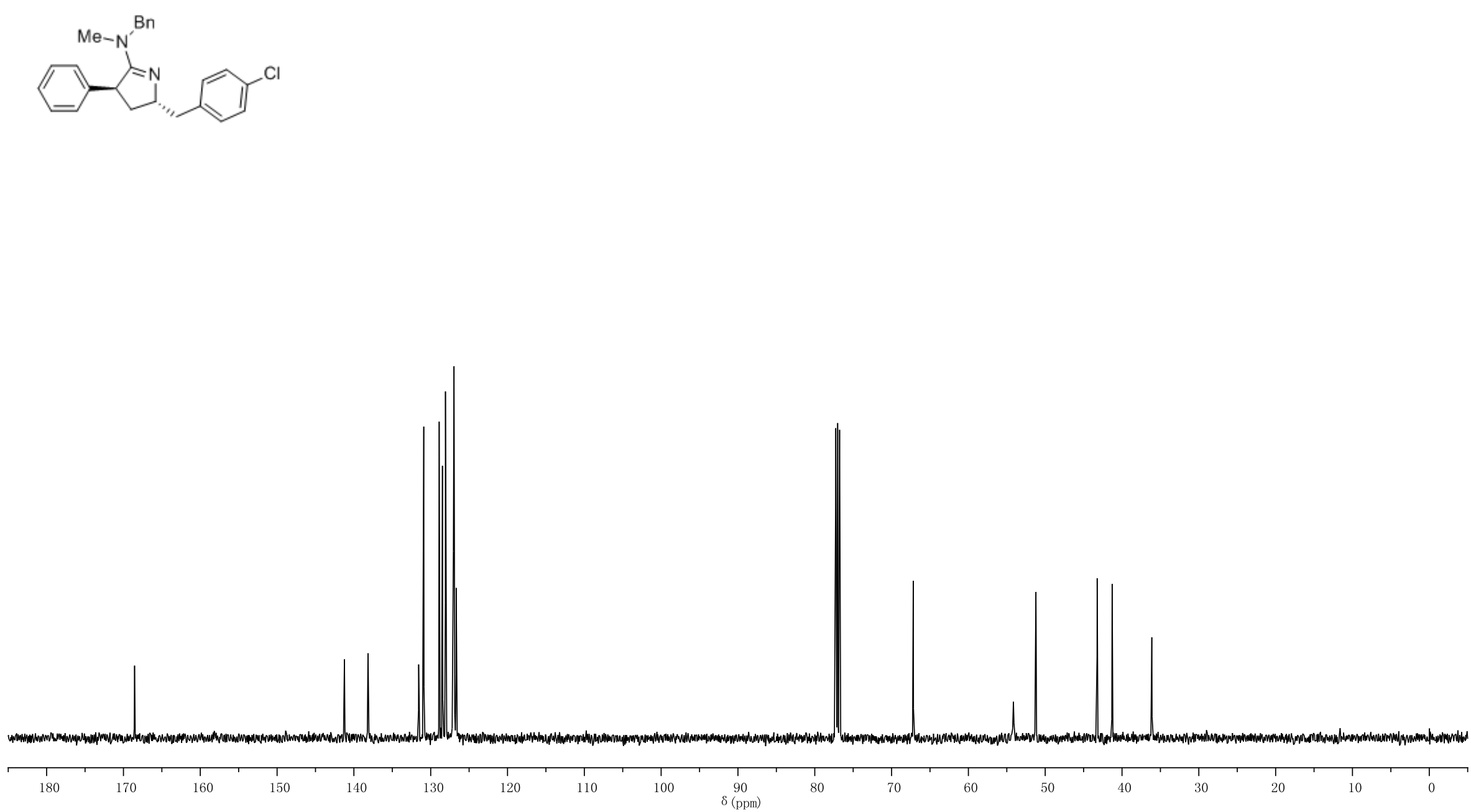


\section{Compound ( \pm )-3za}

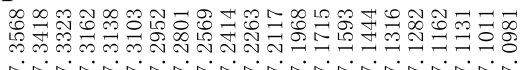

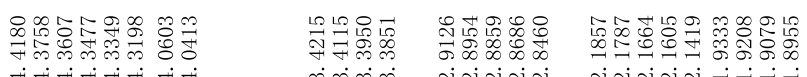

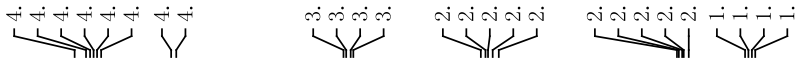

${ }^{1} \mathrm{H}$ NMR

$500 \mathrm{MHz}, \mathrm{CDCl}_{3}$
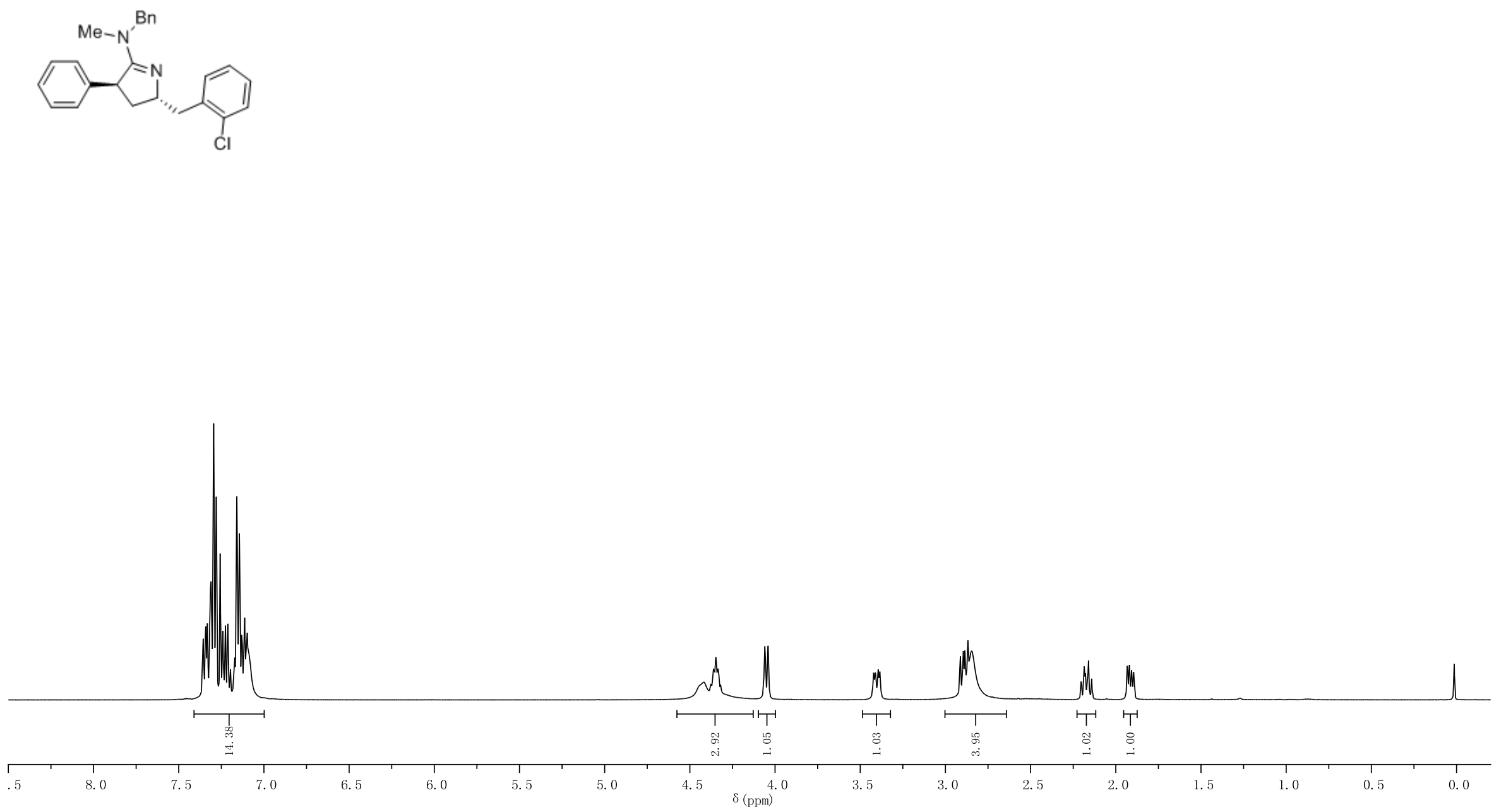


\section{Compound ( \pm )-3za}

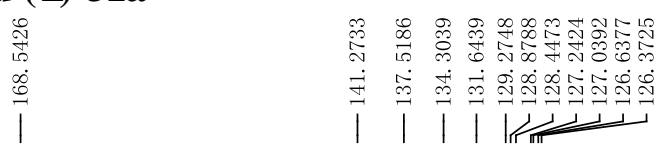

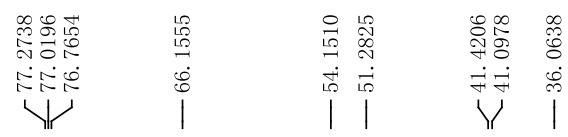

${ }^{13} \mathrm{C}\left\{{ }^{1} \mathrm{H}\right\}$ NMR

$125 \mathrm{MHz}, \mathrm{CDCl}_{3}$
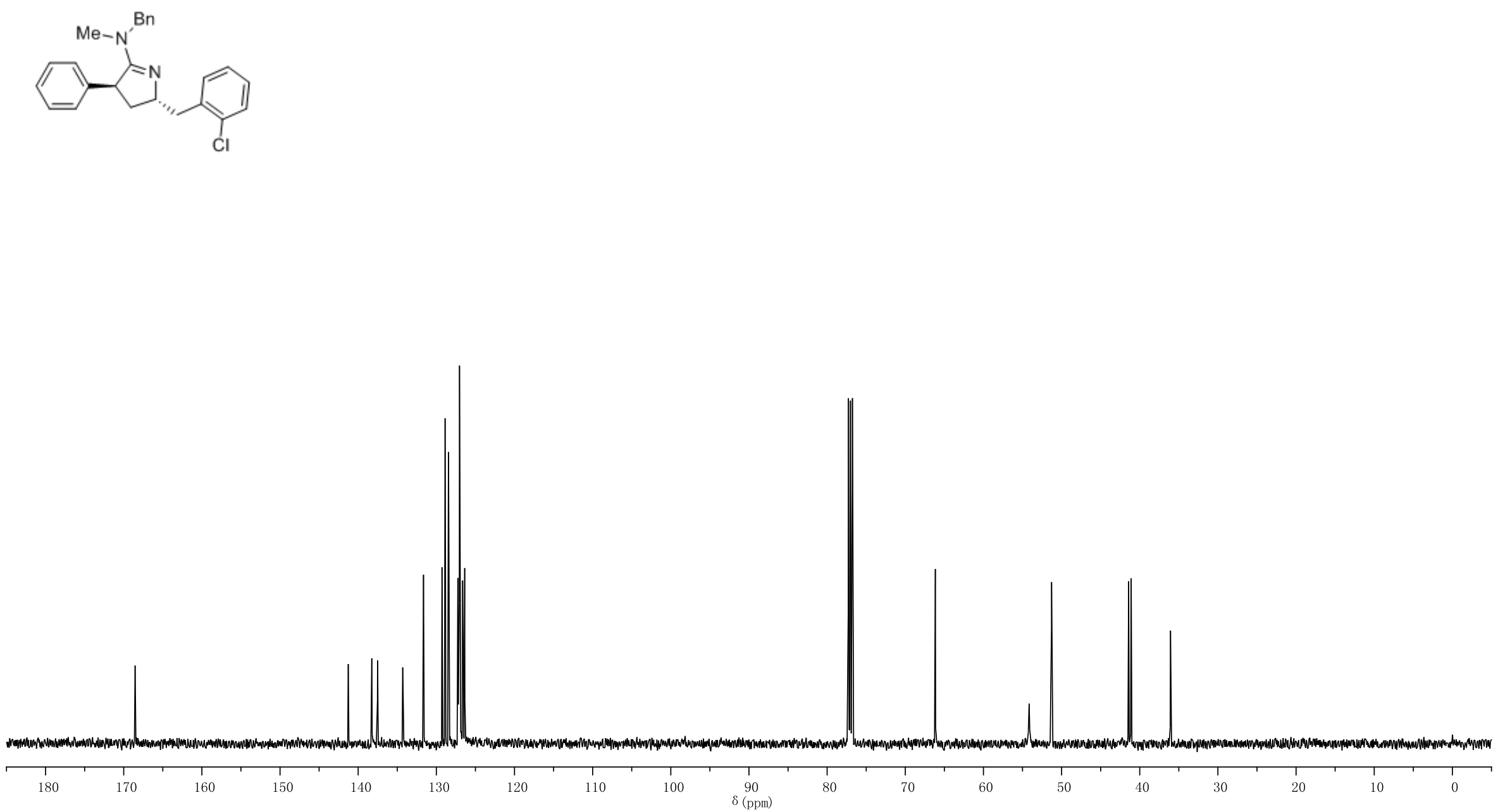


\section{Compound ( \pm )-3zaa}

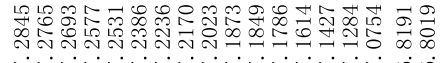

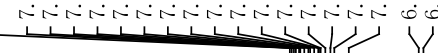

${ }^{1} \mathrm{H}$ NMR

$500 \mathrm{MHz}, \mathrm{CDCl}_{3}$
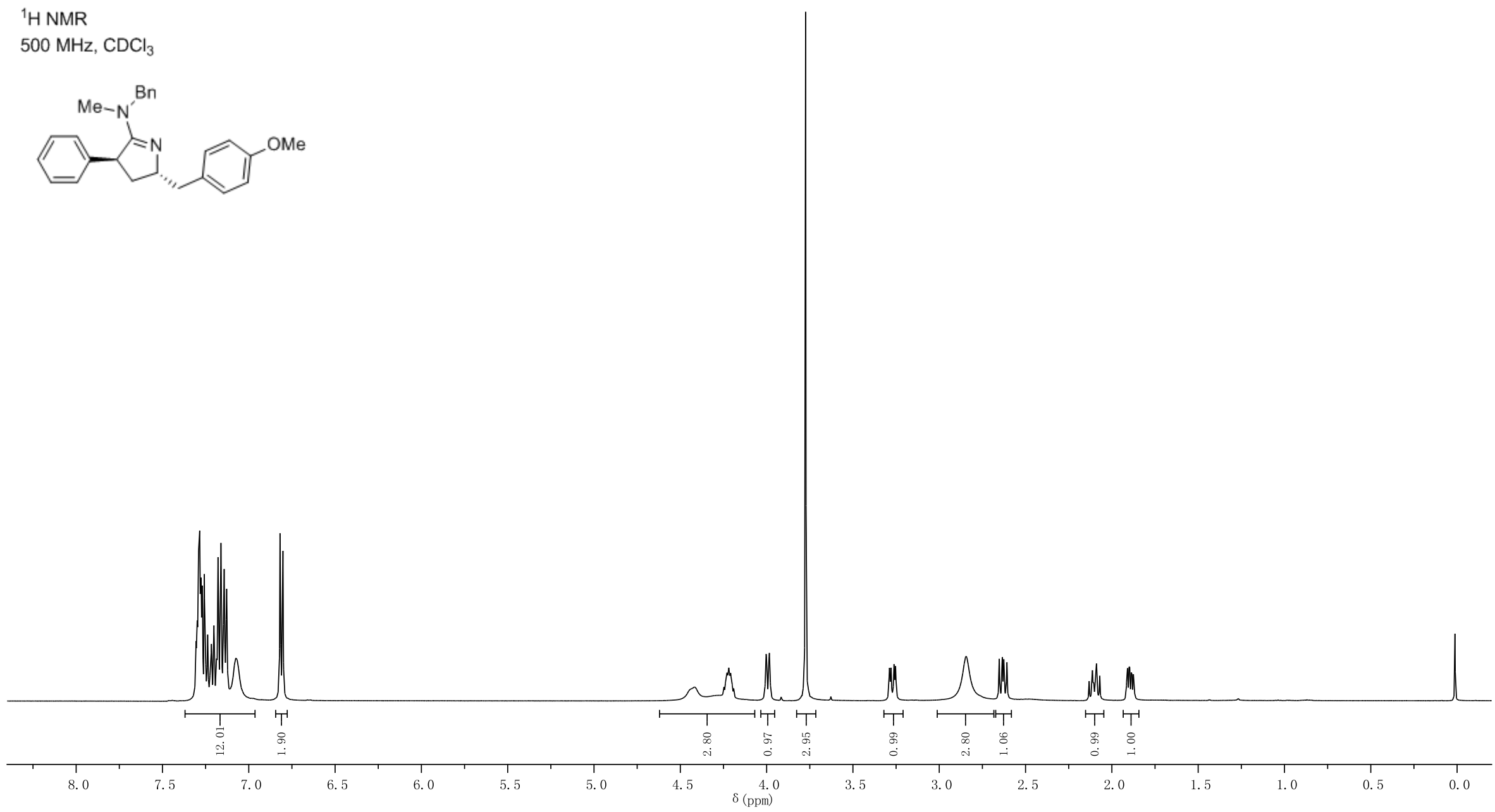


\section{Compound ( \pm )-3zaa}

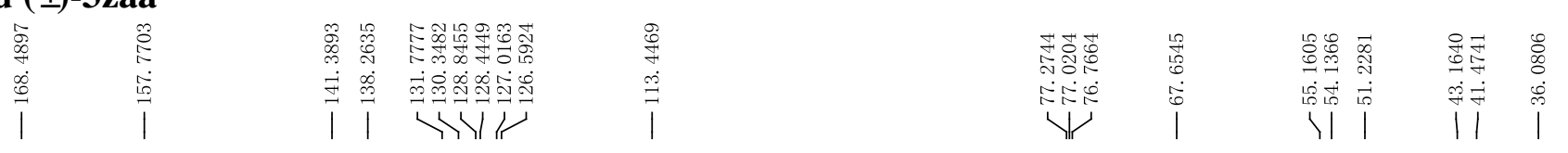

${ }^{13} \mathrm{C}\left\{{ }^{1} \mathrm{H}\right\}$ NMR

$125 \mathrm{MHz}, \mathrm{CDCl}_{3}$
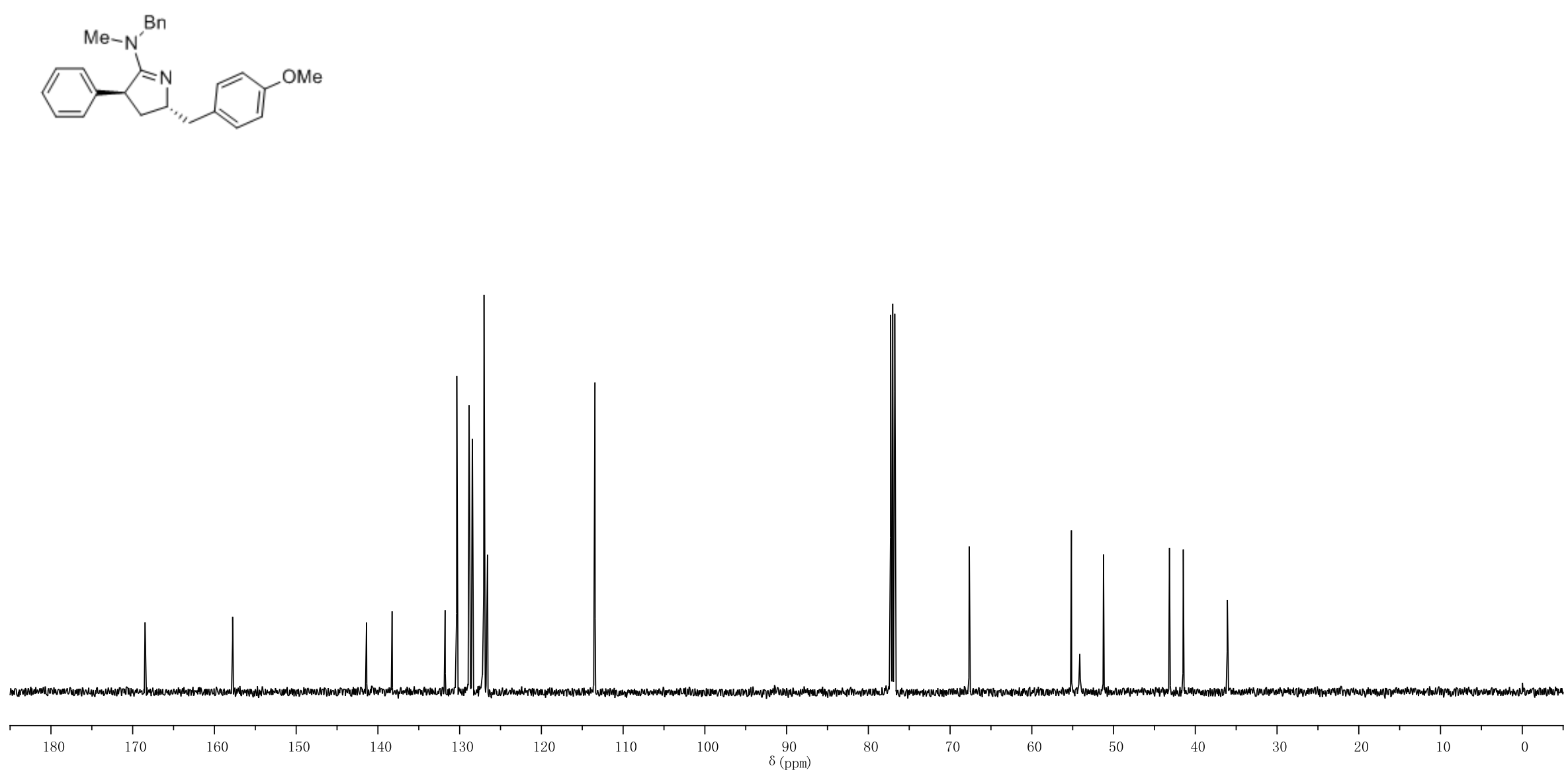


\section{Compound ( \pm )-3zba}

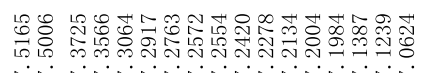

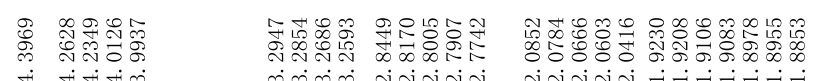

i

용융

)

${ }^{1} \mathrm{H}$ NMR

$500 \mathrm{MHz}, \mathrm{CDCl}_{3}$
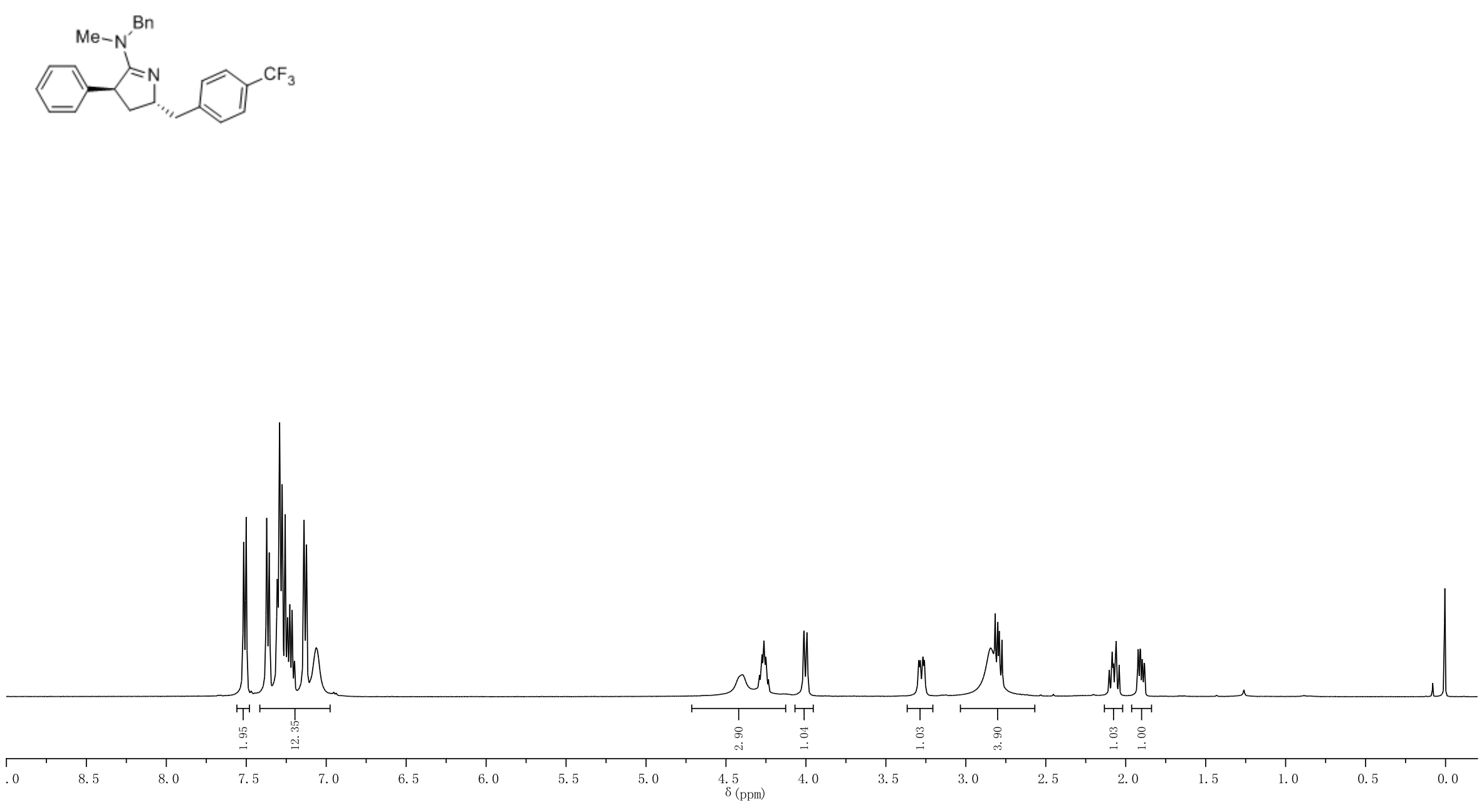
Compound ( \pm )-3zba

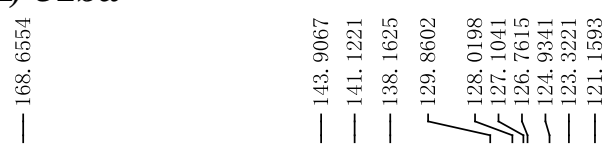

${ }^{13} \mathrm{C}\left\{{ }^{1} \mathrm{H}\right\}$ NMR

$125 \mathrm{MHz}, \mathrm{CDCl}_{3}$

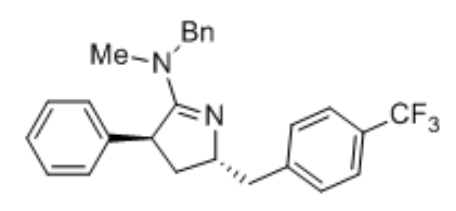

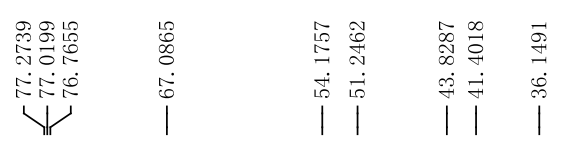

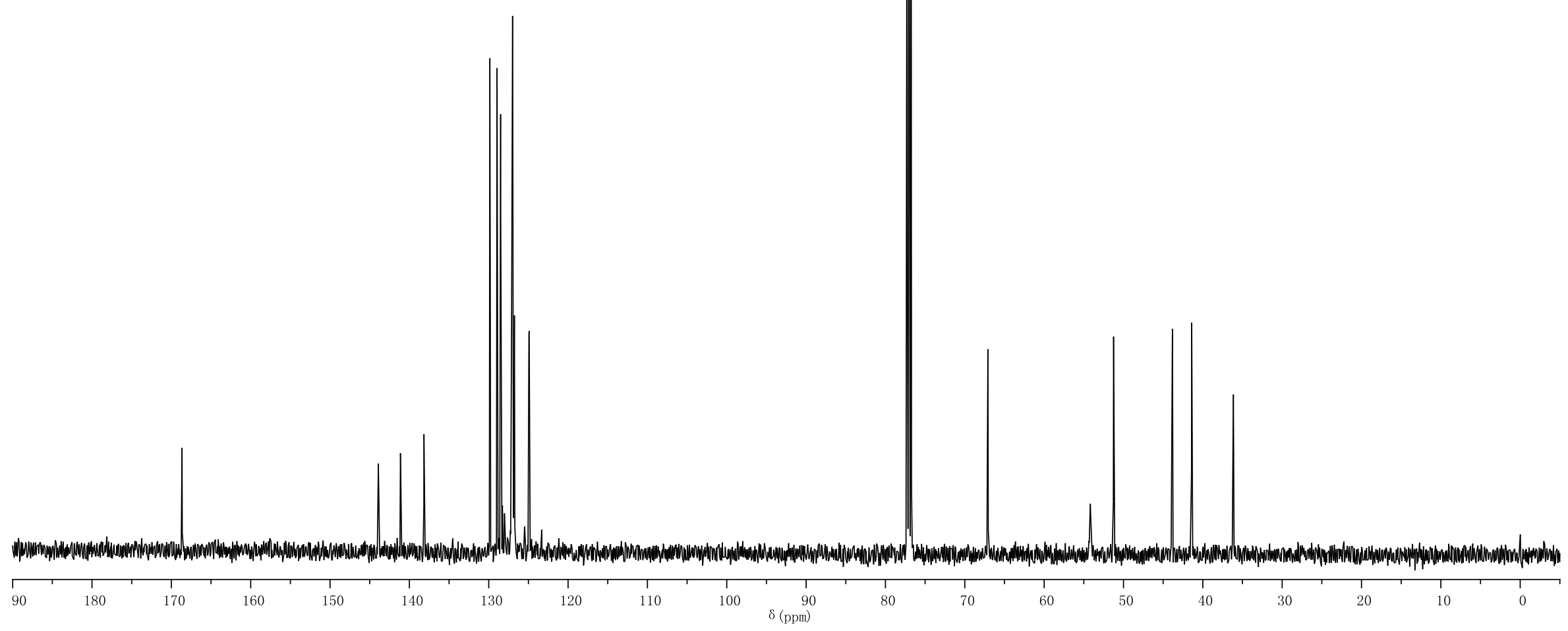




\section{Compound ( \pm )-3zca}

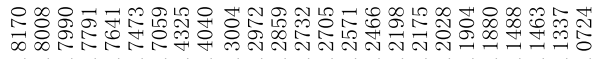

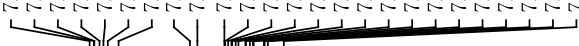

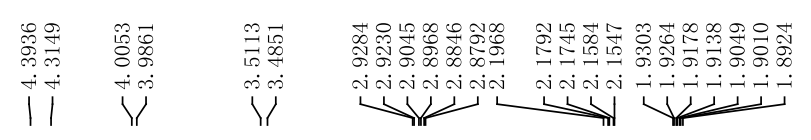

${ }^{1} \mathrm{H}$ NMR

$500 \mathrm{MHz}, \mathrm{CDCl}_{3}$
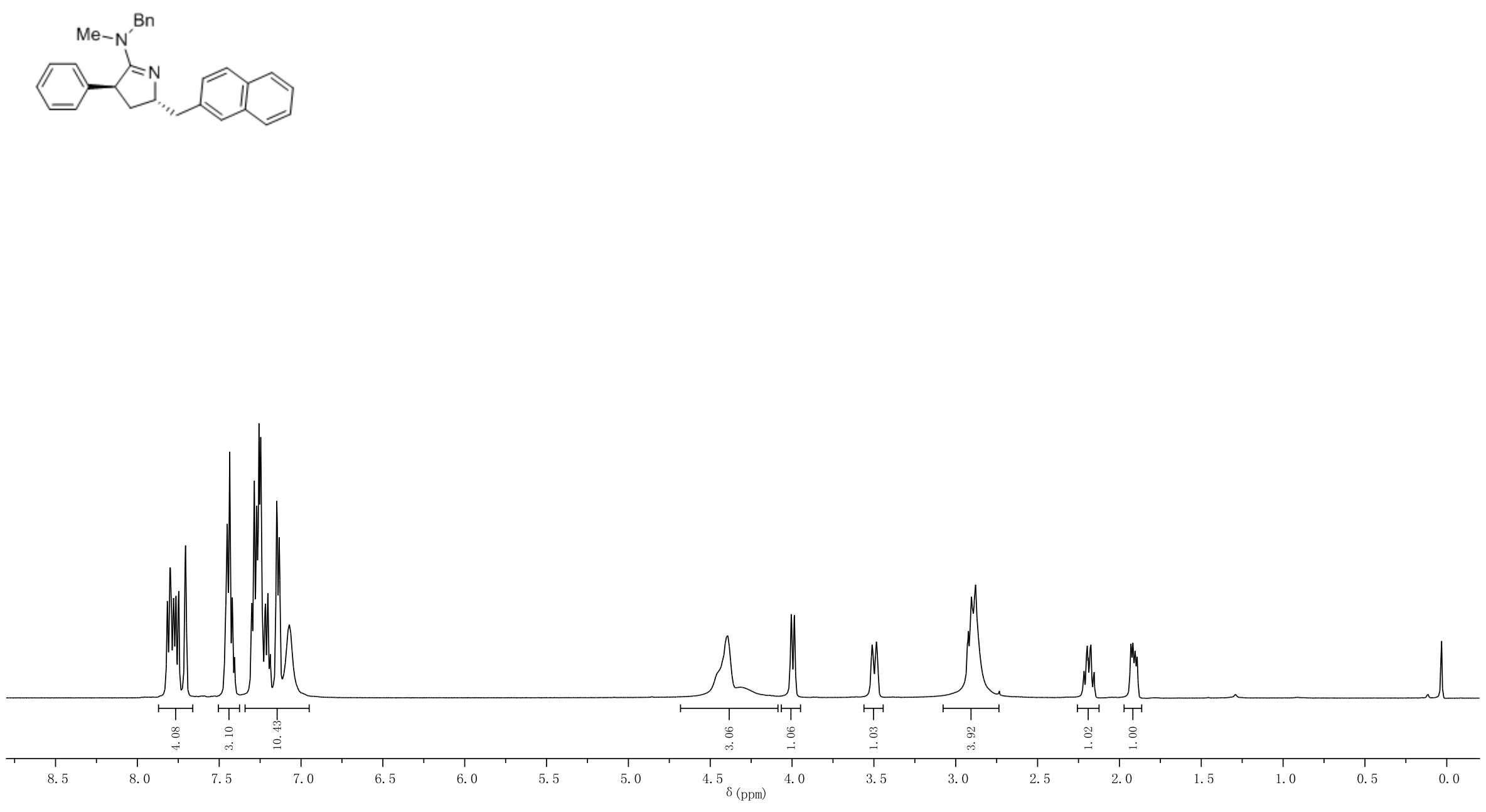


\section{Compound ( \pm )-3zca}

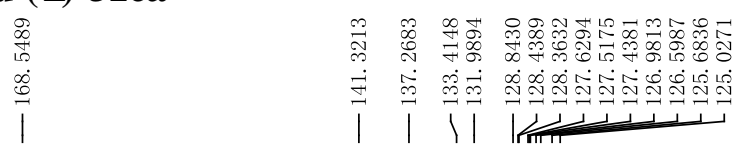

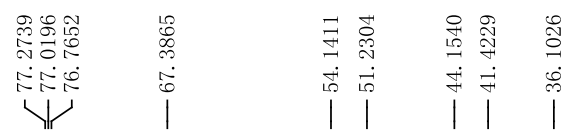

${ }^{13} \mathrm{C}\left\{{ }^{1} \mathrm{H}\right\}$ NMR

$125 \mathrm{MHz}, \mathrm{CDCl}_{3}$
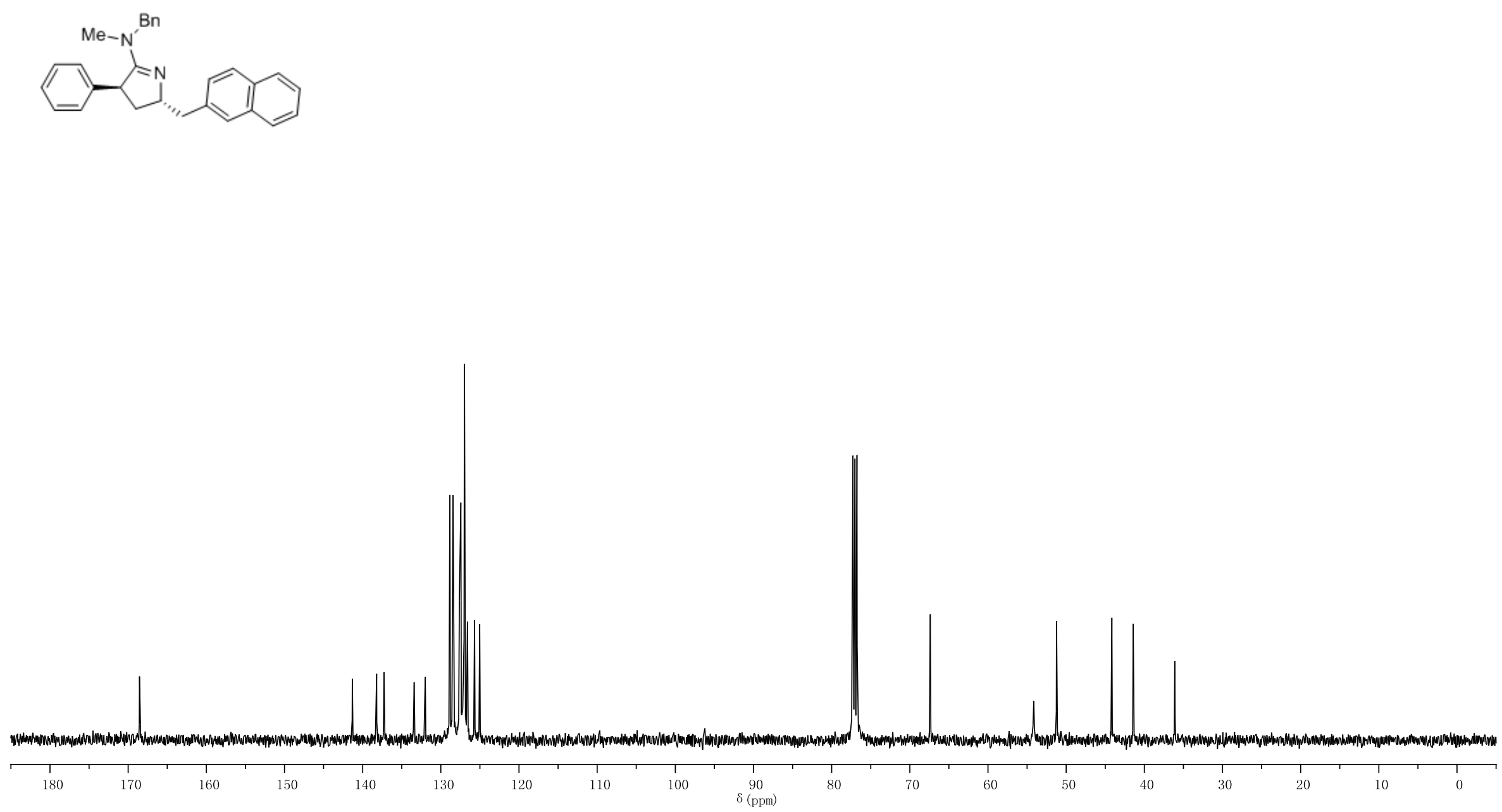


\section{Compound ( \pm )-3zda}

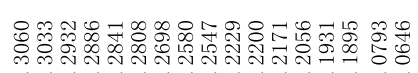

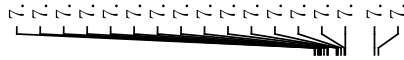

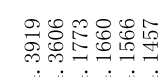

ปان

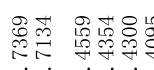

vi nivin

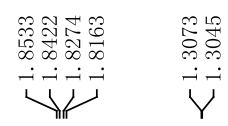

${ }^{1} \mathrm{H}$ NMR

$500 \mathrm{MHz}, \mathrm{CDCl}_{3}$
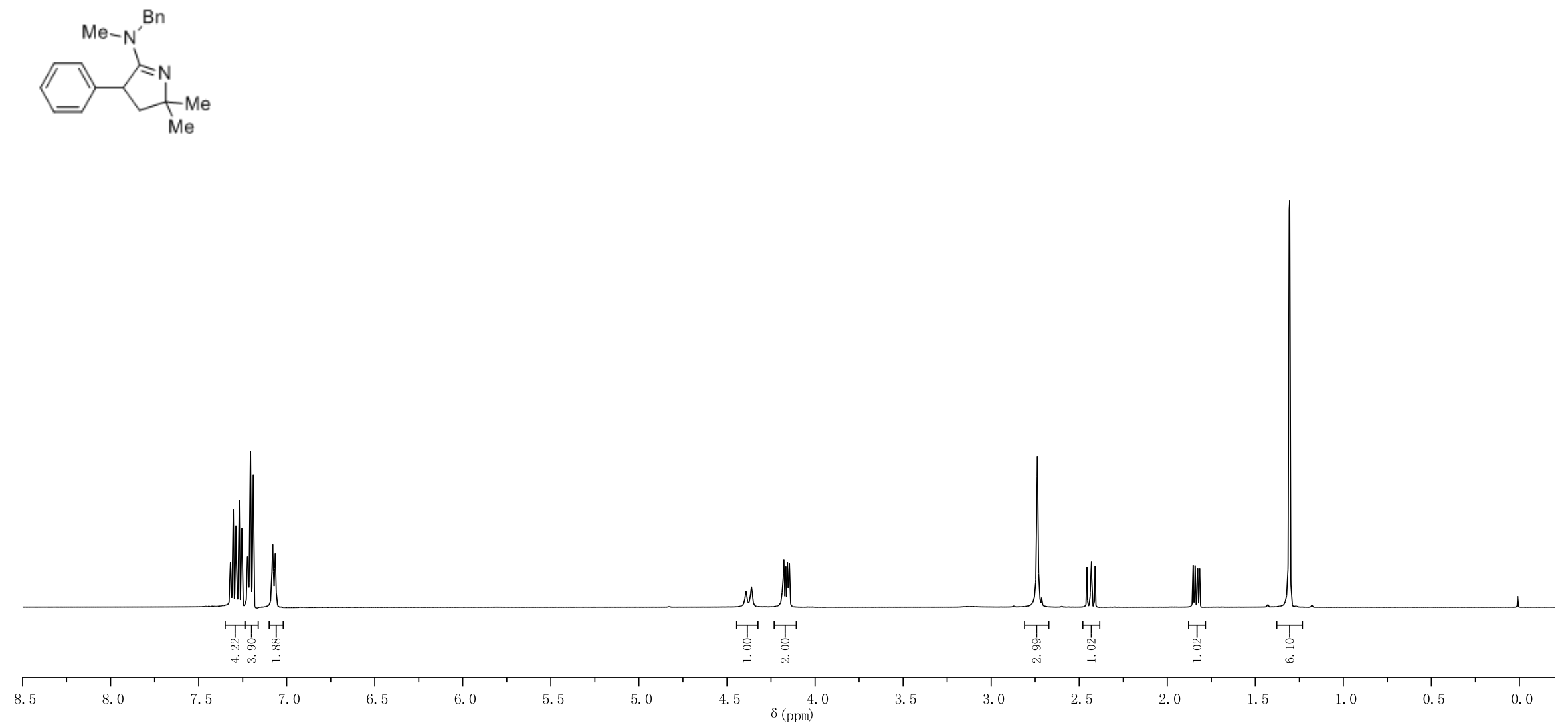


\section{Compound ( \pm )-3zda}

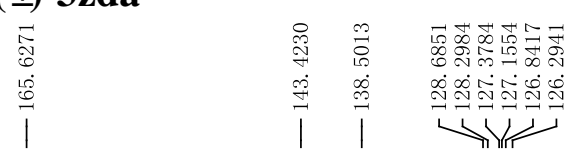

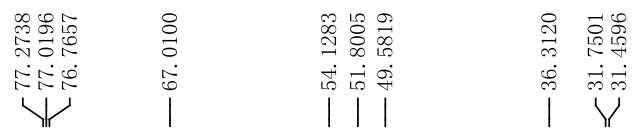

${ }^{13} \mathrm{C}\left\{{ }^{1} \mathrm{H}\right\}$ NMR

$125 \mathrm{MHz}, \mathrm{CDCl}_{3}$
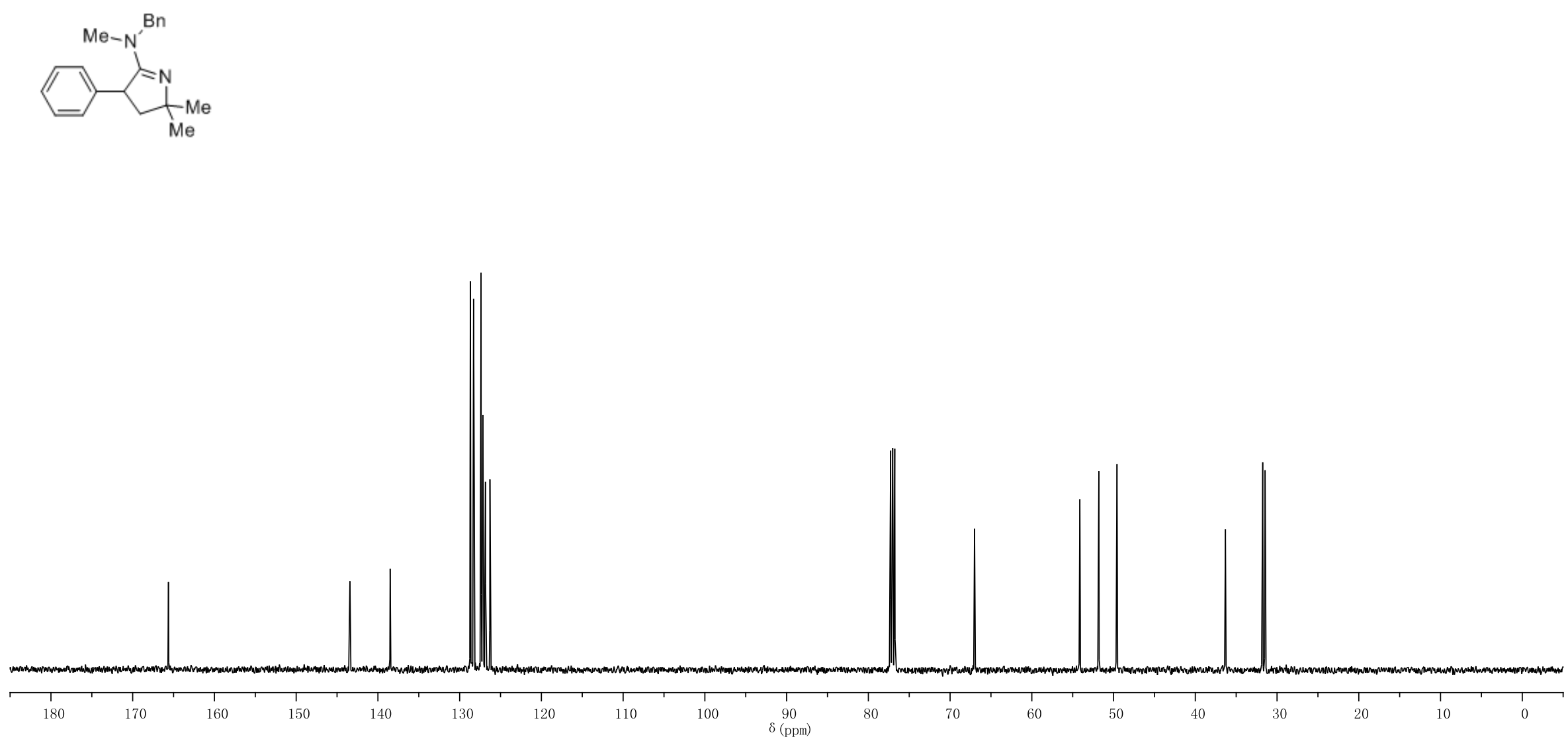


\section{Compound ( \pm )-3zfa}

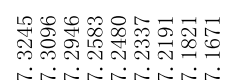

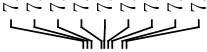

${ }^{1} \mathrm{H}$ NMR

$500 \mathrm{MHz}, \mathrm{CDCl}_{3}$
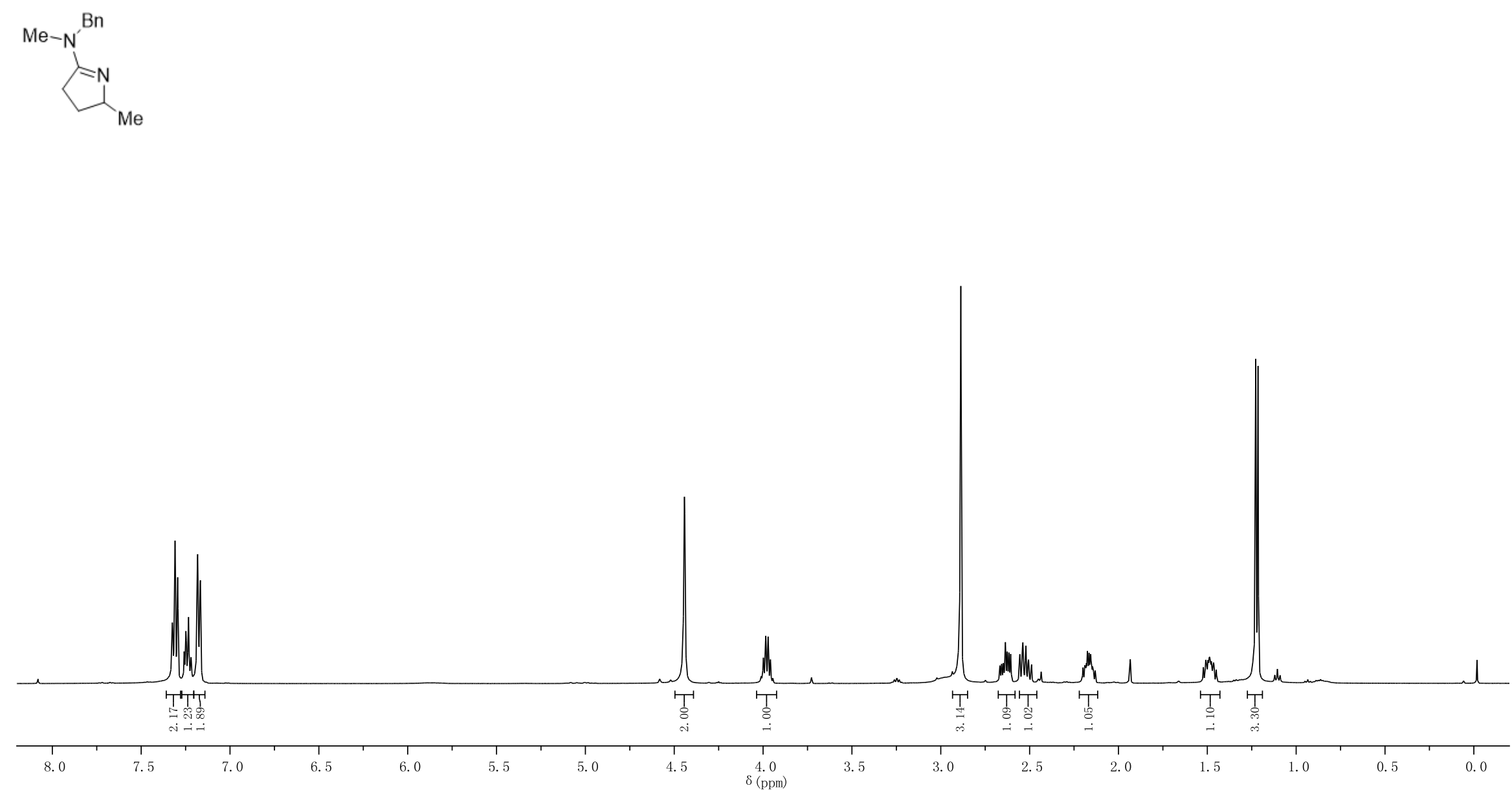


\section{Compound ( \pm )-3zfa}

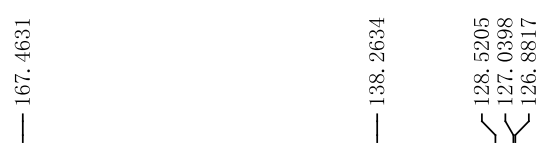

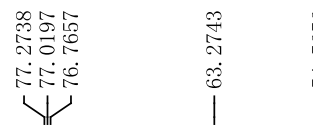

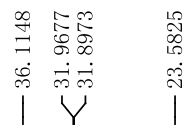

${ }^{13} \mathrm{C}\left\{{ }^{1} \mathrm{H}\right\}$ NMR

$125 \mathrm{MHz}, \mathrm{CDCl}_{3}$
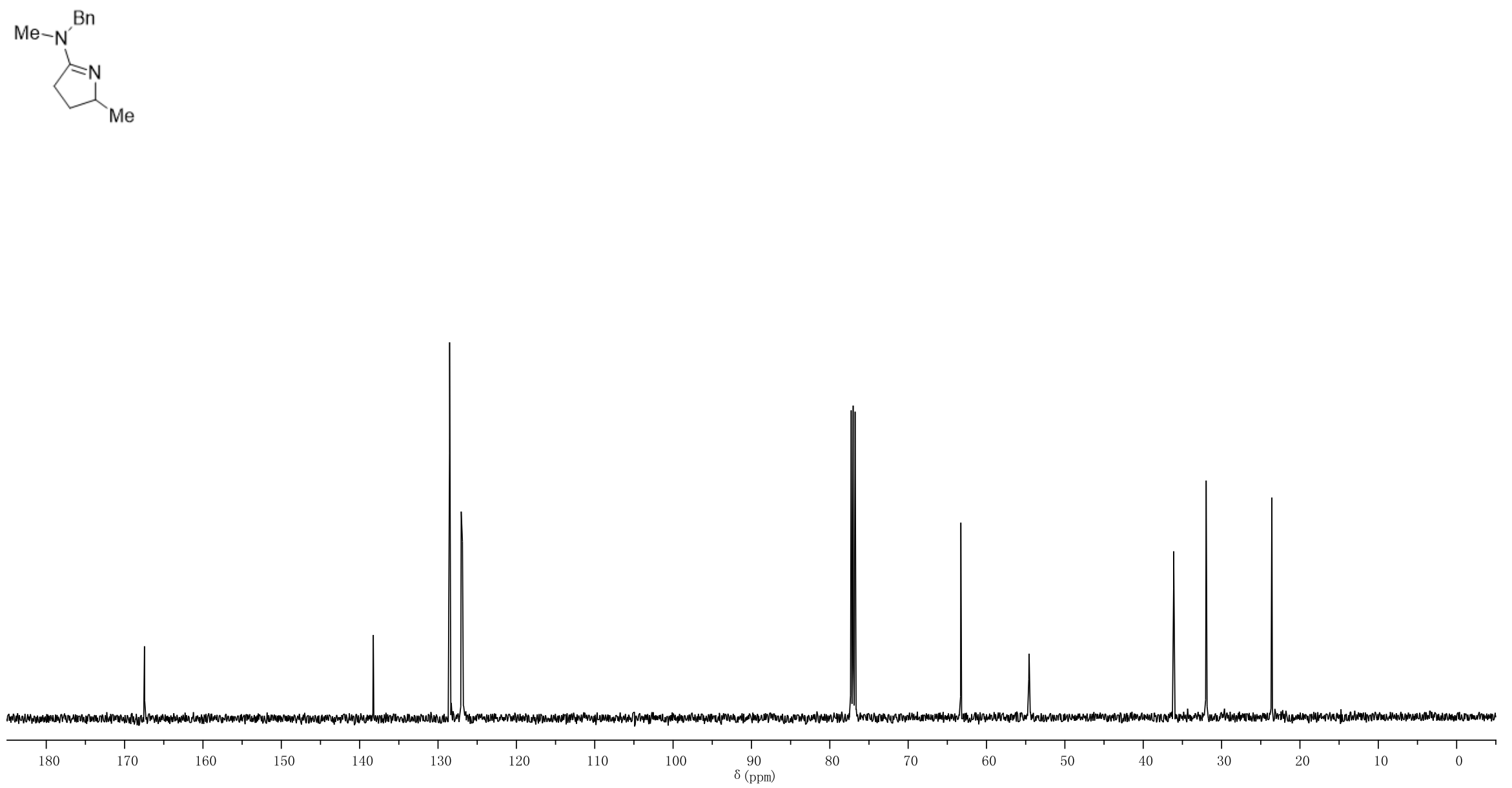


\section{Compound ( \pm )-3zia}

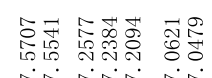

irivi

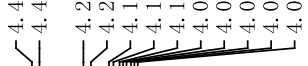

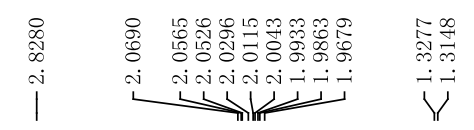

${ }^{1} \mathrm{H}$ NMR

$500 \mathrm{MHz}, \mathrm{CDCl}_{3}$
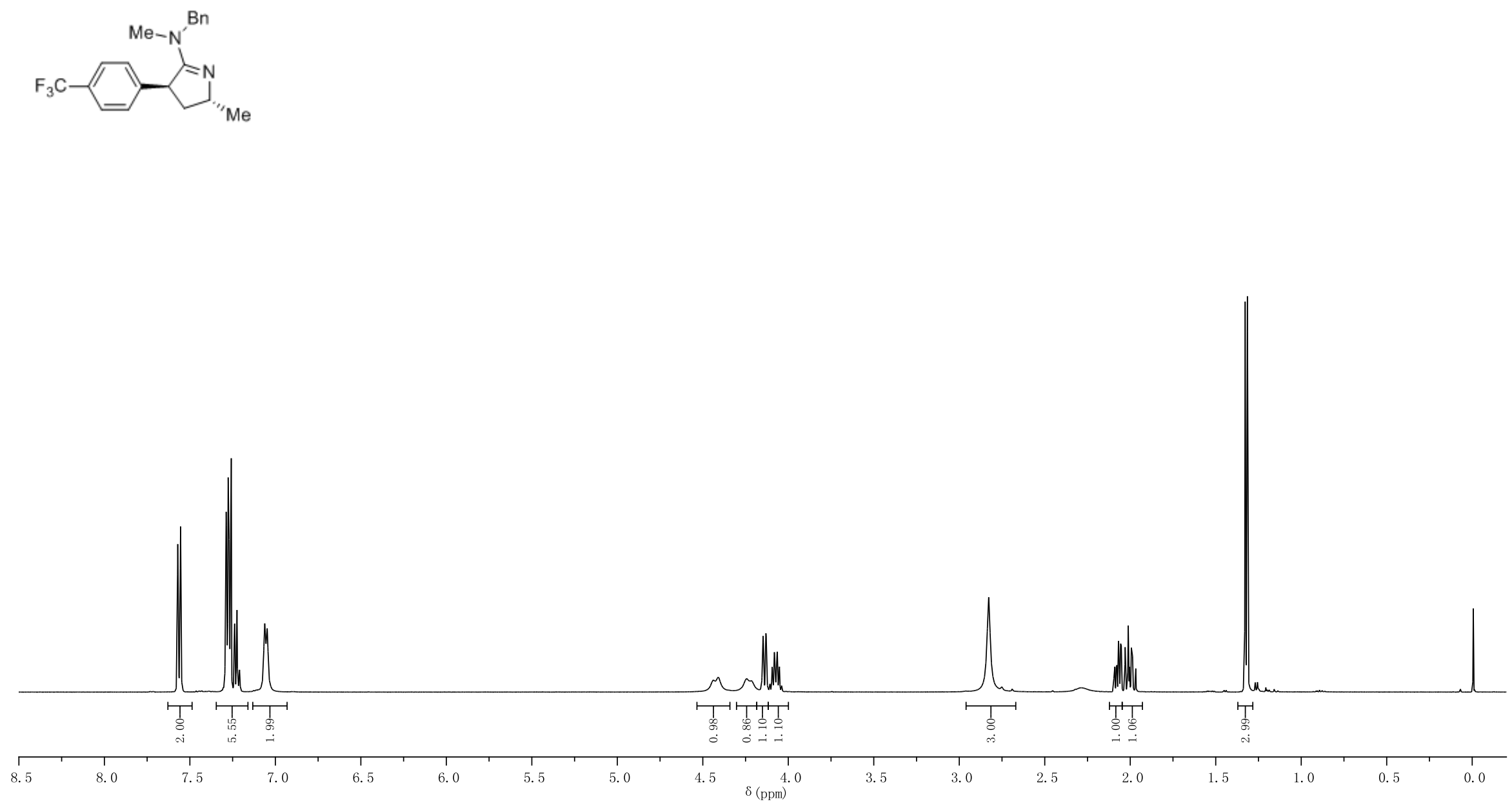


\section{Compound ( \pm )-3zia}

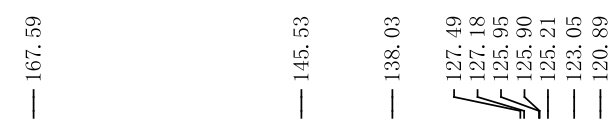

${ }^{13} \mathrm{C}\left\{{ }^{1} \mathrm{H}\right\}$ NMR

$125 \mathrm{MHz} \mathrm{CDCl}_{3}$
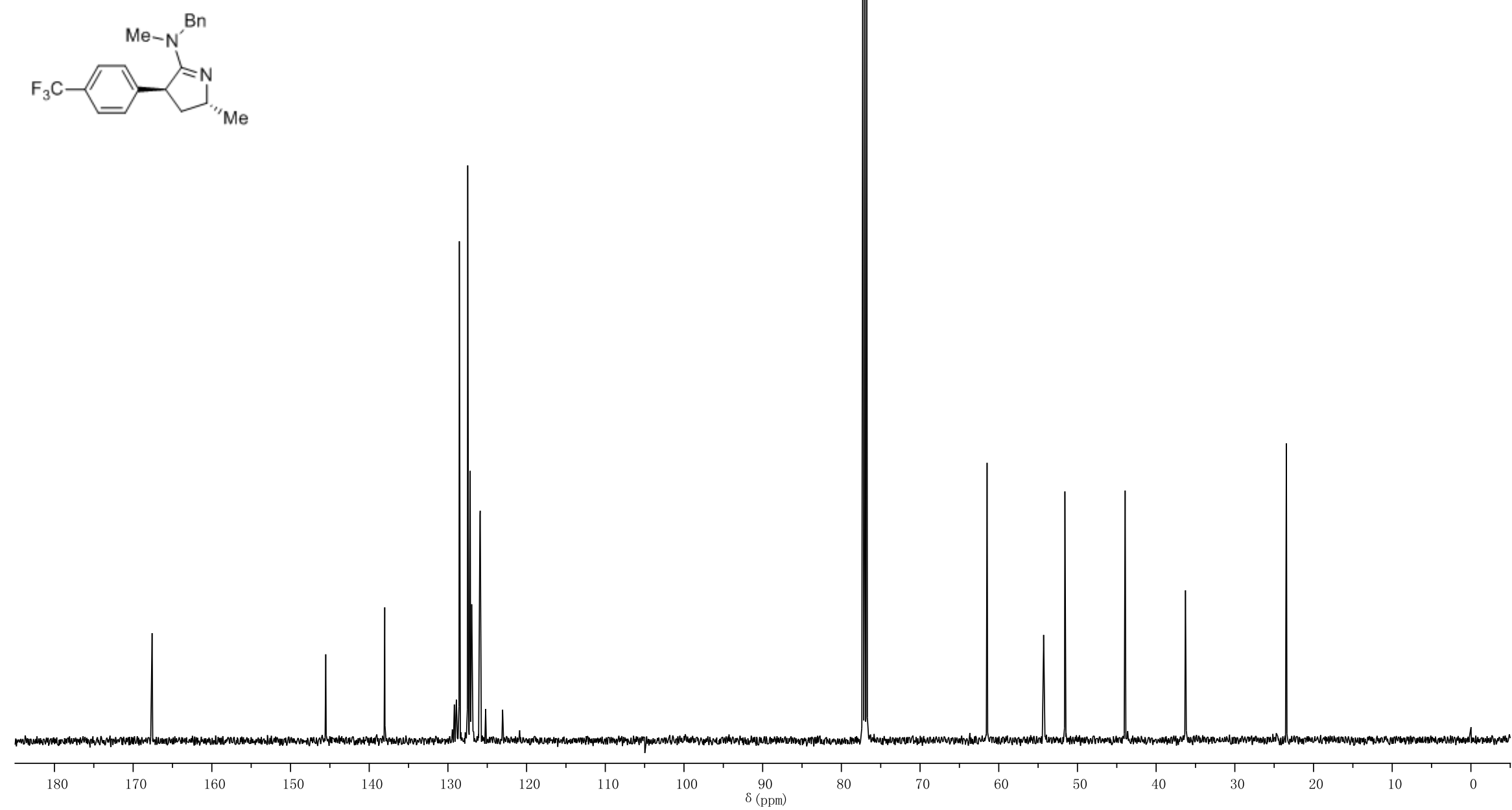


\section{Compound ( \pm )-3zja}

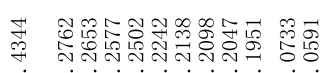

jenininivin

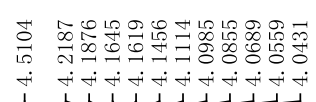

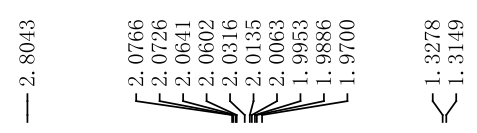

${ }^{1} \mathrm{H}$ NMR

$500 \mathrm{MHz}, \mathrm{CDCl}_{3}$
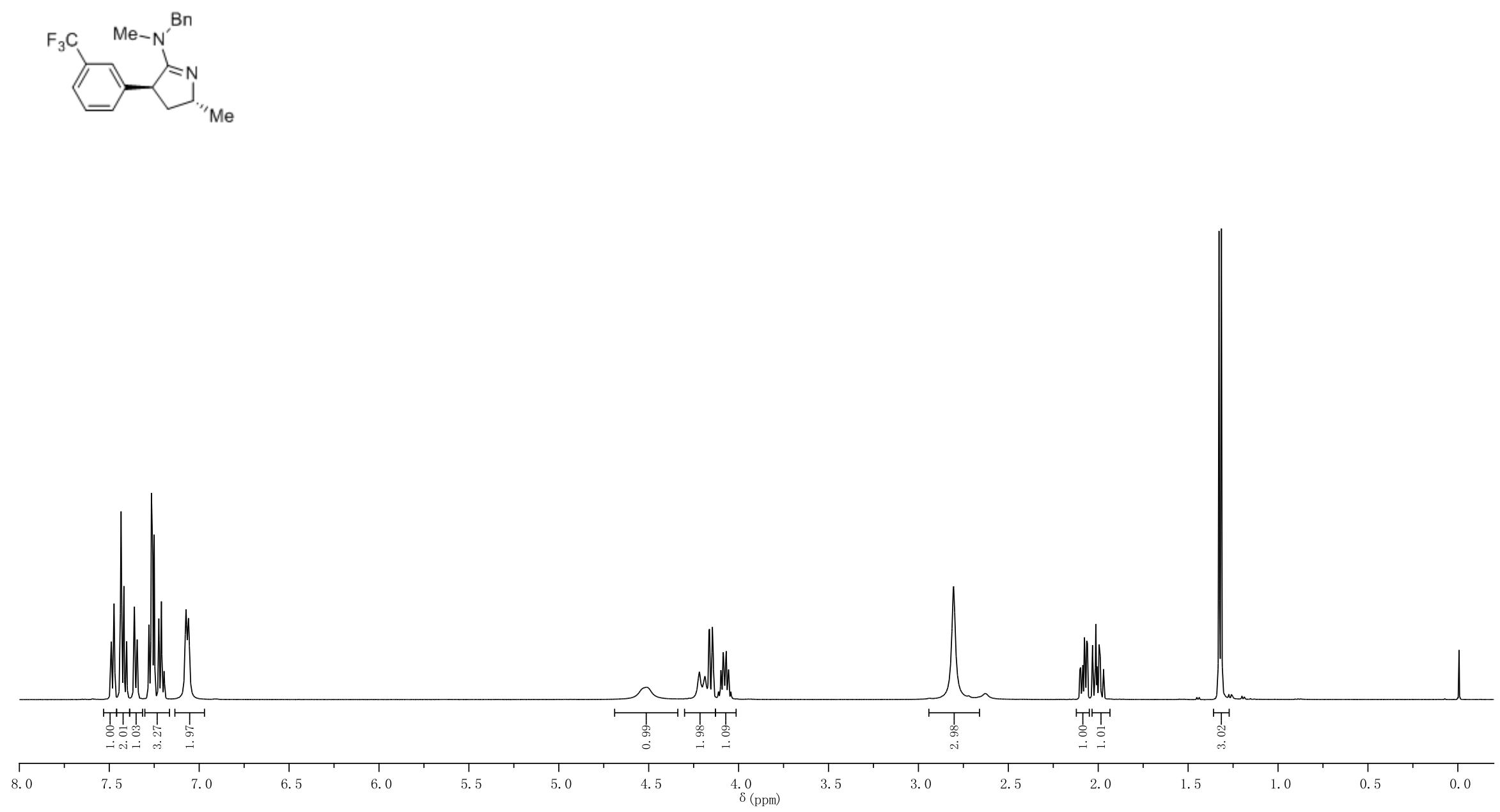


\section{Compound ( \pm )-3zja}

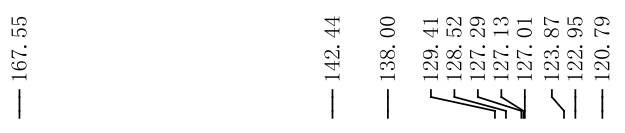

${ }^{13} \mathrm{C}\left\{{ }^{1} \mathrm{H}\right\}$ NMR

$125 \mathrm{MHz}, \mathrm{CDCl}_{3}$

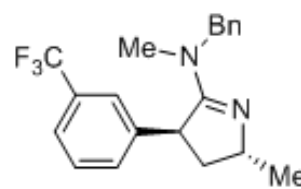

Me

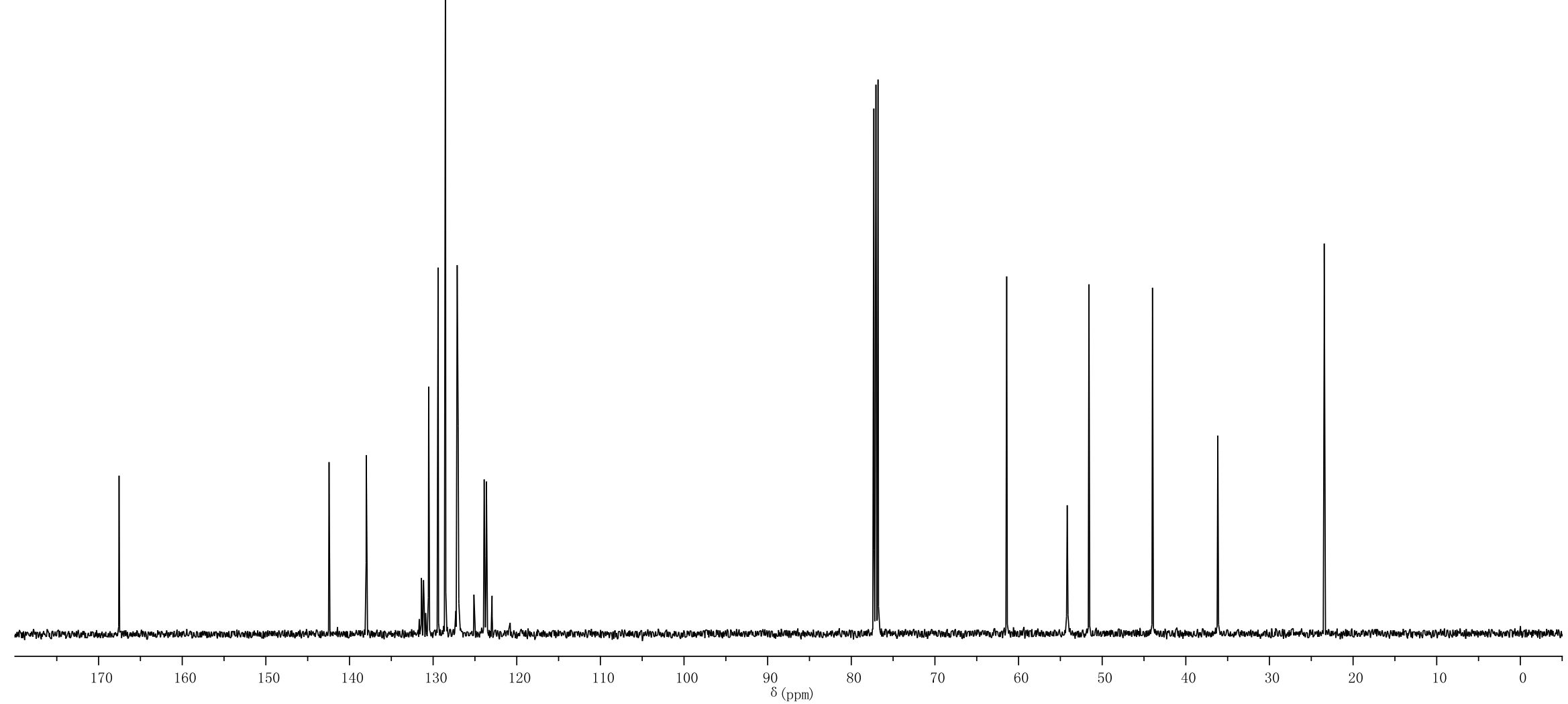




\section{Compound ( \pm )-3zka (containing diastereomer)}

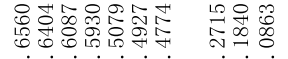

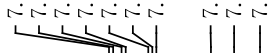

${ }^{1} \mathrm{H}$ NMR

$500 \mathrm{MHz}, \mathrm{CDCl}_{3}$

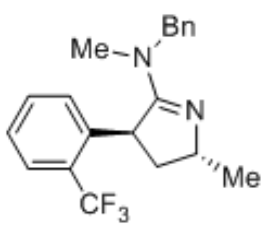

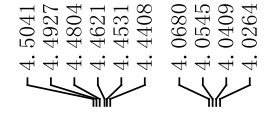

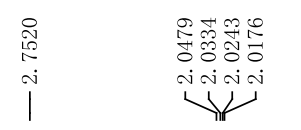

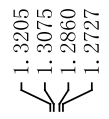

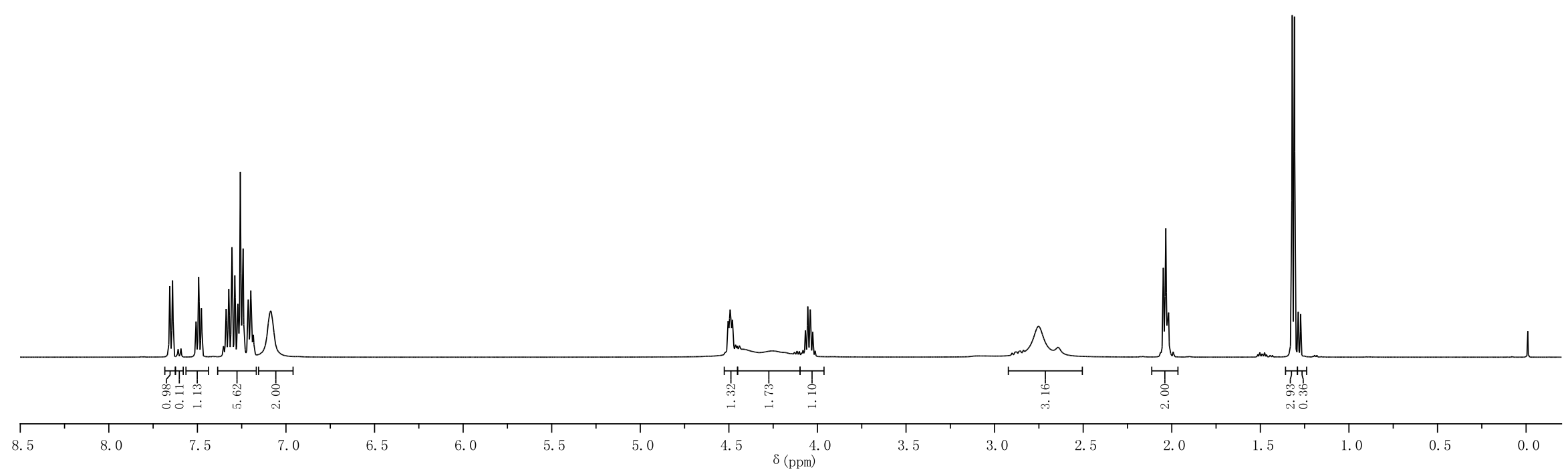




\section{Compound ( \pm )-3zka (containing diastereomer)}

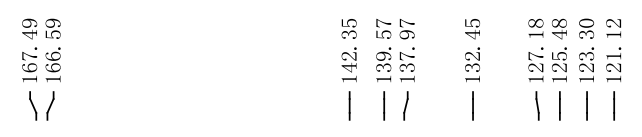

${ }^{13} \mathrm{C}\left\{{ }^{1} \mathrm{H}\right\}$ NMR

$125 \mathrm{MHz}, \mathrm{CDCl}_{3}$
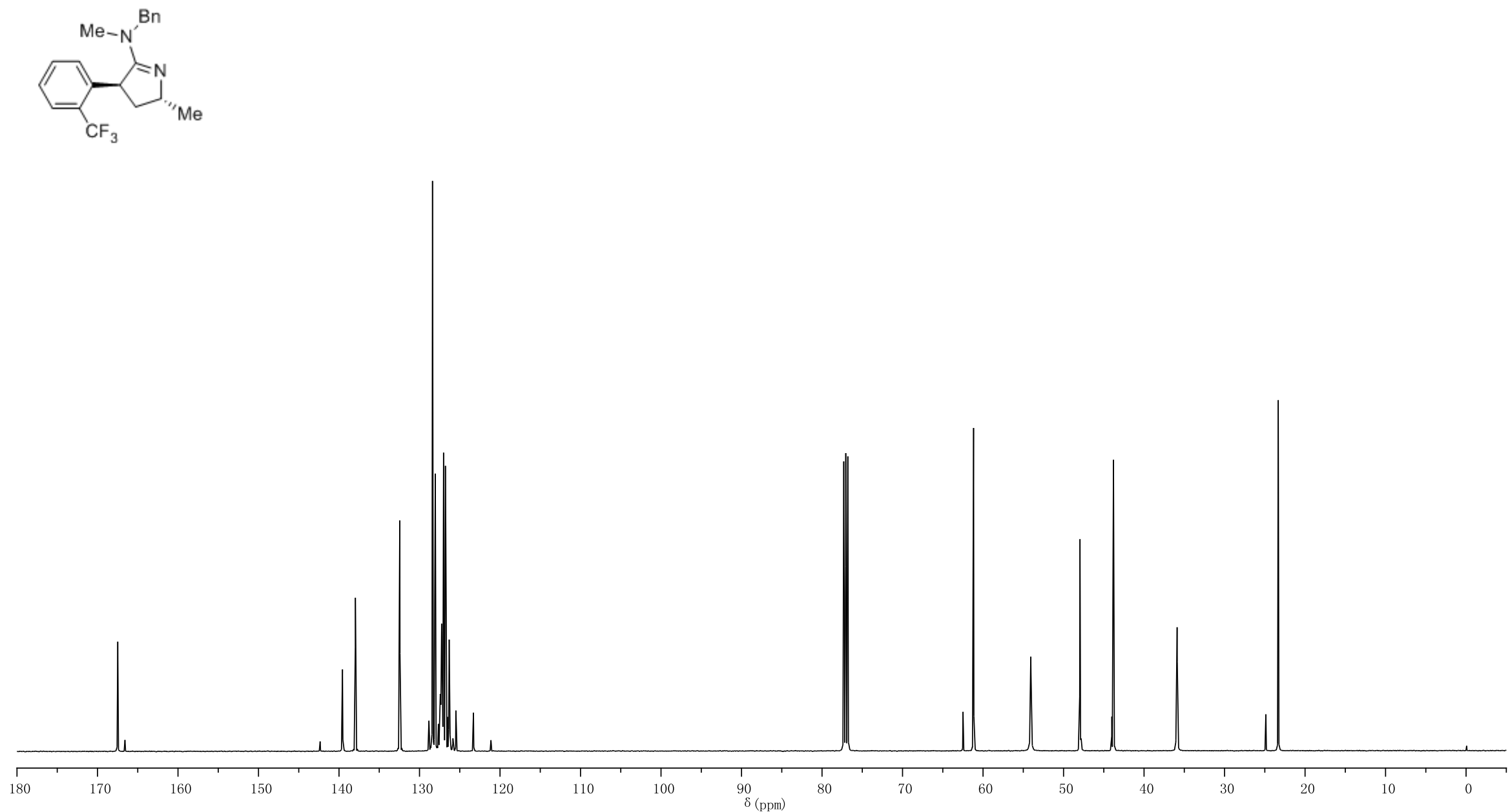


\section{Compound ( \pm )-3ab}

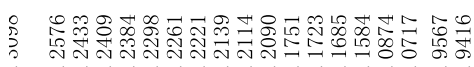

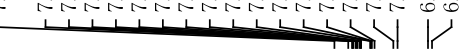

$500 \mathrm{MHz}, \mathrm{CDCl}_{3}$
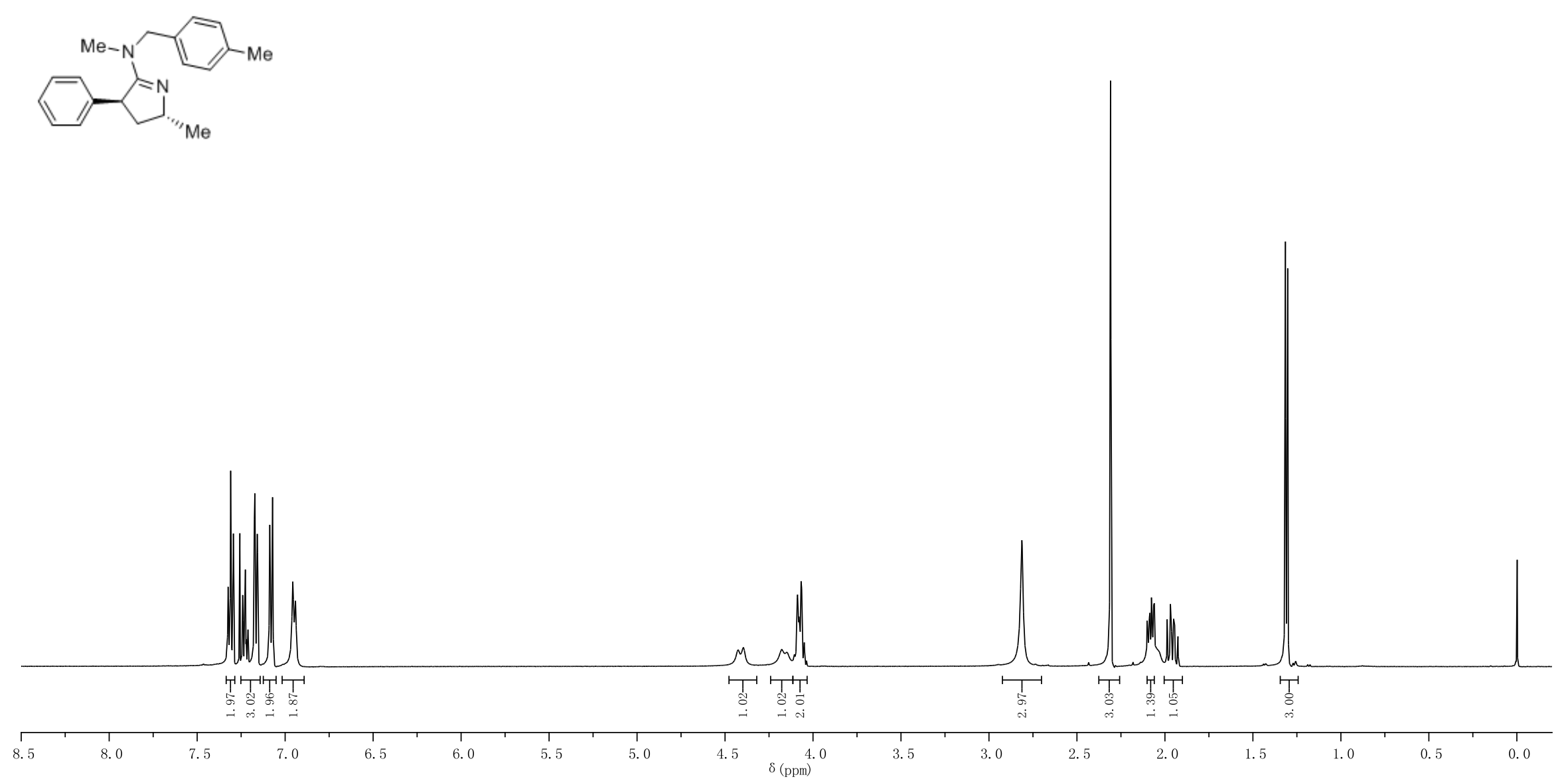
Compound ( \pm )-3ab

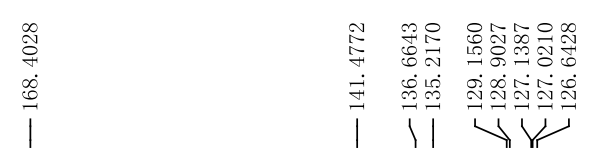

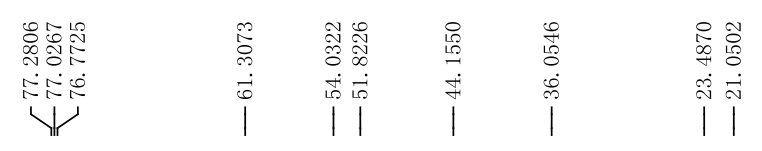

${ }^{13} \mathrm{C}\left\{{ }^{1} \mathrm{H}\right\}$ NMR

$125 \mathrm{MHz}, \mathrm{CDCl}_{3}$

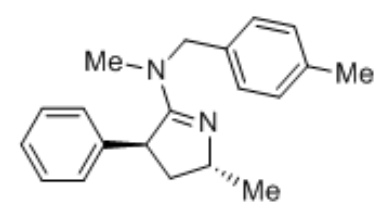

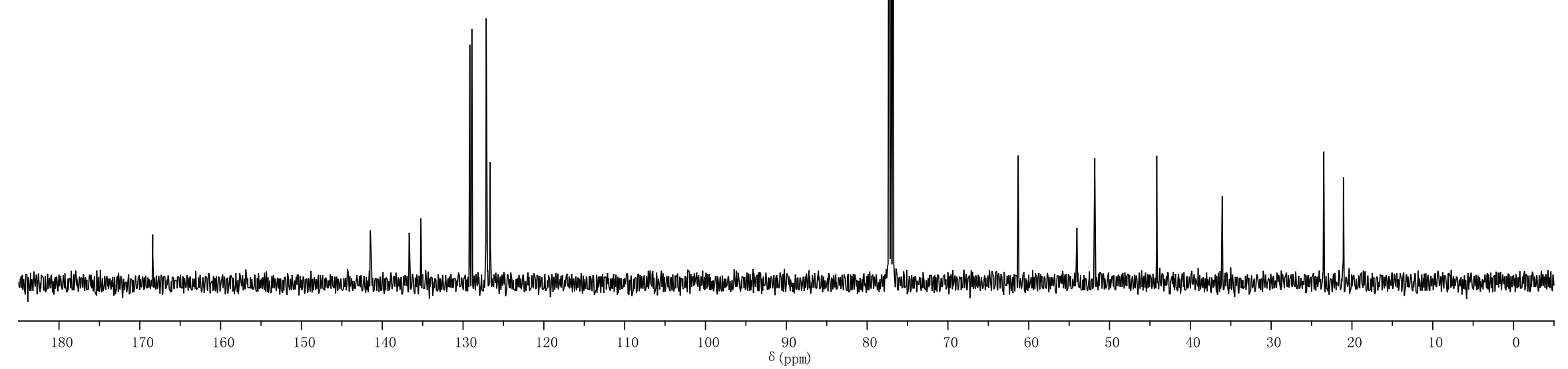




\section{Compound ( \pm )-3ac}

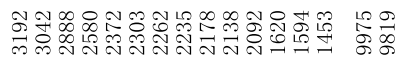

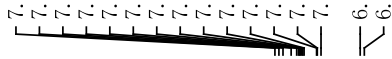

${ }^{1} \mathrm{H}$ NMR

$500 \mathrm{MHz}, \mathrm{CDCl}_{3}$
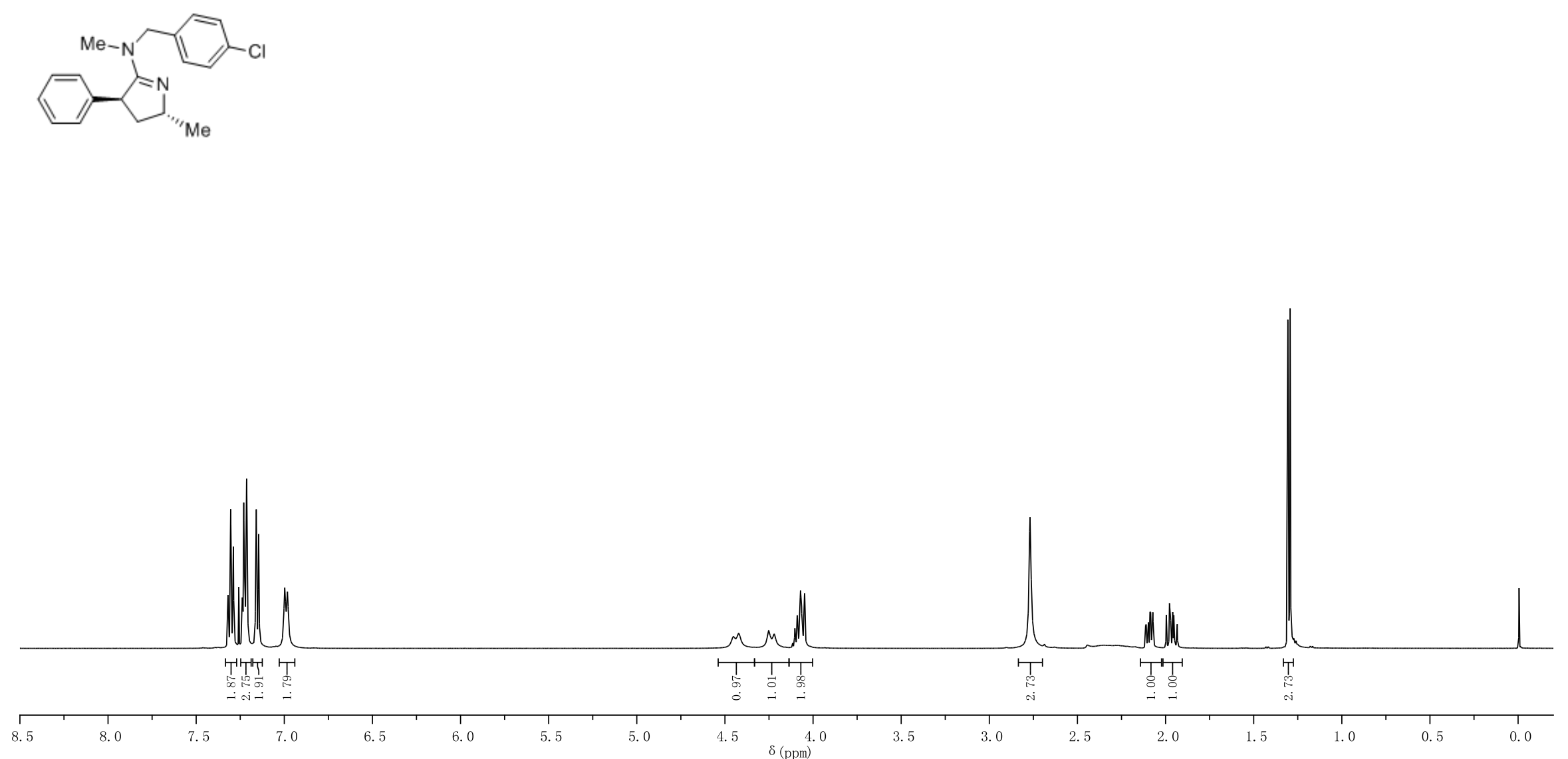


\section{Compound ( \pm )-3ac}

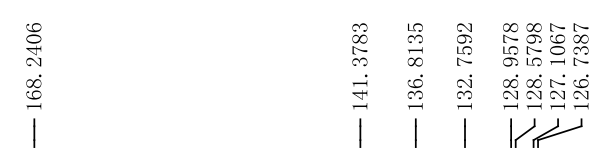

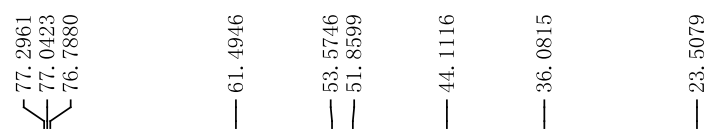

${ }^{13} \mathrm{C}\left\{{ }^{1} \mathrm{H}\right\}$ NMR

$125 \mathrm{MHz}, \mathrm{CDCl}_{3}$
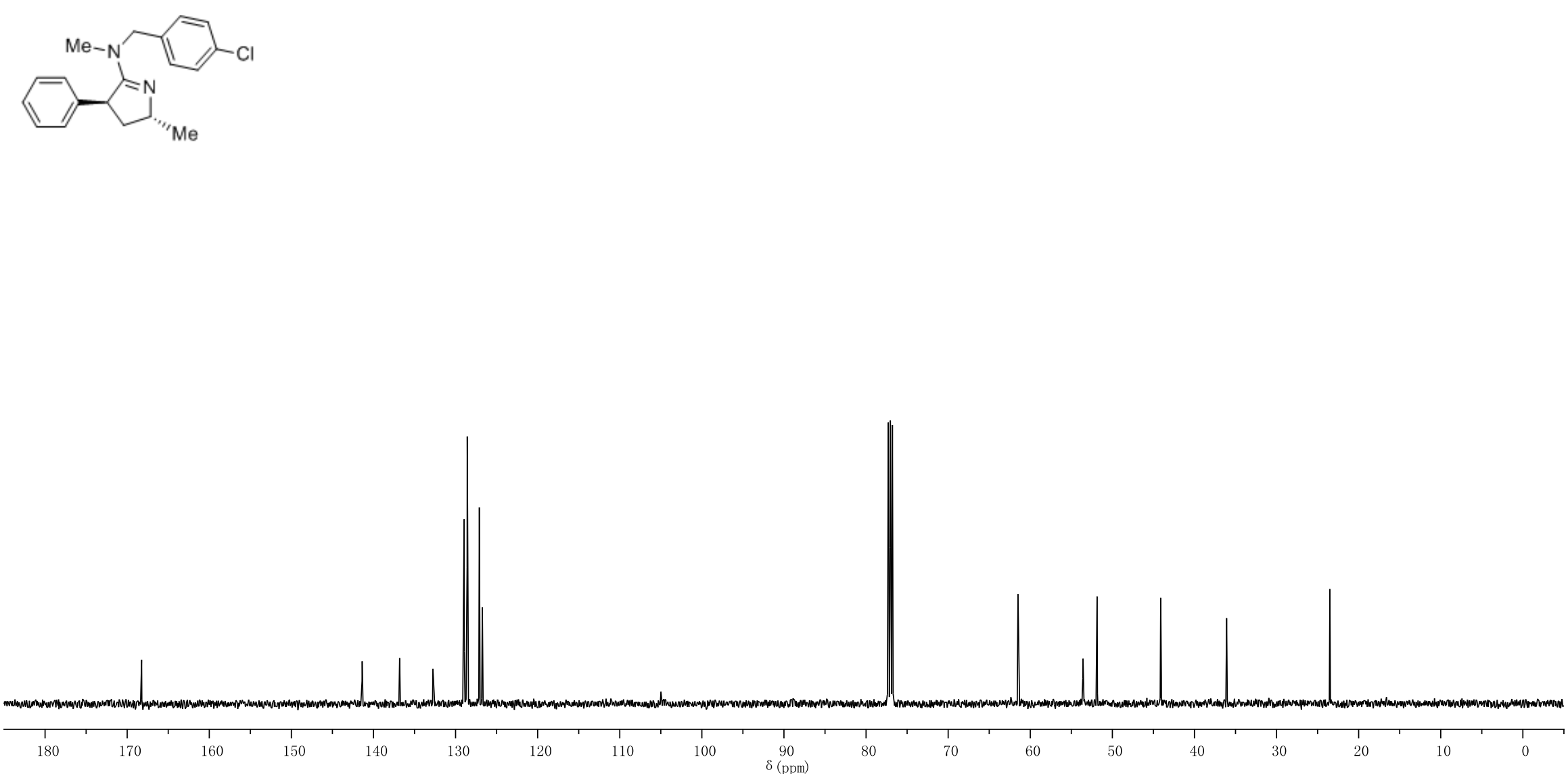


\section{Compound ( \pm )-3ad}

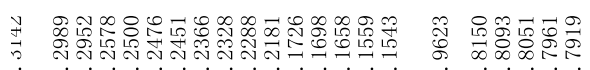

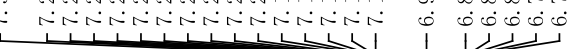

${ }^{1} \mathrm{H}$ NMR

$500 \mathrm{MHz}, \mathrm{CDCl}_{3}$
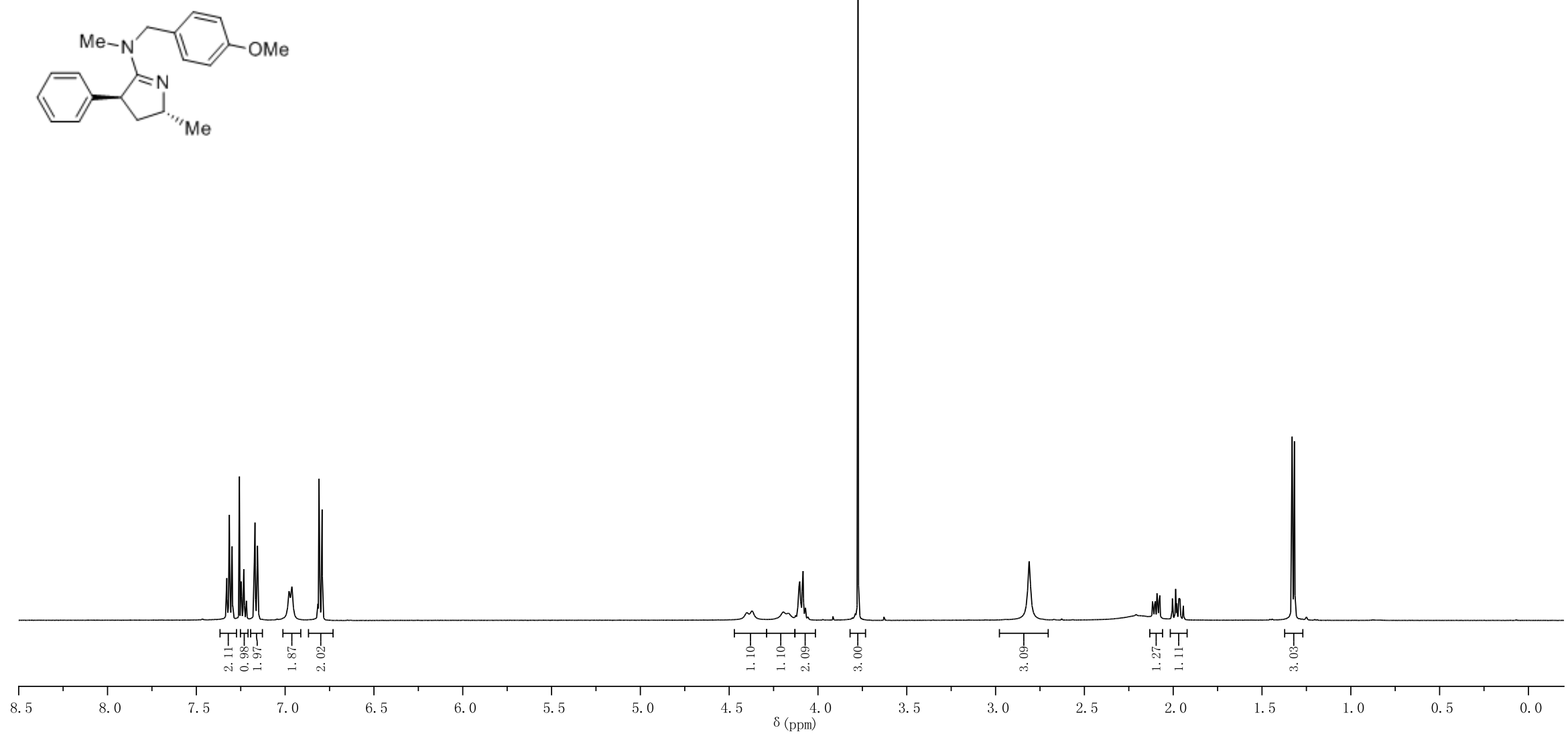


\section{Compound ( \pm )-3ad}

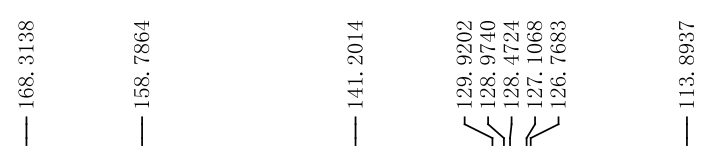

${ }^{13} \mathrm{C}\left\{{ }^{1} \mathrm{H}\right\}$ NMR

$125 \mathrm{MHz}, \mathrm{CDCl}_{3}$
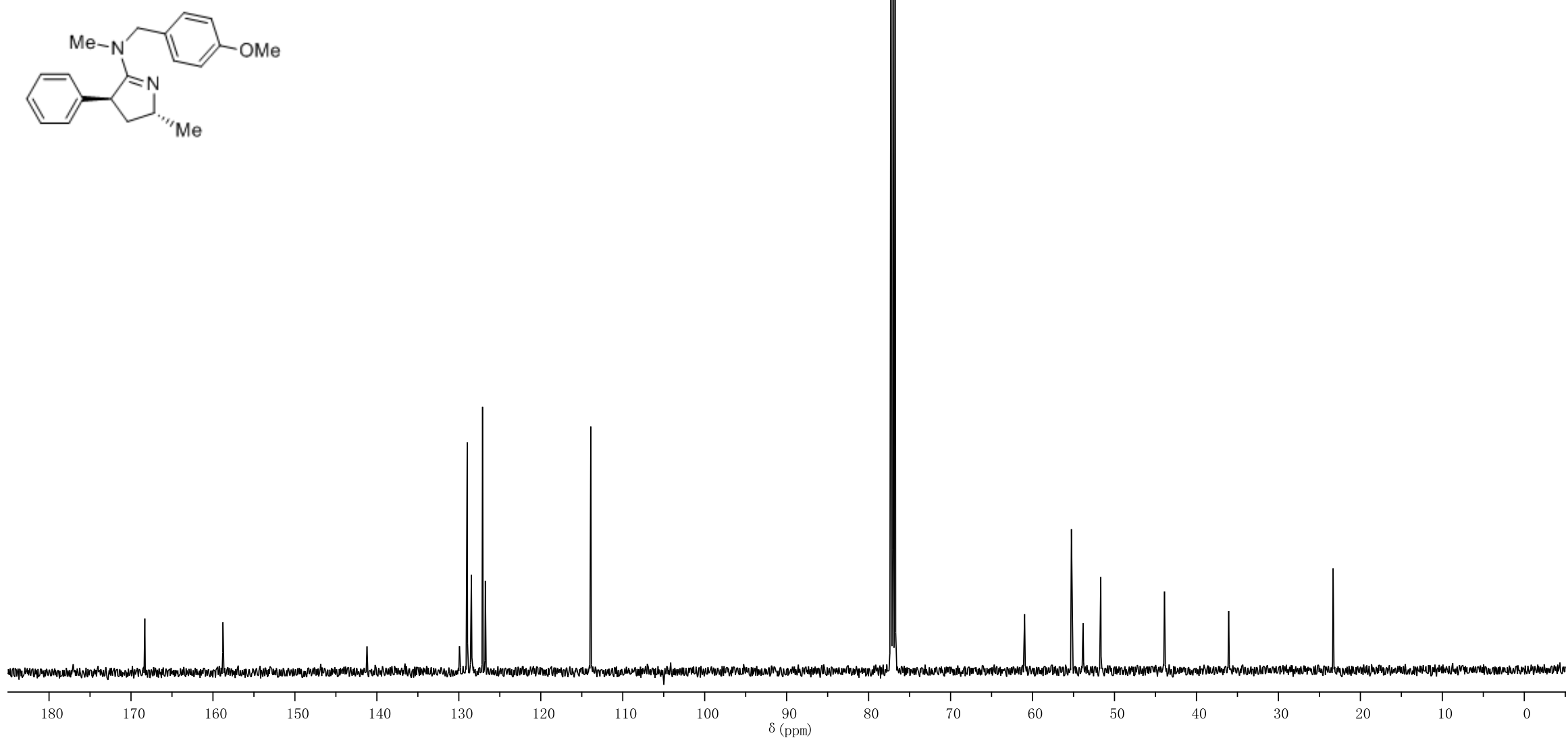


\section{Compound ( \pm )-3ae}

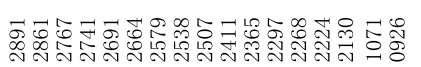

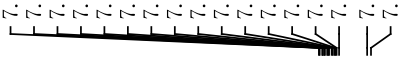

${ }^{1} \mathrm{H}$ NMR

$500 \mathrm{MHz}, \mathrm{CDCl}_{3}$
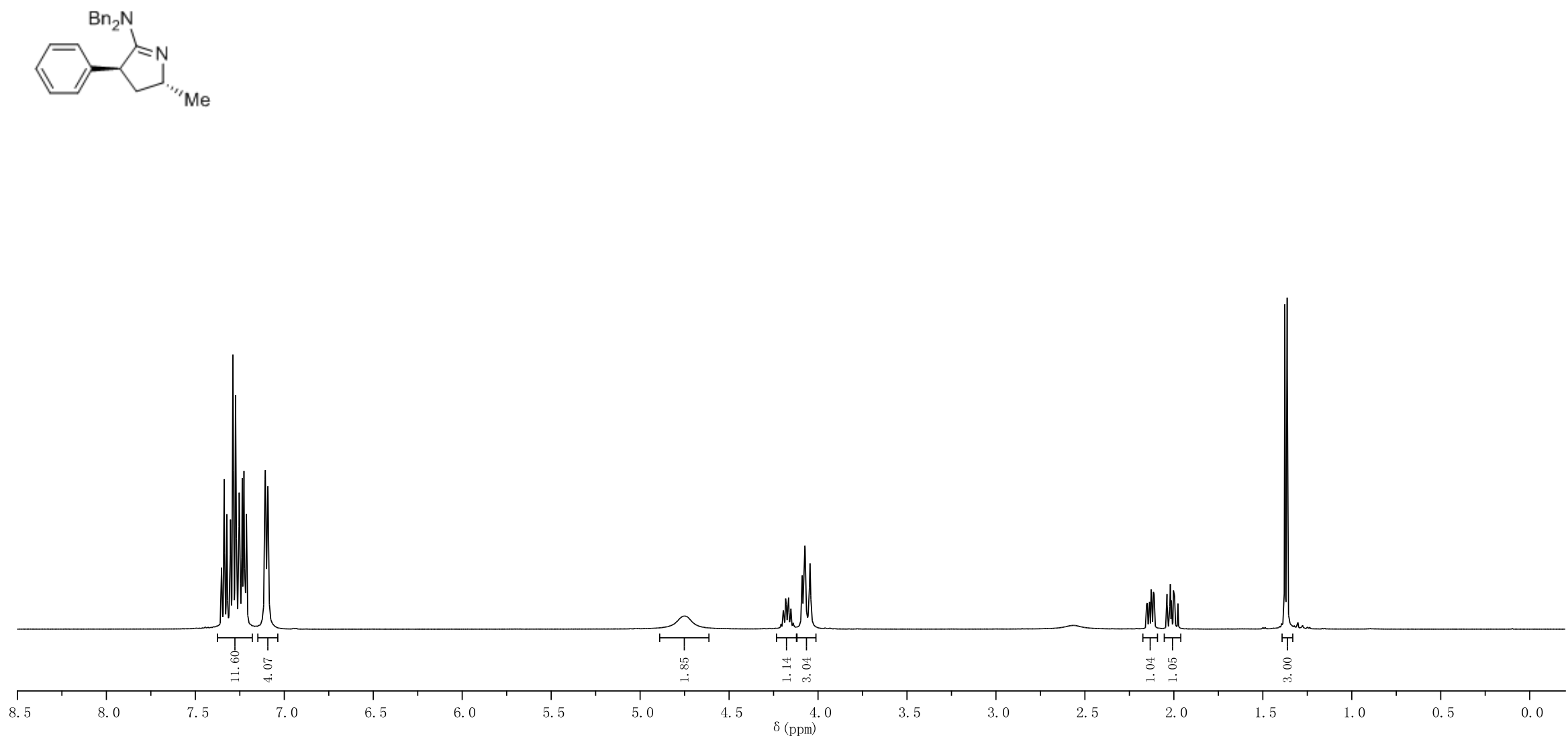
Compound ( \pm )-3ae

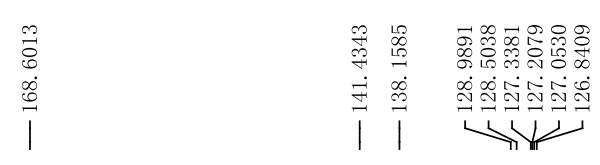

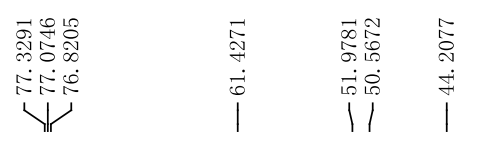

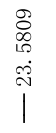

${ }^{13} \mathrm{C}\left\{{ }^{1} \mathrm{H}\right\}$ NMR

$125 \mathrm{MHz}, \mathrm{CDCl}_{3}$
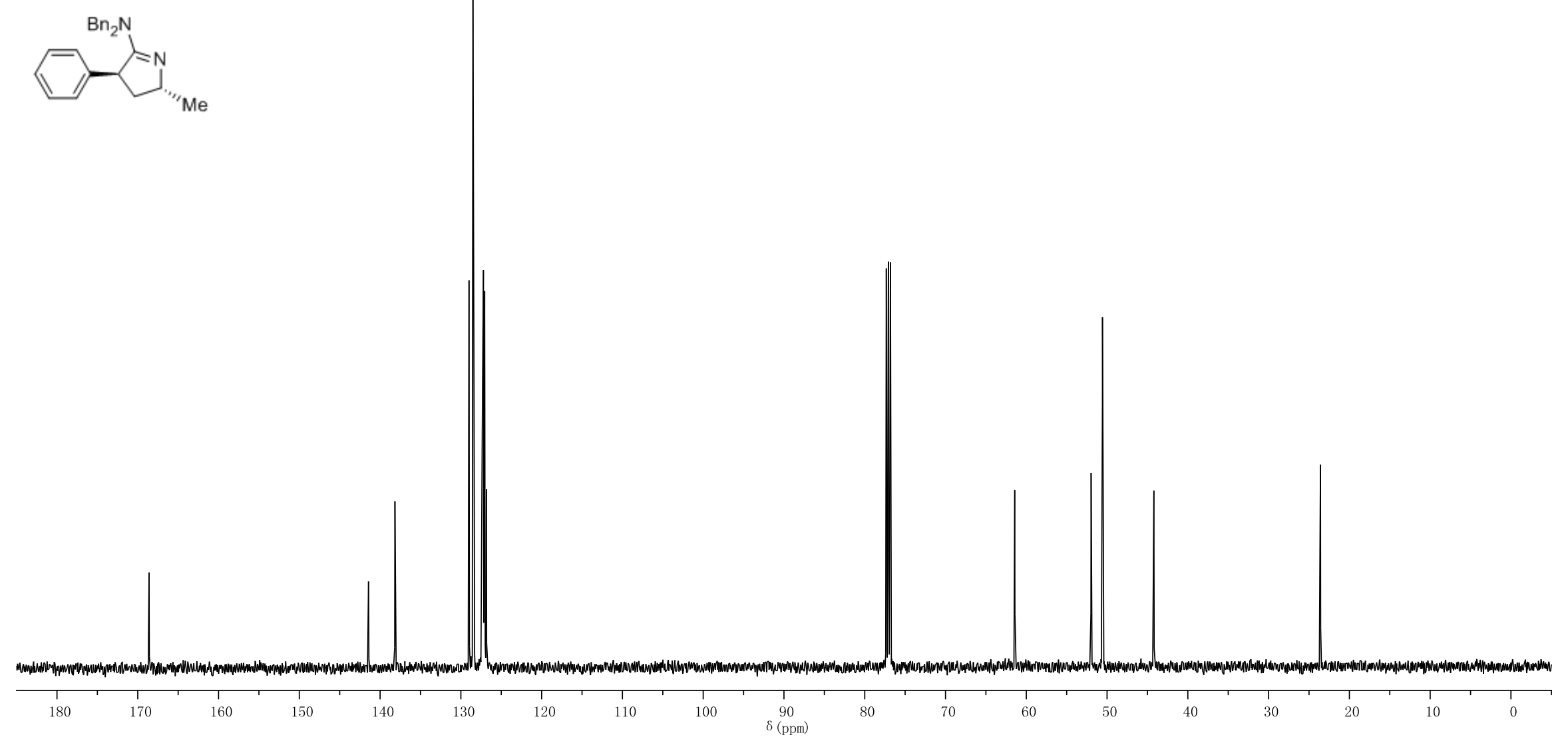


\section{Compound ( \pm )-3af}

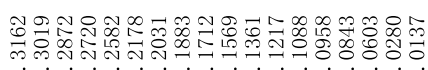

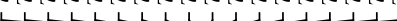

${ }^{1} \mathrm{H}$ NMR

$500 \mathrm{MHz}, \mathrm{CDCl}_{3}$
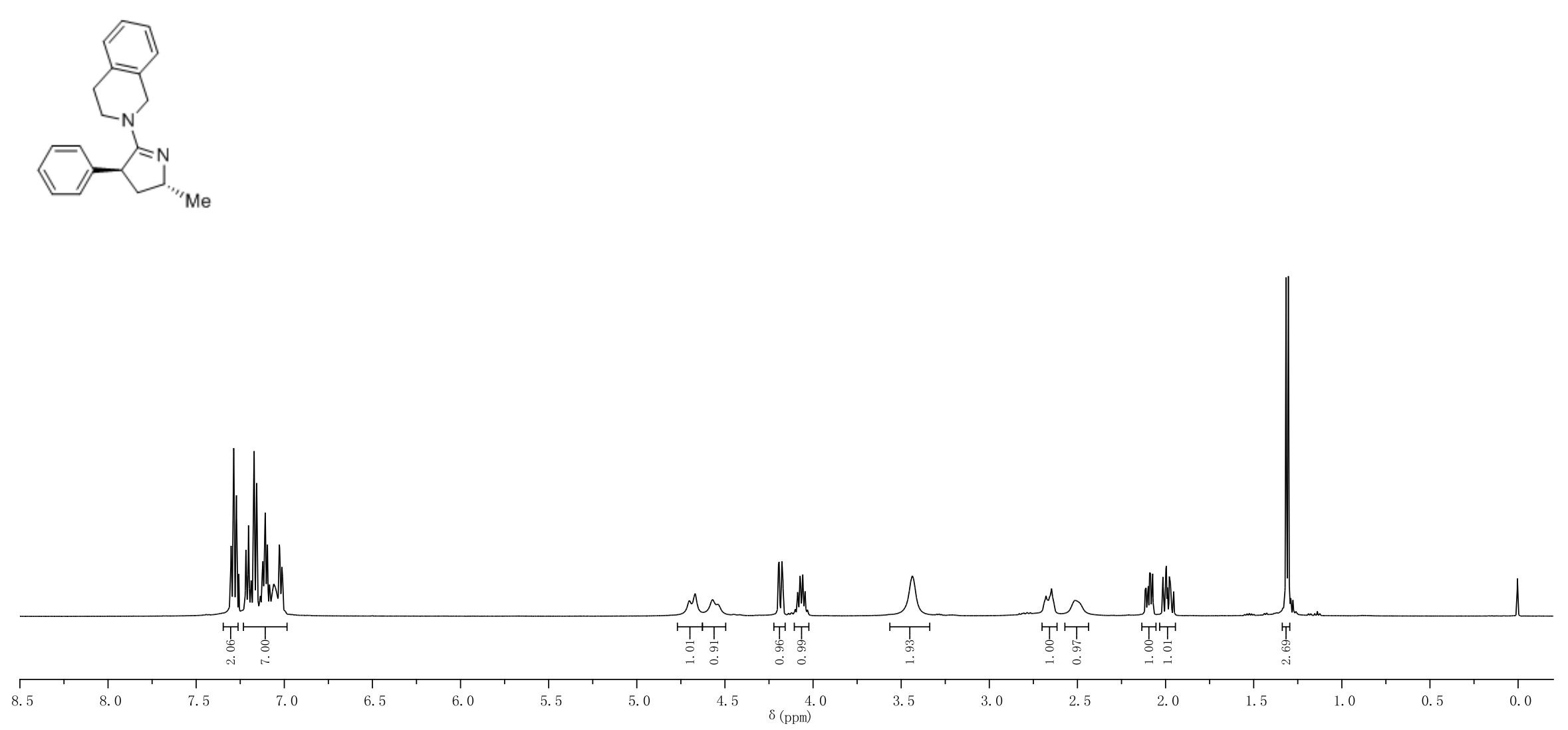
Compound ( \pm )-3af

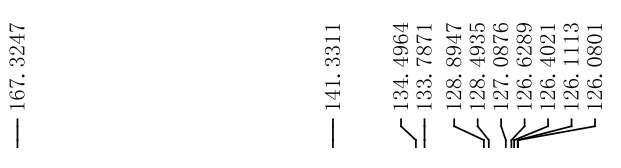

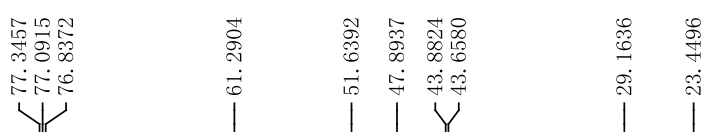

${ }^{13} \mathrm{C}\left\{{ }^{1} \mathrm{H}\right\}$ NMR

$125 \mathrm{MHz}, \mathrm{CDCl}_{3}$
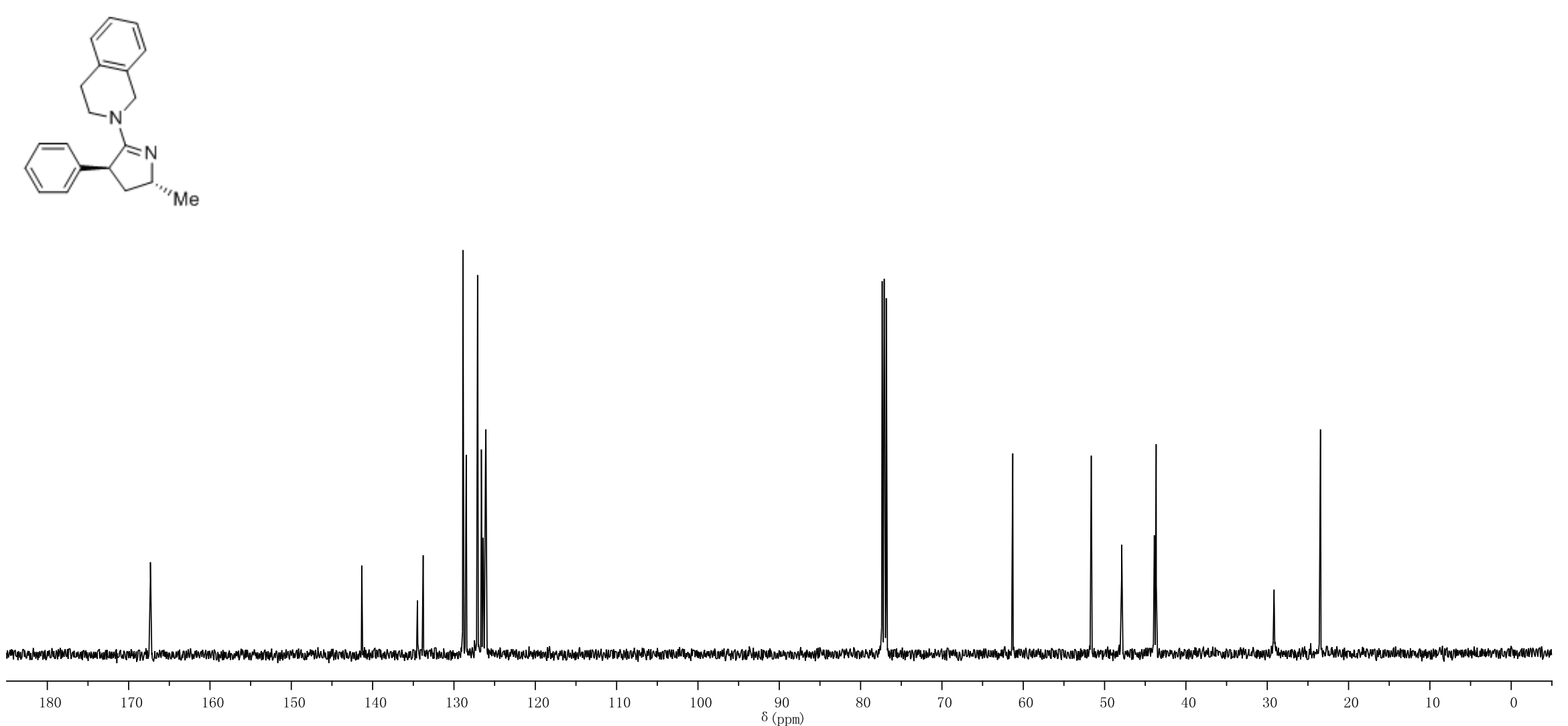


\section{Compound ( \pm )-3jg}

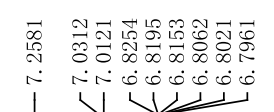

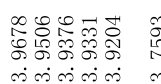

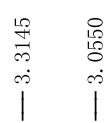

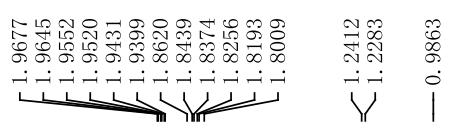

${ }^{1} \mathrm{H}$ NMR

$500 \mathrm{MHz}, \mathrm{CDCl}_{3}$
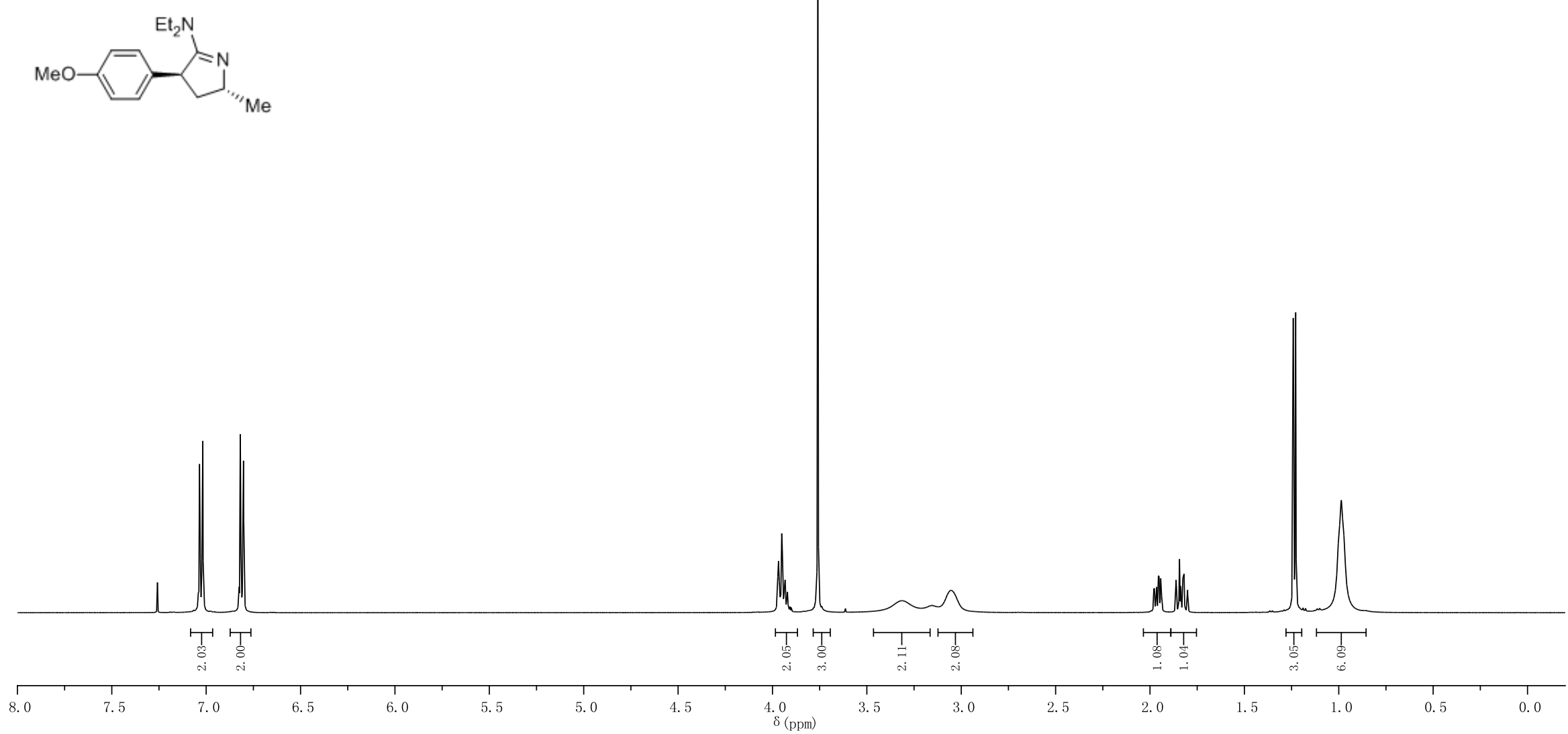


\section{Compound ( \pm -3jg}

|

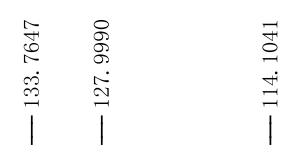

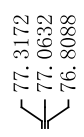

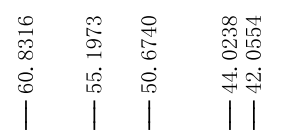

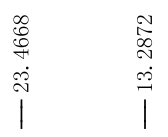

${ }^{13} \mathrm{C}\left\{{ }^{1} \mathrm{H}\right\}$ NMR

$125 \mathrm{MHz} \mathrm{CDCl}_{3}$
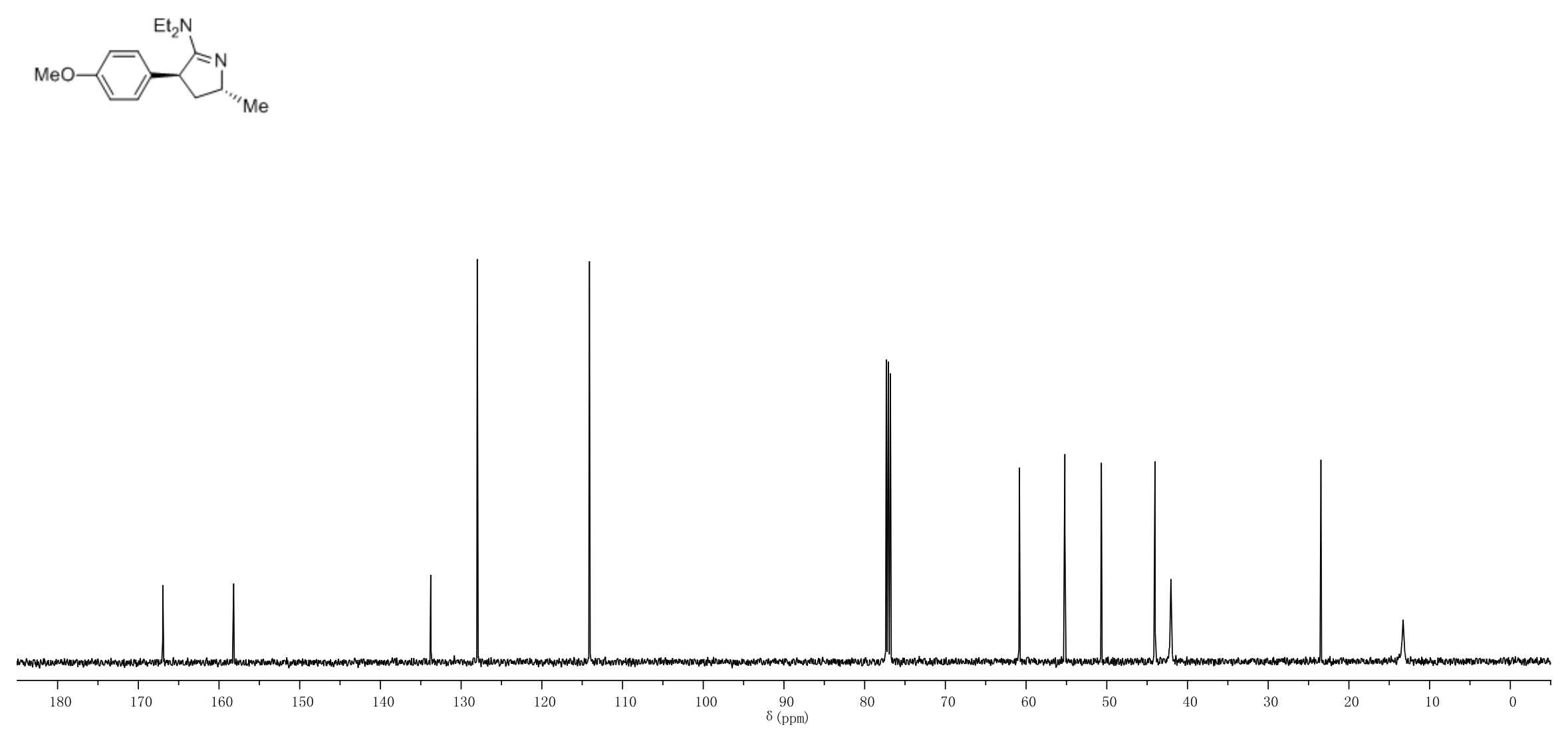


\section{Compound ( \pm )-3ah}

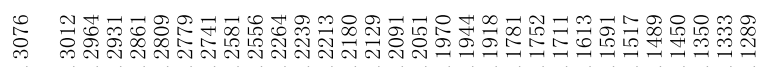

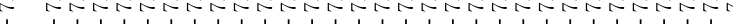

${ }^{1} \mathrm{H}$ NMR

$500 \mathrm{MHz}, \mathrm{CDCl}_{3}$
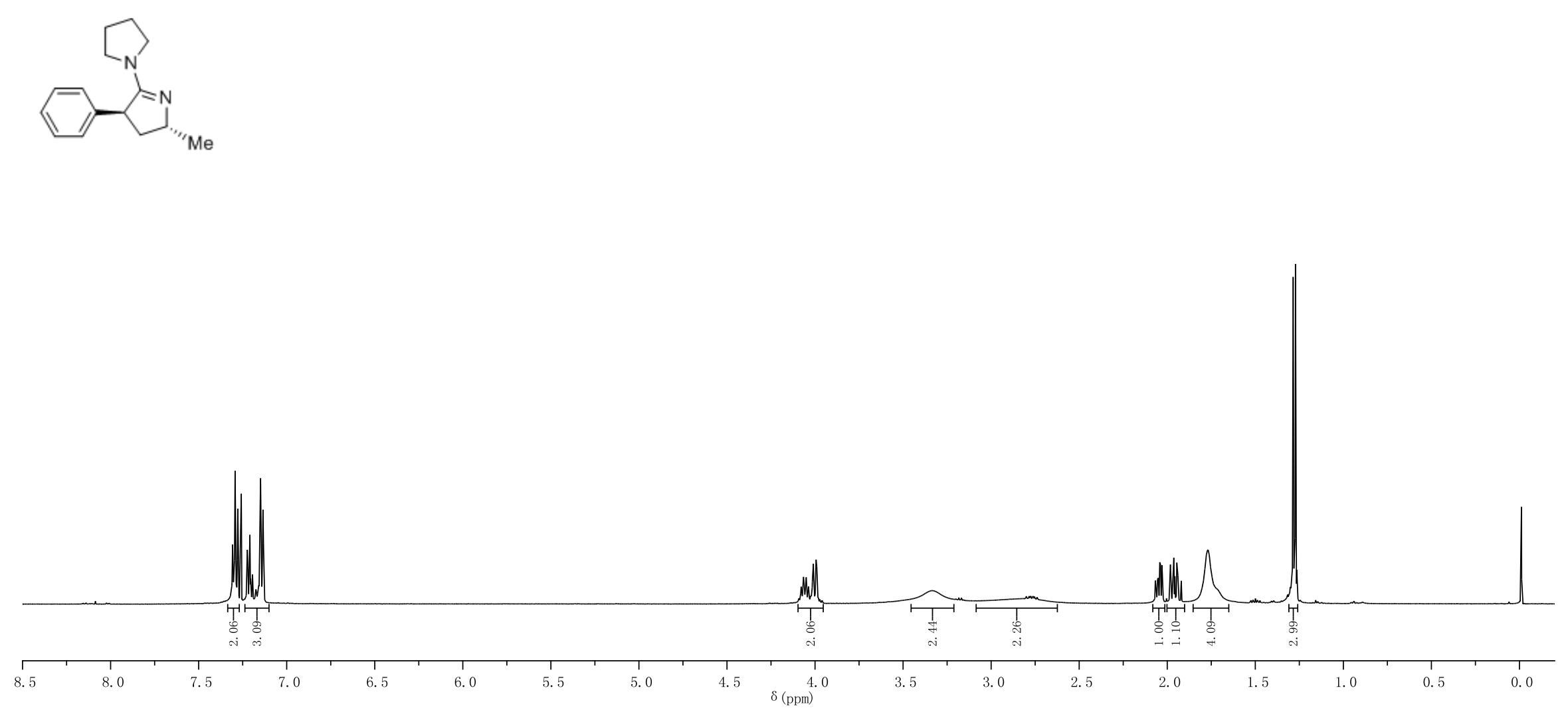

S169 
Compound ( \pm )-3ah

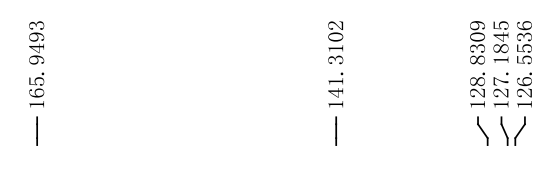

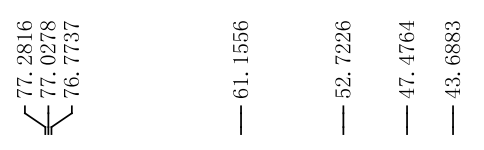

${ }^{13} \mathrm{C}\left\{{ }^{1} \mathrm{H}\right\}$ NMR

$125 \mathrm{MHz}, \mathrm{CDCl}_{3}$
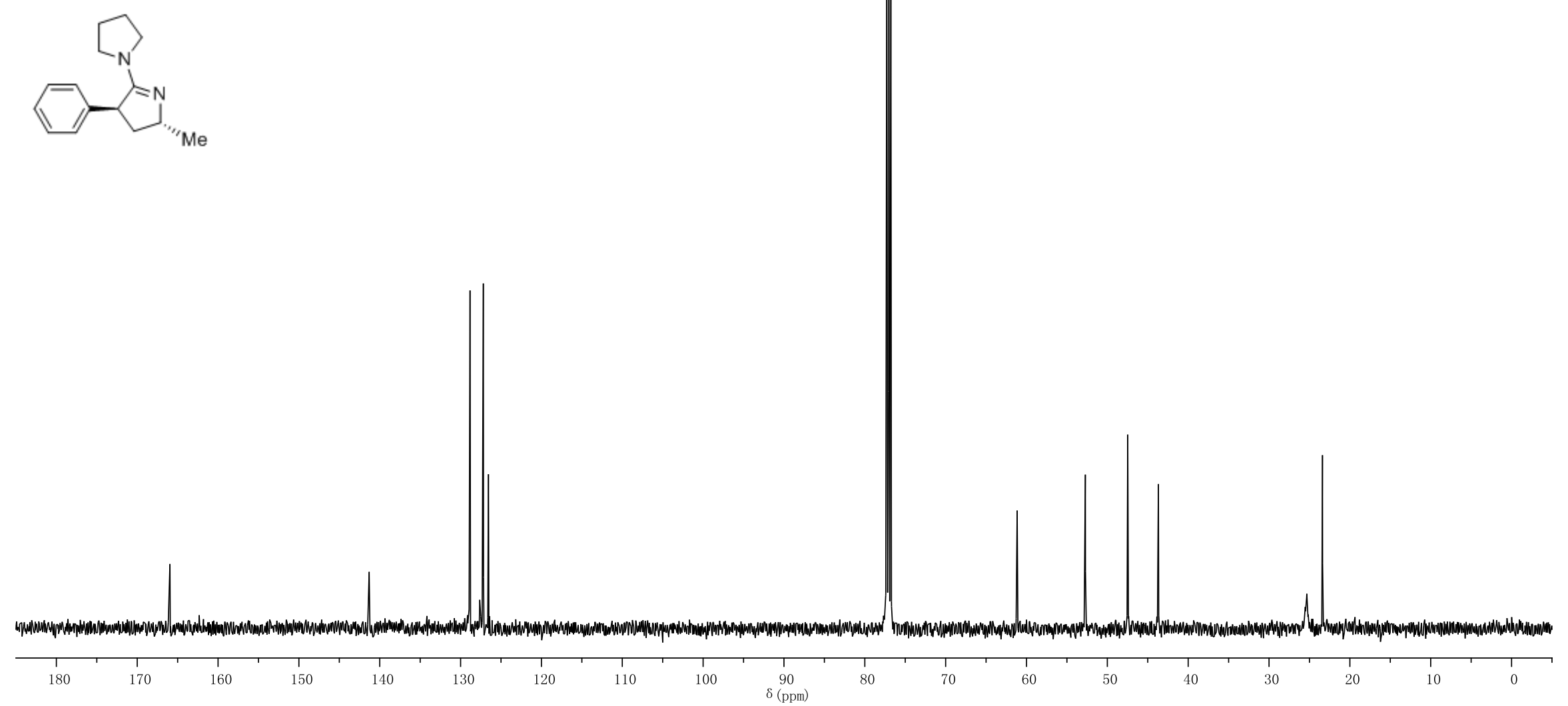


\section{Compound ( \pm )-3ai}

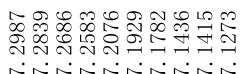

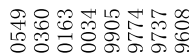

${ }^{1} \mathrm{H}$ NMR

$500 \mathrm{MHz}, \mathrm{CDCl}_{3}$
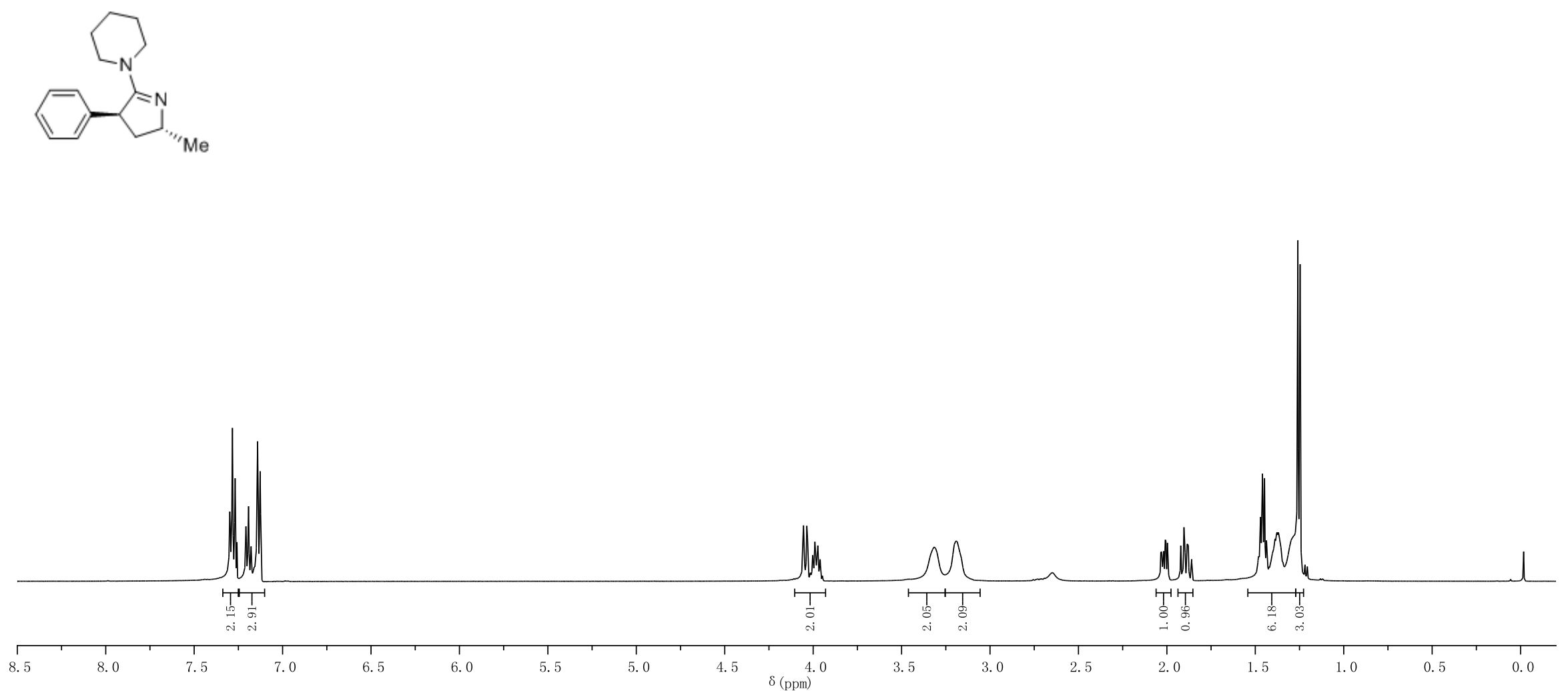
Compound ( \pm )-3ai

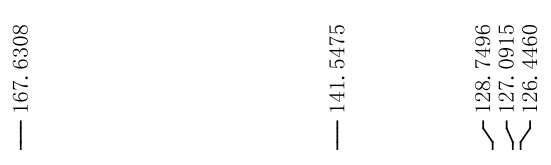

${ }^{13} \mathrm{C}\left\{{ }^{1} \mathrm{H}\right\}$ NMR

$125 \mathrm{MHz}, \mathrm{CDCl}_{3}$
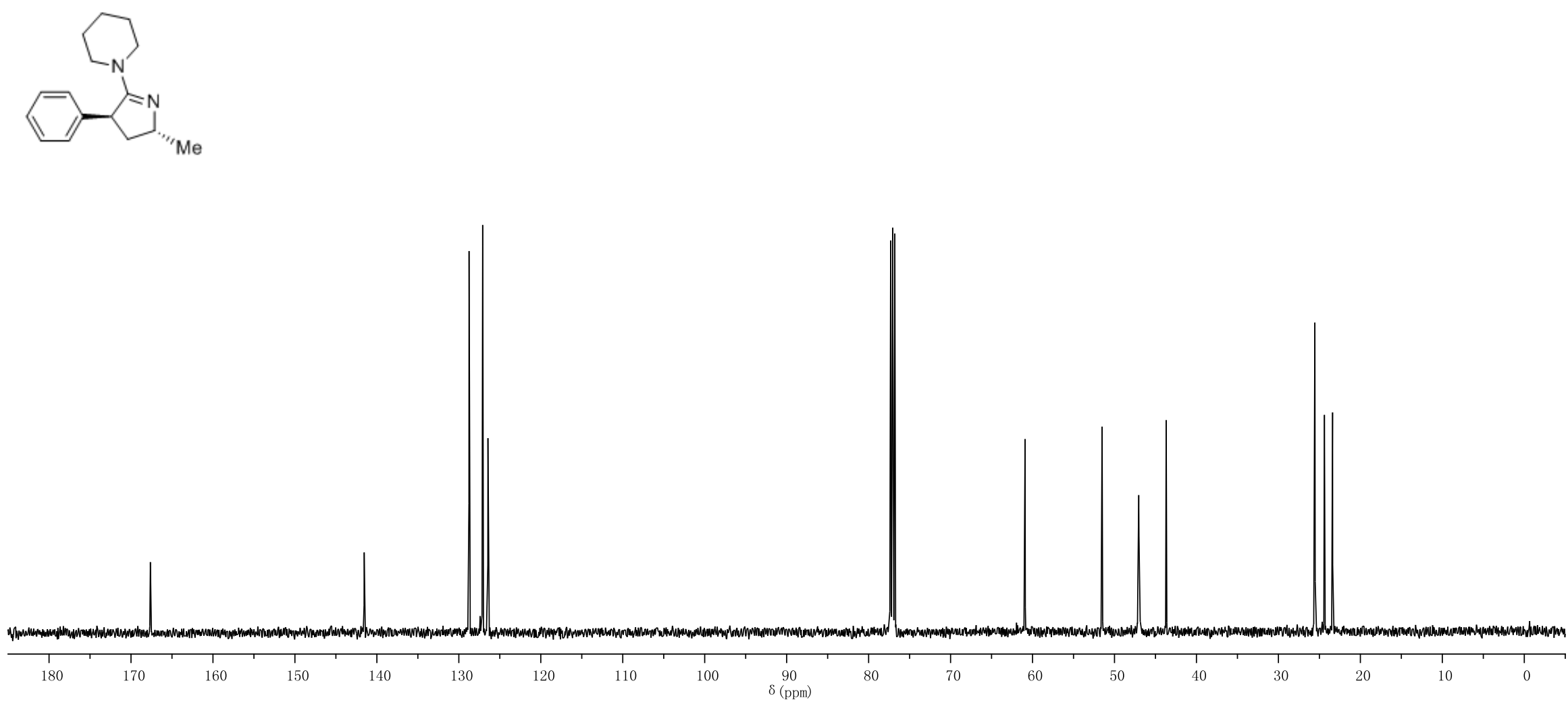


\section{Compound ( \pm$)-3 \mathbf{j j}$}

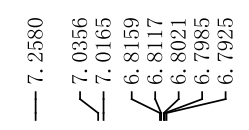

${ }^{1} \mathrm{H}$ NMR

$500 \mathrm{MHz}, \mathrm{CDCl}_{3}$
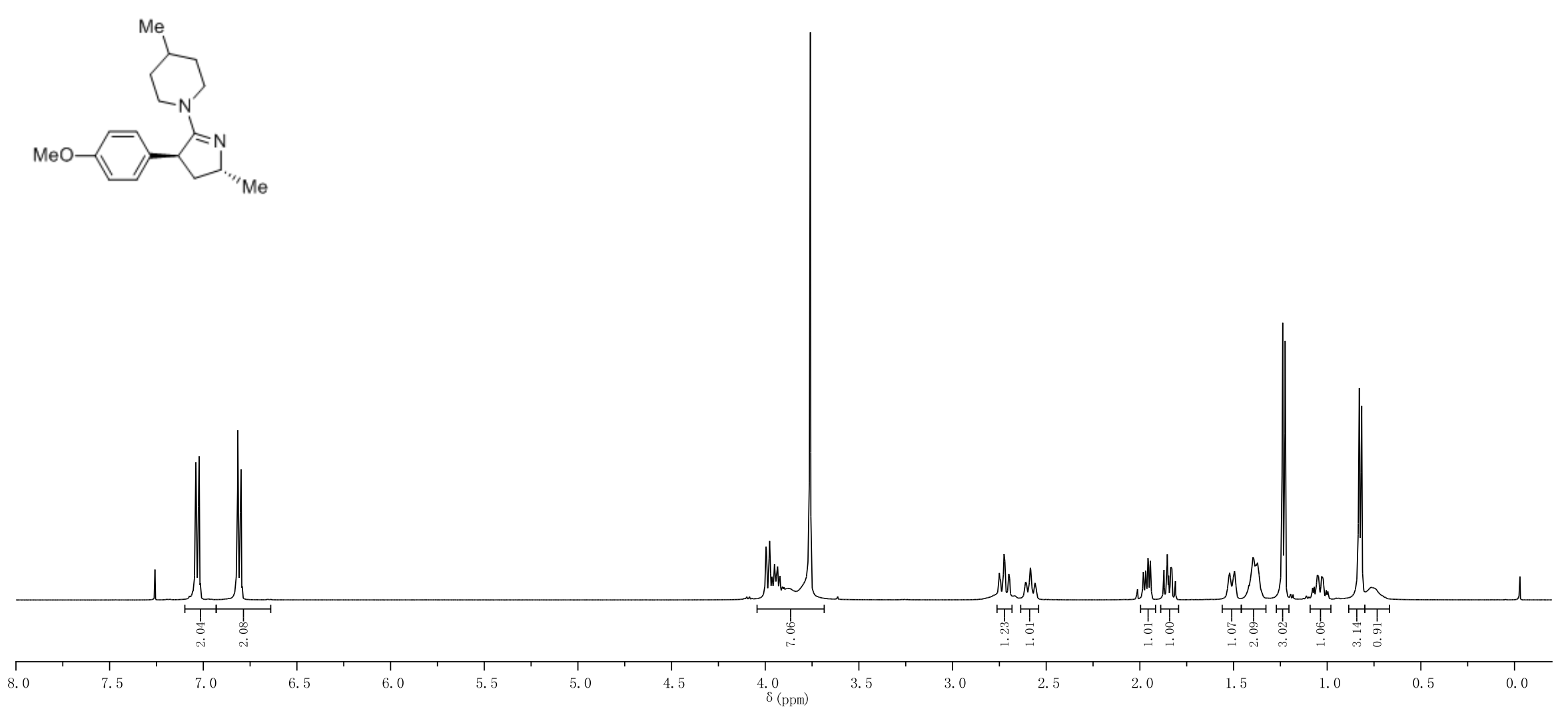


\section{Compound ( \pm )-3jj}

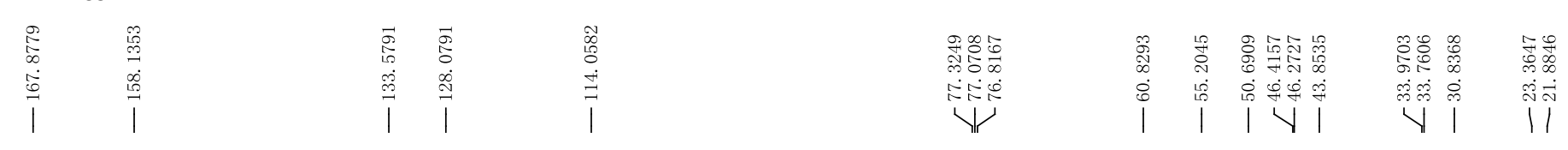

${ }^{13} \mathrm{C}\left\{{ }^{1} \mathrm{H}\right\} \mathrm{NMR}$

$125 \mathrm{MHz}, \mathrm{CDCl}_{3}$

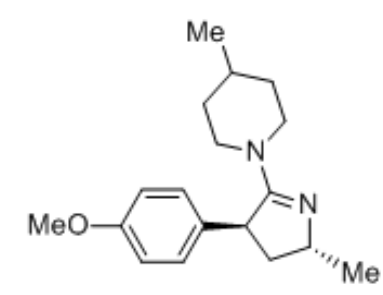

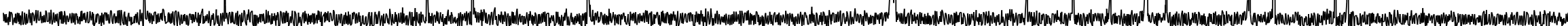

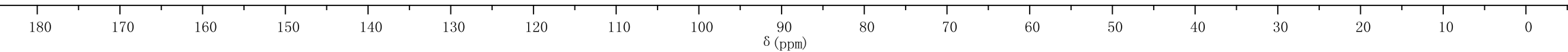




\section{Compound ( \pm )-3jk}

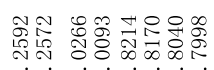

บับข้

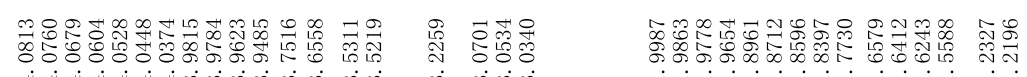

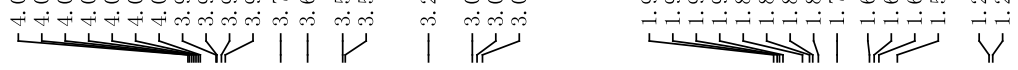

\section{${ }^{1} \mathrm{H}$ NMR}

$500 \mathrm{MHz}, \mathrm{CDCl}_{3}$
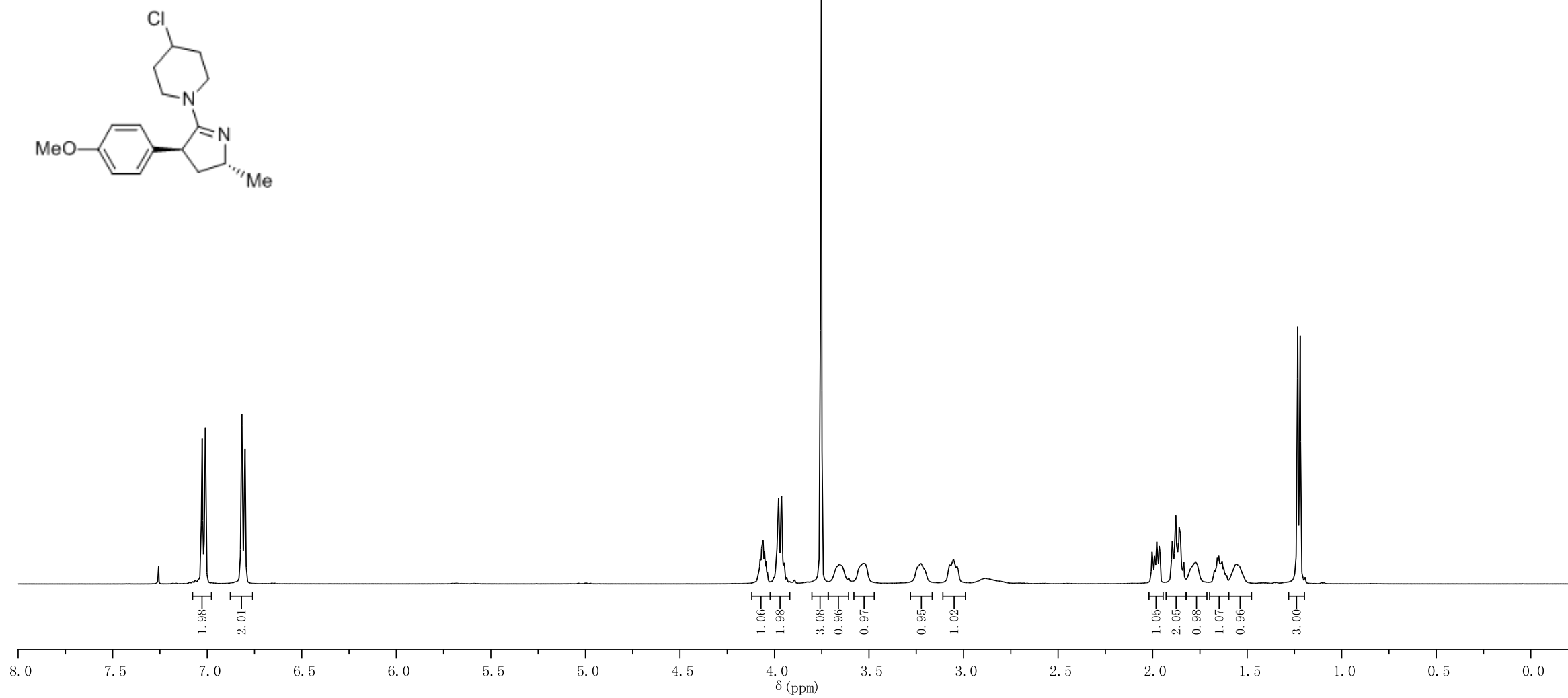


\section{Compound ( \pm )-3jk}

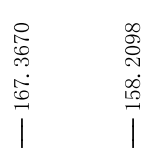

${ }^{13} \mathrm{C}\left\{{ }^{1} \mathrm{H}\right\} \mathrm{NMR}$

$125 \mathrm{MHz}, \mathrm{CDCl}_{3}$

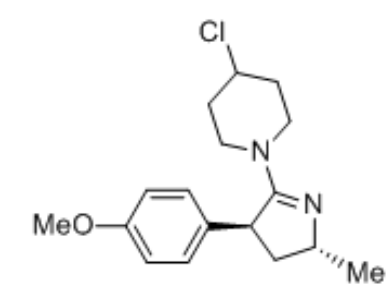

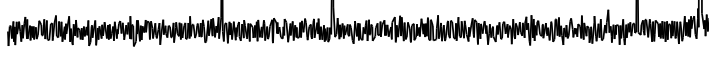

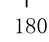

$170 \quad 160$

150

140

130

120

110

100 $\delta(\mathrm{ppm})$

80

70

40

30

20 


\section{Compound ( \pm$)-3 \mathbf{j l}$}
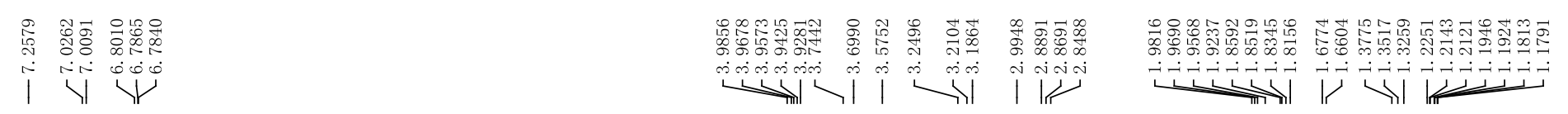

${ }^{1} \mathrm{H}$ NMR

$500 \mathrm{MHz}, \mathrm{CDCl}_{3}$
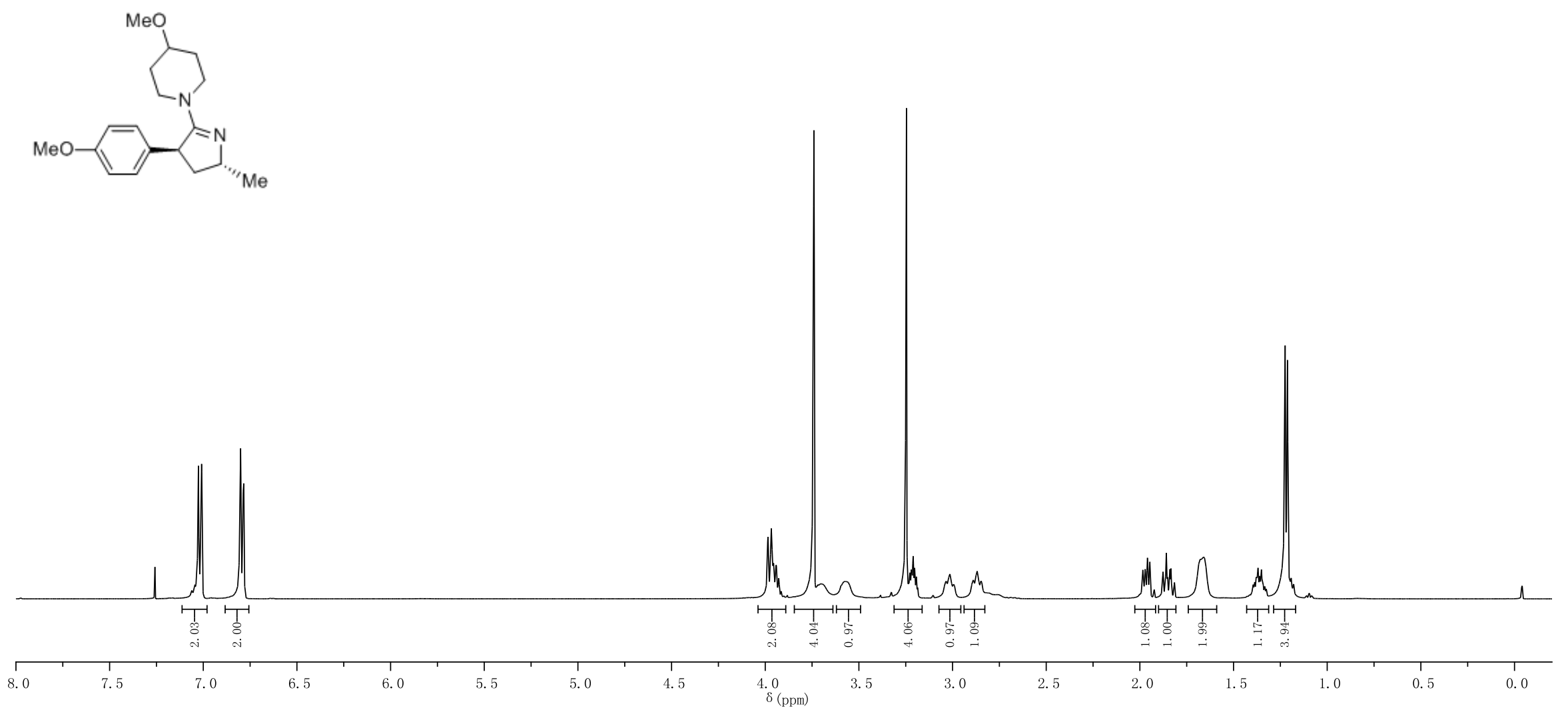


\section{Compound ( \pm )-3jl}

|

${ }^{13} \mathrm{C}\left\{{ }^{1} \mathrm{H}\right\}$ NMR

$125 \mathrm{MHz}, \mathrm{CDCl}_{3}$
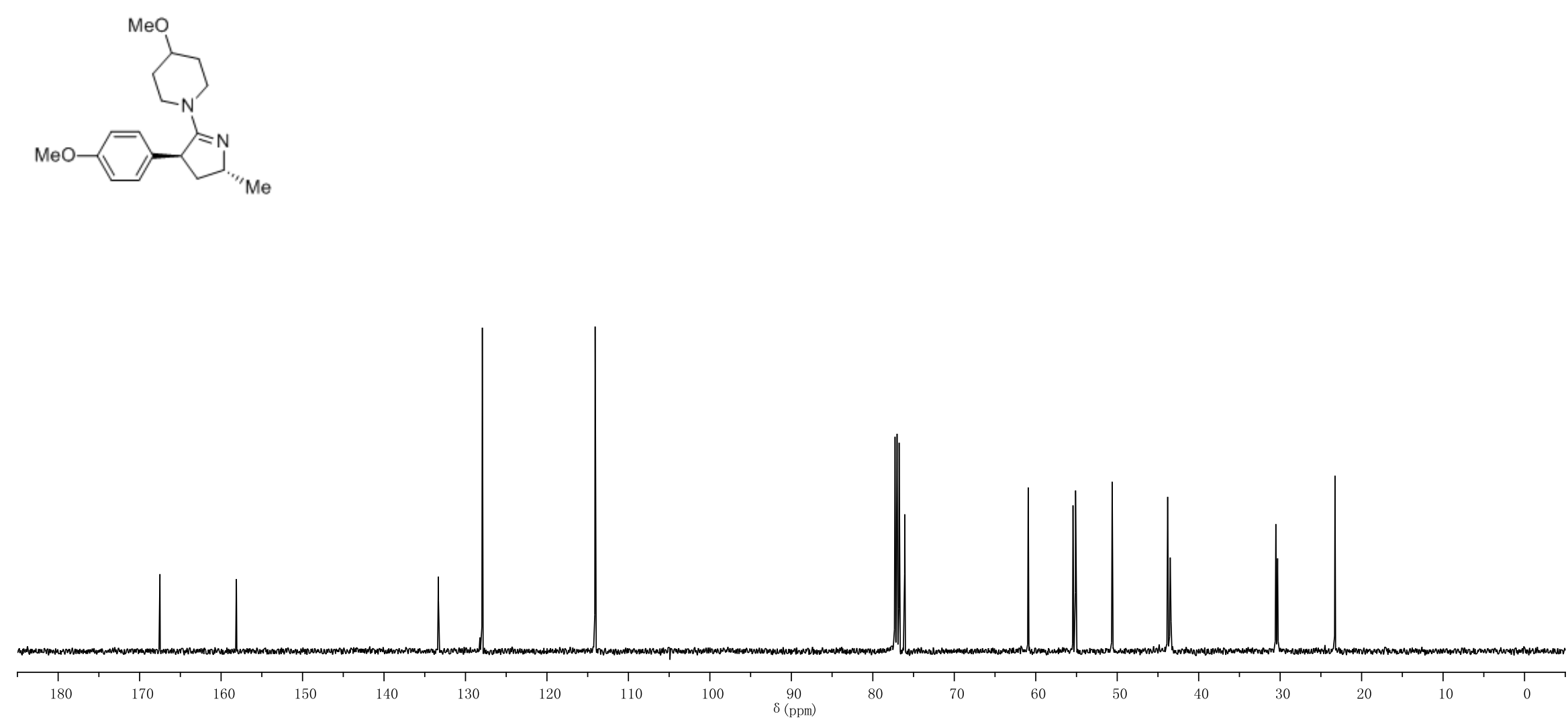


\section{Compound ( \pm )-3jm}

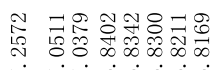

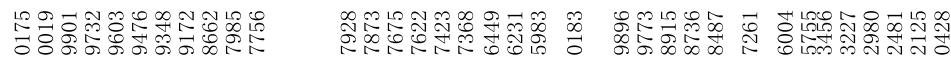

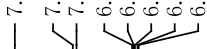

rimencment

${ }^{1} \mathrm{H}$ NMR

$500 \mathrm{MHz}, \mathrm{CDCl}_{3}$
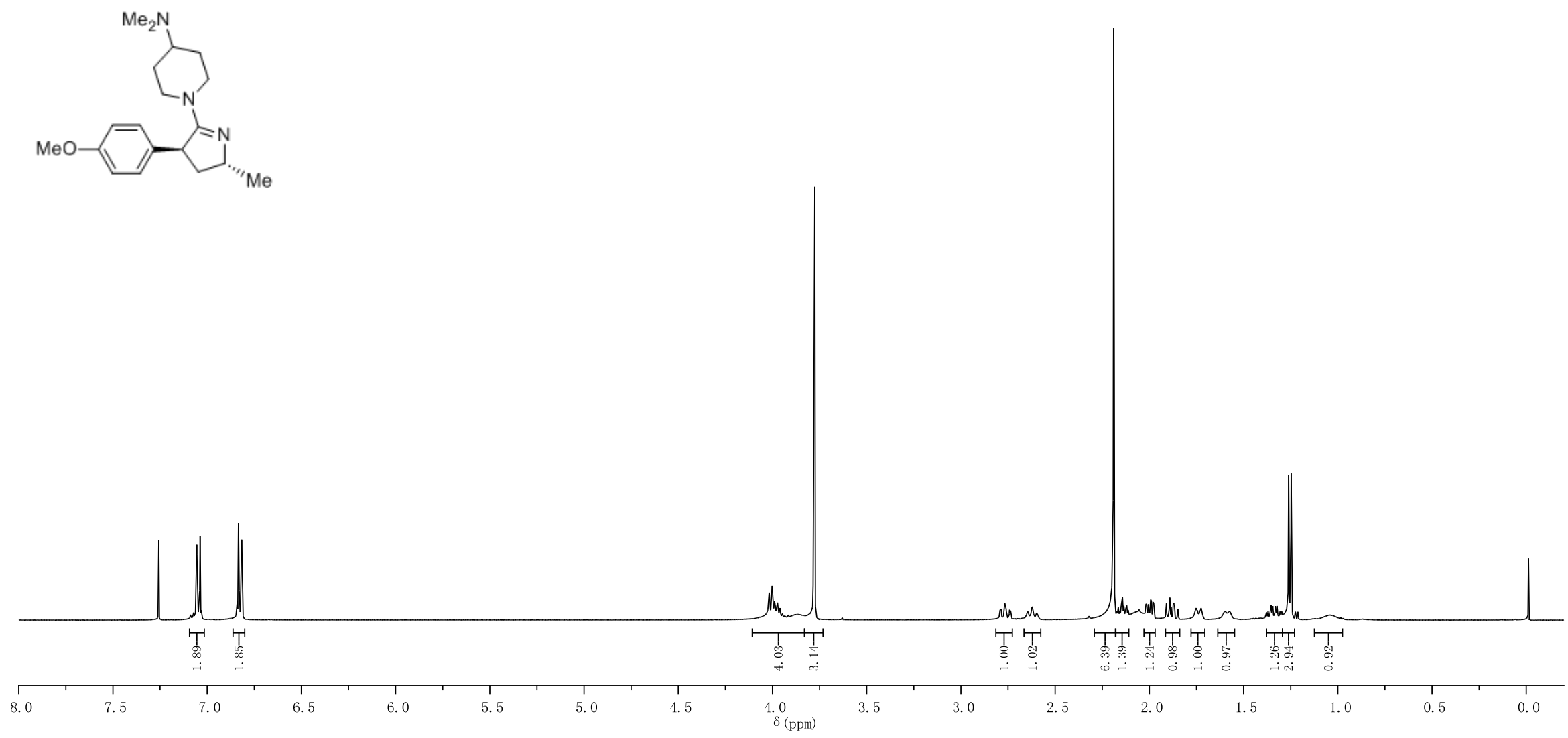


\section{Compound ( \pm )-3jm}

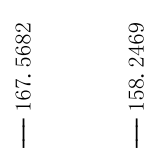

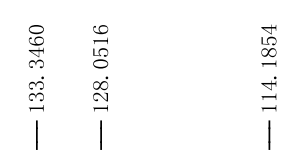

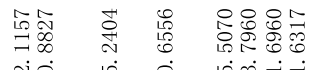

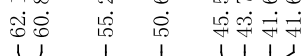

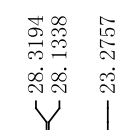

${ }^{13} \mathrm{C}\left\{{ }^{1} \mathrm{H}\right\}$ NMR

$125 \mathrm{MHz}, \mathrm{CDCl}_{3}$
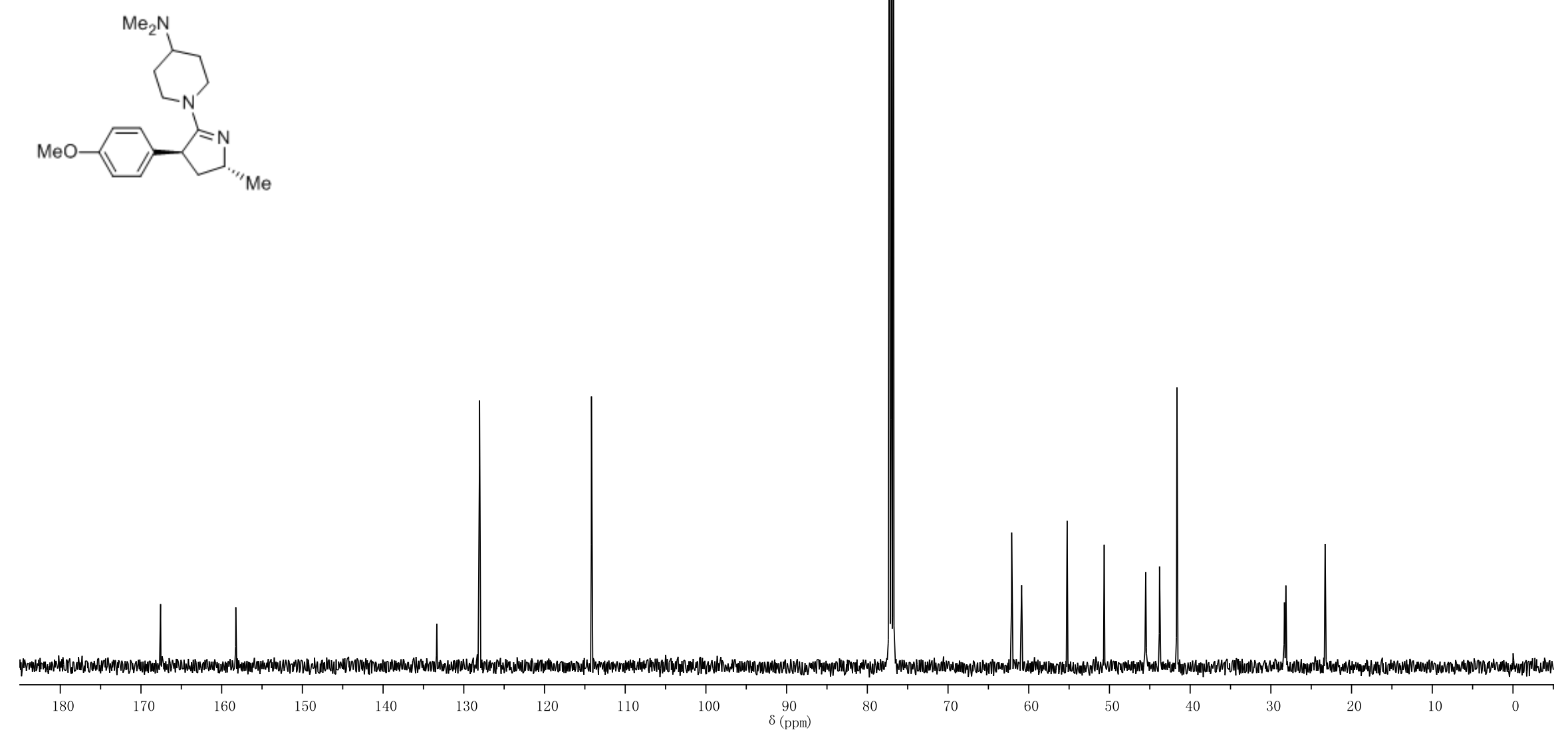


\section{Compound ( \pm )-3an}

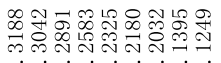

造证然

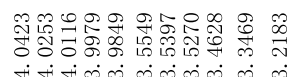

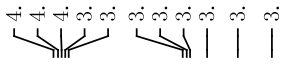

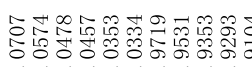

Vै

$500 \mathrm{MHz}, \mathrm{CDCl}_{3}$
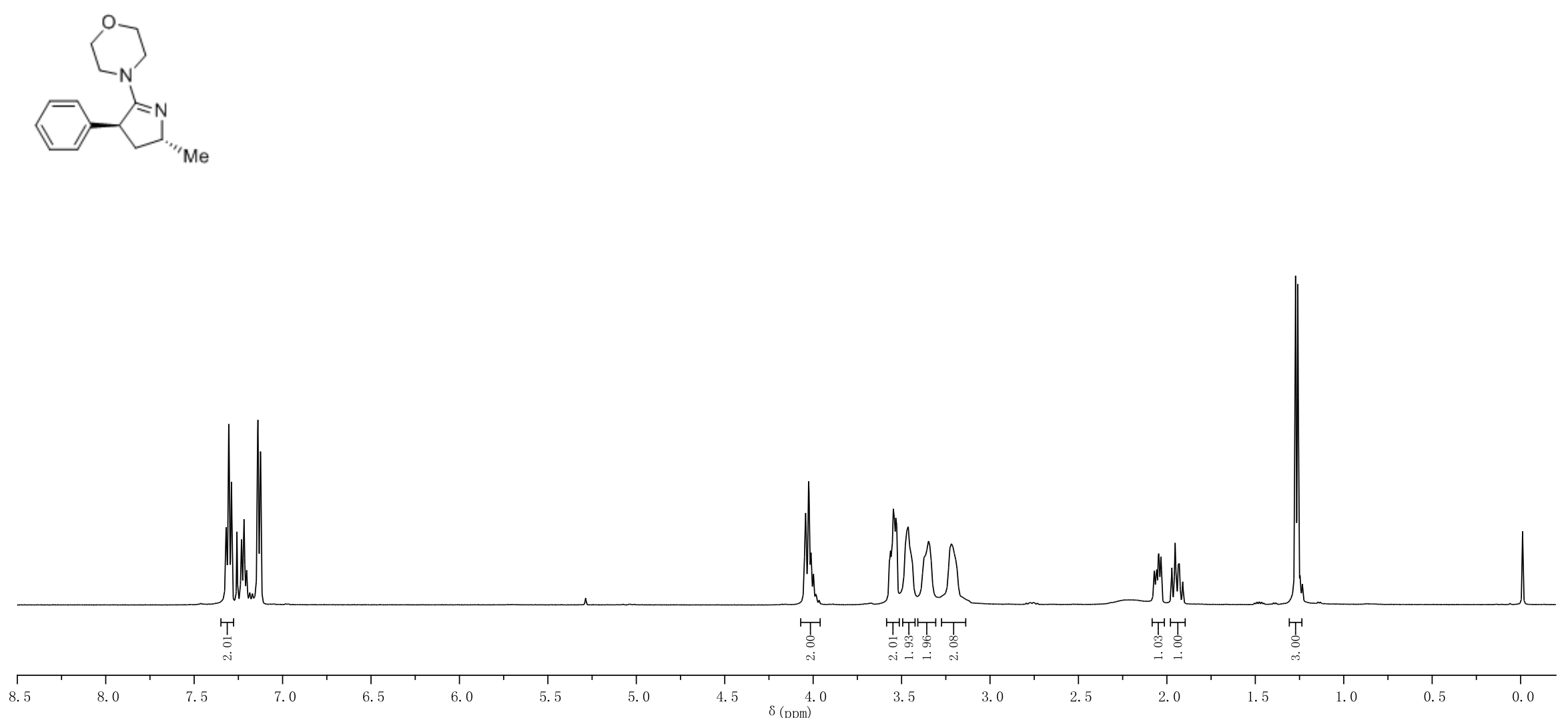
Compound ( \pm )-3an

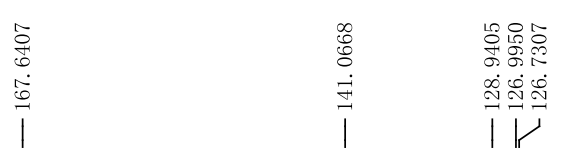

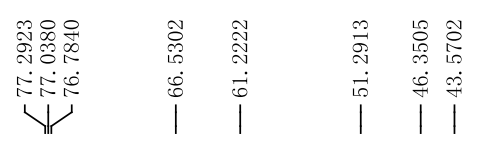

${ }^{13} \mathrm{C}\left\{{ }^{1} \mathrm{H}\right\}$ NMR

$125 \mathrm{MHz}, \mathrm{CDCl}_{3}$

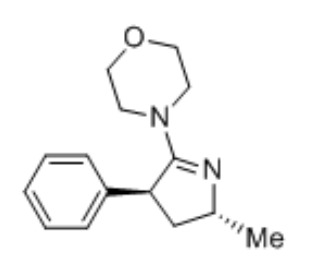

Me

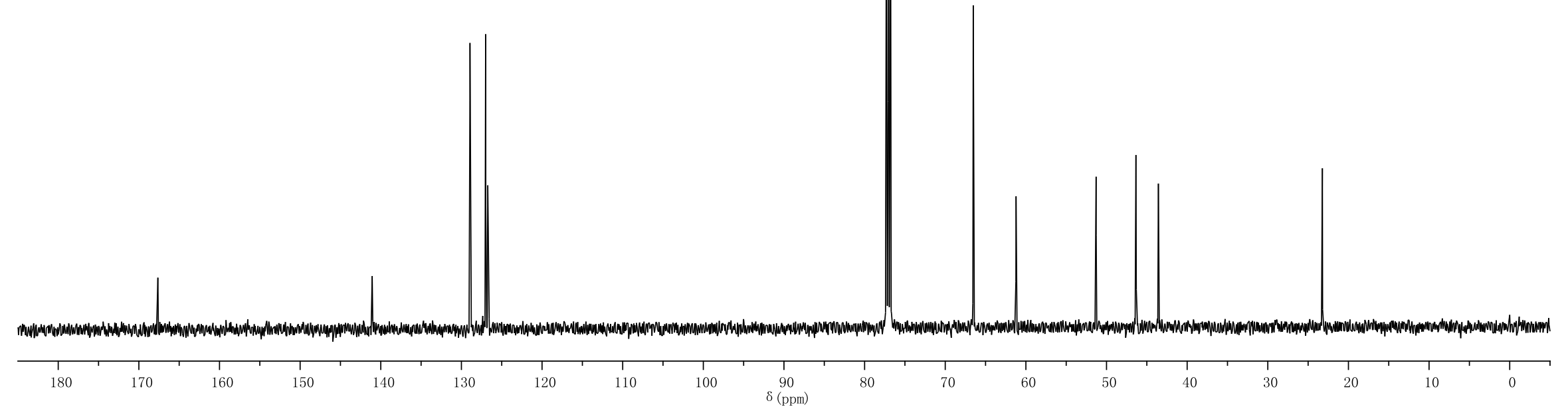



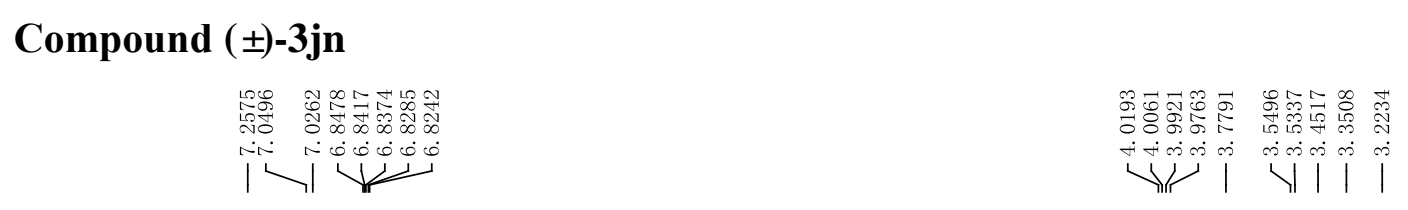

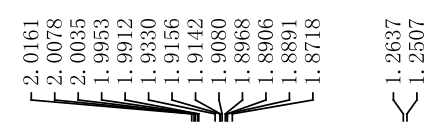

\section{${ }^{1} \mathrm{H}$ NMR}

$500 \mathrm{MHz}, \mathrm{CDCl}_{3}$
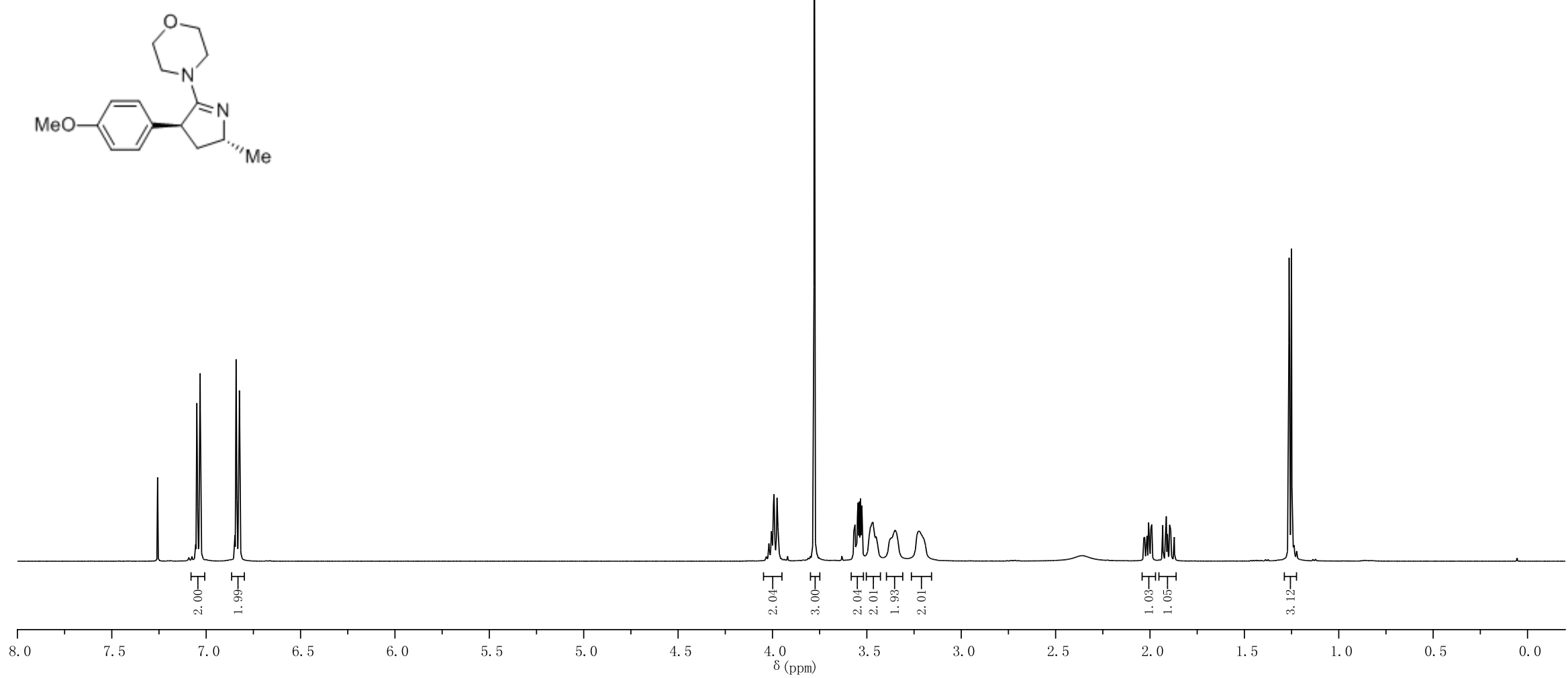


\section{Compound ( \pm )-3jn}

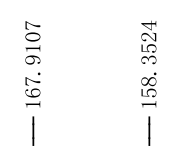

${ }^{13} \mathrm{C}\left\{{ }^{1} \mathrm{H}\right\}$ NMR

$125 \mathrm{MHz}, \mathrm{CDCl}_{3}$
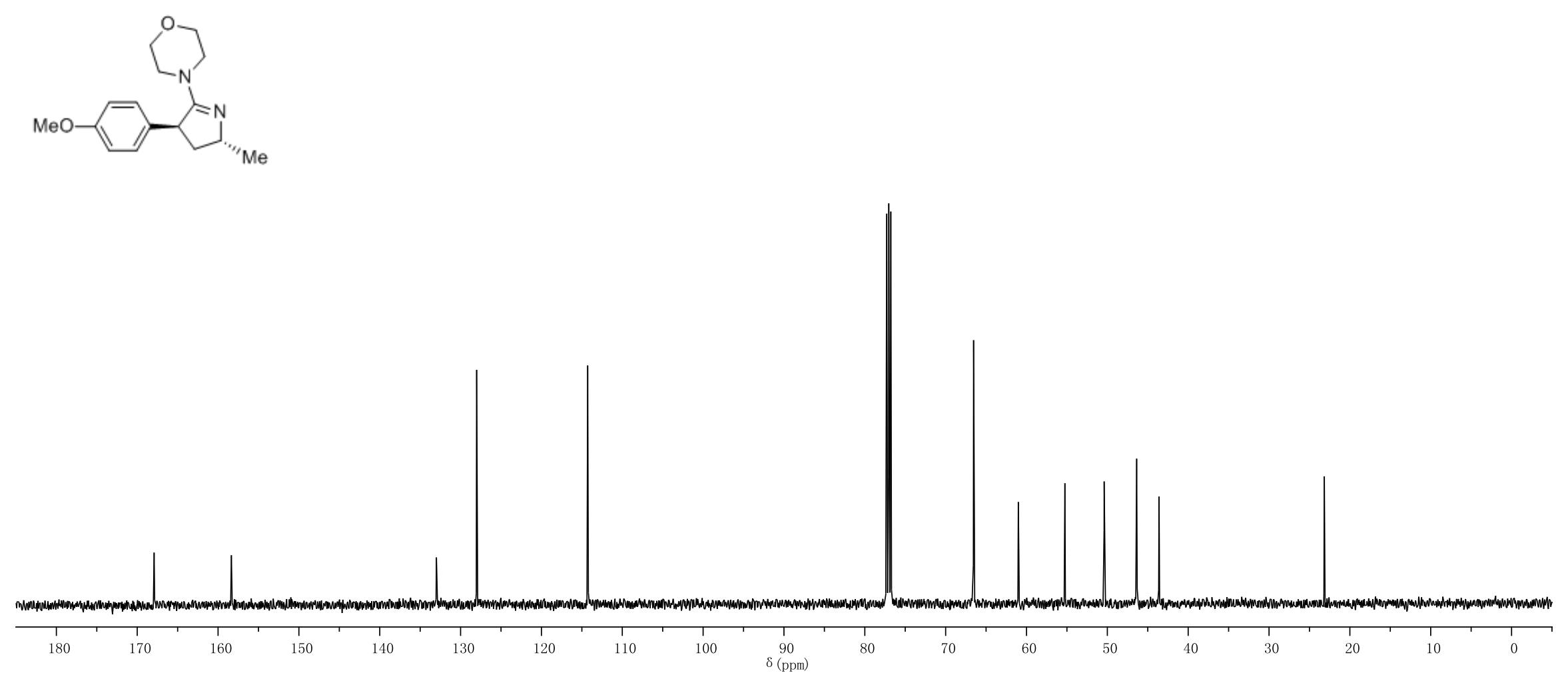


\section{Compound ( \pm )-3jo}

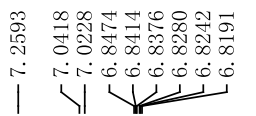

$500 \mathrm{MHz}, \mathrm{CDCl}_{3}$
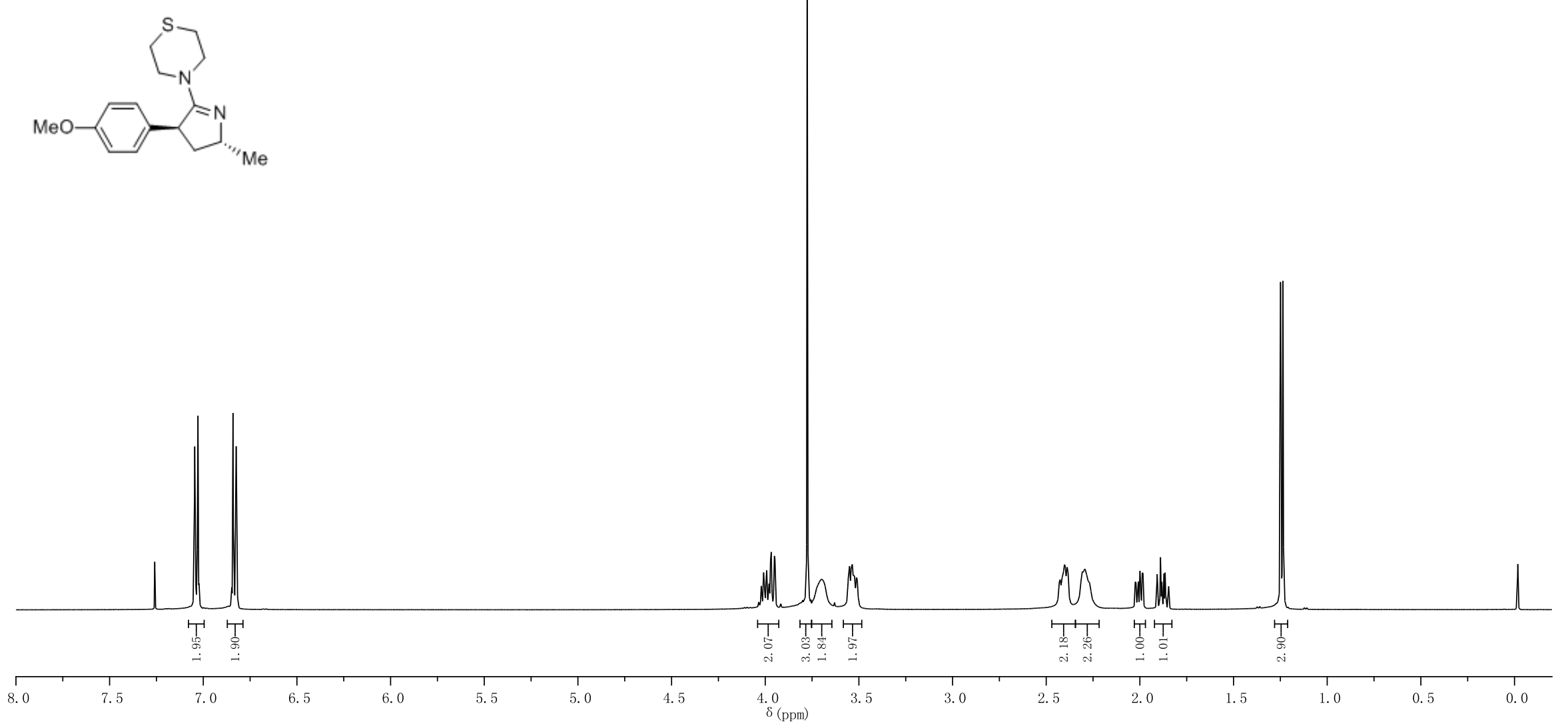


\section{Compound ( \pm$)-3$ jo}

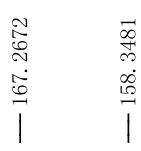

${ }^{13} \mathrm{C}\left\{{ }^{1} \mathrm{H}\right\}$ NMR

$125 \mathrm{MHz}, \mathrm{CDCl}_{3}$
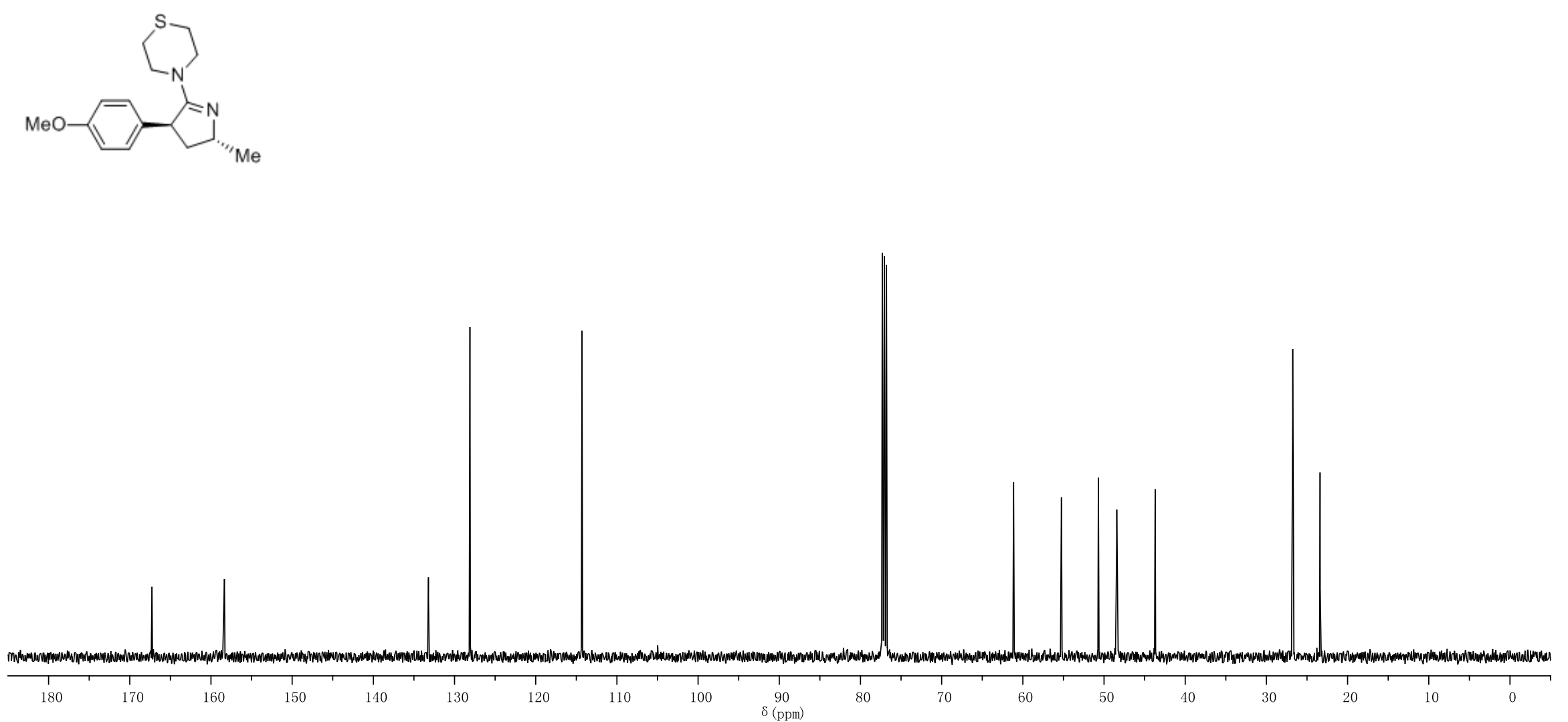


\section{Compound ( \pm )-3ap}

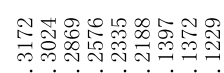

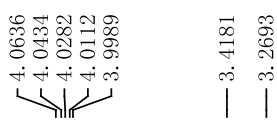

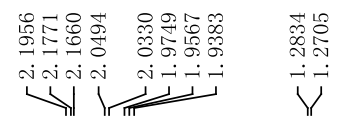

${ }^{1} \mathrm{H}$ NMR

$500 \mathrm{MHz}, \mathrm{CDCl}_{3}$
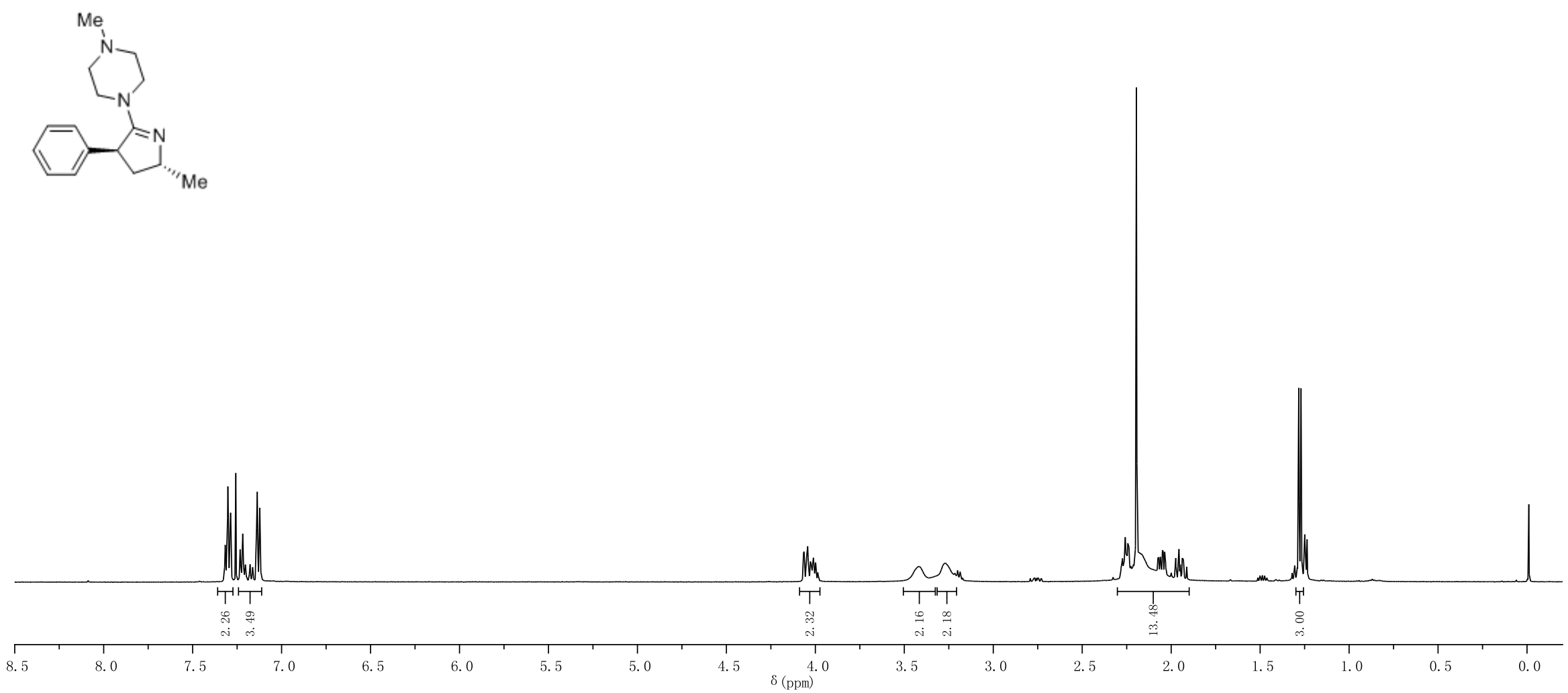
Compound ( \pm )-3ap

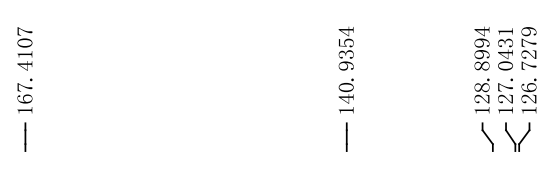

${ }^{13} \mathrm{C}\left\{{ }^{1} \mathrm{H}\right\}$ NMR

$125 \mathrm{MHz}, \mathrm{CDCl}_{3}$
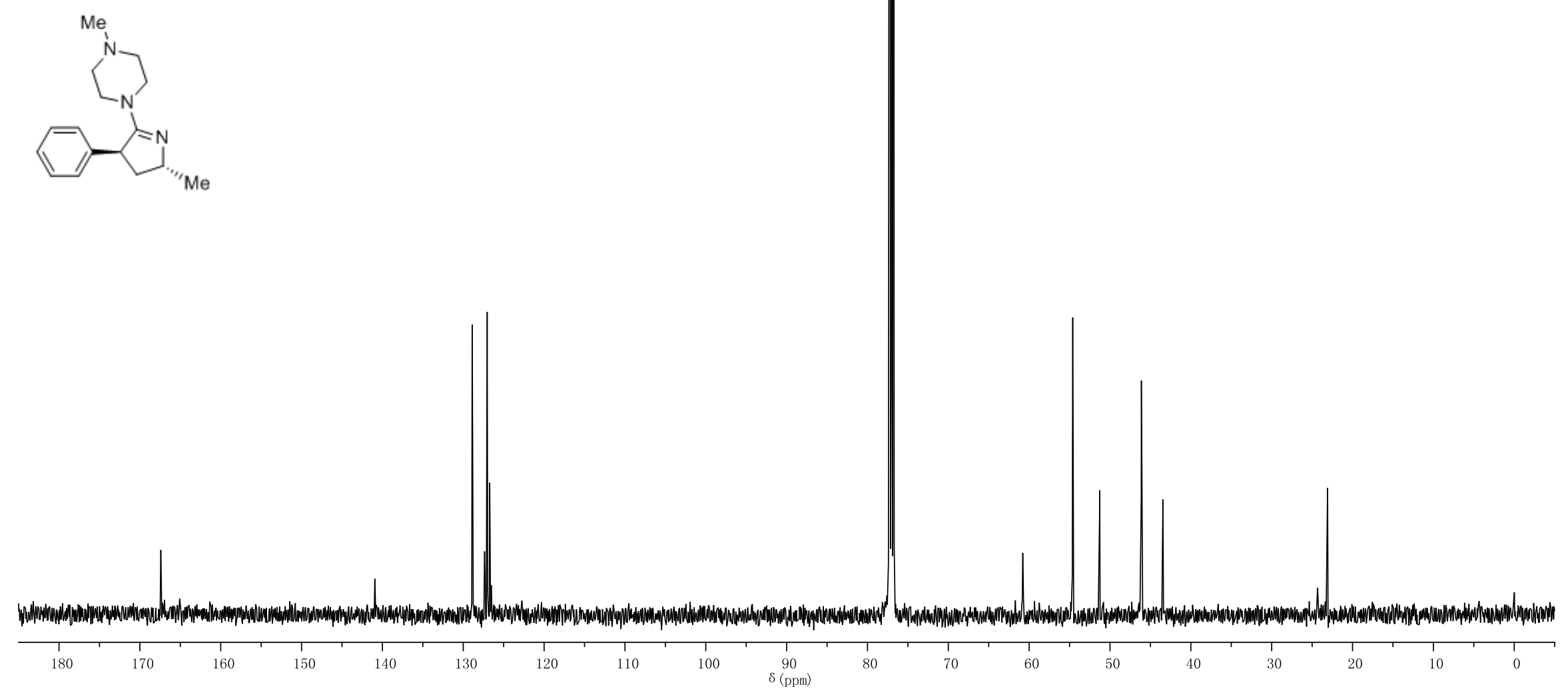


\section{Compound ( \pm )-3au}

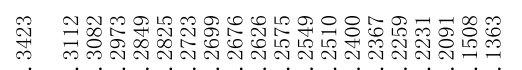

${ }^{1} \mathrm{H}$ NMR

$500 \mathrm{MHz}, \mathrm{CDCl}_{3}$

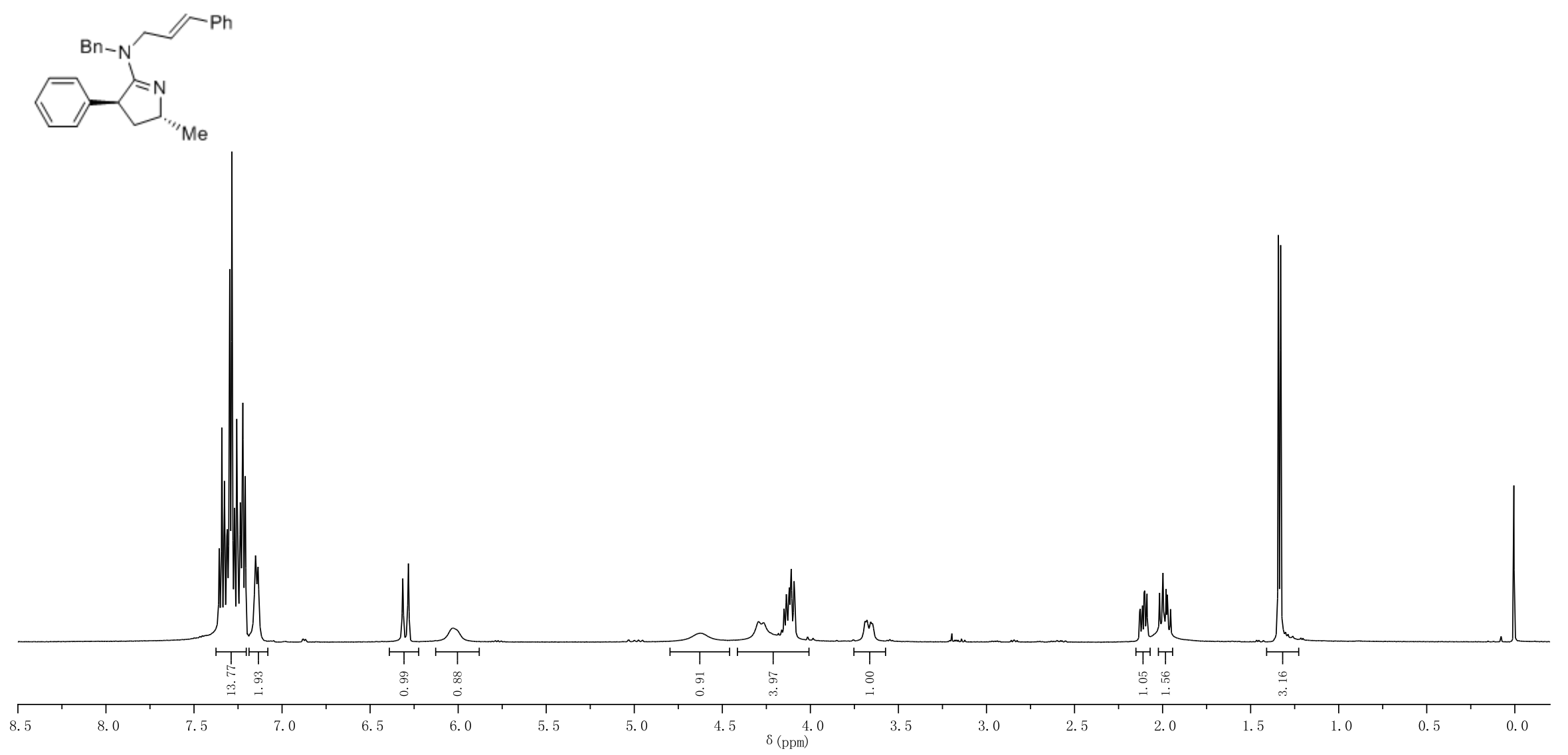


Compound ( \pm )-3au
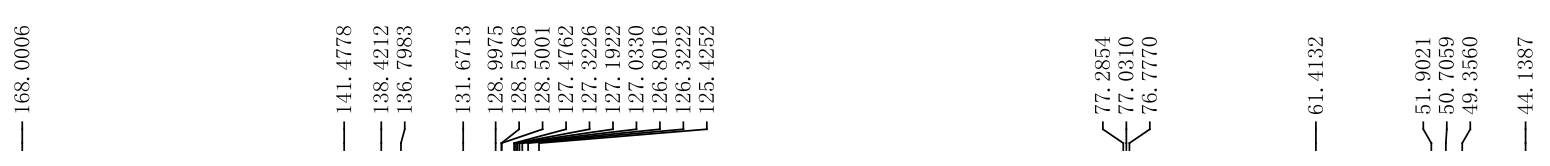

${ }^{13} \mathrm{C}\left\{{ }^{1} \mathrm{H}\right\}$ NMR

$125 \mathrm{MHz}, \mathrm{CDCl}_{3}$
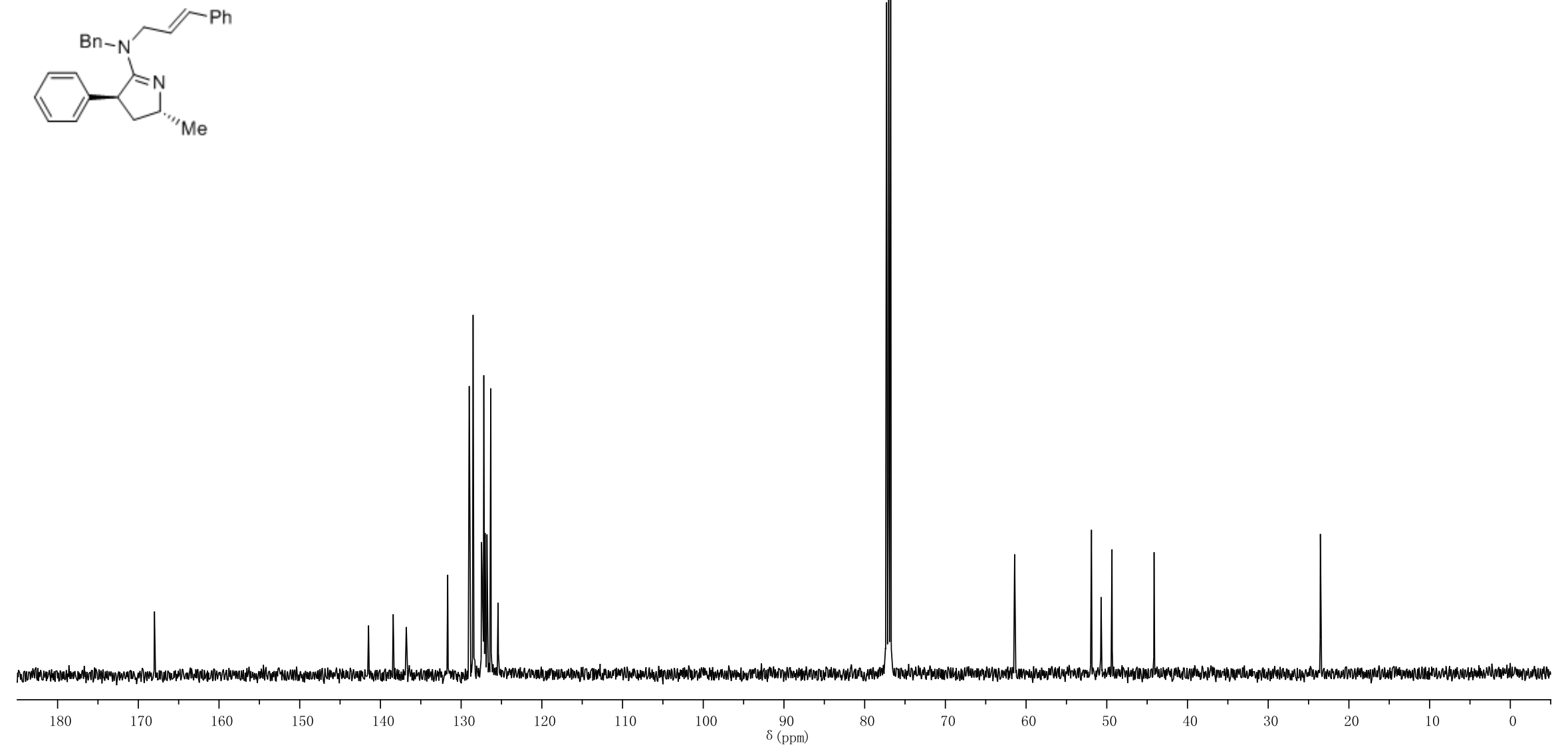


\section{Copies of NMR spectra for 4}

\section{Compound ( \pm )-4}

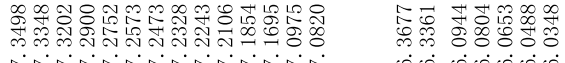

i.

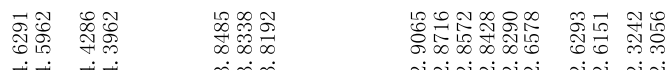

نं।

${ }^{1} \mathrm{H}$ NMR

$500 \mathrm{MHz}, \mathrm{CDCl}_{3}$
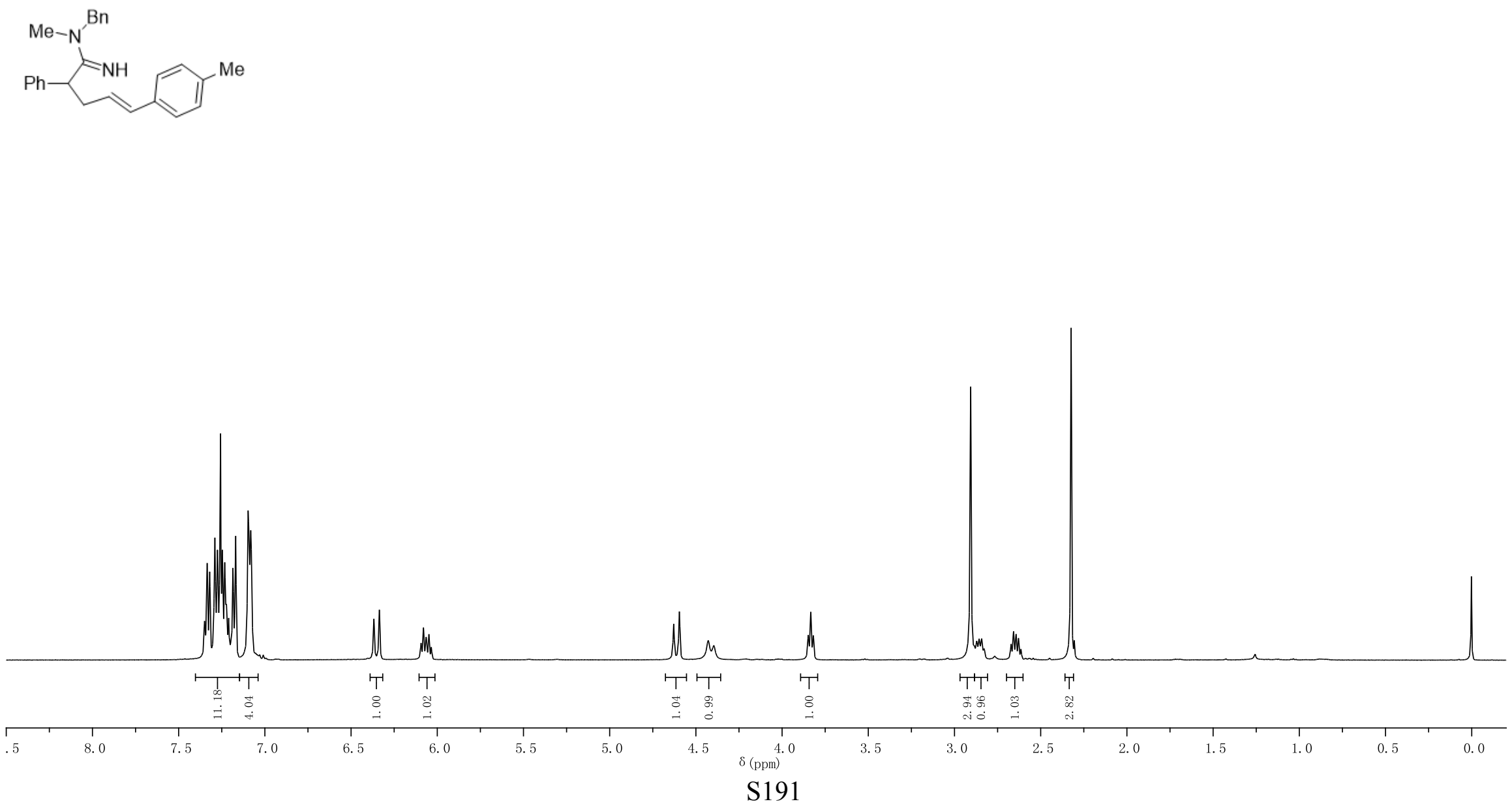


\section{Compound ( \pm )-4}

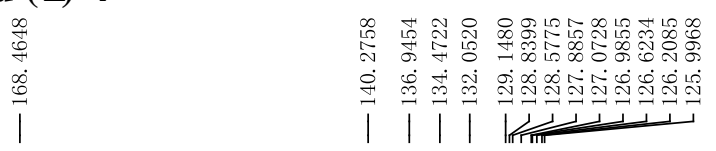

${ }^{13} \mathrm{C}\left\{{ }^{1} \mathrm{H}\right\}$ NMR

$125 \mathrm{MHz}, \mathrm{CDCl}_{3}$

$\mathrm{Me}-\mathrm{N}^{\mathrm{Bn}}$

Me

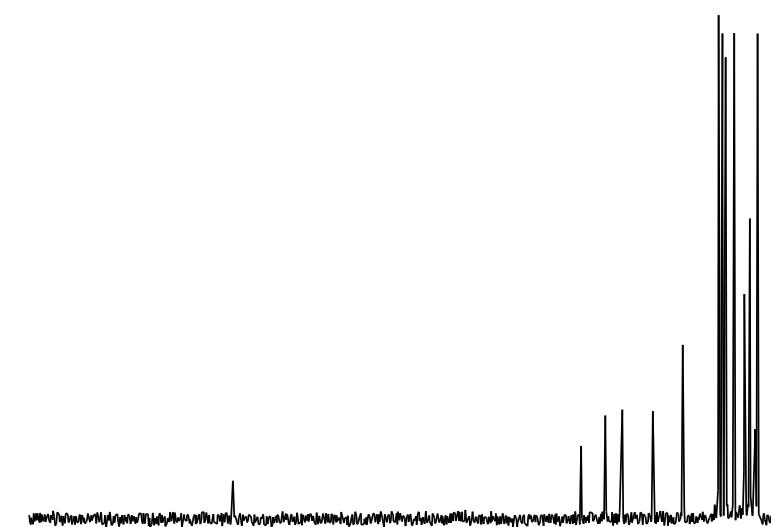

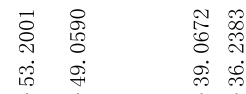

| 


\section{2. ${ }^{1} \mathrm{H}$ NMR Studies on the Mixing Order of the Catalyst and Reactants}

\section{Procedure A}

$\mathrm{Lu}\left(\mathrm{CH}_{2} \mathrm{C}_{6} \mathrm{H}_{4} \mathrm{NME}_{2}-\mathrm{O}\right)_{3}$ in $\mathrm{C}_{6} \mathrm{D}_{6}$ $\ln m$. m

MeNHBn in $\mathrm{C}_{6} \mathrm{D}_{6}$

2a

Mhum
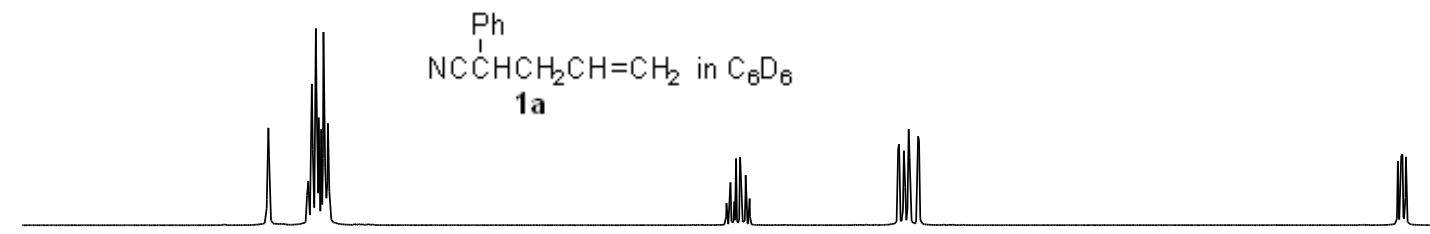

INA

$\mathrm{Ph}$

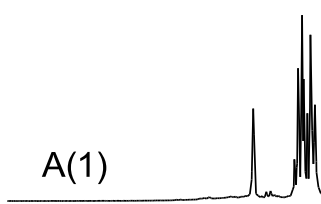

$\mathrm{NCC} \mathrm{CHCH}_{2} \mathrm{CH}=\mathrm{CH}_{2}+\mathrm{Lu}_{(}\left(\mathrm{CH}_{2} \mathrm{C}_{6} \mathrm{H}_{4} \mathrm{NMe}_{2}-\mathrm{O}\right)_{3}$ in $\mathrm{C}_{6} \mathrm{D}_{6}$, for 20 min at $25^{\circ} \mathrm{C}$ $0.1 \mathrm{mmol}$ $0.005 \mathrm{mmol}$ IN
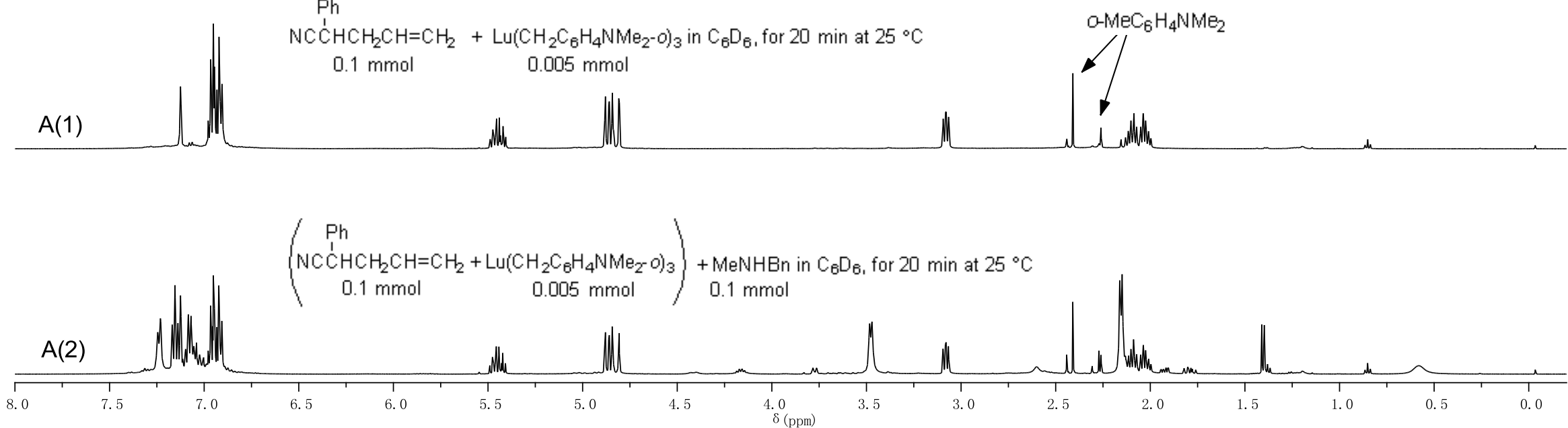


\section{Procedure B}

$\mathrm{Lu}\left(\mathrm{CH}_{2} \mathrm{C}_{6} \mathrm{H}_{4} \mathrm{NME}_{2}-\mathrm{O}\right)_{3}$ in $\mathrm{C}_{6} \mathrm{D}_{6}$

lon

MeNHBn in $\mathrm{C}_{6} \mathrm{D}_{6}$

2a

Nhun

$\| \quad \stackrel{\mathrm{Ph}}{\mathrm{N} C H C H} \mathrm{HCH}_{2} \mathrm{CH}=\mathrm{CH}_{2}$ in $\mathrm{C}_{6} \mathrm{D}_{6}$

$1 \mathrm{a}$

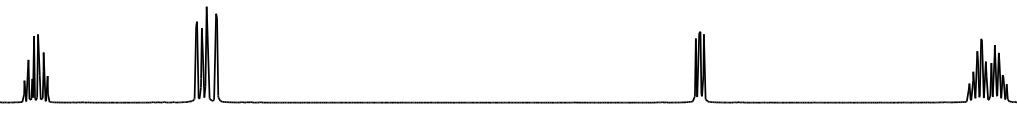

$\mathrm{Ph}$

$\mathrm{NCCHCH}{ }_{2} \mathrm{CH}=\mathrm{CH}_{2}+\mathrm{MeNHBn}$ in $\mathrm{C}_{6} \mathrm{D}_{6}$, for 20 min at $25^{\circ} \mathrm{C}$ $0.1 \mathrm{mmol}$ $0.1 \mathrm{mmol}$

B(1) Hun

Whe M M

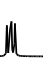

Whill

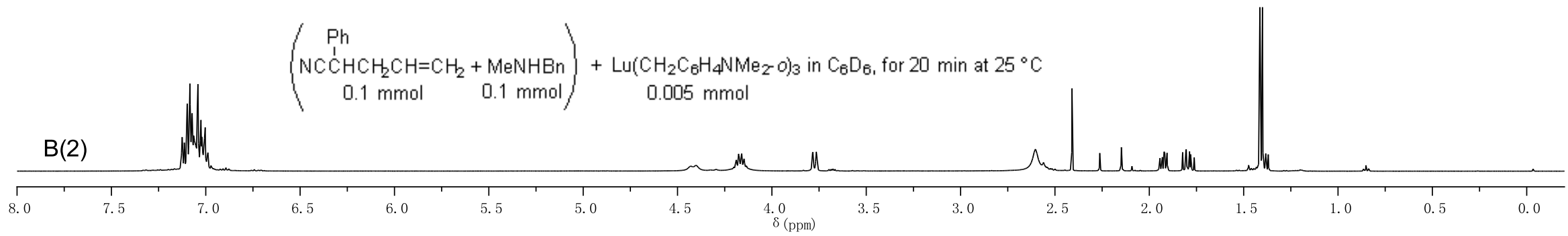




\section{Procedure C}

$\mathrm{Lu}\left(\mathrm{CH}_{2} \mathrm{C}_{6} \mathrm{H}_{4} \mathrm{NME}_{2}-\mathrm{O}\right)_{3}$ in $\mathrm{C}_{6} \mathrm{D}_{6}$

$\ln m$ an

MeNHBn in $\mathrm{C}_{6} \mathrm{D}_{6}$

2a

Nilune

$\mathrm{Ph}$

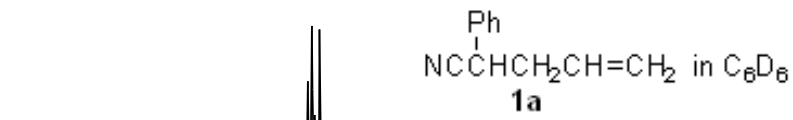

1a

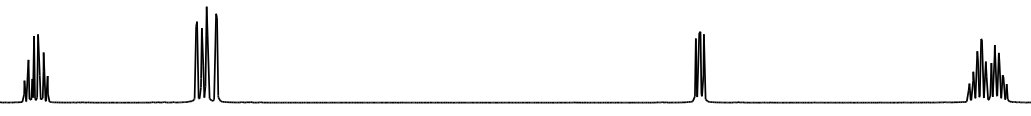

MeNHBn + $\mathrm{Lu}\left(\mathrm{CH}_{2} \mathrm{C}_{6} \mathrm{H}_{4} \mathrm{NMe}_{2}-\mathrm{o}\right)_{3}$ in $\mathrm{C}_{6} \mathrm{D}_{6}$, for 20 min at $25{ }^{\circ} \mathrm{C}$

$0.1 \mathrm{mmol} \quad 0.005 \mathrm{mmol}$

o- $\mathrm{MeC}_{6} \mathrm{H}_{4} \mathrm{NME}_{2}$
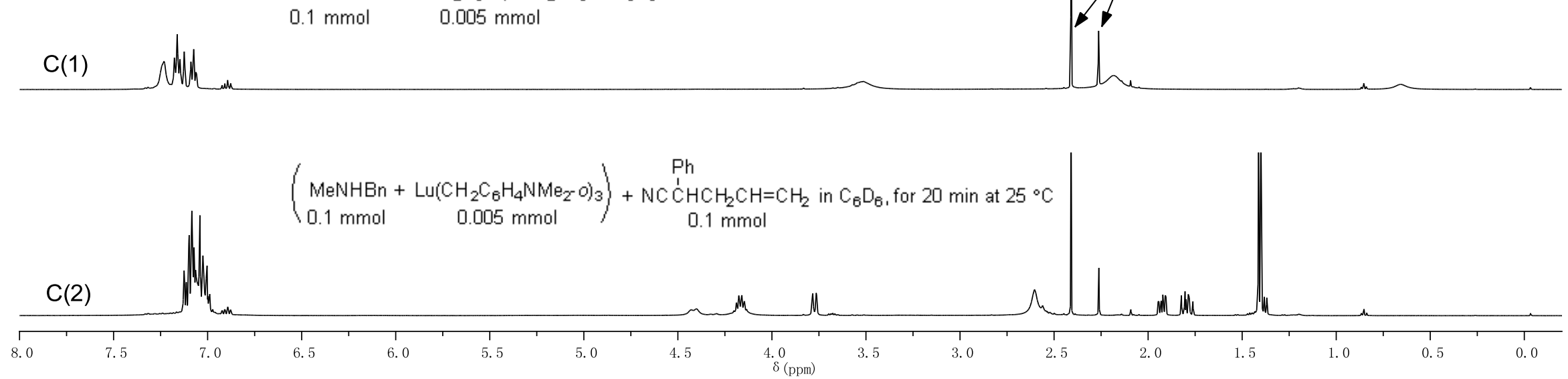
13. Temperature-Dependent ${ }^{1} \mathrm{H}$ NMR Studies of $3 \mathrm{aa}$ in $\mathrm{CDCl}_{3}$

Compound ( \pm )-3aa (purified sample after column chromatography)
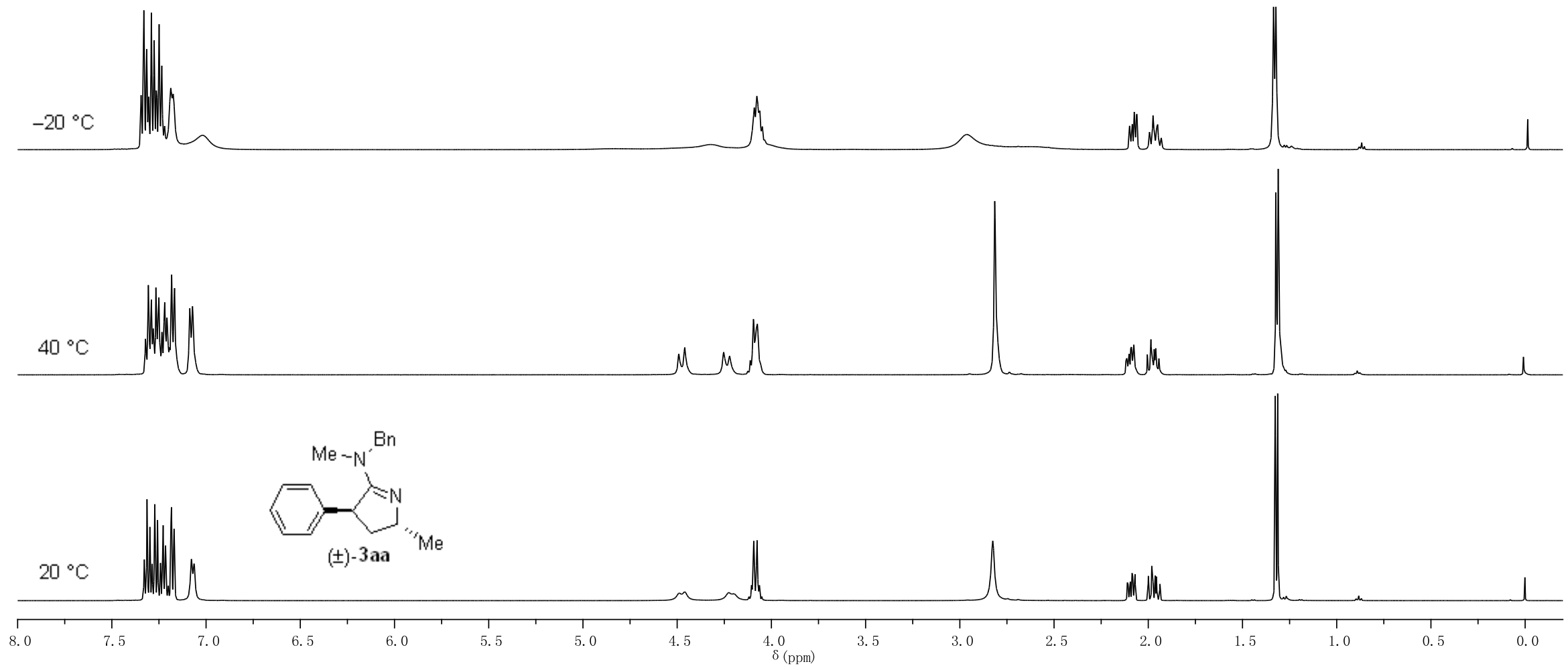
( \pm )-3aa (crude sample before column chromatography)
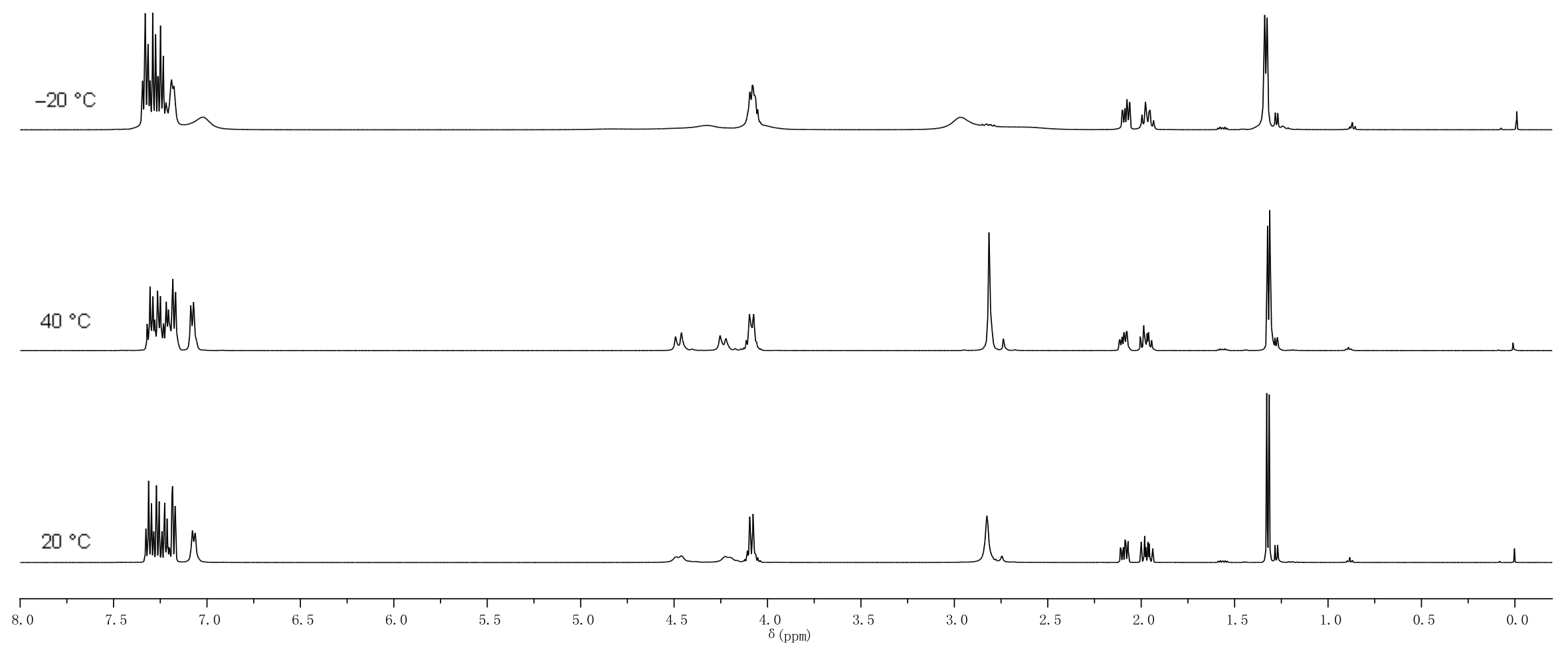